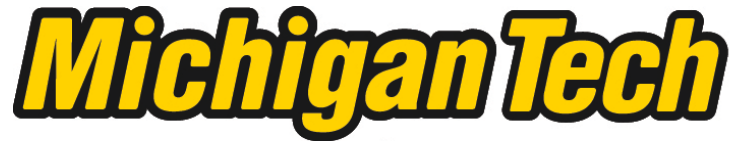 \\ Michigan Technological University Create the Future Digital Commons @ Michigan Tech
}

Dissertations, Master's Theses and Master's Reports - Open

Dissertations, Master's Theses and Master's

Reports

2008

\section{Quantum dot / optical protein bio-nano hybrid system biosensing}

Mark H. Griep

Michigan Technological University

Follow this and additional works at: https://digitalcommons.mtu.edu/etds

Part of the Mechanical Engineering Commons

Copyright 2008 Mark H. Griep

\section{Recommended Citation}

Griep, Mark H., "Quantum dot / optical protein bio-nano hybrid system biosensing", Dissertation, Michigan Technological University, 2008.

https://doi.org/10.37099/mtu.dc.etds/369

Follow this and additional works at: https://digitalcommons.mtu.edu/etds

Part of the Mechanical Engineering Commons 


\title{
QUANTUM DOT / OPTICAL PROTEIN BIO-NANO HYBRID SYSTEM BIOSENSING
}

\author{
By \\ Mark H. Griep \\ A DISSERTATION \\ Submitted in partial fulfillment of the requirements \\ for the degree of \\ DOCTOR OF PHILOSOPHY \\ (Mechanical Engineering - Engineering Mechanics) \\ MICHIGAN TECHNOLOGICAL UNIVERSITY \\ 2008
}

Copyright $@$ Mark H. Griep 2008 
This dissertation, "Quantum Dot / Optical Protein Bio-Nano Hybrid System Biosensing," is hereby approved in partial fulfillment of the requirements for the degree of DOCTOR OF PHILOSOPHY in the field of Mechanical Engineering-Engineering Mechanics.

\section{DEPARTMENT:}

Mechanical Engineering-Engineering Mechanics

Signatures:

Dissertation Advisor

Dr. Craig Friedrich

Department Chair

Dr. William Predebon

Date 


\section{$\underline{\text { ABSTRACT }}$}

The integration of novel nanomaterials with highly-functional biological molecules has advanced multiple fields including electronics, sensing, imaging, and energy harvesting. This work focuses on the creation of a new type of bio-nano hybrid substrate for military biosensing applications. Specifically it is shown that the nano-scale interactions of the optical protein bacteriorhodopsin and colloidal semiconductor quantum dots can be utilized as a generic sensing substrate.

This work spans from the basic creation of the protein to its application in a novel biosensing system. The functionality of this sensor design originates from the unique interactions between the quantum dot and bacteriorhodopsin molecule when in nanoscale proximity. A direct energy transfer relationship has been established between coreshell quantum dots and the optical protein bacteriorhodopsin that substantially enhances the protein's native photovoltaic capabilities. This energy transfer phenomena is largely distance dependent, in the sub-10nm realm, and is characterized experimentally at multiple separation distances. Experimental results on the energy transfer efficiency in this hybrid system correlate closely to theoretical predictions.

Deposition of the hybrid system with nano-scale control has allowed for the utilization of this energy transfer phenomena as a modulation point for a functional biosensor prototype. This work reveals that quantum dots have the ability to activate the bacteriorhodopsin photocycle through both photonic and non-photonic energy transfer mechanisms. By altering the energy transferred to the bacteriorhodopsin molecule from the quantum dot, the electrical output of the protein can be modulated. A biosensing prototype was created in which the energy transfer relationship is altered upon target binding, demonstrating the applicability of a quantum dot/bacteriorhodopsin hybrid system for sensor applications. The electrical nature of this sensing substrate will allow for its efficient integration into a nanoelectronics array form, potentially leading to a small-low power sensing platform for remote toxin detection applications. 


\section{DEDICATION}

This work is dedicated to my late grandfather and friend, Leo McMahon, whose great out-look on life has provided inspiration for mine. 


\section{ACKNOWLEDGEMENTS}

This doctoral work would not have been possible without the gracious guidance and support of countless individuals in my life. I would first like to thank my parents, Howard and Diane Griep, for encouraging me to continue my education and supporting me through it all.

For this Ph.D. work I have been extremely privileged to have Dr. Craig Friedrich as my advisor and mentor. Since bringing me into his group as an undergraduate, his guidance has taught me how to perform scientific research and his vision brought the novel biosensors project to MTU that I was fortunate to explore. In addition his efforts and vision for my professional development have allowed my participation in multiple external research opportunities and conferences which have substantially enhanced my education.

I would also like to thank my co-advisor, Dr. Donald Lueking, for his enthusiasm and patience during this project. His insight to the world of micro-biology and ability to pass on this knowledge in a manner understandable even to Mechanical Engineers has been critical to the success of this work. His dedication to this work, even after retirement, has been greatly appreciated and I'm sure I will seek his advice for years to come.

Upon arriving at MTU in 2002 I was fortunate to become friends with Dr. Debra Charlesworth, who has had a profound impact on my life over the past 6 years. Dr. Charlesworth taught me the importance gaining an education outside of the classroom and how to give back to the local community. Her commitment to my development brought me to where I am today.

During the course of this work I was welcomed into Dr. Karna's research group at the Army Research Lab. I would like to thank Dr. Karna for giving me the opportunity to participate in his group's research efforts and also for the countless hours of teachings on 
the fundamentals of quantum physics. Dr. Govind Mallick and Dr. Raymond Mackay were a pleasure to work with at ARL and I look forward to future collaborations. I would also like to thank the families of Dr. Karna and Dr. Mallick for making me feel at home during my stay at ARL.

Last, but not least, I would like to thank Dr. Michelle Miller and Dr. Gregory Odegard for offering their time and assistance as members of my thesis committee. My graduate student colleagues on this work, including Eric Winder, Karl Walczak, Chris Anton, Dawdon Cheam, and Tracy Curtis have also provided tremendous aid on the project and endless humor to help pass the long hours in the lab. 


\section{$\underline{\text { TABLE OF CONTENTS }}$}

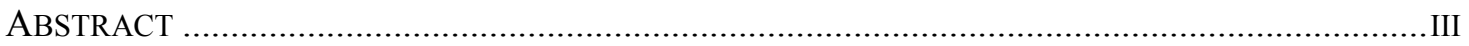

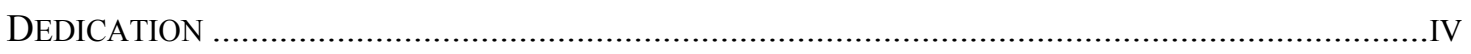

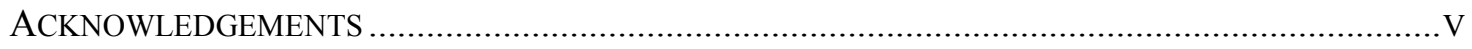

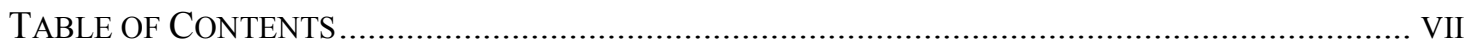

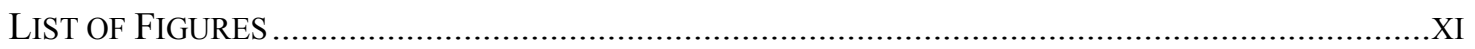

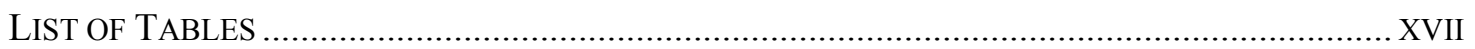

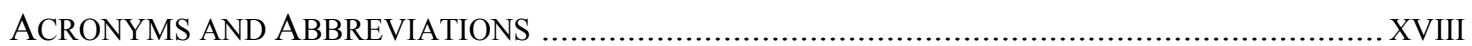

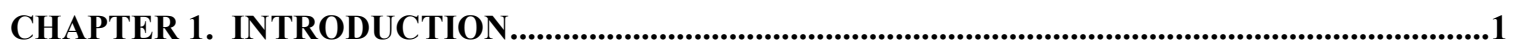

1.1. MOTIVATION

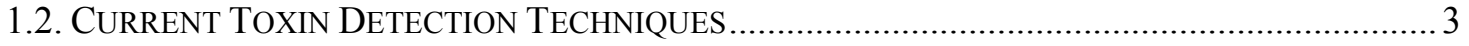

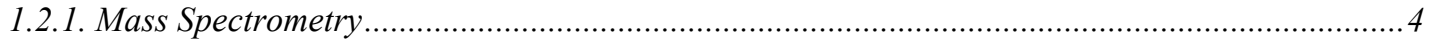

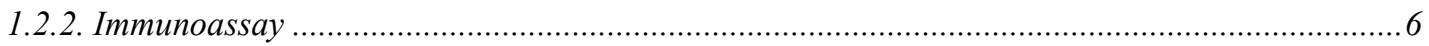

1.2.3. Laser-Based Fluorescence ….............................................................................................. 7

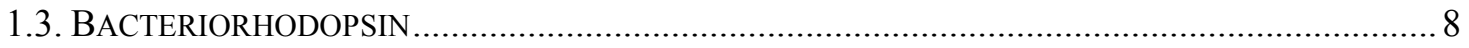

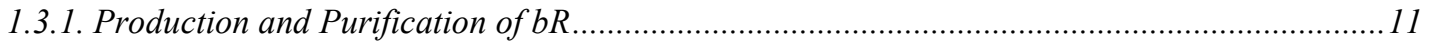

1.3.2. Applying bR to Engineered Applications .......................................................................... 12

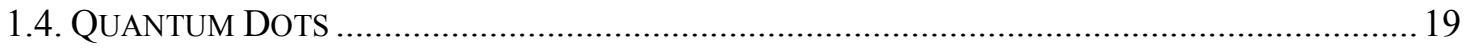

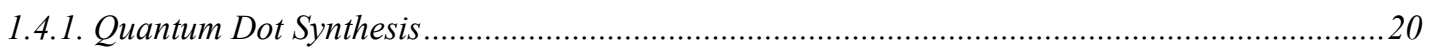

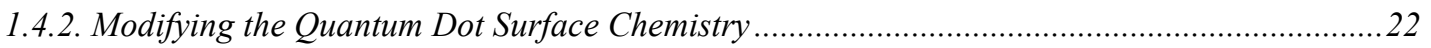

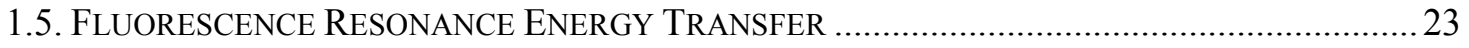

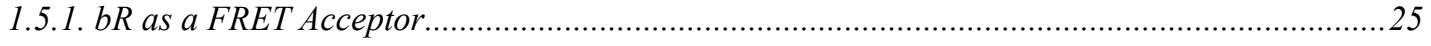

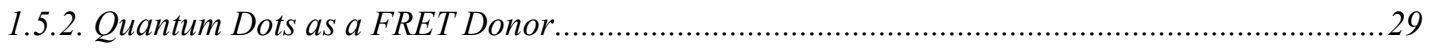

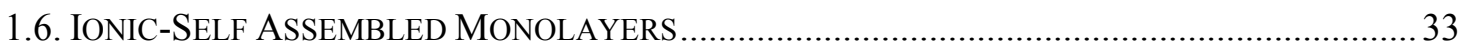

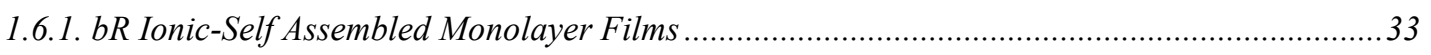




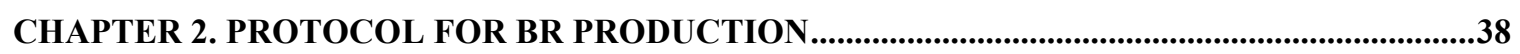

CHAPTER 3. BR-BASED ELECTRODES .................................................................................................43

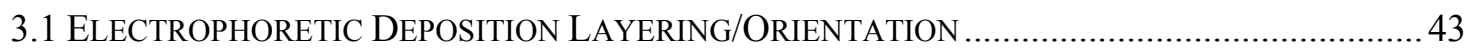

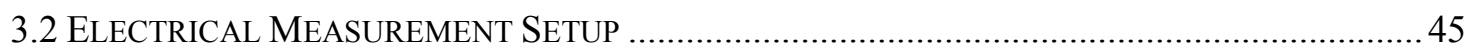

3.3 Photoelectric Activity of Electrodeposited PM Films........................................... 46

3.4 TEMPERATURE DEPENDENCE OF BR PHOtovoltaic RESPONSE....................................... 51

CHAPTER 4. QD ACTIVATION OF BR PHOTOVOLTAIC RESPONSE .........................................58

4.1 EFFECTS OF CONTINUOUS UV ILLUMination ON BR PHotovoltage .............................59

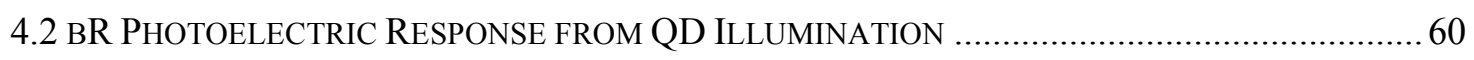

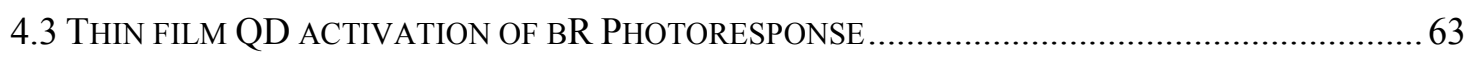

CHAPTER 5. QD-BR HYBRID IONIC-SELF ASSEMBLED MONOLAYERS ................................68

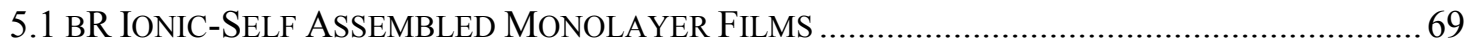

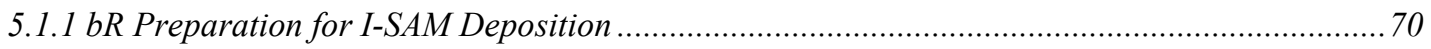

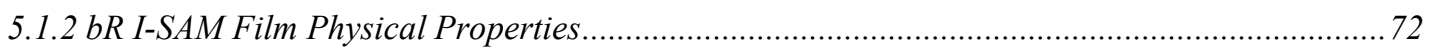

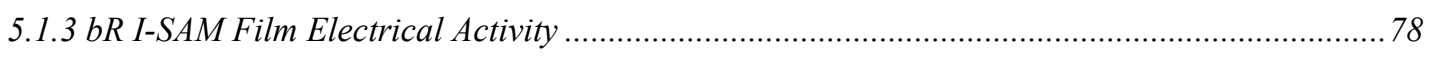

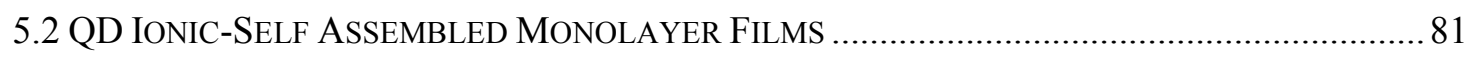

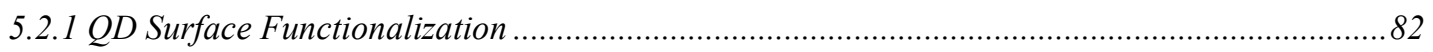

5.2.2 QD I-SAM Film Physical and Optical Properties.................................................................. 84

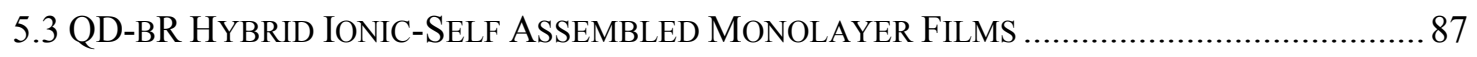

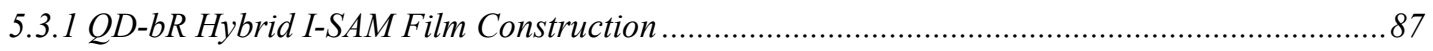

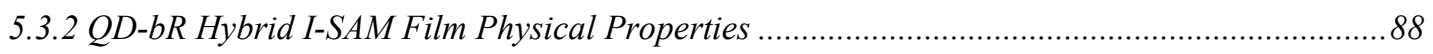

5.3.3 QD-bR Hybrid I-SAM Film Electrical Activity .................................................................... 91

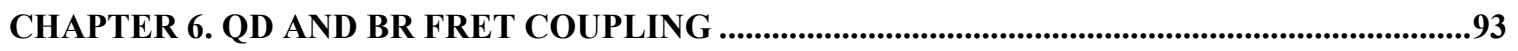

viii 


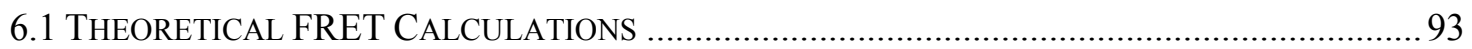

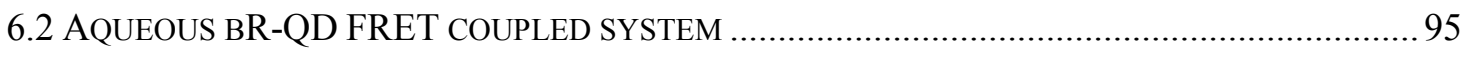

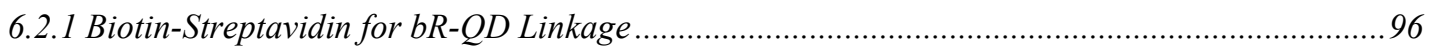

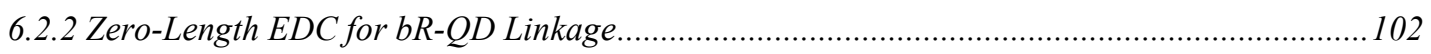

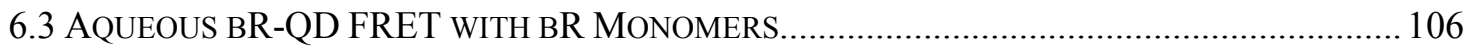

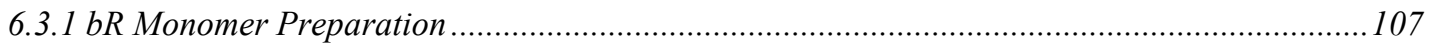

6.3.2 Biotin-Streptavidin for bR Monomer-QD Linkage ............................................................... 109

6.3.2 Zero-Length EDC for bR Monomer-QD Linkage .................................................................. 113

6.4 QD LIFETIME DECAY ANALYSIS WITH EDC-LINKED BR-QD PAIRS ............................... 119

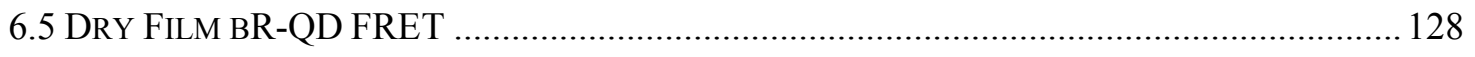

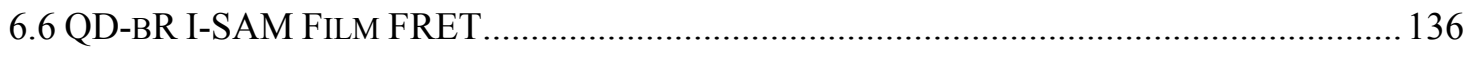

CHAPTER 7. MBP SENSOR PROOF OF CONCEPT ................................................................139

7.1 SyntheSis AND PURIFICATION OF MALtose Binding ProteIn..................................... 141

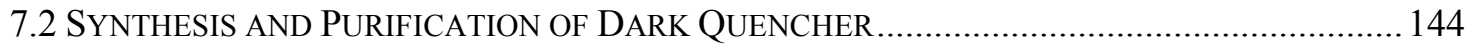

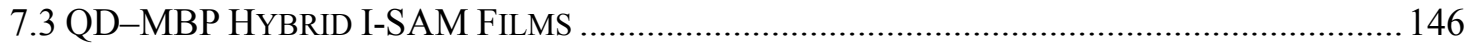

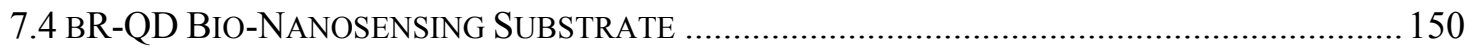

8.0 SUMMARY AND CONCLUSIONS .............................................................................................156

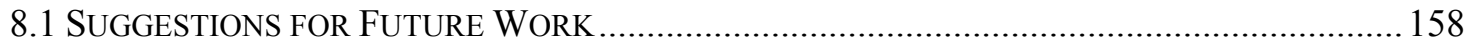

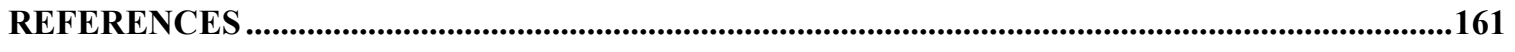

APPENDIX A: PERMISSIONS FROM PUBLISHERS ……………...........................................................169

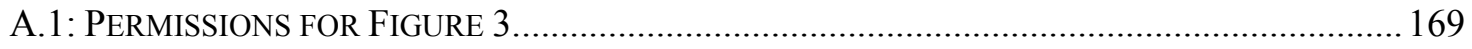

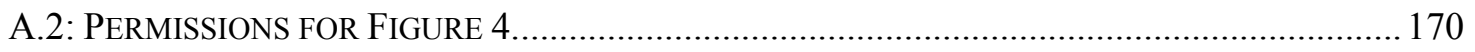

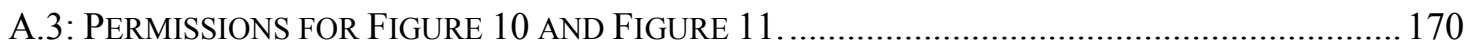

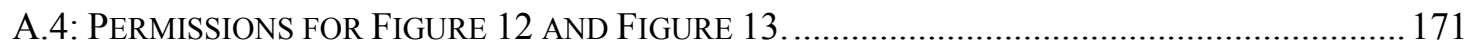

ix 
A.5: PERMisSIONS FOR Figure 18.

A.6: PERMISSIONS FOR FIGURE 19 AND FIGURE 116.

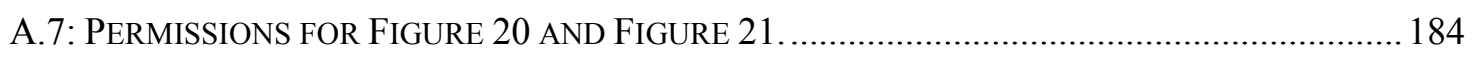

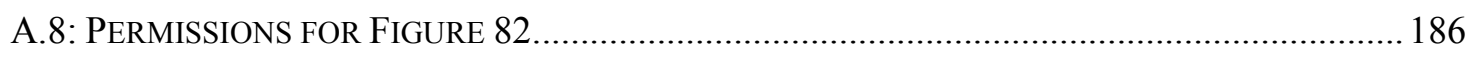




\section{LIST OF FIGURES}

Figure 1. Proposed system utilizing energy transfer mechanisms in an integrated quantum dot-bacteriorhodopsin hybrid sensor platform...................................................... 1

Figure 2. Fatal dosages to average person of select toxins ............................................. 2

Figure 3. Schematics of BAMS Pathogen Detection Unit [5]. Permission received from

Lawrence Livermore National Labs and can be found in Appendix A.1 .................. 5

Figure 4. Sandia National Lab's $\mu$ ChemLab ${ }^{\mathrm{TM}}$ [12]. Permission received from Sandia

National Labs and can be found in Appendix A.2 ………................................... 7

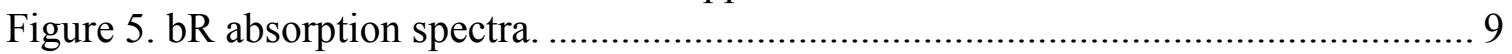

Figure 6. bR proton pumping pathway. ................................................................... 9

Figure 7. Proton-pumping cycle of $b R$, demonstrating the photocycle intermediates and

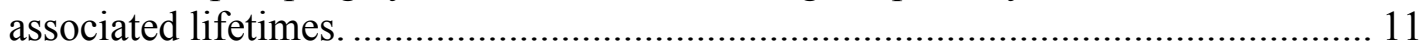

Figure 8. Charge of bR molecule at neutral pH......................................................... 13

Figure 9. Sample bR film electrical output upon light illumination [30]...................... 15

Figure 10. Schematic of FET with integrated PM film [35]. Permission received from the

Optical Society of America and can be found in Appendix A.3 ........................... 16

Figure 11. Photovoltage output of unoriented PM film integrated with GaAs FET [35].

Permission received from the Optical Society of America and can be found in

Appendix A.3 ............................................................................................... 16

Figure 12. Method to pattern PM on the micron-scale [36]. Permission received from

IOP publishing and can be found in Appendix A.4 ……………......................... 17

Figure 13. SFM image of micro-scale patterned PM (scale bar $10 \mu \mathrm{m}$ ) [36]. Permission

received from IOP publishing and can be found in Appendix A.4 ……………...... 18

Figure 14. Typical spectral emission ranges for synthesized CdS, CdSe, and CdTe core

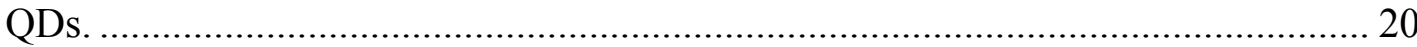

Figure 15. Blue-shift of 585nm emission CdSe/ZnS QD upon oxidation [58]. ............. 22

Figure 16. Overlap of NBD emission (solid line) and bR Retinal Absorption (dotted line)

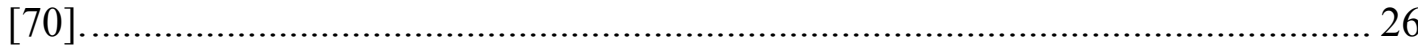

Figure 17. PY emission (curve 1), dark-adapted bovine rhodopin absorption (curve 2) and photo-bleached bovine rhodopsin absorption (curve 3) [68] ............................ 27

Figure 18. Absorption spectra of bR before and after photo-bleaching by continuous light (a) at different $\mathrm{pH}$ values $\left(50^{\circ} \mathrm{C}, 30 \mathrm{~min}\right.$ illumination at $\left.400 \mathrm{~mW} / \mathrm{cm}^{2}\right)$, (b) at different percentages of maximum illumination power of $400 \mathrm{~mW} / \mathrm{cm}^{2}\left(50^{\circ} \mathrm{C}, \mathrm{pH} 9.5,30 \mathrm{~min}\right.$ illumination), (c) at different temperatures ( $\mathrm{pH} 9.5,60 \mathrm{~min}$ illumination at $\left.400 \mathrm{~mW} / \mathrm{cm}^{2}\right)$, and (d) at different bleaching times $\left(50^{\circ} \mathrm{C}, \mathrm{pH} 9.5,400 \mathrm{~mW} / \mathrm{cm}^{2}\right.$ illumination power) [71]. Reprinted from FEBS Letters, Vol. 450, Dancshazy et al., Bleaching of Bacteriorhodopsin by Continuous Light, p. 154-157, 1999, with permission from Elsevier [OR APPLICABLE SOCIETY COPYRIGHT OWNER] and can be found in Appendix A.5. ............................................................... 28

Figure 19. Schematic of maltose displacement of $\beta$-CD-QSY9 in a MBP-5HIS/QD bioconjugate [41]. Reprinted by permission from Macmillan Publishers Ltd: Nature Materials, Medintz, I.L., et al., Self-assembled nanoscale biosensors based on 
quantum dot FRET donors, 2: p. 630-638, copyright (2003) and permissions can be found in Appendix A.6.

Figure 20. Schematic of hybridization of Au-DNA with immobilized QD-DNA-biotin

[74]. Dyadyusha, L., et al., Quenching of CdSe quantum dot emission, a new approach for biosensing. Chem. Commun., 2005: p. 3201-3203 - Reproduced by permission of The Royal Society of Chemistry. Permission can be found in Appendix A.7

Figure 21. Epiflourescence image of i) QD-DNA-biotin associated on streptavidin functionalized glass slide, ii) after addition of Au-DNA ( $5 \mathrm{~min}$ ), iii) after addition of Au-DNA (15 min) [74]. Dyadyusha, L., et al., Quenching of CdSe quantum dot emission, a new approach for biosensing. Chem. Commun., 2005: p. 3201-3203 Reproduced by permission of The Royal Society of Chemistry. Permission can be found in Appendix A.7.

Figure 22. Schematic of bilayer I-SAM films constructed on negatively charged ITO with PDAC serving as the polycation and PM serving as the polyanion................. 34

Figure 23. Photocurrent generated from multi-layered PM films produced with the I-

SAM technique. Maximal output is achieved between 6 to 8 bilayers for the two different types of PM tested [80]. 35

Figure 24. QD surface modifications for I-SAM applications. ....................................... 36

Figure 25. Outline of H. halobium production and PM isolation/purification. ............... 39

Figure 26. Sucrose gradient percentages for $b R$ purification. .......................................... 41

Figure 27. Particle size analysis of PM fragments showing an average fragment diameter of $497.6 \mathrm{~nm}$ with a size range of $399.3 \mathrm{~nm}-536.4 \mathrm{~nm}$........................................ 42

Figure 28. PM orientation thru electrophoretic sedimentation ........................................ 44

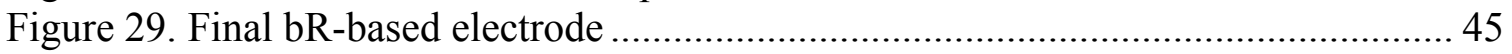

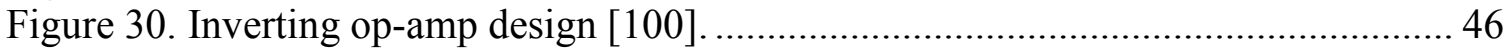

Figure 31. Photocyle intermediate of dried film bR after photo-illumination.................. 47

Figure 32. bR Photoelectric response to incadescent light illumination with AC-coupling

$(8 \mathrm{mV} / \mathrm{vertical}$ division, $1 \mathrm{sec} /$ horizontal division).................................................. 48

Figure 33. bR Photoelectric response to incadescent light illumination with DC-coupling

(20 mV/vertical division, 5sec/horizontal division)............................................... 48

Figure 34. bR photoelectric response to $\sim 8 \mathrm{~mW} / \mathrm{cm} 2$ illumination with $10 \mathrm{x}$ amplification

$(100 \mathrm{mV} /$ vertical division, $0.7 \mathrm{sec} /$ horizontal division)............................................... 49

Figure 35 . bR photoelectric response to $4 \mathrm{~Hz}$ light illumination $(8 \mathrm{mV} / \mathrm{vertical}$ division,

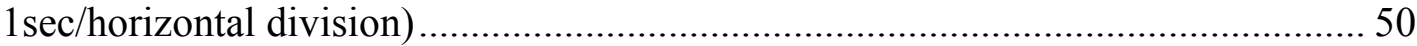

Figure 36. PM photovoltaic response at elevated temperatures [97]............................. 52

Figure 37. Effect of temperature increase on bR photovoltaic response and subsequent

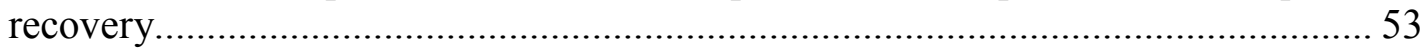

Figure 38. Waveform of bR photovoltaic response during temperature increase. ........... 54

Figure 39. Effect of decreasing temperature on bR photovoltaic response and subsequent

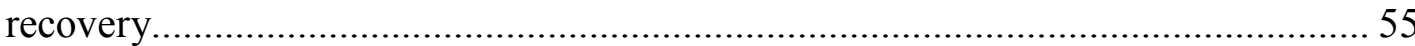

Figure 40. Alterations in bR photovoltaic waveform based on temperature. The same bR sample displays a differential photovoltaic response at $21^{\circ} \mathrm{C}\left(70^{\circ} \mathrm{F}\right)$, with the response waveform changing to steady-state voltage at sub-freezing temperatures. 57

Figure 41. 565nm QD emission spectra and bR absorption spectra overlap ................... 58 
Figure 42. bR electrode signal from $8 \mathrm{~mW} / \mathrm{cm} 2$ light illumination before UV illumination

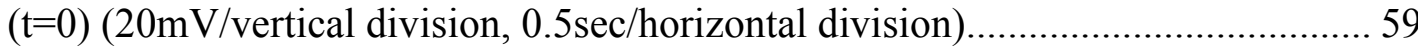

Figure 43. bR electrode signal from $8 \mathrm{~mW} / \mathrm{cm} 2$ light illumination following UV

illumination ( $\mathrm{t}=95$ minutes) $(20 \mathrm{mV} /$ vertical division, $0.5 \mathrm{sec} /$ horizontal division) .. 60

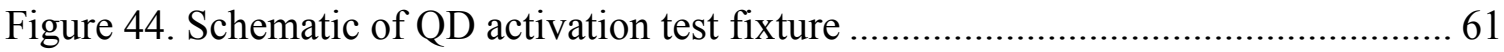

Figure 45. 310nm UV LED emission spectrum......................................................... 61

Figure 46. bR photoelectric response to 4Hz UV flashing - no QD's ( $1 \mathrm{mV} /$ vertical

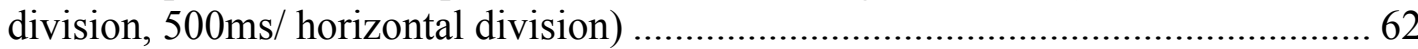

Figure 47. bR photoelectric response to $4 \mathrm{~Hz}$ QD illumination $(2 \mathrm{mv} /$ vertical division,

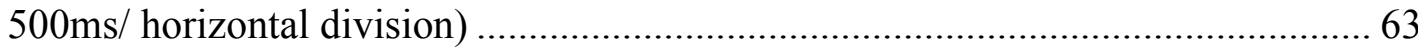

Figure 48. Emission spectra of pre and post biotinylated bR electrodes after addition of $595 \mathrm{~nm}$ streptavidin-coated QDs

Figure 49. Photovoltaic response of pre-biotinylated bR electrode with and without QD addition.

Figure 50. Schematic of QD activation of $b R$ photoresponse via photonic and FRET methods.

Figure 51. Schematic of nanoparticle proximity achieved through I-SAM deposition techniques.

Figure 52. Zeta potential analysis of PM fragments at $\mathrm{pH} 9.5$ showing an average PM

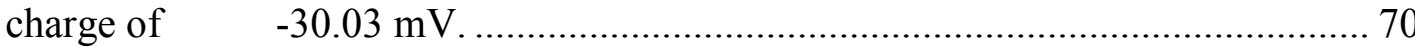

Figure 53. Materials used for I-SAM deposition. From left to right: PM, QD, PDAC. 71 Figure 54. 3-dimensional AFM topography image of blank ITO used for PM deposition.

Figure 55. AFM topography image of blank ITO slide used for PM deposition............. 73

Figure 56. 3-dimensional AFM topography image of 1 PM monolayer deposited on PDAC .74

Figure 57. AFM topography scan of of 1 PM monolayer deposited on PDAC............... 74

Figure 58. AFM height profile of 1 PM monolayer deposited on PDAC ........................ 75

Figure 59. Absorbance spectra of PDAC in $0.5 \mathrm{M} \mathrm{NaCL}$ at $\mathrm{pH} 6.8$............................ 76

Figure 60. Layering process to create $\mathrm{bR} / \mathrm{PDAC}$ multi-layered monolayer films........... 76

Figure 61. bR absorbance spectra for select bR/PDAC bilayers as it is assembled.

Bilayers constructed on negatively charded ITO with PDAC (+) and bR (-) being

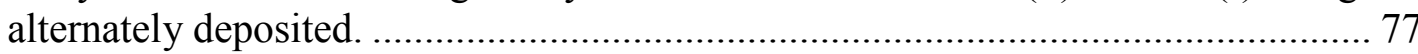

Figure 62 . The $\mathrm{bR}_{570} \mathrm{~nm}$ absorption peaks for consecutively deposited bR/PDAC bi-

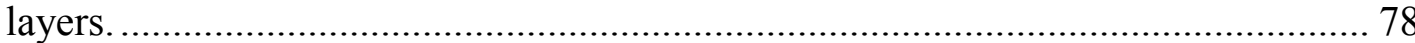

Figure 63. Photovoltaic response of a 12 monolayer bR I-SAM film (10mW halogen light source) 79

Figure 64. Photocurrent response of a 12 monolayer bR I-SAM film (10mW halogen

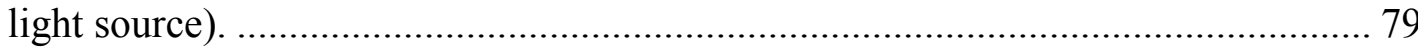

Figure 65. Comparison of 3 monolayer and 6 monolayer PM I-SAM film photovoltaic output. 80

Figure 66. Effect of varying light intensity on 3 monolayer PM photovoltaic output..... 81 Figure 67. Emission and absorbance spectra of TGA-capped CdSe/ZnS quantum dots.. 82 Figure 68. Layering process to create QD/PDAC multi-layered monolayer films.......... 83

Figure 69. QD emission tracking QD/PDAC bilayer deposition.................................... 84 Figure 70. $\mathrm{QD}_{568 \mathrm{~nm}}$ emission maximum tracked through mulit-layered QD deposition. . 85 
Figure 71. QD emission comparison when deposited on differently charged substrates. 86 Figure 72. Layering process to create QD/PDAC/bR multi-layered monolayer films..... 88 Figure 73. QD emission tracked during QD (solid lines)/PDAC/bR (dotted lines) multilayered films.....

Figure 74. QD $_{568 \mathrm{~nm}}$ emission maxima tracked during each QD-bR monolayer deposition.

Figure 75. AC-coupled output of a 3 monolayer bR film (1mv/div, $1 \mathrm{sec} / \mathrm{div})$.............. 92 Figure 76. AC-coupled output of 3 monolayer bR film topped with 1 monolayer QD

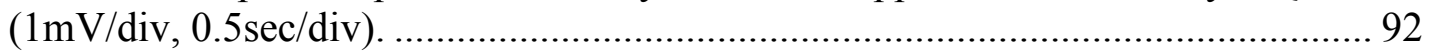

Figure 77. Theoretical FRET efficiency of a 565nm QD-bR coupled system. ............... 95

Figure 78. Schematic of bR-QD linkage via a biotin-streptavidin binding scheme........ 97

Figure 79. HABA/avidin assay for quantifying biotinylation of $b R$ molecules. Blue squares are control baseline and red squares are the biotinylated bR samples........ 99

Figure 80. bR-QD FRET coupling via biotin-streptavidin linkage. 100

Figure 81. Tracking of normalized 595nm streptavidin QD emission as ratio of bound biotinylated $\mathrm{bR}$ is increased. 101

Figure 82. Schematic of EDC cross-linking mechanism (image courtesy of Pierce

Technologies). Permission received from the Thermo Fisher Scientific and can be

found in Appendix A.8. 103

Figure 83. Schematic of bR-QD linkage via an EDC binding scheme. 103

Figure 84. QD quenching effects of the bR retinal ( $b R$ in PM patch form) when linked to

a $\mathrm{CdSe} / \mathrm{ZnS} \mathrm{QD}$ via EDC cross-linker.

Figure 85. Time progression of QD quenching during EDC linkage with bR (PM patch form).

Figure 86. bR solubilzation vs. OG concentration over 24 hour duration..................... 107

Figure 87. Degree of bR denaturation vs OG concentration over 24 hour duration....... 108 Figure 88. bR solubilization \% vs. 70mM OG pH over 24 hour duration...................... 108 Figure 89. Degree of bR denaturation vs. 70mM OG pH over 24 hour duration........... 109 Figure 90. FRET quenching of various QD:bR monomer ratios, linked via biotinstreptavidin

Figure 91. Tracking of normalized 595nm streptavidin QD emission as ratio of bound biotinylated $\mathrm{bR}$ is increased.

Figure 92. Comparison of QD quenching phenomena when linked via a biotinstreptavidin linkage to bR in both the PM fragment (red square) and bR monomer (blue diamond) forms.

Figure 93. Progression of QD quenching during EDC linkage to bR monomers.

Maximum FRET Efficiency of a coupled QD/bR monomer system is $88.2 \% \ldots \ldots .113$

Figure 94. Time progression of QD quenching via EDC linkage reaction with bR monomers

Figure 95. Comparison of QD quenching effects from EDC linked PM fragments and $b R$ monomers. Maximum FRET Efficiency of a coupled QD/PM fragment system is 49.1\% while a QD/bR monomer system yield 88.2\% FRET efficiency. Inset displays FRET coupling vs. EDC linkage reaction time.

Figure 96. Comparison of EDC and Biotin-Streptavidin linkages of QD to bR, in both $\mathrm{PM}$ patch and monomer form. 
Figure 97. Jablonski diagram showing FRET between a donor and an acceptor molecule. The purple arrow shows QD absorption, yellow arrow shows vibrational relaxation, red solid arrow shows fluorescence. Blue arrow shows non-radiative energy transfer from the donor QD to acceptor biomolecule. 120

Figure 98. QD excited state lifetime decay of carboxyl QD control with intensity in logscale.....

Figure 99. Excited state lifetime decay of QD control. Dashed lines mark the 1/e intensity location.

Figure 100. QD excited state lifetime decay of an EDC linked PM-QD system with

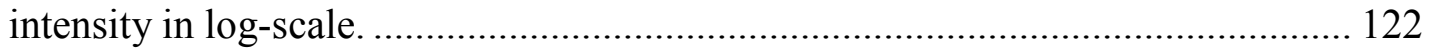

Figure 101. Excited state lifetime decay of EDC linked QD-bR (PM patch form). Dashed lines mark the $1 / \mathrm{e}$ intensity location.

Figure 102. Excited state lifetime decay of 565nm QD emission in a QD-bR monomer EDC linked system. 123

Figure 103. QD excited state lifetime of a QD-bR monomer EDC linked system plotted on a linear scale.

gure 104. Overall QD excited state lifetime comparison, with the QD control (yellow), QD-PM patch (light blue), and QD-bR monomer (green) pairs linked with EDC. 126 Figure 105. QD lifetime decay when linked to $b R$ in PM patch form $(\tau=11.58 \mathrm{~ns})$ and $b R$ monomer form ( $\tau=5.61 \mathrm{~ns})$ compared to the QD only control ( $\tau=13.93 \mathrm{~ns})$. Inset displays identical QD excited state lifetime decay data with intensity (counts) in Log-scale.

Figure 106. Fluorescence emission image of dried QD layers on top of bR film and glass

Figure 107. QD emission vs. wavelength on varying substrates

Figure 108. Timescan of 570nm QD emission vs. time on varying substrates ............. 132 Figure 109. Control studying showing QD quenching effects of $\mathrm{bR}$ and $\mathrm{bO}$ (bleached bR).

Figure 110. Reduction in CdSe and CdSe/ZnS Quantum Dot emission due to energy transfer to $b R$ in dried thin films

Figure 111. I-SAM bR/QD film suggesting that the first bR layer is FRET coupled to the QDs while subsequent bR layers only have photonic absorption quenching effects.

Figure 112. Quenching of QD emission in bR/QD I-SAM film, with a 37\% emission reduction from the first $b R$ layer and $<10 \%$ reduction for each subsequent $b R$ layer.

Figure 113. Ribbon diagram of Maltose Binding Protein......................................... 139

Figure 114. Proposed maltose sensor schematic....................................................... 140

Figure 115. Effect of incident light intensity variation on bR photovoltage. ................ 141

Figure 116. Diagram of $\beta-C D-Q S Y 9$ conjugate molecule for binding with MBP [41]. Reprinted by permission from Macmillan Publishers Ltd: Nature Materials, Medintz, I.L., et al., Self-assembled nanoscale biosensors based on quantum dot FRET donors, 2: p. 630-638, copyright (2003) and permissions can be found in Appendix A.6 
Figure 117. Nanodrop absorbance spectra of as-synthesized $\beta$-CD-QSY-9 dark quencher.

Figure 118. Schematic of QD-MBP thin film biosensor for maltose sensing. ............. 148

Figure 119. QD emission during maltose detection with a QD-MBP I-SAM film....... 149

Figure 120. bR-QD hybrid substrate functionalized with a MBP receptor for the detection of maltose

Figure 121. Photovoltaic response of 1 monolayer bR and 1 monolayer QD substrate. 152

Figure 122. Photovoltaic response of bR-QD substrate with 1 monolayer MBP deposited on top.

Figure 123. Photovoltaic response of bR-QD substrate with dark quencher bound to MBP

Figure 124. Photovoltaic response of bR-QD substrate of 3mM maltose addition to displace dark quencher. 


\section{LIST OF TABLES}

Table 1. Materials for H. halobium media. Chemicals in order of addition. .................. 40

Table 2. Amount of biotins bound to bR based on HABA/avidin assay.

Table 3. FRET efficiency biotin-streptavidin linked bR-QD system at different molar ratios

Table 4. FRET efficiency for varying QD:bR monomer ratios .................................. 111

Table 5. Comparison of theoretical FRET efficiency of $565 \mathrm{~nm}$ and $595 \mathrm{~nm}$ emission QDs linked to $b R$

Table 6. Comparison of experimental bR-QD FRET efficiency based on separation distance.

Table 7. Peak emission and QD quenching levels vs. substrate.....

Table 8. Magnitude of bR bleaching with hydroxylamine and light illumination over time.

Table 9. M9 media preparation ingredients.

Table 10. $\beta$-CD and QSY-9 molar comparisons for conjugate synthesis.

Table 11. Peak photovoltaic response of bR-QD substrate at each sensing step 154 


\section{ACRONYMS AND ABBREVIATIONS}

\begin{tabular}{|c|c|c|}
\hline$\beta-C D$ & - & $\beta$-cyclodextrin-Acceptor Dyes \\
\hline AFM & - & Atomic Force Microscopy \\
\hline ARL & - & Army Research Laboratory \\
\hline $\mathrm{Ag}$ & - & Silver \\
\hline $\mathrm{Au}$ & - & Gold \\
\hline $\mathrm{bO}$ & - & Bacterio-opsin \\
\hline bR & - & Bacteriorhodopsin \\
\hline BSA & - & Bovine Serum Albumin \\
\hline $\mathrm{CdSe}$ & - & Cadmium Selenide \\
\hline $\mathrm{CMC}$ & - & Critical Micelle Concentration \\
\hline ddI & - & Distilled Deionized Water \\
\hline DHP & - & 1,6-diphenyl-1,3,5-hexatriene \\
\hline DNA & - & Deoxyribonucleic Acid \\
\hline DHLA & - & Dihydrolipoic Acid \\
\hline EDC & - & 1-ethyl-3-(3-dimethylaminopropyl) carbodiimide hydrochloride \\
\hline EDTA & - & Ethylenediaminetetraacetic acid \\
\hline FET & - & Field-Effect Transistor \\
\hline FRET & - & Fluorescence Resonance Energy Transfer \\
\hline HABA & - & 4-Hydroxyazobenzene-2 carboxylic acid \\
\hline HEPES & - & 4-(2-hydroxyethyl)-1-piperazineethanesulfonic acid \\
\hline I-SAM & - & Ionic Self Assembled Monolayers \\
\hline IOP & - & Institute of Physics \\
\hline ITO & - & Indium Tin Oxide \\
\hline LB & - & Langmuire-Blodgett \\
\hline LBL & - & Layer by Layer \\
\hline LCt50 & - & Lethal Concentration-Time Product \\
\hline LED & - & Light Emitting Diode \\
\hline $\mathrm{m} / \mathrm{z}$ & - & Mass to Charge Ratio \\
\hline MAA & - & Mercaptoacetic Acid \\
\hline MPB & - & Maltose Binding Protein \\
\hline MS & - & Mass Spectrometry \\
\hline NBD & - & 7-nitrobenz-2-oxa-1,3-diazol-4-yl \\
\hline OG & - & Octyl-Glucoside \\
\hline OPA & - & Optical Parametric Amplifier \\
\hline QD & - & Quantum Dot \\
\hline PAA & - & Poly (allylamine) \\
\hline PSS & - & Poly (styrene sulfonate) \\
\hline PVSA & - & Poly (vinylsulfonic acid) \\
\hline PM & - & Purple Membrane \\
\hline PY & - & Pyrene \\
\hline SDS-PAGE & - & Sodium Dodecly Sulfate-PolyAcrylamide Gel Electrophoresis \\
\hline SEM & - & Scanning Electron Microscopy \\
\hline
\end{tabular}




$\begin{array}{lll}\text { SFM } & - & \text { Scanning Force Microscopy } \\ \text { Sulfo-NHS } & - & \text { N-hydroxylsulfosuccinimide } \\ \text { TB } & - & \text { Terrific Broth } \\ \text { TCSPC } & - & \text { Time Correlated Single Photon Counting } \\ \text { TEM } & - & \text { Transmission Electron Microscopy } \\ \text { TGA } & - & \text { Thioglycolic Acid } \\ \text { TOF } & - & \text { Time-of-Flight } \\ \text { TOP } & - & \text { Trioctylphosphine } \\ \text { TOPO } & - & \text { Trioctylphosphine Oxide } \\ \text { UV } & - & \text { Ultraviolet } \\ \text { ZnS } & - & \text { Zinc Sulfide }\end{array}$




\section{Chapter 1. Introduction}

The goal of this project is to develop the necessary techniques to integrate the optical protein bacteriorhodopsin with colloidal semiconductor quantum dots to create an energy coupled hybrid pair. As displayed in Figure 1, one potential application of a bacteriorhodopsin and quantum dot hybrid system lies in the field of biosensors.

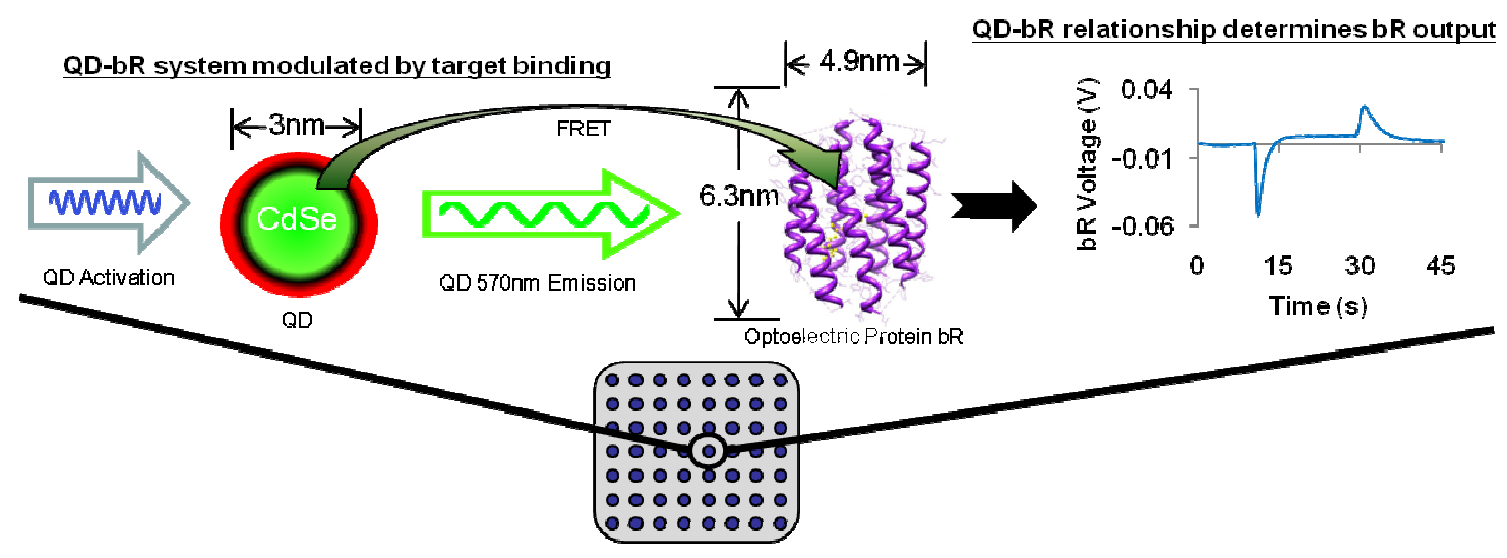

Figure 1. Proposed system utilizing energy transfer mechanisms in an integrated quantum dotbacteriorhodopsin hybrid sensor platform.

The proposed system will rely on a thorough understanding of the bio-nano interactions between bacteriorhodopsin and quantum dots. The interactions of interest include the ability of quantum dots to activate the bacteriorhodopsin photoresponse through photonic and non-photonic energy transfer means, in addition to elucidating this phenomenon in nano-scale films. Ultimately an understanding of bacteriorhodopsin and quantum dot energy coupling relationships will provide an extremely sensitive modulation point to alter bacteriorhodopsin photovoltaic output upon target molecule binding. The culmination of this work will provide a multi-functional bio-nano substrate for potential biosensing applications. 


\subsection{MotiVATION}

The need for the U.S. military to defend itself against bio-warfare attacks dates back to the use of smallpox by the British during the Revolutionary War. Since that time, the science behind bio-warfare has advanced dramatically, providing a wide array of lethal agents and efficient delivery vehicles that could be used against U.S. troops and the civilian population [1]. After nearly 100,000 casualties and millions sickened by poisonous gas in WWI, the inhumane nature of such weapons was realized and the Geneva Convention implemented a ban on such weapons in warfare.

Since that time efforts have been ongoing to ban bio and toxin based weapons all together, ultimately culminating in an international treaty that signed by nearly 150 countries in 1975. This treaty declared an end to all development, production, and stockpiling of bio and toxin weapons by virtually all developed countries. Unfortunately recent history has suggested that this prohibition doesn't completely eliminate the threat of bioweapons. With the ease of knowledge access and advancement in scientific techniques, it has been shown possible for small rogue groups or even individuals to create and use biological weapons. A select group of these toxins that could be used in a bioterrorist attack is shown in Figure 2.

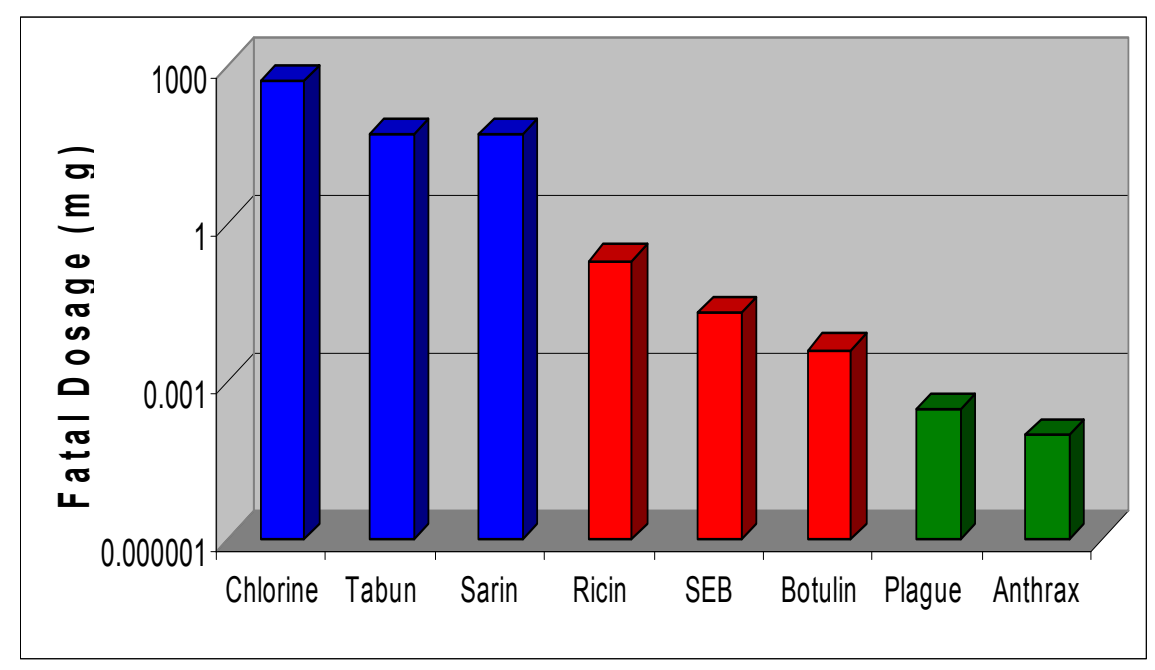

Figure 2. Fatal dosages to average person of select toxins 
With the wide array of potential agents that could be used against U.S. civilians or military personnel, it is still of vital importance to have detection systems in place. It can be noted from Figure 2, that the inhalation of only a small amount of these toxins is necessary to prove lethal to an average person. In the case of sarin gas, for example, the lethal concentration-time product (LCt50) is approximately $75 \mathrm{mg}-\mathrm{min} / \mathrm{m}^{3}$ when present in air [2]. Implying that at sarin gas concentrations of $75 \mathrm{mg} / \mathrm{m}^{3}$, an average person has a $50 \%$ chance of surviving 1 minute. Certain toxins such as sarin gas also have a cumulative effect, meaning that an individual can breathe a much lower concentration over the course of weeks to months and still reach the fatal dosage limit. Therefore the ability of a detection system to detect toxin concentrations well below their LCt50 is a necessity. Apart from the low detection limits, an efficient toxin detector will also require a rapid response time. In a biological attack one of the critical factors relating to casualty count is the time frame in which infections occur. The speed at which the toxin detector is able to identify the presence of the airborne agent therefore needs to be maximized.

From a military point of view, the ease and efficiency of device implementation is important along with the aforementioned parameters. Ideally the toxin detection system should be capable of being easily integrated with the individual soldier, creating a sensing array across the battlefield. Methods to effectively deploy an unmanned sensing apparatus into a remote/hostile area would also prove beneficial to soldier and civilian security.

\subsection{CURRENT ToXin DeTECTION TECHNiQUeS}

Science and engineering has provided a multitude of detection techniques capable of sensing a wide array of toxic agents. Many of these detection techniques can take hours or even days to perform the analysis, while others do not provide low detection thresholds. Some of the most advanced detection technologies include mass spectrometry (MS), immunoassay, and laser based fluorescence. 


\section{$\underline{\text { 1.2.1. Mass Spectrometry }}$}

For the MS detection technique, the fundamental operations are to ionize the sample, sort the ions in time or space according to their respective mass-to-charge (m/z) ratio, and finally collect/count the number of ions collected at each $\mathrm{m} / \mathrm{z}$ ratio. Typically the charge (z) will be 1, so the output spectrum can be interpreted in terms of counts vs. molecular weight.

One technique of MS in particular that is used to detect biological agents is known as single-particle aerosol MS [3, 4]. A group at Lawrence Livermore National Labs (LLNL) is developing a biological agent detection system known as bioaerosol mass spectrometry (BAMS) around this technique. As illustrated in Figure 3, a bacterial spore enters the BAMS unit and is accelerated to its terminal velocity by vacuum [5]. As the bioaerosol passes through the scattering lasers, the sizes of the individual particles and their respective velocities are measured. When the particles reach the MS chamber, they are desorbed and ionized using a $0.5 \mathrm{~J} / \mathrm{cm}^{2}$ laser pulse [6]. Using time-of-flight [7] MS, the mass of each ion is determined by measuring the time it takes for it to travel to the detector for a given electric field acceleration. The count vs. $\mathrm{m} / \mathrm{z}$ ratio plot is then constructed and pattern recognition algorithms are used to determine which airborne pathogens are present. 


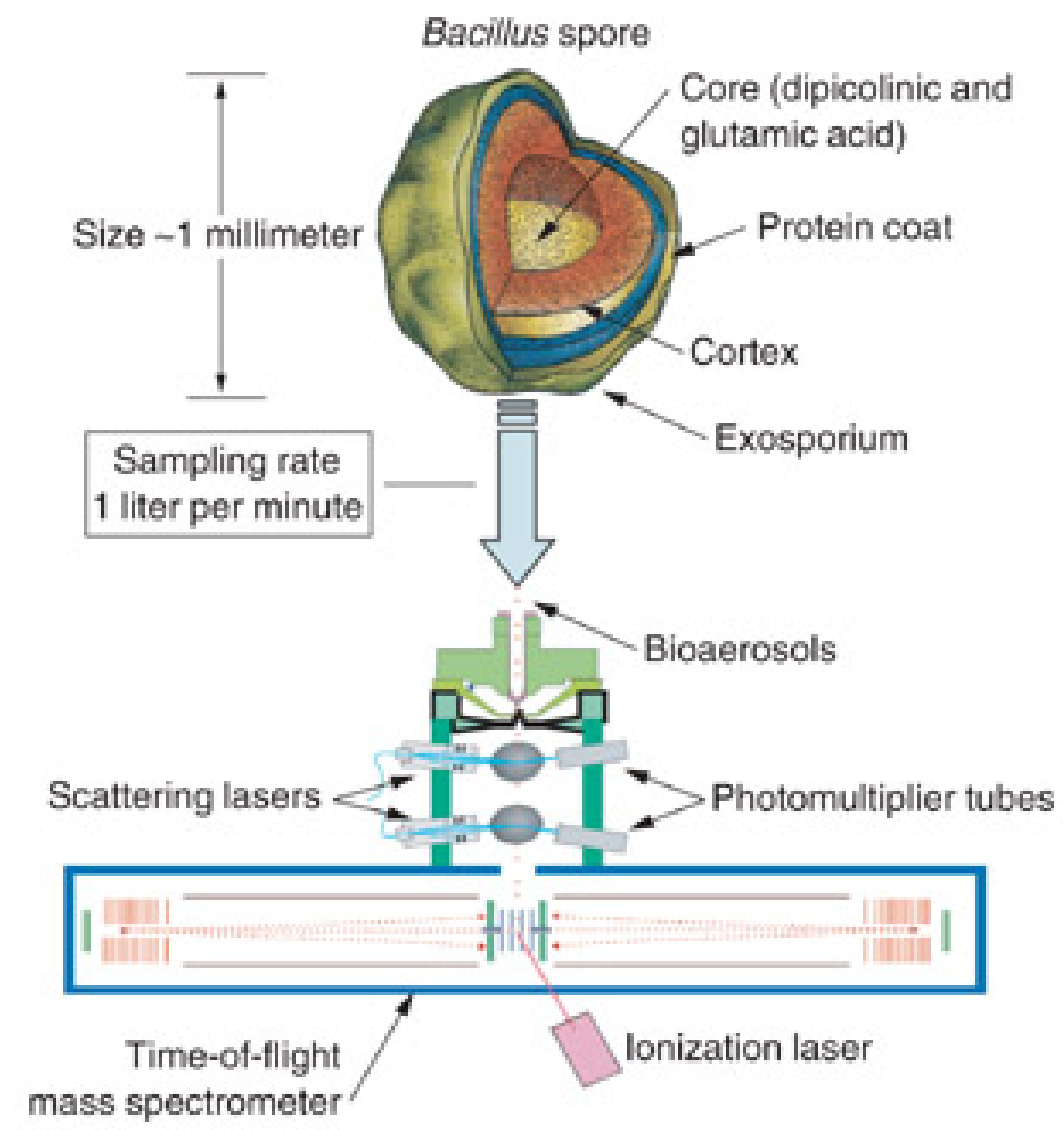

Figure 3. Schematics of BAMS Pathogen Detection Unit [5]. Permission received from Lawrence Livermore National Labs and can be found in Appendix A.1.

The BAMS unit has the capability of detecting Mycobacterium tuberculosis, the causative agent of tuberculosis, at concentrations $>40 \mathrm{M}$ in under 1 minute in a background free environment [6]. When simultaneously analyzed with similar bacterial agents, BAMS produced a positive identification rate of M. tuberculosis of $\sim 71 \%$. As it only takes a small number of tuberculosis bacilli to infect an individual [8], the detection limit of this system will need to be reduced significantly to prove effective in the detection of Mycobacterium tuberculosisi. A BAMS prototype was also used to analyze suspicious powders for the presence of anthrax after the anthrax exposures in the U.S. Postal Service in 2001 [5]. 
Current literature for BAMS suggests that it will be used primarily for the detection of biological agents in stationary structures. There is also no current literature suggesting its ability to detect other airborne agents, such as neurotoxins. Although MS techniques have shown a high sensitivity and a quick response time, their relatively large size makes them impractical for use in easily mobile applications.

\subsubsection{Immunoassay}

Immunoassays are a common biochemical technique used to analyze the amount of a particular substance in a sample. This technique utilizes the high affinity of antigenantibody reactions for the binding of select targets. Several techniques are available, including fluorescent labeling of the antigen or antibody, to quantify the amount of the target molecule that is present.

BioWarn LLC is developing a detection technology closely related to immunoassays known as SmartSense ${ }^{\mathrm{TM}}$. The basis behind the SmartSense ${ }^{\mathrm{TM}}$ technology is a ligand binding system that produces a unique electrical output signal upon binding of the target molecule [9]. The term 'ligand' is a broad term encompassing any atom, ion, or molecule, including antigens, which will bind with a high affinity to a sensor molecule. The process for target molecule identification with the SmartSense system utilizes a sensor molecule/ligand binding system. Initially the target-specific sensor molecules are oriented on top of the detector, which is the gate of a field effect transistor (FET) [10]. When a ligand binds to its specific sensor molecule, presumably a unique, measurable, electrical signal is produced by the system. The electrical output of the binding event is compared with an onboard library of electrical signatures to confirm the presence of the target molecule.

This technique has been shown to detect the presence of pathogens, such as the H5N1 virus, as described in US Patent \#7075426 [10]. A target application for this technology 
is to prevent the spread of nosocomial infections in hospitals using SmartSand ${ }^{\mathrm{TM}}$, which is envisioned to be a millimeter sized version of SmartSense ${ }^{\mathrm{TM}}$ [11].

\subsubsection{Laser-Based Fluorescence}

Laser-based fluorescence is a technique where target molecules are specifically tagged with a fluorescent molecule or dye. The laser then flashes the sample and the fluorescent molecules, whose excitation peak is close to the laser emission wavelength, begin to fluoresce at a longer wavelength than the laser emission. The emission from the fluorescent molecules is monitored and the amount of the target molecule present in the sample can be calculated.

The Sandia National Laboratory is developing a system called the $\mu$ ChemLab $^{\mathrm{TM}}$ Bio Detection System based primarily on laser-based fluorescence technology. The $\mu \mathrm{ChemLab}{ }^{\mathrm{TM}}$ is a handheld device, as shown in Figure 4, that has already been shown to accurately detect agents such as Bacillus anthracis and Clostridium botulinum neurotoxins [12].

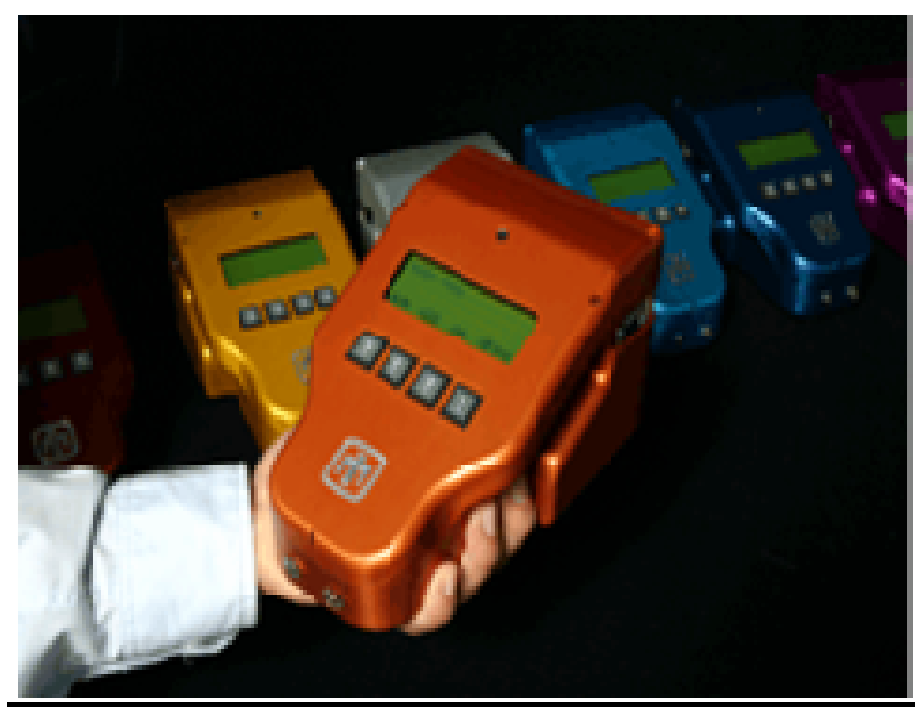

Figure 4. Sandia National Lab's $\mu$ ChemLab ${ }^{\text {TM }}$ [12]. Permission received from Sandia National Labs and can be found in Appendix A.2. 
The system works by initially loading the instrument with a liquid sample, which has already been incubated with a fluorescent dye to selectively label any target molecules [12]. Any bacterial cells are lysed through heat, pressure, and chemical degradation techniques. The proteins within the bacteria are solubilized through the use of a detergent. The particles in the solution are then separated based upon their molecular weight and $\mathrm{m} / \mathrm{z}$ ratio through the use of capillary gel electrophoresis and capillary zone electrophoresis, respectively, to remove any unwanted material such as unbound fluorescent dye. A laser is then used to illuminate the sample and induce fluorescence in the dyes. Detectors within the system detect fluorescence emission at this point and can calculate the concentration of the target molecule within the system. The entire process has been reported to take less than 10 minutes with a detection limit down to $1 \mathrm{pM}$.

\subsection{BACTERIORHODOPSIN}

Bacteriorhodopsin is a retinal protein that has been intensely studied over the years due to its inherent ability to function as a light-driven proton pump. It is composed of a 248 amino acid peptide with a molecular weight of $26,784 \mathrm{Da}$ [13] and possesses a covalently attached retinal group. Structurally similar to the visual rhodopsin found in the mammalian eye, bR is found in the cell membrane of Halobacterium halobium (also referred to as H. salinarium) and is utilized for ATP formation and ATP synthesis under anaerobic conditions. Bacteriorhodopsin strongly absorbs light in the region of $570 \mathrm{~nm}$, as shown in Figure 5, which closely corresponds to the visible light emission wavelength of the sun.

While the strong UV absorption of the bR molecule is due to aromatic amino acids present in the protein, such as tryptophan and tyrosine, the absorption properties in the $570 \mathrm{~nm}$ region is due to the attached retinal chromophore (vitamin A). With the energy absorbed by the retinal, a proton pumping mechanism is powered and the transport of a proton from the cytoplasmic side to the extracellular side of the cell membrane is 
achieved [14], as is shown in Figure 6. This pumping action creates a proton gradient across the cell membrane which facilitates ATP synthesis.

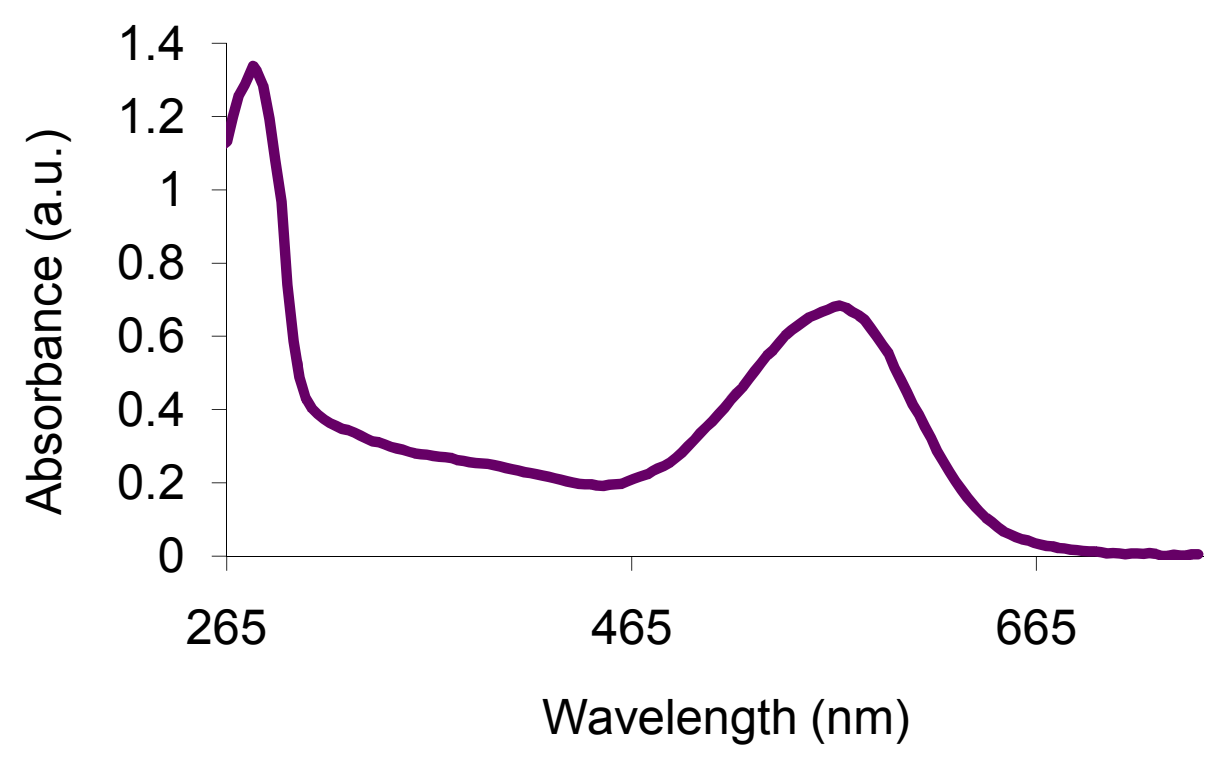

Figure 5. bR absorption spectra.

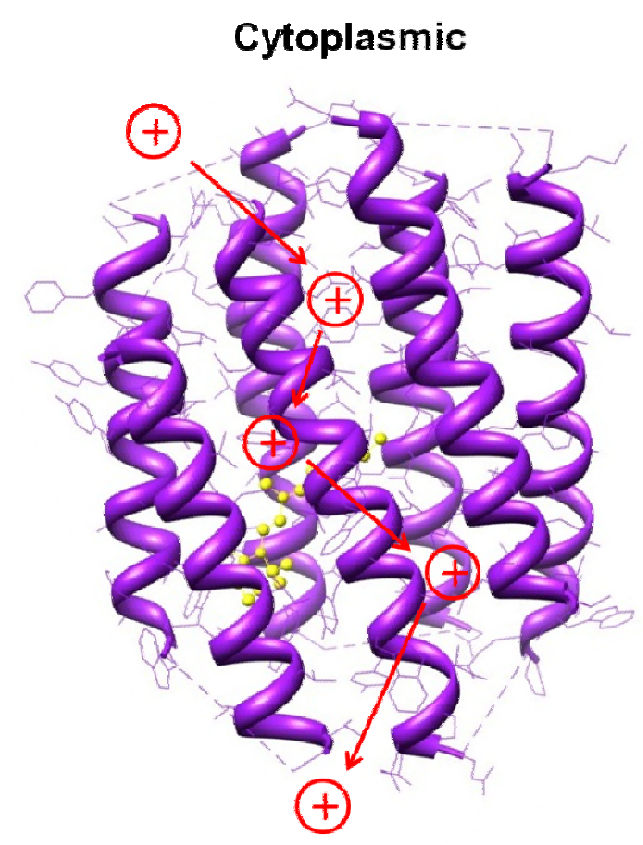

Extracellular

Figure 6. bR proton pumping pathway. 
Upon photon absorption the bR retinal goes through a series of conformational changes to facilitate the proton pumping mechanism [15]. The ion transport within the bR molecule is best described by the Isomerization/Switch/Transport (IST) model [16]. In its ground state, the $\mathrm{bR}_{570}$ retinal is in an all-trans state. The absorption of a photon causes the retinal to isomerize to the 13 -cis form. This photo-induced isomerization is denoted as "I" in the first stage of the bR photocycle displayed in Figure 7 and only takes 500fs. The re-arrangement of the electron structure of the retinal alters the proton affinity of the Schiff base. This ultimately leads to proton release from the Schiff base and subsequent transfer to the Asp- 85 amino acid on the extracellular side in the $\mathrm{L}_{550}-\mathrm{M}_{410}$ intermediate stage. The proton transport is signified by " $\mathrm{T}$ " in Figure 7 and results in release of the original Asp- 85 proton approximately $51 \mu \mathrm{s}$ after photo-illumination. At this point the switch "S" occurs and allows the Schiff base to become accessible by protons on the cytoplasmic side of the membrane. Thus in the $\mathrm{M}_{410}-\mathrm{N}_{560}$ intermediate conversion the Schiff base is reprotonated from the Asp-96 amino acid, which in turn uptakes another proton from the cytoplasm to store for the next photocycle. Following the proton uptake the retinal goes through a thermal isomerization and reverts back to the all-trans state. This switches the Schiff base accessibility back to the extracellular side in the final $\mathrm{O}_{640}$ intermediate and fully returns the bR to its ground state. Although actual proton release can occur as quickly as $51 \mu \mathrm{s}$ after light illumination, the entire photocycle takes approximately $10-15 \mathrm{~ms}$ to complete. Allowing for maximal proton pumping rate on the order of 100 protons/second.

The photocyle of the bR molecule is structurally similar to that present in other rhodopsin ion pump systems. For example the chloride ion pump, halorhodopsin, following a similar photocycle, but its ion affinity is altered due to slight alterations in the amino acid sequence $[17,18]$. In bR, for example, the importance of Asp-96 and Asp-85 in the proton pumping channel was verified by removing these amino acids in a bR mutagen and inhibiting the normal photocycle [19]. The functional similarities of bR to other ion pumps could allow for easy integration of alternate ion pump systems into technology developed for $b R$. 


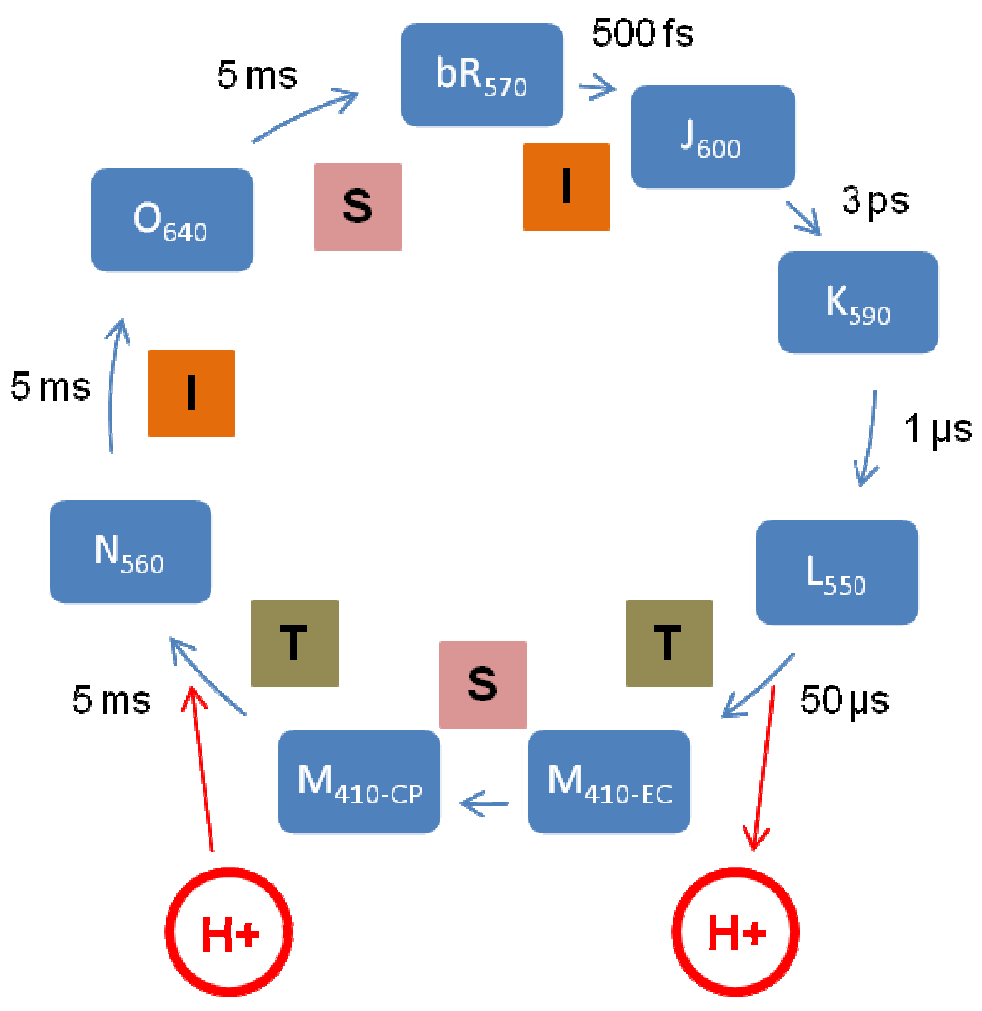

Figure 7. Proton-pumping cycle of $b R$, demonstrating the photocycle intermediates and associated lifetimes.

\subsubsection{Production and Purification of bR}

For engineered applications, $\mathrm{bR}$ is processed into membrane patches, known as purple membrane (PM). Purple membrane is simply a large cell membrane patch, on average $500 \mathrm{~nm}$ in diameter, which is composed of multiple bR molecules and their associated lipids. It is called PM due to its distinct purple color, which is due to the absorption properties of the bR molecules.

The process of preparing and purifying bacteriorhodopsin for engineered applications has been extensively described [20]. In brief, the $H$. halobium cells are grown at $40^{\circ} \mathrm{C}$ in a basal salts medium containing bacteriological peptone. Once grown to a desired density, the cells are collected by centrifugation at 17,700g for 10 minutes. The collected cells 
are then lysed through the addition of deionized water and the released nucleic acids are digested with 1-3Units/mL, final solution volume, DNase I. The larger cell debris is collected through centrifugation at $3,000 \mathrm{~g}$ for 5 minutes. Purple membrane remains in the supernatant and is separated from the red membrane by centrifugation at $105,000 \mathrm{~g}$ for 30 minutes. The purple membrane will be pelleted in this centrifugation step, which is repeated until the supernatant is clear. For additional purification, passing the purple membrane over a linear sucrose gradient is also recommended.

\subsubsection{Applying bR to Engineered Applications}

Purple membrane has many unique properties that make it a viable engineering material. Purple membrane is composed of bR and its associated lipids in a two-dimensional crystal, which provide its high degree of chemical stability and resistance to thermal degradation. Specifically, PM has been shown to maintain functionality at temperatures up to $80^{\circ} \mathrm{C}$ in water and $140^{\circ} \mathrm{C}$ dried [21]. In the dried state, as well as the wet state, PM retains its light absorption properties and photochemical activity for years.

The potential applications for PM in engineered devices are extensive, including information storage, information processing, charge transport membranes, and photoelectric systems [21]. For many of the applications, such as photoelectric systems, it is necessary to orient the PM during deposition. This is necessary to facilitate a unidirectional transport of protons within the layered system, producing a larger electrical signal. Techniques such as Langmuir-Blodgett (LB), immunochemical binding, and electrophoretic sedimentation (EPS) may generate a high degree of PM orientation upon deposition, although quantitative studies are lacking.

The Langmuir-Blodgett and immunochemical binding techniques allow the creation of PM monolayers with a high degree of orientation. Currently, however, the most common technique for PM orientation is electrophoretic sedimentation. Electrophoretic sedimentation creates a thicker PM layer relative to both LB and immunochemical 
techniques, often resulting in thousands of layers of PM. The process of orienting PM through electrophoretic sedimentation has been extensively analyzed to maximize the bR electrical output for the final engineered system.

Electrophoretic sedimentation is a common technique used to orient biological and organic macromolecules based on their charge. As shown in Figure 8, the extracellular side of the bR molecule has a strong negative charge at neutral $\mathrm{pH}$, while the cytoplasmic side is relatively positive.

Cytoplasmic -Positive Dipole-

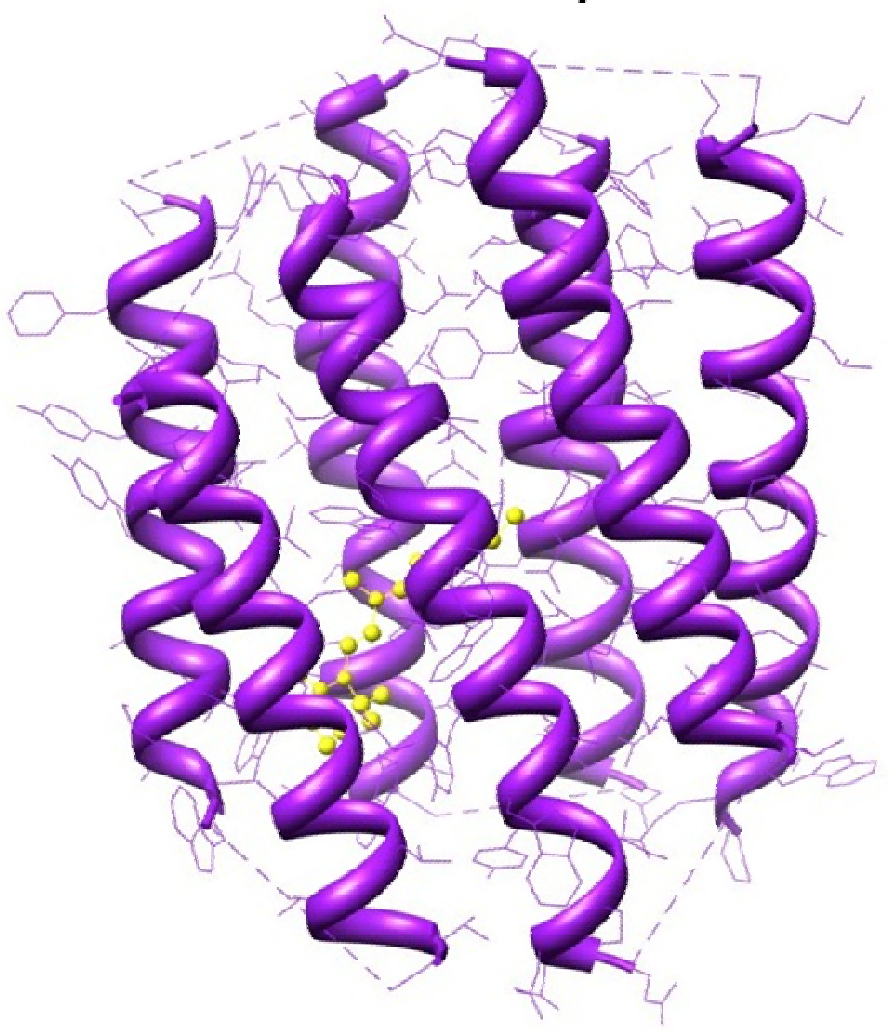

Extracellular -Negative Dipole-

Figure 8. Charge of bR molecule at neutral pH.

13 
For EPS of PM, a suspension of PM is placed between two electrically conductive plates and a voltage gradient is applied across the two plates. With a PM suspension near neutral $\mathrm{pH}$, the extracellular side will preferentially orient and attach to the plate serving as the anode. Typically an indium tin oxide [22] electrode serves as the anode, which allows light to reach the $b R$ molecules in the final system.

Widely varying PM concentrations have been used for EPS, ranging from $4 \mathrm{mg} / \mathrm{mL}$ [23] to $15 \mathrm{mg} / \mathrm{mL}$ [24]. Separation distances between the conductive plates of $0.5 \mathrm{~mm}-2 \mathrm{~mm}$ [25] have been shown to be effective. It is common practice to apply a voltage gradient between $20-40 \mathrm{~V} / \mathrm{cm}$ of separation for 60 seconds to produce sufficient PM orientation [25], but deposition times between 10 seconds [26] and 5 minutes [24] in that voltage range have also been used to produce functional PM films. A study optimizing the output current of PM films versus deposition voltage found that an applied voltage of $3.9 \mathrm{~V}$ for 60 seconds maximized PM functionality [27]. The electrode separation distance in this study was only $0.089 \mathrm{~mm}$, implying an optimal voltage gradient of $438 \mathrm{~V} / \mathrm{cm}$. Other research groups have applied voltages of $3.9 \mathrm{~V}$ with a $1 \mathrm{~mm}$ separation distance ( $\sim 39$ $\mathrm{V} / \mathrm{cm}$ ) to achieve PM deposition and orientation. Although no specific relationship between spacer distance and PM electrical output has been reported, these articles suggest that spacer distance is not critical and that an applied gradient of $\sim 4 \mathrm{~V} / \mathrm{mm}$ is sufficient.

After the PM has been deposited onto the anode, it is gently rinsed in deionized/distilled water for 15 seconds to remove any loosely bound PM or contaminants. The PM film and electrode are then dried for $\sim 24$ hours prior to application. The drying process is important since the water content of the PM film affects the photocycle of the $b R$ molecules [28]. Dehydration of the PM film has been shown to slow the photocycle along with decreasing the peak output voltage [23]. Therefore, the PM films are typically dried in a humidity chamber set to a relative humidity of $\sim 51 \%$ to prevent extensive dehydration and cracking of the sample [29]. After drying, a conductive plate is attached to the top of the PM film, often using a spacer to prevent any contact between the top and bottom plates. The use of an electrolyte gel, such as $100 \mathrm{mM} \mathrm{KCl}$ gel, between the top 
electrode and the PM film has been shown to ensure a good connection [30, 31]. The final electrode is then connected to an amplification circuit for signal measurement. An example photovoltage output is shown in Figure 9.

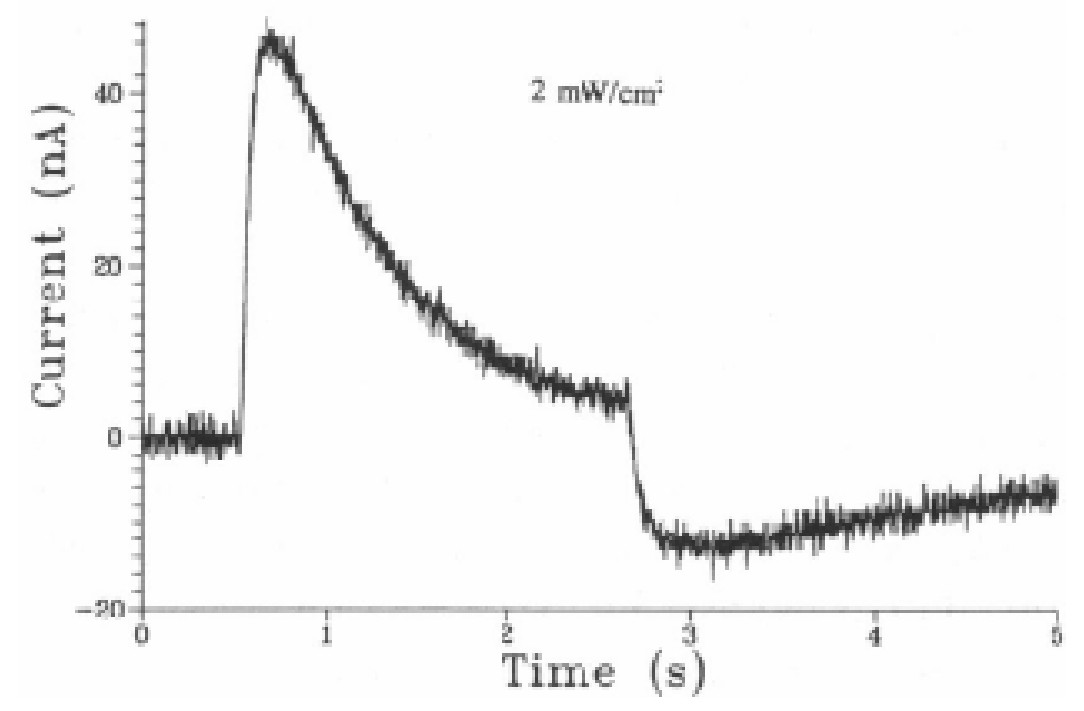

Figure 9. Sample bR film electrical output upon light illumination [30].

As shown in Figure 9, when the light is turned on there is a sharp spike in the photocurrent output of an EPS oriented bR-based electrode. The measured current is caused by the movement of protons in one-direction, towards the extracellular side of the protein. The protons aren't pumped out of the extracellular side of the protein during $b R$ excitation in dried samples, but shifted to that side [24]. The protons are held in that shifted position for the duration of the illumination. When the illumination is switched off, the protons shift back towards the cytoplasmic side of the protein, resulting in the negative photocurrent spike. The photoelectric activity of oriented PM films has been studied for a number of applications, including incorporation into solar cells [32] and imaging devices $[27,33,34]$.

Current bR-based optical sensors typically sandwich the PM film between two solid conductive plates. However, PM has been utilized for integrated circuit applications. Specifically, research has shown that the photoelectric activity of PM films can be 
captured with GaAs field effect transistors (FET) [35]. The PM film was deposited and dried, non-oriented, on the Au gate of the GaAs FET and covered with an ITO film. A schematic of the entire bR-FET setup is shown in Figure 10. The resulting PM films were approximately $1 \mathrm{~mm}$ in diameter, $100 \mu \mathrm{m}$ thick, and had a resistivity of $\sim 10^{11} \Omega$-cm. The resulting electrical output of the amplifier system under $\mathrm{He}-\mathrm{Ne}$ laser light illumination is shown in Figure 11.

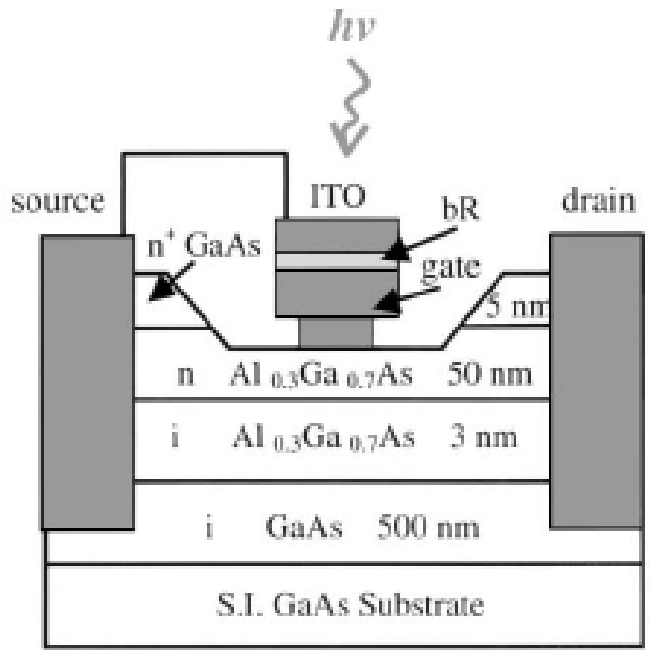

Figure 10. Schematic of FET with integrated PM film [35]. Permission received from the Optical Society of America and can be found in Appendix A.3.

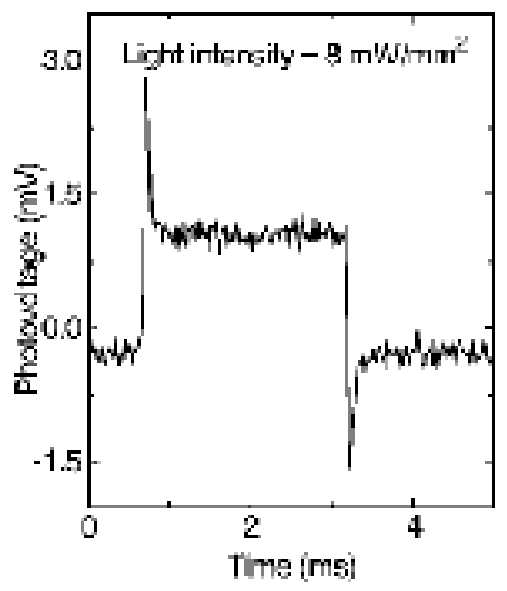

Figure 11. Photovoltage output of unoriented PM film integrated with GaAs FET [35]. Permission received from the Optical Society of America and can be found in Appendix A.3. 
As with the aforementioned integration of PM with an FET, current research with bR electrodes typically utilized PM films with diameters larger than $1 \mathrm{~mm}$. The ability to apply PM films on the micron-scale will be necessary for certain advanced sensing applications. The ability to pattern PM films on the micron scale has been shown by combining soft-lithography techniques and EPS [36]. The basic process for micron-scale patterning of PM is shown in Figure 12.

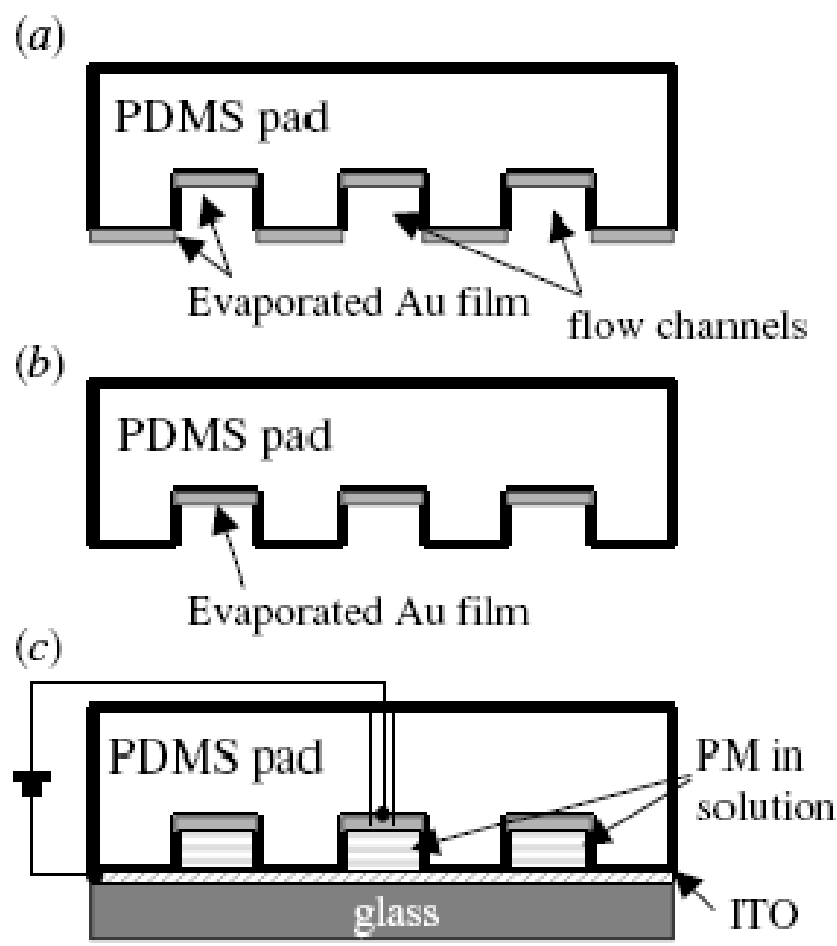

Figure 12. Method to pattern PM on the micron-scale [36]. Permission received from IOP publishing and can be found in Appendix A.4.

Soft-lithography is used to produce a polydimethylsiloxane (PDMS) pad with micro-scale channels. A 70nm gold layer is thermally evaporated onto the surface of the pad to serve as the cathode during EPS. The pad is placed onto the ITO surface and small channels allow $5 \mathrm{mg} / \mathrm{mL}$ PM solution to fill the micron-scale cavities of the PDMS pad. A 3-5V DC potential is applied between the ITO and gold surfaces for 5-30 seconds to orient and deposit the PM. Following deposition, the PDMS is removed and the ITO electrode with 
patterned PM is rinsed with Millipore ${ }^{\mathrm{TM}}$ water. The final patterned PM is $10 \mu \mathrm{m}$ squares that are between 4-9 monolayers thick depending upon the initial PM solution concentration and voltage application time. A scanning force microscopy (SFM) image showing the final PM patterning is shown in Figure 13.

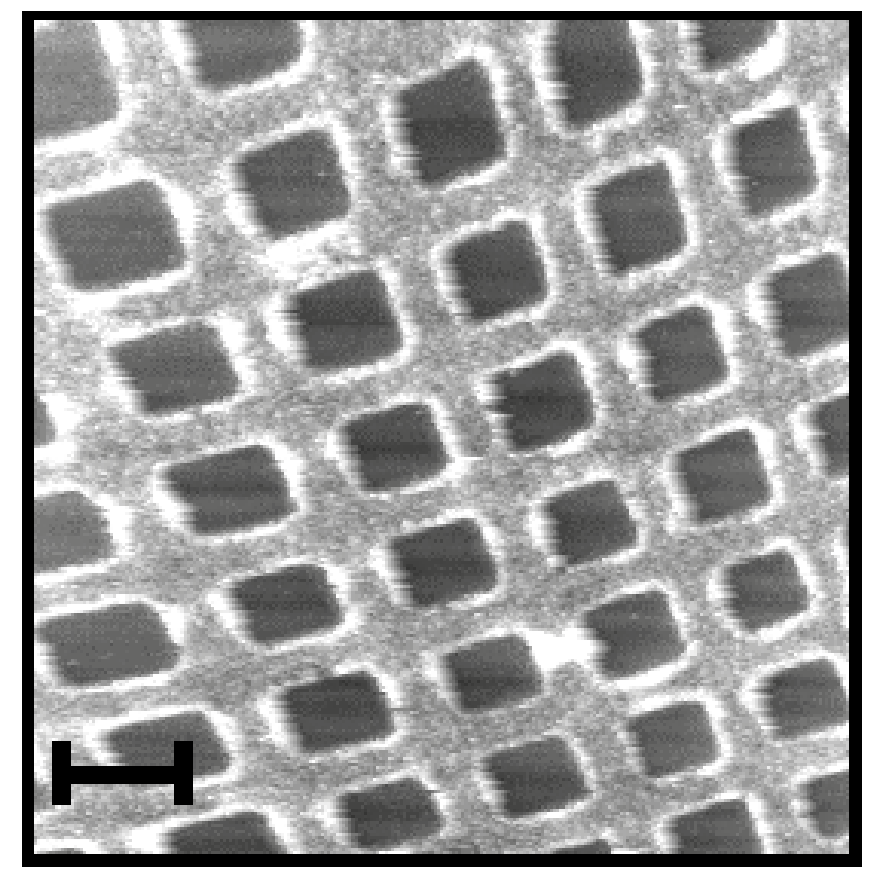

Figure 13. SFM image of micro-scale patterned PM (scale bar $10 \mu \mathrm{m}$ ) [36]. Permission received from IOP publishing and can be found in Appendix A.4.

To date, there is no published literature pertaining to the construction of bR-based electrodes at the sub micron-scale. One obstacle to producing nano-scale bR-based electrodes is the size of the PM fragments, which average $\sim 500 \mathrm{~nm}$ in diameter. Individual bR molecules have dimensions on the order of $6.3 \mathrm{~nm} \times 6.3 \mathrm{~nm} \times 4.9 \mathrm{~nm}$ thick; therefore the ability to break-down PM fragments and apply individual bR molecules or smaller groups of $\mathrm{bR}$ molecules will be important for nanoscale applications. Using detergents, such as Triton X-100, or octyl-glucoside, the production of bR monomers from large PM fragments has been achieved while retaining bR physical properties [37, 38]. 


\subsection{QUANTUM DOTS}

Quantum dots (QD) are semiconductor particles that have dimensions on the order of nanometers, typically $2 \mathrm{~nm}-10 \mathrm{~nm}$. These QDs can be composed of a wide-array of IIVI, III-V, and IV-VI materials depending on the application; including CdSe, CdS, CdTe, InAs, and PbS [39, 40]. Due to their small dimensions, well below the exciton Bohr radius, QDs have discrete energy levels and their bandgap can be fine tuned based on the size of the dot. Since the size of the bandgap determines the energy of the electromagnetic radiation given off by the electron when it falls back to the valence band, the wavelength emitted by the QD can be directly controlled by its size [41]. Along with providing narrow emission spectra, QDs have a broad absorption range that peaks in the ultra-violet (UV) region. Therefore a multitude of QDs with different emission wavelengths can be excited simultaneously from a single excitation source. The quantum dots referred to in this paper are colloidal semiconductor QDs, which are composed of a $\mathrm{CdSe}$ core with a $\mathrm{ZnS}$ protective shell. The smaller the size of the QD, the more blueshifted the absorption and emission peaks are.

When compared to other fluorescing materials, such as fluorescent dyes, QDs have vastly improved characteristics such as quantum yield, chemical stability, lifetime, ease of emission tunability, broad absorption range, and a greater resistance to photo-bleaching [42]. Apart from their intrinsic properties, the surface chemistry of QDs has also been shown to be amenable to the addition of functionalized binding materials, thus allowing the use of QDs in a wide array of applications. With their biocompatible coatings and narrow emission spectra, QDs primary application to date has been in vivo biological targeting for imaging and medical diagnostics [43, 44]. Additionally since QD emission can be easily tuned based on size, multiplexed detection has been shown feasible by coordinating specific target recognition for a multitude of different sized QDs [45]. Aside from their applications in biological systems, several technological developments have been made utilizing the quantum mechanical properties of QDs. Briefly, these 
include solar cells [46-48], quantum computing [49], single electron transistors [50], and diode lasers [51, 52].

\subsubsection{Quantum Dot Synthesis}

The synthesis of semiconductor colloidal quantum dots (QDs) has been well documented [40, 53-56]. Depending on the application, QDs can be created that span the near ultraviolet, visible, and near infra-red photonic wavelength regions. Photonic emission in these spectrums can be achieved through $\mathrm{CdS}, \mathrm{CdSe}$, and CdTe core QDs, respectively [40]. The QD emission can be fine-tuned to the desired wavelength based on the diameter of the QD nanocrystal, with smaller diameter implying blue-shifted wavelengths and larger diameters creating red-shifted wavelengths in their respective spectral regions. The typical spectral ranges covered by each QD core type are displayed in Figure 14.

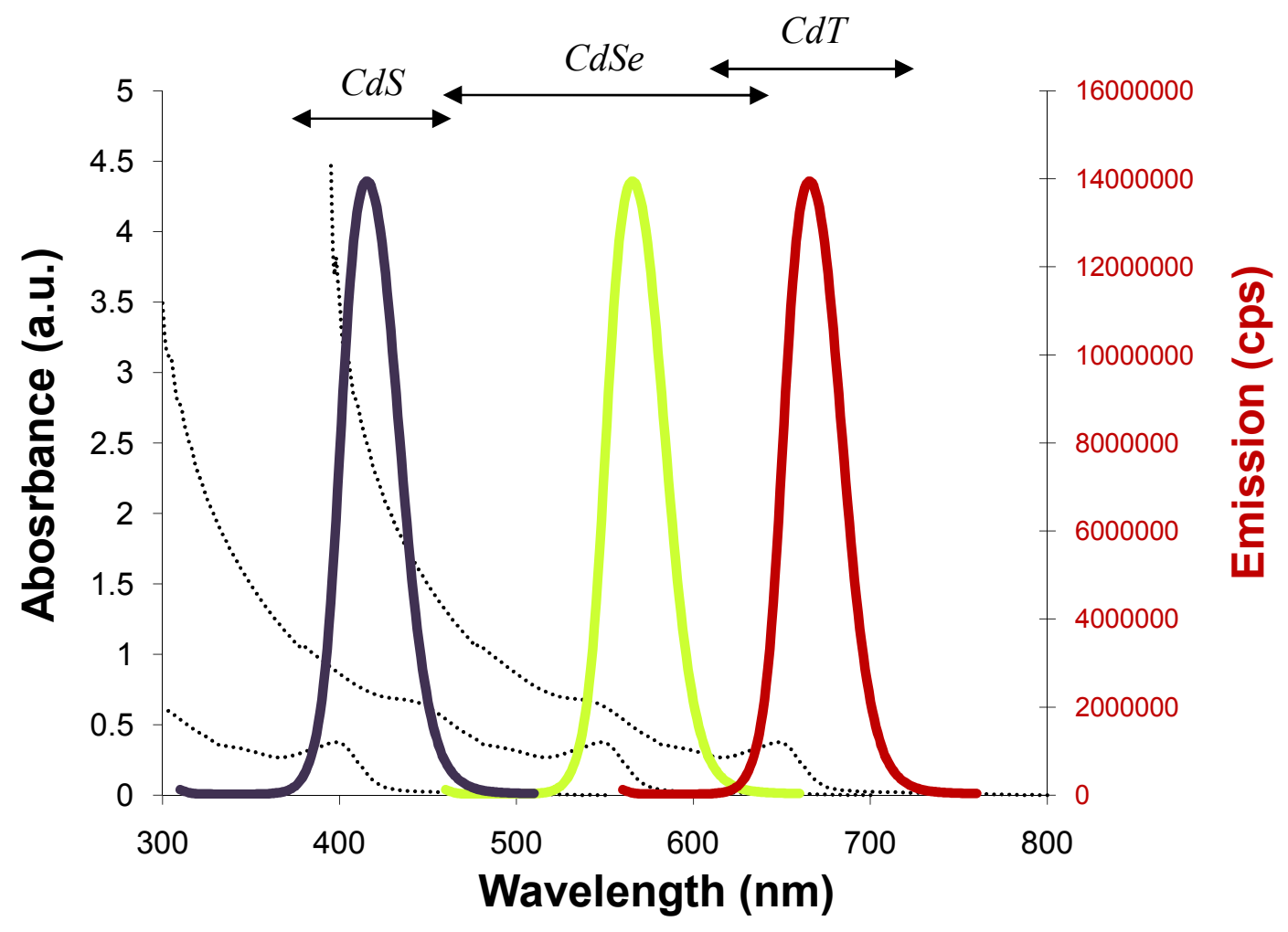

Figure 14. Typical spectral emission ranges for synthesized CdS, CdSe, and CdTe core QDs. 
An example of CdSe QD synthesis consists of 0.0514g Cadmium Oxide (CdO) and $3.7768 \mathrm{~g}$ of trioctylphosphine oxide (TOPO) are loaded into a $25 \mathrm{~mL}$ flask. Under Argon flow, the mixture is heated to $300-320^{\circ} \mathrm{C}$. This allows for the $\mathrm{CdO}$ to fully dissolve into the TOPO and results in a colorless solution. Once dissolved, the solution is cooled to $270^{\circ} \mathrm{C}$ and the desired non-metal (Sulfur (S), Selenium (Se), or Tellurium (Te)) is injected into the flask. Prior to injection the non-metal is prepared by dissolving 0.00052 moles $(0.0167 \mathrm{~g} \mathrm{~S}, 0.0411 \mathrm{~g} \mathrm{Se}$, or $0.0664 \mathrm{~g} \mathrm{Te})$ into $2 \mathrm{~g}$ trioctylphosphine (TOP). Following injection the solution is cooled to $250^{\circ} \mathrm{C}$, at which point the QDs begin to form. The QD diameter is determined by the length of time the solution is kept at $250^{\circ} \mathrm{C}$, with longer time periods resulting in larger diameters. After the desired synthesis time the solution is cooled to room temperature to halt nanocrystal growth. Typical growth times will range from 10 seconds to 2 hours. Once cooled the QDs are precipitated out of the solution by the addition of anhydrous methanol followed by centrifugation. The QDs are redispersed in anhydrous methanol and centrifuged three times. The QDs are finally dissolved in chloroform or toluene for storage.

In many cases it is desirable to 'passivate' the QDs by attaching a polymeric shell on top of the QD core. This shell has demonstrated the ability to increase the QDs shelf-life, quantum yield, and resistance to oxidation $[57,58]$. The normal core synthesis procedure is followed up to the point at which the nanocrystals have grown to their desired diameter. At this point $2.5 \mathrm{~mL}$ of $\mathrm{Zn} / \mathrm{S} /$ trioctylphosphine [59] solution (composed of $0.0025 \mathrm{~mol}$ Bis(trimethylsilyl) sulfide, $0.0035 \mathrm{~mol}$ Diethylzinc, and $20 \mathrm{~mL}$ TOP) is added to the nanocrystal solution. Once injected the entire mixture is cooled to $100^{\circ} \mathrm{C}$ and stirred for 1 hour. Following growth the core/shell QDs are purified by the aforementioned method. 


\subsubsection{Modifying the Quantum Dot Surface Chemistry}

The primary use of QDs to date is to tag biomolecules for imaging [60, 61]. To use QDs in a biological environment, the surface must first be coated to make it water soluble and for protection against oxidative effects. Plain CdSe/ZnS QDs are suspended in non-polar hydrophobic solvents such as chloroform or toluene to prevent oxidation. When exposed to ambient air or water, the outer CdSe layer oxidizes to produce $\mathrm{SeO}_{2}$, which is nonemissive [62]. Initially, the oxidation of the outer CdSe layer results in a blue-shift of the QD emission, which is due to the 'shrinking' of the functional QD diameter. The blueshift of the emission continues, as shown in Figure 15, until the QD luminescence disappears completely.

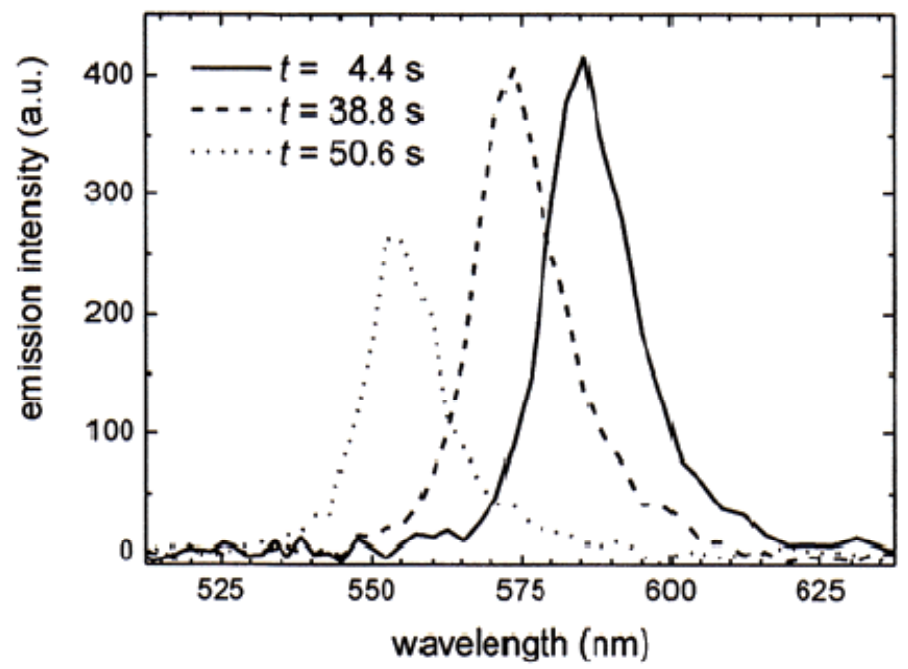

Figure 15. Blue-shift of 585nm emission CdSe/ZnS QD upon oxidation [58].

In single QDs, the loss of functionality can occur in a matter of minutes depending on the thickness of the $\mathrm{ZnS}$ layers. It has been shown that increasing the $\mathrm{ZnS}$ shell thickness from 4 to 7 monolayers increases the QD lifetime from 2.5 to 3.5 minutes, respectively [58]. The effective diameter of $585 \mathrm{~nm}$ emission QDs shrinks from $5 \mathrm{~nm}$ to $4 \mathrm{~nm}$, a loss of the outer 2 CdSe layers, during this time [63]. To reduce the effects of oxidation, the use of a protective lipid or polymeric shell over the $\mathrm{ZnS}$ layer has been employed. One 
common coating used is polyethylene glycol (PEG), which slows oxidative effects, makes the QDs water soluble, and helps prevent unwanted binding of foreign molecules to the QD in bio-applications [44].

The main advantages of using QDs, rather than traditional fluorophores in bio-tagging applications, is not just their resistance to photobleaching and ease of tunability, but the ability to add molecules, such as ligands, to the QD surface without affecting QD functionality; which isn't always the case for fluorophores. The surfaces of CdSe core/ZnS shell QDs have the ability to be functionalized with a wide array of molecules. Evident Technologies, for example, provides QDs with surface coatings of amine or carboxyl groups [64]. This allows for the conjugation of virtually any biomolecule to the QD surface. Evident also provides QDs already conjugated to select antibodies, biotin, or streptavidin. All of Evident's QDs which are engineered for bio-applications have a PEG lipid layer on top of the $\mathrm{ZnS}$ shell for increased biocompatibility.

Using surface-functionalized QDs, researchers have been able to selectively target molecules for QD attachment. An example of such targeting has been shown by the attachment of maltose binding protein (MPB) to the QD surface. Through thiol coordination, a dihydrolipoic acid (DPHA) monolayer can be attached to the outer $\mathrm{ZnS}$ layer of the QD. The addition of MBP with an expressed pentahistidine segment at its Cterminus (MPB-5HIS) has not only been shown to electrostatically bind to the DPHAcapped QD, but also increases the QD photoluminescence intensity [41]. Techniques to use this bioconjugated QD for the detection of maltose primarily entail the use of FRET.

\subsection{FLUORESCENCE RESONANCE ENERGY TRANSFER}

A detection technique known as fluorescence resonance energy transfer (FRET), also referred to as Forster resonance energy transfer, has been characterized and optimized through biological sciences research over the past several decades. Primarily used as a 
'molecular ruler,' the measurement of FRET between two molecules has proven to be a very sensitive technique for measuring Angstrom-level separation distances [65], molecular imaging [66], and detecting the presence of a target molecules [67].

The basic fundamentals of FRET are that when the emission spectra of a fluorescing molecule, termed the donor, overlaps to some degree with the absorption spectra of an acceptor molecule, the energy from the excited-state donor molecule can be transferred non-radiatively to the acceptor molecule. Therefore some of the energy that would have been given off by the donor molecule through electromagnetic radiation is now transferred to the acceptor molecule by resonance through the dipole moments of the two molecules. The degree of FRET seen in a specific donor/acceptor pair is directly related to their separation distance, donor emission and acceptor absorbance spectral overlap, and orientation of the donor/acceptor dipole moments.

In order to calculate the FRET efficiency between a donor and acceptor, the Forster radius must first be determined. The Forster radius is defined as the distance between the donor and acceptor where $50 \%$ of the donor's energy is transferred via FRET. The Forster distance $\left(\mathrm{R}_{\mathrm{o}}\right)$ is defined as:

$$
\mathrm{Ro}^{6}=\left(8.8 \times 10^{23}\right)\left(\kappa^{2}\right)\left(\eta_{\mathrm{D}}^{4}\right)\left(\Phi_{\mathrm{D}}\right) \mathrm{J}(\lambda)
$$

Where $\kappa$ is the dipole orientation factor, $\Phi_{\mathrm{D}}$ is the quantum yield of the donor, $J$ is the normalized overlap integral between the donor and acceptor, and $\eta$ is the refractive index of the medium. Most of these variables have measurable values, but the $J$-integral must be calculated using the equation:

$$
J=\int \mathrm{f}_{\mathrm{D}}(\lambda) \varepsilon_{\mathrm{A}}(\lambda) \lambda^{4} d \lambda
$$

Where $f_{D}$ is the peak normalized fluorescence spectrum for the donor, $\varepsilon_{A}$ is the molar absorption coefficient of the acceptor, and $\lambda$ is the wavelength. 
After the Forster radius is determined, the FRET efficiency of the donor/acceptor pair at various separation distances can be determined. The FRET efficiency indicates the percentage of non-photonic emission from the donor, which is also the percentage of energy transferred to the acceptor through electron resonance. FRET efficiency (E) can be calculated using the equation:

$$
\mathrm{E}=\left(\mathrm{R}_{\mathrm{o}}{ }^{6}\right) /\left(\mathrm{R}_{\mathrm{o}}{ }^{6}+\mathrm{R}^{6}\right)
$$

Where $\mathrm{R}_{\mathrm{o}}$ is the Forster radius for $50 \%$ resonance transfer and $\mathrm{R}$ is the actual separation distance between the donor and acceptor molecules being studied.

\subsection{1. bR as a FRET Acceptor}

Research has shown that bR molecules have the ability to participate in FRET coupling. The retinal of the rhodopsin protein, including bR, has a strong absorption band which makes it a viable FRET acceptor [68-70]. To date, FRET techniques with bR have primarily been used as a 'molecular ruler,' measuring the affinity that $b R$ molecules have for various lipid groups.

In a study analyzing the ability of bR to sort lipids based on their degree of hydrophobicity, a 7-nitrobenz-2-oxa-1,3-diazol-4-yl (NBD) fluorophore served as the FRET donor [70]. The overlap of the NBD fluorophore emission and bR retinal absorption is shown in Figure 16. 


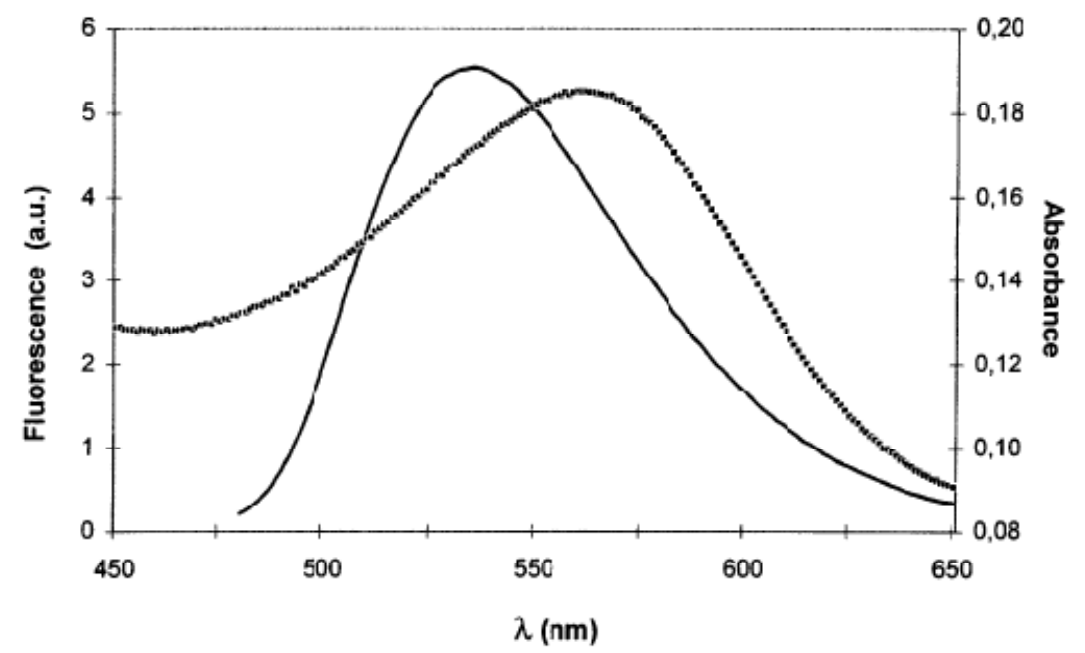

Figure 16. Overlap of NBD emission (solid line) and bR Retinal Absorption (dotted line) [70].

Although the emission and absorption peaks differ by approximately $35 \mathrm{~nm}$, a strong overlap still exists, with the J-integral calculated to be $3.58 \times 10^{-13} \mathrm{M}^{-1} \mathrm{~cm}^{3}$. At room temperature, the $\mathrm{R}_{\mathrm{o}}$ (Forster Radius) equation variables were set to,

- $\kappa^{2}=2 / 3 \quad$ (orientation factor)

- $\Phi_{\mathrm{D}}=0.254 \quad$ (quantum yield of the donor)

- $\eta=1.33 \quad$ (refractive index of water)

leading to a calculated $R_{o}$ value of $5.04 \mathrm{~nm}$, implying that at a separation distance of $5.04 \mathrm{~nm}$ between the NBD fluorophore and the bR retinal, the NBD fluorophore photonic emission would be quenched by $50 \%$. The experimental values determined through this study approximated their theoretical values calculated using these FRET parameters. The results of this study also show that the range of energy transfer can extend far into the lipid phase and couple with the retinal attached at the center of the bR molecule.

Further, the bR retinal can FRET couple with fluorophores that provide less emission/absorption spectra overlap [69]. To study energy transfer theories in twodimensional lipid systems, the bR retinal was FRET coupled with a donor 1,6-diphenyl1,3,5-hexatriene (DPH) fluorophore. The DPH fluorescence emission peaks around 
$425 \mathrm{~nm}$, far short of the $570 \mathrm{~nm}$ absorption maxima of the bR retinal, but the broad emission manages to provide an overlap integral of $\mathrm{J}=7.3 \times 10^{-14} \mathrm{M}^{-1} \mathrm{~cm}^{3}$. The $R_{0}$ (Forster Radius) equation variables were set to,

- $\kappa^{2}=0.44 \quad$ (orientation factor)

- $\Phi_{\mathrm{D}}=1 \quad$ (quantum yield of the donor)

- $\eta=1.44 \quad$ (refractive index of medium)

leading to a calculated $R_{o}$ value of $4.3 \mathrm{~nm}$. It was determined through this study that the fluorescence of the donor is progressively quenched with increasing amounts of bR in the membrane, thus increasing the acceptor: donor ratio increased the FRET efficiency.

In a lipid study using bovine rhodopsin as the FRET acceptor and pyrene (PY) labeled lipids as the donor, the impact of a photobleached rhodopsin retinal on the FRET coupling is made apparent [68]. As displayed in Figure 17, this study utilized the blue shifted absorption spectra of the photo-bleached rhodopsin to achieve FRET coupling.

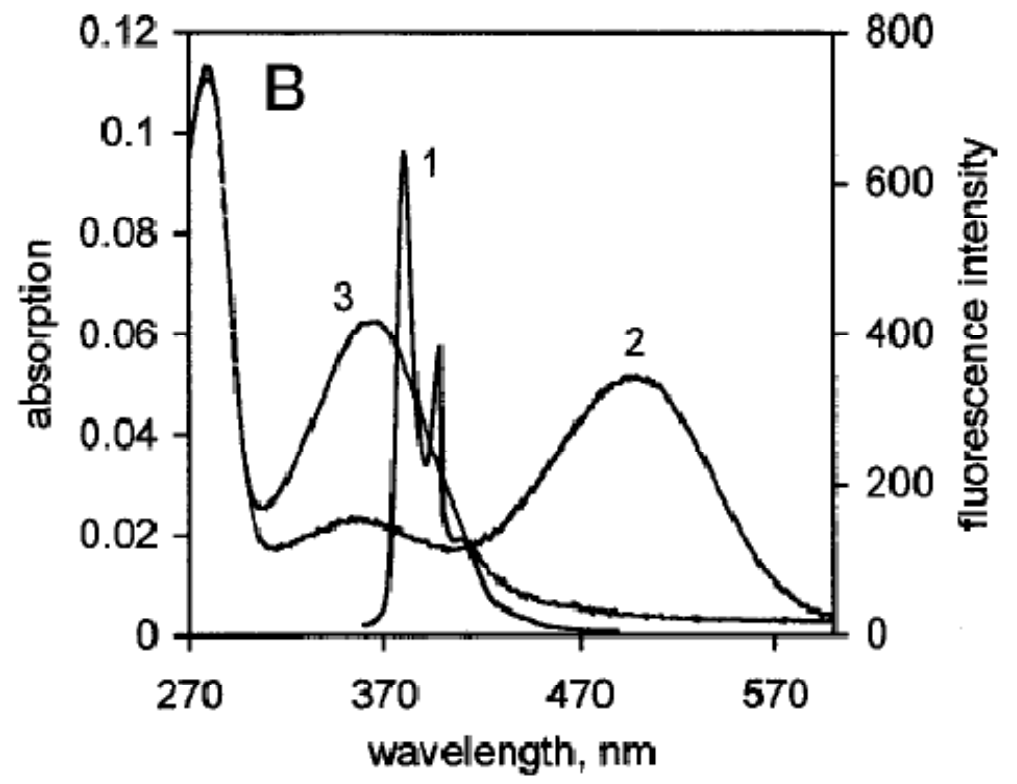

Figure 17. PY emission (curve 1), dark-adapted bovine rhodopin absorption (curve 2) and photobleached bovine rhodopsin absorption (curve 3) [68]. 
Therefore when conducting FRET experiments with the bR retinal serving as the acceptor, care must be made to control and maintain the $\mathrm{bR}$ absorption peak. An extensive study has been published analyzing the effects of constant illumination with the variables of solution $\mathrm{pH}$, illumination light intensity, illumination time, and temperature on the photo-bleaching of bR [71]. Graphs displaying the effects of each of these variables on the absorption spectra of $b R$ are shown in Figure 18.
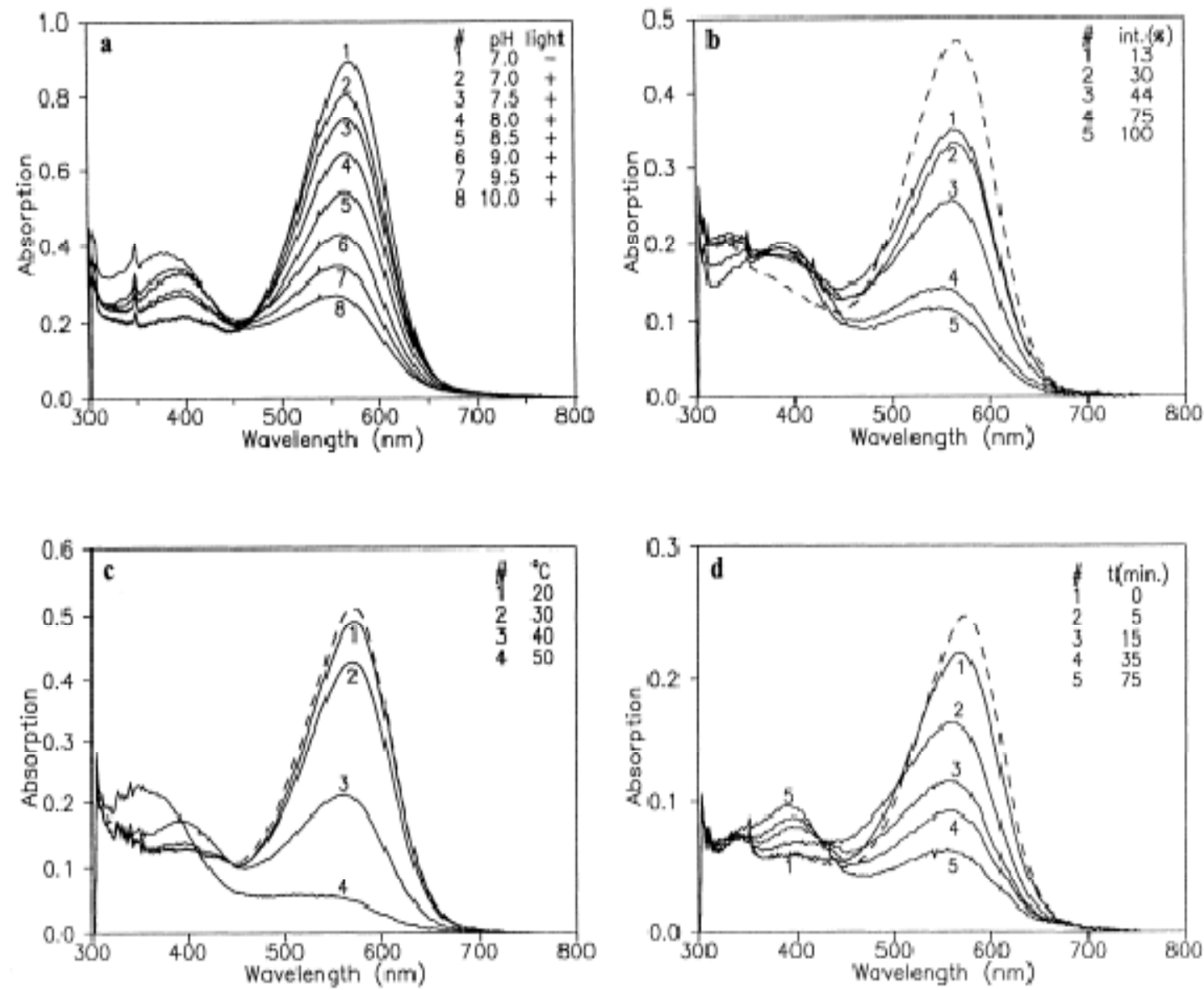

Figure 18. Absorption spectra of bR before and after photo-bleaching by continuous light (a) at different $\mathrm{pH}$ values $\left(50^{\circ} \mathrm{C}, 30 \mathrm{~min}\right.$ illumination at $\left.400 \mathrm{~mW} / \mathrm{cm}^{2}\right)$, (b) at different percentages of maximum illumination power of $400 \mathrm{~mW} / \mathrm{cm}^{2}\left(50^{\circ} \mathrm{C}, \mathrm{pH} 9.5,30 \mathrm{~min}\right.$ illumination), (c) at different temperatures (pH 9.5, 60min illumination at $\left.400 \mathrm{~mW} / \mathrm{cm}^{2}\right)$, and (d) at different bleaching times $\left(50^{\circ} \mathrm{C}\right.$, pH 9.5, 400mW/cm² illumination power) [71]. Reprinted from FEBS Letters, Vol. 450, Dancshazy et al., Bleaching of Bacteriorhodopsin by Continuous Light, p. 154-157, 1999, with permission from Elsevier [OR APPLICABLE SOCIETY COPYRIGHT OWNER] and can be found in Appendix A.5. 
For this study a high pressure mercury lamp with optical and heat filters was used as the illumination source. From Figure 18 several interesting conclusions can be made. First, is that increasing the solution $\mathrm{pH}$, light intensity, temperature, and illumination time will increase the degree of photobleaching of the bR retinal under constant illumination. It can also be noted that the reduction in retinal absorbance at $570 \mathrm{~nm}$ corresponds to an increase in absorbance around 400nm upon photobleaching, without any intermediate absorption peaks. Therefore to maximize the absorption spectra of the bR retinal at 570nm for FRET coupling, care must be taken to keep the solution around $\mathrm{pH} 7$, close to room temperature, and to limit the time and intensity of light illumination on the sample.

Although the electrical output of a bR-based electrode is directly related to the degree of light activation, there are no known studies to date evaluating the relationship between PM absorbance and electrical activity.

\subsubsection{Quantum Dots as a FRET Donor}

Quantum dots have the ability to serve as a FRET donor with a wide array of acceptor particles. Primarily, researchers have used fluorophores to serve as the FRET acceptor for the QD. In the case of the aforementioned MBP functionalized QD, the detection of maltose using FRET has been achieved in several different ways. One method is to initially fill the disaccharide binding sites of the MBP with $\beta$-cyclodextrin-acceptor dyes $(\beta-C D)$ bound to a suitable fluorophore to serve as a FRET acceptor to the QD, such as the QSY9 fluorophore with 560nm emission QDs [41]. The QSY9 fluorophores quench the 560nm QD, with the degree of quenching related to the number of QSY9-labeld MBPs per QD. As schematically represented in Figure 19, the presence of maltose will displace the QSY9, since maltose has a stronger binding affinity of $3.50 \mu \mathrm{M}$ compared to $1.50 \mu \mathrm{M}$ for $\beta$-CD. The displacement of the quenching QSY9 molecules will increase the photonic emission of the QD. 


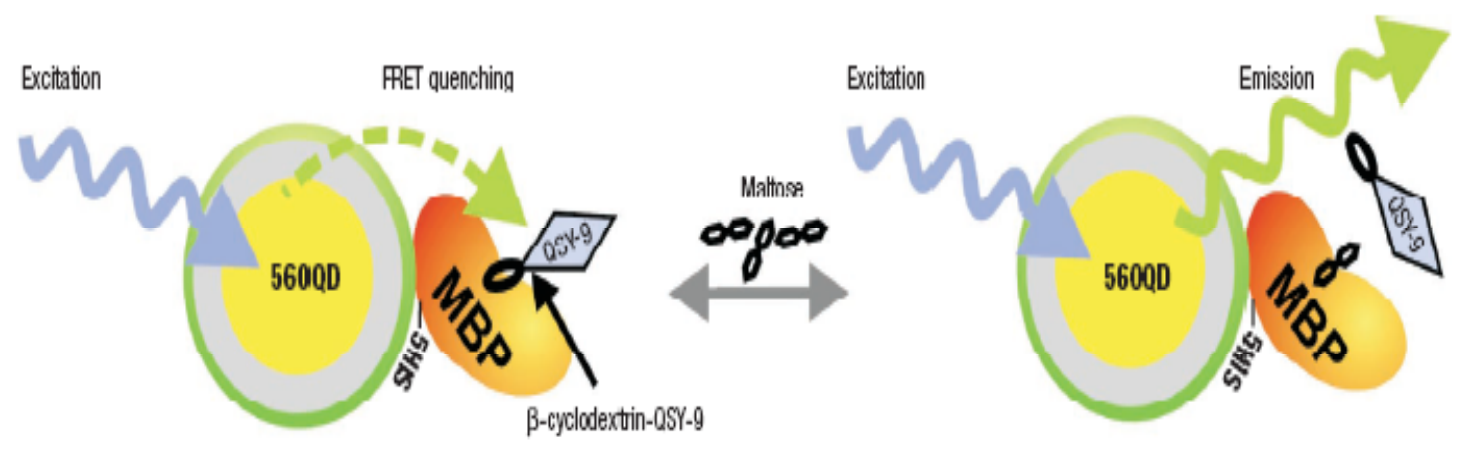

Figure 19. Schematic of maltose displacement of $\beta$-CD-QSY9 in a MBP-5HIS/QD bioconjugate [41]. Reprinted by permission from Macmillan Publishers Ltd: Nature Materials, Medintz, I.L., et al., Self-assembled nanoscale biosensors based on quantum dot FRET donors, 2: p. 630-638, copyright (2003) and permissions can be found in Appendix A.6.

Maltose may also be directly labeled with an acceptable FRET acceptor, thus upon binding of the maltose to the MPB the QD emission will be quenched [72]. Materials other than organic dyes and fluorophores are also able to FRET couple with QDs. Certain metallic surfaces, when in close proximity to CdSe QD's, affect the QD emission. Specifically, it was found that when in contact with smooth gold surfaces, the QD emission was quenched, but while in contact with a rough gold surface the QD emission was increased 5-fold [73] due to surface enhanced exciton fluorescence. The rough gold surface used in this study had been prepared on silicon wafers, with titanium (20nm) and gold (120nm) deposited by thermal evaporation. Peaks and valleys were created on the surface through electrochemical etching, which produced an average roughness of $30 \mathrm{~nm}$.

Quenching of QD emission, as with other organic dyes, is due to non-radiative energy transfer from the QD to the gold. The dipole interactions between the QD and a rough gold surface is proposed to increase the lifetime of the QD fluorescence, thus the ionization mechanism that usually results in the blinking behavior of a QD now provides measurable electromagnetic radiation [73]. The degree of dipole interaction appears to determine whether gold has a quenching or amplifying effect on the QD emission. 
Research groups have exploited the interactions between gold and QDs for biosensing applications. FRET coupling occurs when a gold nanoparticle comes in close contact with a 540nm emission QD. By attaching complimentary DNA strands to the gold nanoparticle and QD, the two particles can be brought into close contact by hybridization of the DNA strands [74]. The QDs were functionalized with carboxylic acid and chemically treated to facilitate a linkage to the 5'-end of a DNA sequence. Mixing the Au-DNA ( $\mathrm{Au}$ attached to 3'-end) with the QD-DNA (QD attached to 5'-end) at a 1:1 ratio in an aqueous solution, resulted in $85 \%$ quenching of the QD emission within 1.5 hours. The close proximity is achieved due to the affinity of the 3'-DNA end to the 5'end of its complimentary DNA strand, thus bringing the Au in close contact with the QD. A full recovery of QD emission is obtained within 1 hour when a 10-fold excess of its complimentary DNA strand, without gold attached, is added to the solution.

Apart from using complimentary DNA sequences to facilitate QD/gold FRET coupling, the close proximity and FRET coupling can be achieved by binding streptavidin coated QDs with biotinylated gold nanoparticles [75]. It is also noted that 620nm emission QDs were FRET coupled to Au nanoparticles by this group, compared to the $540 \mathrm{~nm}$ emission QDs used in the aforementioned DNA-binding study. Therefore a wide range of QDs can be effectively FRET coupled with gold nanoparticles. In addition, the quenching effects of gold nanoparticles on immobilized QDs were also studied. The schematic for QD immobilization is shown in Figure 20.

The glass slide was initially rinsed with $1 \mathrm{M}$ aqueous ammonia and washed with water. The glass slide was incubated for 12 hours in $1 \mathrm{mg} / \mathrm{mL}$ bovine serum albumin (BSA)biotin, dissolved in $20 \mathrm{mM}$ phosphate buffer at $\mathrm{pH} 7$ [76]. Following incubation, the glass slide was rinsed with water to remove any unabsorbed BSA-biotin, resulting in a monolayer of BSA-biotin absorbed onto the glass surface. The streptavidin was added to the system by putting a drop of $1 \mathrm{mg} / \mathrm{mL}$ streptavidin, dissolved in $20 \mathrm{mM}$ phosphate buffer at $\mathrm{pH} 7$, on the functionalized glass slide and incubating for 1 hour. Again the glass slide was rinsed to remove any unattached streptavidin. Biotin was attached to the 3'-end of the QD-DNA, so the incubation of $10 \mu \mathrm{L}$ of $0.156 \mu \mathrm{M}$ biotinylated QD-DNA to 
the glass slide $\left(1 \mathrm{~cm}^{2}\right.$ area) for 15 minutes resulted in immobilization and orientation of the QD-DNA [74]. The fluorescent quenching of the QDs resulting from the addition of its complimentary Au-DNA is imaged in Figure 21.

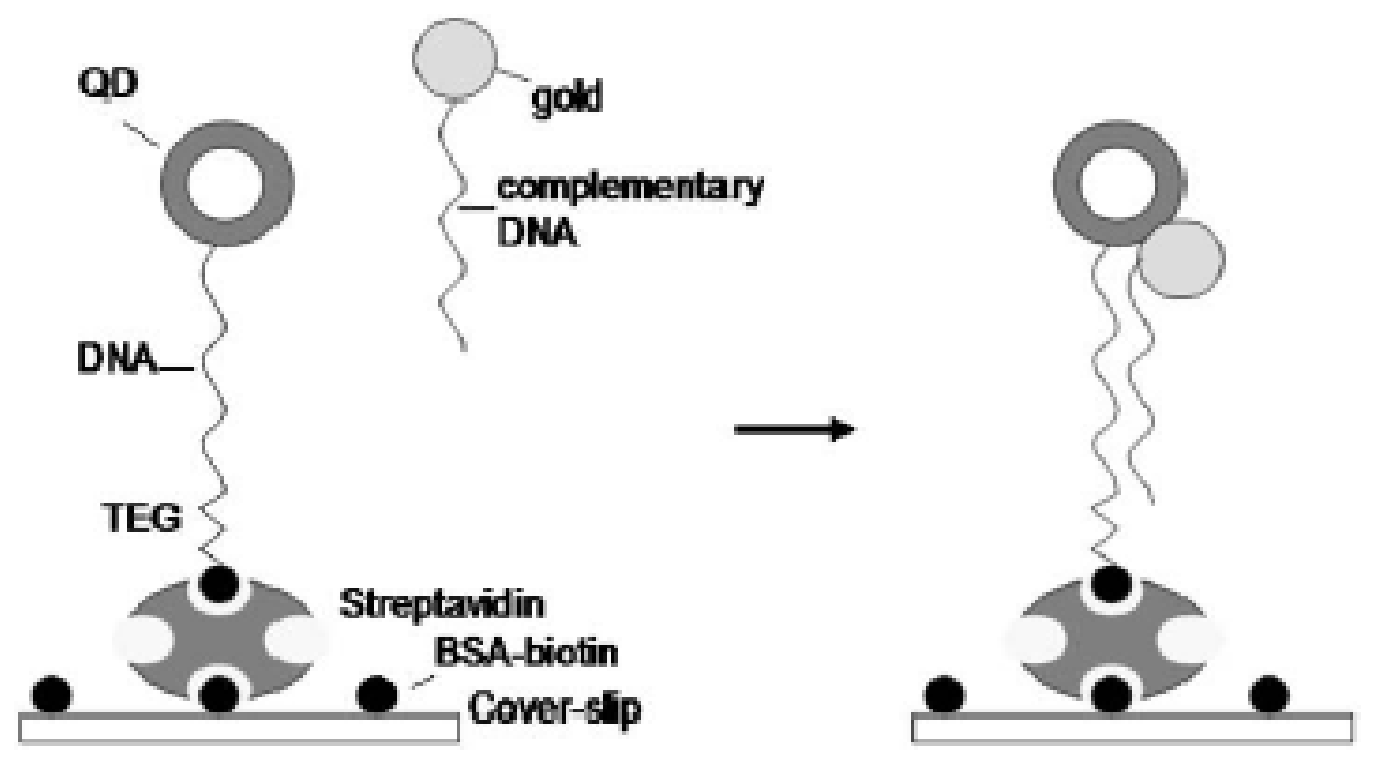

Figure 20. Schematic of hybridization of Au-DNA with immobilized QD-DNA-biotin [74]. Dyadyusha, L., et al., Quenching of CdSe quantum dot emission, a new approach for biosensing. Chem. Commun., 2005: p. 3201-3203 - Reproduced by permission of The Royal Society of Chemistry. Permission can be found in Appendix A.7.
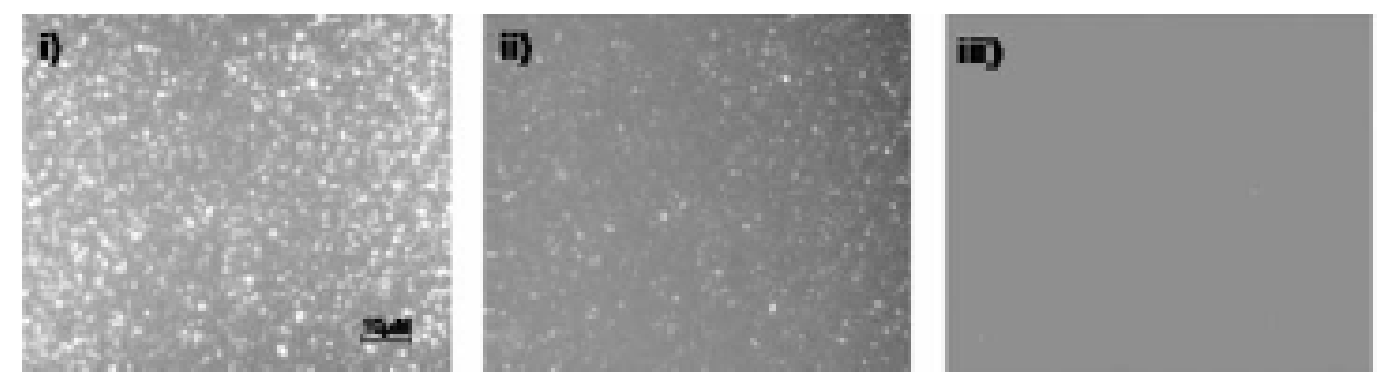

Figure 21. Epiflourescence image of i) QD-DNA-biotin associated on streptavidin functionalized glass slide, ii) after addition of Au-DNA (5 min), iii) after addition of Au-DNA (15 min) [74]. Dyadyusha, L., et al., Quenching of CdSe quantum dot emission, a new approach for biosensing. Chem. Commun., 2005: p. 3201-3203 - Reproduced by permission of The Royal Society of Chemistry. Permission can be found in Appendix A.7. 
It should be noted, that the FRET quenching of the QDs occurred at a much faster rate, 15 minutes compared to 90 minutes, than in the aqueous suspension study.

In summary, previous studies have shown that CdSe QDs are extremely robust for engineering applications, easily accommodate surface functionalization/modification, and are capable of serving as a FRET acceptor with a wide array of both organic and inorganic particles. The ability to tune QDs to emit at 570nm makes them a viable nanoscale light activation source for bR-based sensors. Additionally the ability to form a $\mathrm{bR} / \mathrm{QD}$ FRET coupling system has the potential for altering the bR electrical output based upon the degree of FRET coupling. This phenomenon could serve as the modulation method for target molecule detection.

\subsection{IONIC-SELF ASSEMBLED MONOLAYERS}

The Ionic-Self Assembled Monolayer (I-SAM) technique provides a means of constructing multi-layered monolayer films of oppositely charged materials. The I-SAM technique is referenced by several synonymous names in the literature, including electrostatic layer-by-layer assembly and electrostatic self assembly [77-80].

\subsection{1. bR Ionic-Self Assembled Monolayer Films}

For applications with PM, the I-SAM technique has proven to be a quick and efficient method for creating highly-oriented PM monolayer films [81-84]. The bR molecule can serve as a polyanion in the I-SAM layering structure due to its increased negative charge at elevated $\mathrm{pH}$ levels. The isoelectric point of $\mathrm{bR}$ is around $\mathrm{pH} 5.2[85,86]$. When brought above this $\mathrm{pH}$ level, deprotonation of amino acids in the bR molecule occurs resulting in a net negative charge. For a high density PM I-SAM film, it is important to have a strong negative charge to allow maximal absorption to the polycation layer. Studies have shown that PM suspended at pH 9.4 has over twice the absorptive capability 
as PM suspended at $\mathrm{pH}$ 5.5, with a linear relationship observed between these $\mathrm{pH}$ values [82]. Suspending $\mathrm{PM}$ at $\mathrm{pH}$ levels higher than $\mathrm{pH} 9.4$ is difficult due to the fact that $\mathrm{bR}$ bleaches and the retinal is released around $\mathrm{pH} 10$ and higher, thus destroying the $\mathrm{bR}$ molecule's photoelectric activity.

A typical layering protocol involves using polydimethyldiallyammonium chloride (PDAC) as the polycation and PM as the polyanion. Figure 22 displays a schematic of the bR/PDAC I-SAM layering process. Studies have shown that bR I-SAM films have a higher degree of protein orientation than electrophoretically deposited bR films, thus improving the films photoelectric properties.

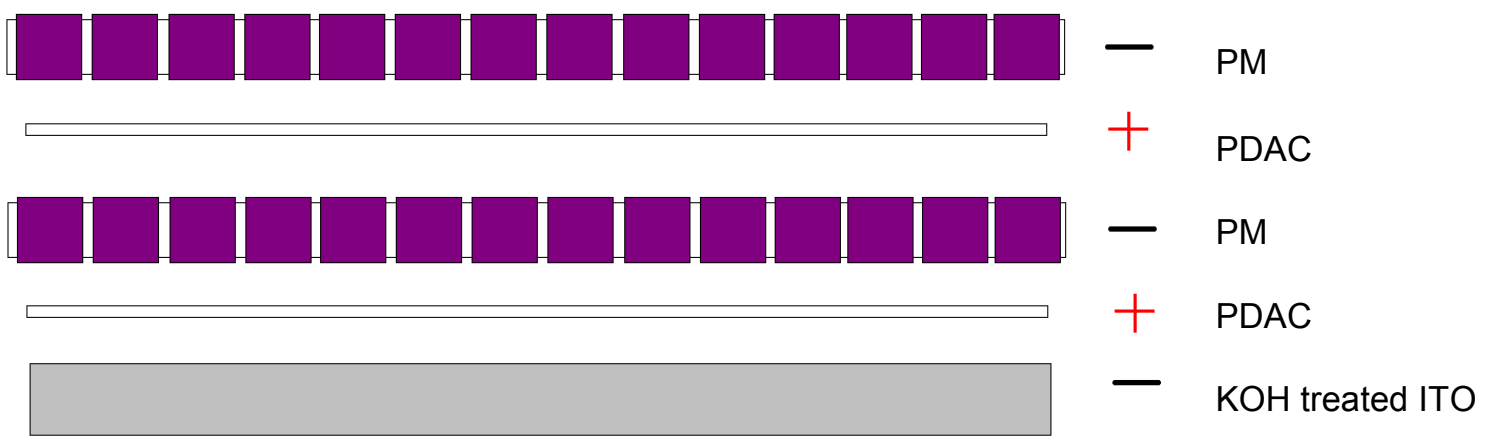

Figure 22. Schematic of bilayer I-SAM films constructed on negatively charged ITO with PDAC serving as the polycation and PM serving as the polyanion.

Alternate layering of PM/PDAC has been used to construct multi-layered films up to 14 bilayers thick [82]. The optimal thickness to achieve maximal bR photocurrent, however, was determined to be approximately 8 bilayers for wild-type PM as shown in Figure 23 [81]. All the measurements of the bR photoelectric activity in these I-SAM films have been performed in a $\mathrm{KCl}$ electrolyte solution. This suggests that the photocurrent being measured is due to the change in proton concentration at the ITO electrode surface, and thus the closest PM layers have the largest effect on the photocurrent output [81]. No specific applications of PM thin films using the I-SAM technique have been reported to date. 


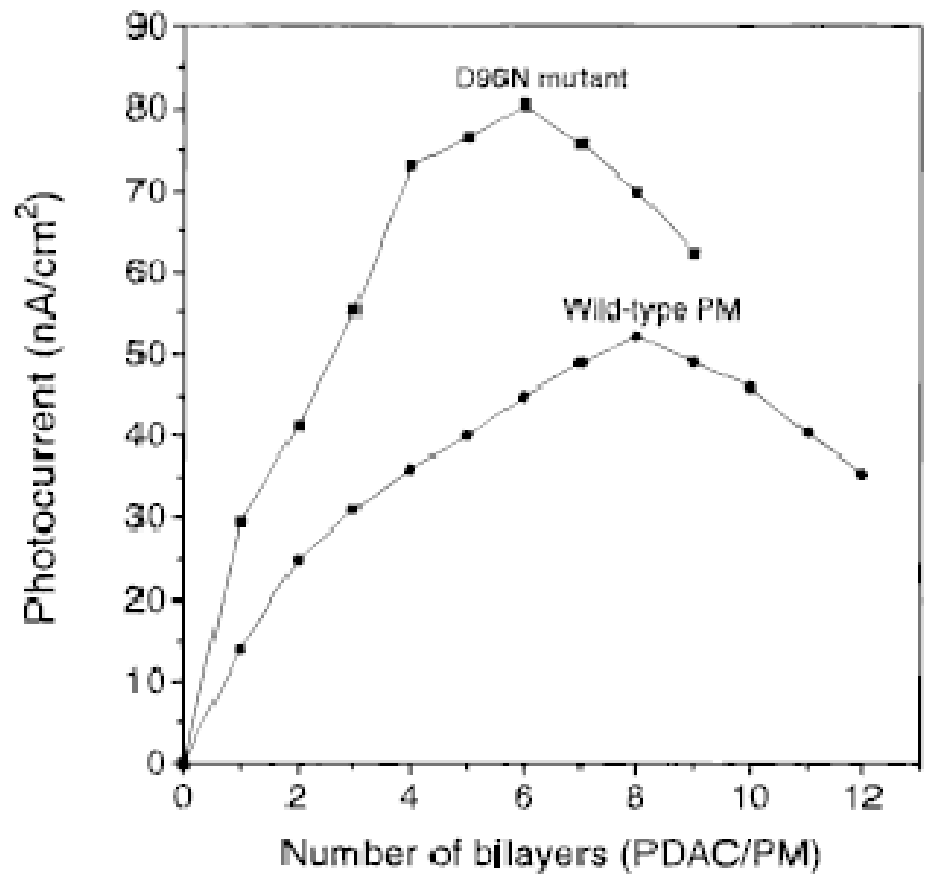

Figure 23. Photocurrent generated from multi-layered PM films produced with the I-SAM technique. Maximal output is achieved between 6 to 8 bilayers for the two different types of PM tested $[80]$.

\subsubsection{QD Ionic-Self Assembled Monolayer Films}

The application of QDs in monolayer films has been widely studied. This has lead to the optimization of several techniques to accomplish QD monolayer deposition, including Langmuir-Blodgett [87-89], spin coating techniques [90], and I-SAM techniques [91-93]. For this work the I-SAM technique is the ideal method for QD monolayer deposition for its ease of application and ability to efficiently integrate other I-SAM compatible materials into the monolayer film.

To apply QDs in an electrostatic layer-by-layer fashion, they must be made water soluble and given either a positive or negative charge. One popular method of making QDs water soluble also effectively gives the QDs a negative charge. A majority of assynthesized $\mathrm{CdSe} / \mathrm{ZnS}$ QDs possess a TOPO surface functional group which must be 
replaced to make the QDs water soluble. A popular method to accomplish this is to perform a ligand-exchange procedure which replaces the TOPO group with mercaptoacetic acid (MAA) [94], which is also known as thio-glycolic acid (TGA). Along with making the QDs water soluble, the carboxyl groups on the surface TGA molecules also allow for the creation of a net negative charge on the QD nanocrystal. At elevated $\mathrm{pH}$ levels, approximately $\mathrm{pH} 8-10$, the carboxyl group can be de-protonated and yield a negative charge of approximately $-30 \mathrm{mV}$ [95]. As shown in Figure 24, the charge of the QD can then be easily altered through the binding of a charged polymeric material to the QD surface. Specifically, polyallyl amine (PAA) has been used to create a positively charged QD upwards of $+30 \mathrm{mV}$ and polyvinyl sulfonic acid (PVSA) has been employed to increase the QD negativity to $-70 \mathrm{mV}$ [95].

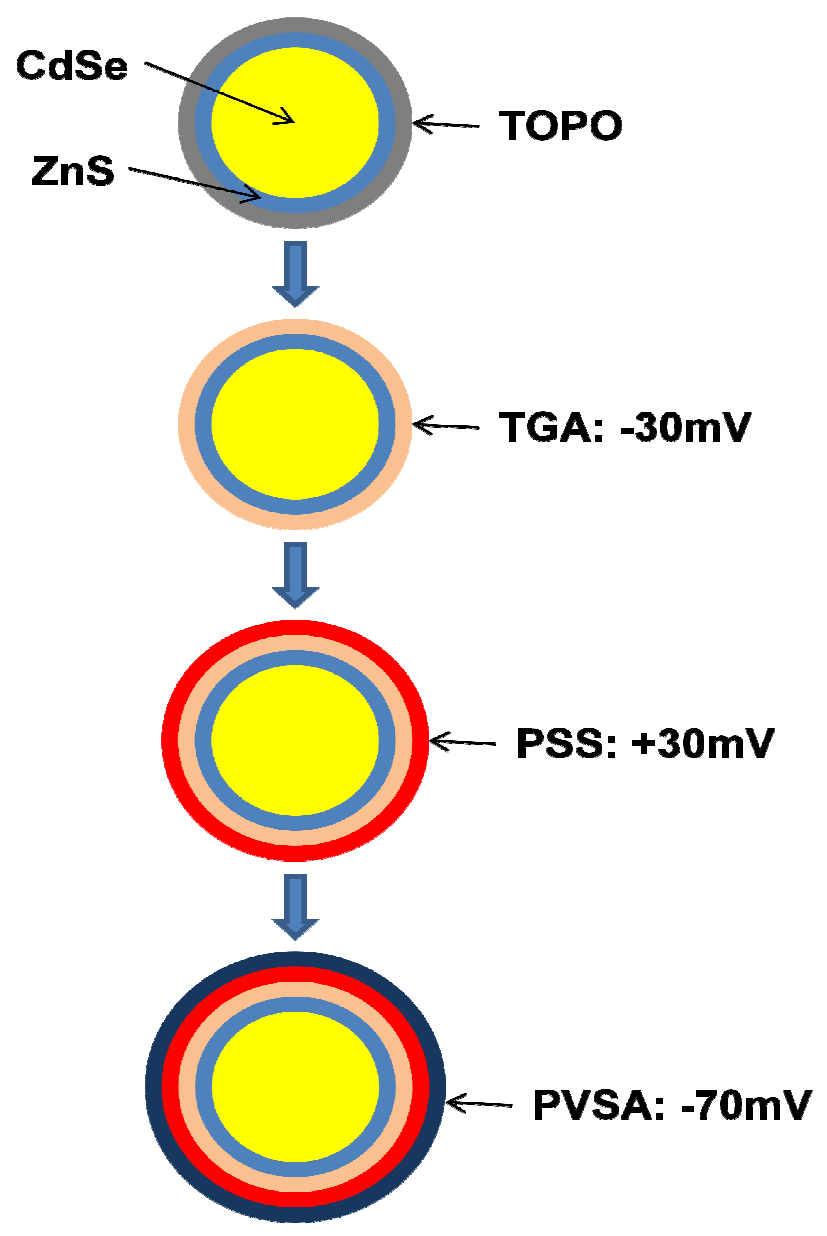

Figure 24. QD surface modifications for I-SAM applications. 
With the desired electrostatic charge achieved on the QD, it can then be deposited as an ISAM material. As with the I-SAM deposition of bacteriorhodopsin, an oppositely charged polyionic material such as PDAC $(+)$ or PSS (-) can be used as the spacer material for multi-layered deposition [93]. Since it is possible to create QDs of either positive or negative charge, however, it is possible to bypass the use of a polyionic spacer layer through the use of alternately charged QDs [95]. Similarly, when creating a multilayered hybrid film it is feasible that the QD can be tailored to the opposite charge of the other I-SAM material.

A published application of QD-hybrid systems has been demonstrated in the field of biosensors [92]. This work used TGA-capped QDs as the negative electrostatic material in conjunction with organophosphorus hydrolase (OPH) [59] and chitosan as the positive charged nanomaterial. By creating the thin film structure they were able to create a biosensing system capable of detecting paraoxon.

The main sensing mechanism of the described system is to monitor organophosphorus hydrolase [59] break-down of paraoxin via UV-VIS techniques. Their studies revealed, however, that the photoluminescence of the QDs could also be used for paraoxin detection. It was found that as paraoxin levels increased, QD photoluminescence decreased. It is proposed that the OPH-paraoxin reaction results in conformational changes in the OPH film that ultimately affects the passivation level of the QDs. Altering the extent of water molecules interacting with the QDs is known to introduce surface traps and ultimately reduce QD emission intensity [87]. In addition it is proposed that the QD electron-hole recombination process is interrupted by the $\mathrm{OPH}$, ultimately resulting in an energy coupled system. The ability of QD-QD monolayer FRET coupled systems has been previously demonstrated [96], however the ability for QDs to potentially FRET couple to other nanomaterials in I-SAM films is of interest in the proposed bR-QD sensing system. 


\section{Chapter 2. Protocol for bR Production}

For engineered applications, bR is processed into membrane patches, known as purple membrane (PM). Purple membrane is simply a large cell membrane patch, on average $500 \mathrm{~nm}$ in diameter, which is composed of multiple bR molecules and their associated lipids. It is called PM due to its distinct purple color, which is due to the absorption properties of the $\mathrm{bR}$ molecules. The process of preparing and purifying bacteriorhodopsin for engineered applications has been extensively described [20] and has been utilized with slight modifications for the stated production protocol. Purchasing purified bR from a supplier, such as Sigma Aldrich, would cost upwards of $\$ 400 / \mathrm{mg}$. Compared to in house costs of $\$ 1.67 / \mathrm{mg}$ (at estimates of $\$ 84.26$ per $10 \mathrm{~L}$ batch, resulting in $70 \mathrm{mg}$ bR produced), the need to create a $\mathrm{bR}$ production process in-house was apparent. The entire bR production protocol is outlined in Figure 25 and elaborated upon below. This procedure utilizes the S9-P strain, a bR overproducer, which was estimated to produce 50-500mg of bR per 10L batch. The H. halobium S9-P strain was obtained from John Spudich at the University of Texas Medical School. Additional information on the bR production protocol is available in Chris Anton's $\mathrm{PhD}$ thesis [97].

The media used to grow the H. halobium is composed of the materials listed in Table 2, added in that order to distilled-deionized water. The final solution is adjusted to $\mathrm{pH} 7.2$ with $5 \mathrm{~N} \mathrm{NaOH}$. Prior to inoculation the solution is sterilized utilizing an autoclave and allowed to cool back to room temperature. The final media will have a distinct smell due to the bacteriological peptone and will be a yellowish-brown color. 


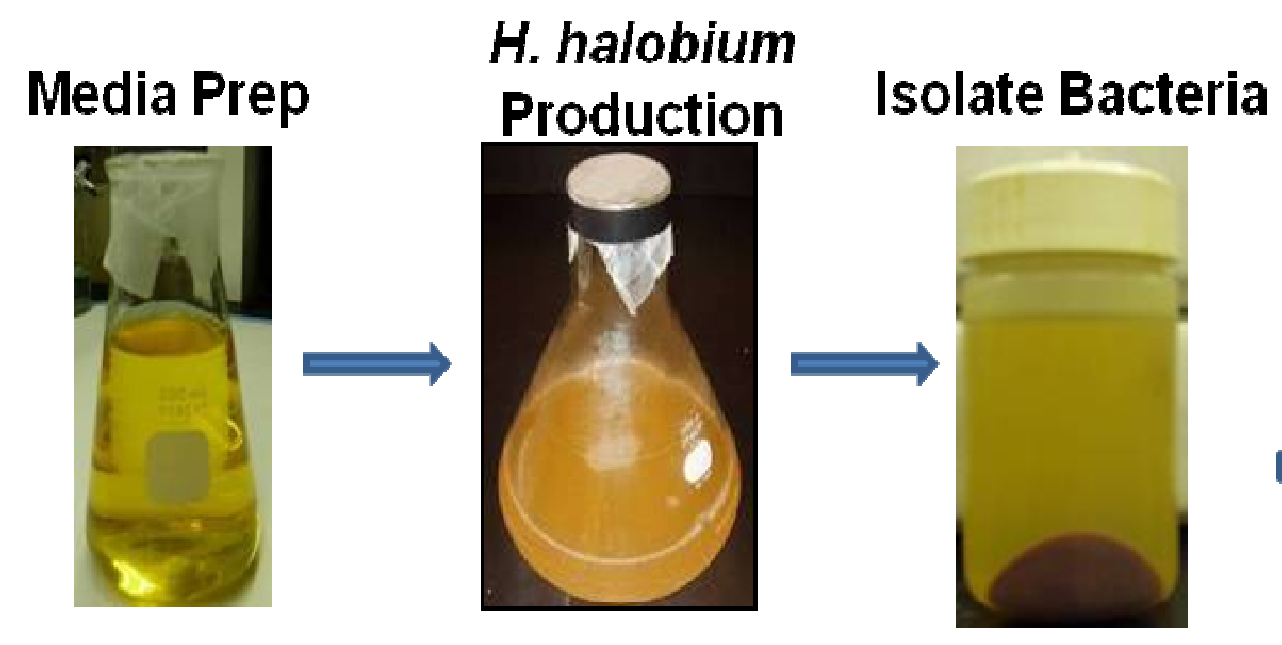

Remove Fine Remove Large Lyse Cells/ Destroy Cellular Debris Cellular Debris DNA Chains

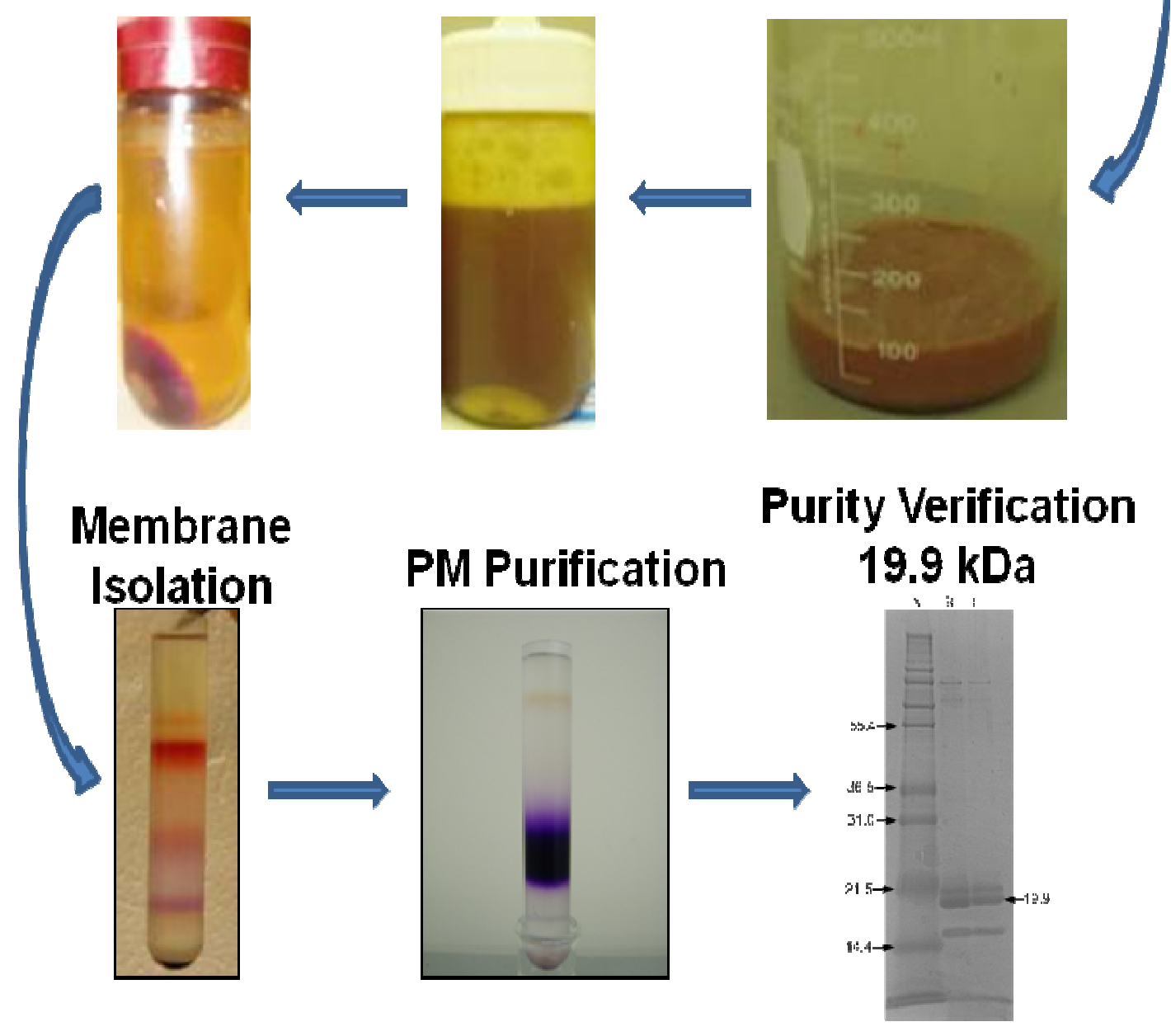

Figure 25. Outline of H. halobium production and PM isolation/purification. 
Table 1. Materials for H. halobium media. Chemicals in order of addition.

\begin{tabular}{|l|c|c|c|}
\hline Reagent & Concentration & Per 1 Liter & Per 10 Liters \\
\hline $\mathrm{NaCl}$ & $4.28 \mathrm{M}$ & $250 \mathrm{~g}$ & $2.5 \mathrm{~kg}$ \\
\hline $\mathrm{MgSO}{ }_{4}$ (anhydrous) & $81.1 \mathrm{mM}$ & $9.77 \mathrm{~g}$ & $97.7 \mathrm{~g}$ \\
\hline $\mathrm{KCl}$ & $26.8 \mathrm{mM}$ & $2 \mathrm{~g}$ & $20 \mathrm{~g}$ \\
\hline $\mathrm{NH}_{4} \mathrm{Cl}$ & $93.5 \mathrm{mM}$ & $5 \mathrm{~g}$ & $50 \mathrm{~g}$ \\
\hline Sodium Citrate. $2 \mathrm{H}_{2} \mathrm{O}$ & $10.2 \mathrm{mM}$ & $3 \mathrm{~g}$ & $30 \mathrm{~g}$ \\
\hline $\mathrm{Glycerol}$ & $137 \mathrm{mM}$ & $1 \mathrm{ml}$ & $10 \mathrm{ml}$ \\
\hline $\mathrm{KH}_{2} \mathrm{PO}_{4}$ & $0.735 \mathrm{mM}$ & $0.1 \mathrm{~g}$ & $1 \mathrm{~g}$ \\
\hline $\mathrm{CaCl}_{2}$ (anhydrous) & $1.4 \mathrm{mM}$ & $0.2 \mathrm{~g}$ & $2 \mathrm{~g}$ \\
\hline $\mathrm{Bacteriological} \mathrm{Peptone}$ & - & $10 \mathrm{~g}$ & $100 \mathrm{~g}$ \\
\hline
\end{tabular}

The collected media was centrifuged in $400 \mathrm{ml}$ increments in 2-4 different tubes, measured equal by weight. A Beckman centrifuge J-14 rotor was used and all bottles were simultaneously spun for 15 minutes at 8,000 RPM. A reddish/purple pellet formed in the bottom edge of the tubes, which contains the H. halobium cells. The supernatant was collected and discarded from the tubes. More collected media is added to both tubes, the pellet remaining on the bottom, until the tubes had equal weight for the next centrifuge run. This process continued until all of the collected media was centrifuged. As expected, the pellet on the bottom of each tube continuously grew throughout this process.

After all the media had been centrifuged, the pellet was scraped out of the bottles and placed in a clean glass beaker. Deionized water was then added to the beakers to resuspend the pellet. The addition of water caused cell lysis to occur and the suspension became very viscous due to the uncoiling of DNA. To reduce this viscosity, DNASE I was added to the suspension at $25 \mu \mathrm{g} / \mathrm{ml}$ to cleave the DNA chains. The DNASE I was allowed to sit for 24 hours to fully destroy DNA chains. It is possible that not all cells in the suspension had lysed at this point, so more deionized water was added. If the suspension once again gained viscosity, then more DNASE I was added. 
After all of the cells were lysed and the DNA chains had broken, the unwanted cellular debris was removed. The suspension was centrifuged in a JA-20 rotor for 15 minutes at 5,000 RPM. The pellet contained the unwanted cellular debris, so the supernatant, which contained the purple membrane, was collected. The supernatant was then recentrifuged in a Ti-45 rotor for 30 minutes at 35,000 RPM. This time the pellet contained the desired purple membrane and the supernatant contained unwanted material. The supernatant was removed and the pellet was resuspended in a small amount of deionized water. This centrifuge step was repeated until the supernatant was clear.

Sucrose concentrations of $20 \%, 36 \%, 40 \%$, and $46 \%$ were created. The sucrose gradients and bR membrane were then carefully injected into centrifuge tube as depicted in Figure 26. Two vials were created and they were placed in a Beckman ultracentrifuge utilizing a SW-41TI swinging bucket rotor. The centrifuge was run at 20,000 RPM for 18 hours at $4^{\circ} \mathrm{C}$. The densities of the fragment particles control the degree of particle migration through the varying sucrose densities during centrifugation. Upon removal of tubes from centrifuge, obvious gradients were present as is displayed in Figure 26.

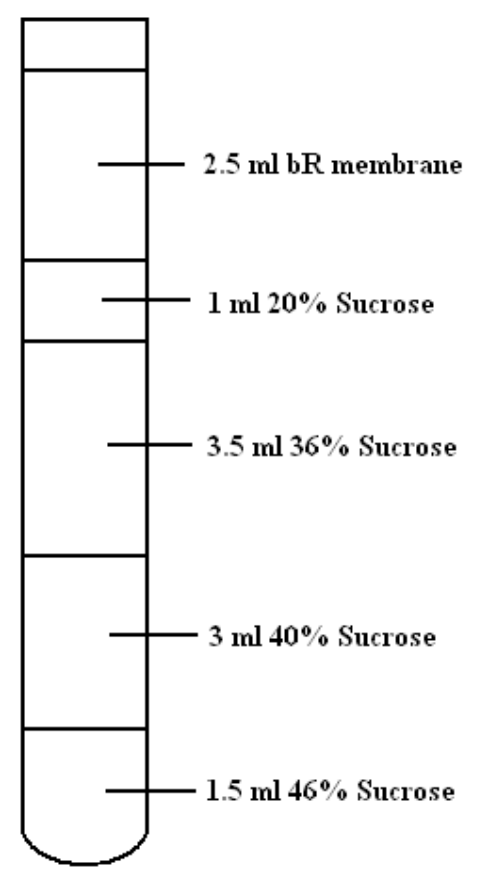

Figure 26. Sucrose gradient percentages for bR purification. 
The purple gradient was removed and placed in another plastic vial where deionized water was added. The purpose of adding water was to dilute the sucrose so a purple membrane pellet would form upon the next centrifugation step. Two even vials were created and centrifuged at 40,000 RPM for 1 hour at $4{ }^{\circ} \mathrm{C}$. A Ti-70.1 fixed angle rotor was used for this step. The pellet was resuspended in a small amount of deionized water $(\sim 3 \mathrm{ml})$. The average PM fragment size was analyzed with a particle size analyzer and determined to be $497.6 \mathrm{~nm}$ as displayed in Figure 27.

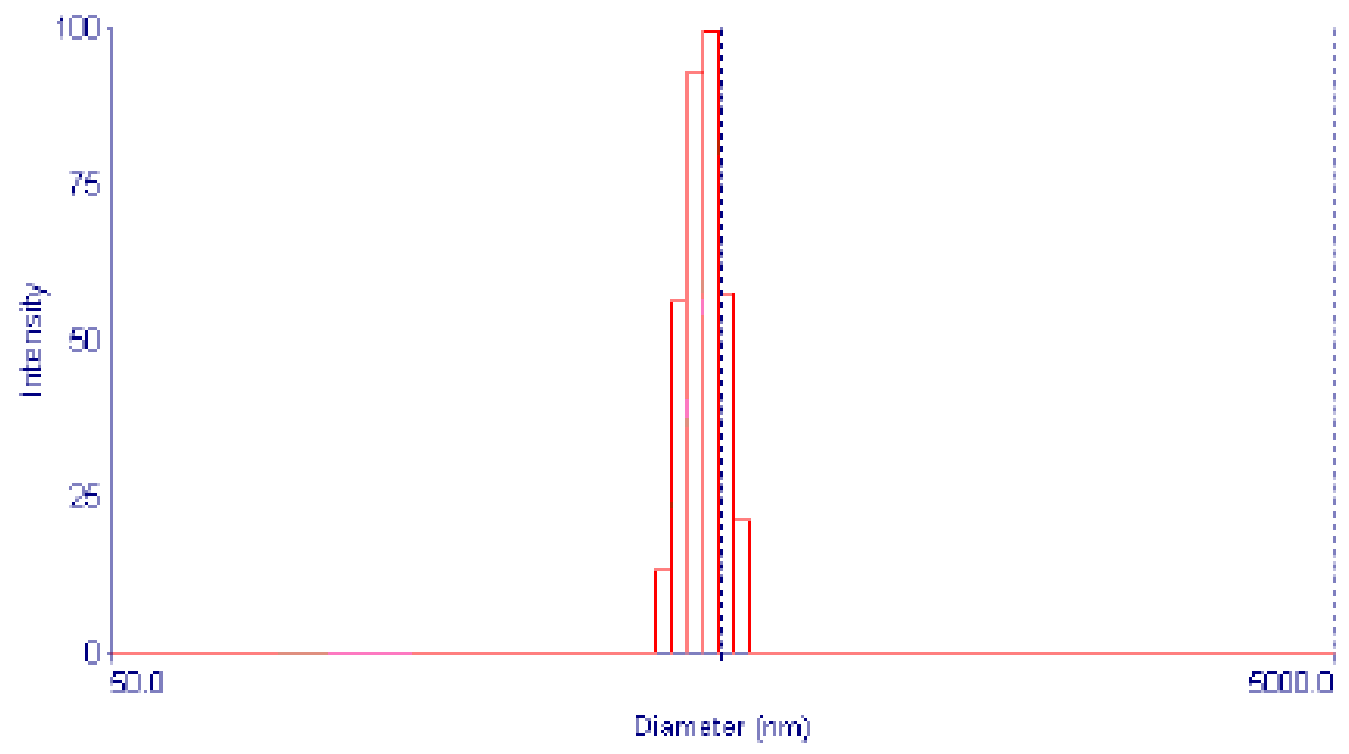

Figure 27. Particle size analysis of PM fragments showing an average fragment diameter of $497.6 \mathrm{~nm}$ with a size range of $399.3 \mathrm{~nm}-\mathbf{5 3 6 . 4} \mathrm{nm}$.

The purity of the resulting bR preparation was evaluated by sodium dodecyl sulfatepolyacrylamide gel electrophoresis (SDS-PAGE) on a $12 \%$ Bis-Tris gel. It has been shown that the relative molecular mass [98] of bR in SDS decreases to 19.9kDa [99]. The final purified PM was aliquoted at approximately $2 \mathrm{mg} / \mathrm{ml}$ and stored at $-80^{\circ} \mathrm{C}$ until use. 


\section{Chapter 3. bR-Based Electrodes}

Optically sensitive protein electrodes utilizing bacteriorhodopsin (bR) have been produced and shown to be electrically active with consistent photovoltage and photocurrent outputs. The following methods were used in the electrode construction and measurement process.

\subsection{ELECTROPHORETIC DEPOSITION LAYERING/ORIENTATION}

Due to the specific proton pumping direction of $b R$, the thin $b R$ films used to make the photosensitive electrodes must be highly oriented. This allows a maximum number of protons to be shifted in the same direction and yields a higher electrical voltage. The high degree of protein orientation for these studies was obtained through the well documented technique of electrophoretic sedimentation.

To create the bR-based photosensitive electrodes, a fixture was constructed to hold the two electrodes apart, with separation distances of $2 \mathrm{~mm}, 1 \mathrm{~mm}, 0.5 \mathrm{~mm}, 0.25 \mathrm{~mm}$, or $0.1 \mathrm{~mm}$. The bottom electrode (positive electrode) was a glass slide with a thin layer of optically transparent and electrically conductive indium tin oxide [22] sputtered on top. The dimensions of the electrode were $5 \mathrm{~mm} \times 10 \mathrm{~mm}$ and the ITO had a sheet resistance of $5-10 \Omega /$ square. A $30 \mu 1$ suspension of $15 \mathrm{mg} / \mathrm{mL}$ PM in ddI water was pipetted on top of the ITO and a brass electrode (negative electrode) was placed on top with $1 \mathrm{~mm}$ spacing between the electrodes. An electric field of $40 \mathrm{~V} / \mathrm{cm}$ was placed through the electrodes for 1 minute, as depicted in Figure 28. It was possible to visually observe the PM deposit out of its distilled/ deionized water suspension and attach to the positive electrode while the electric field was applied. 


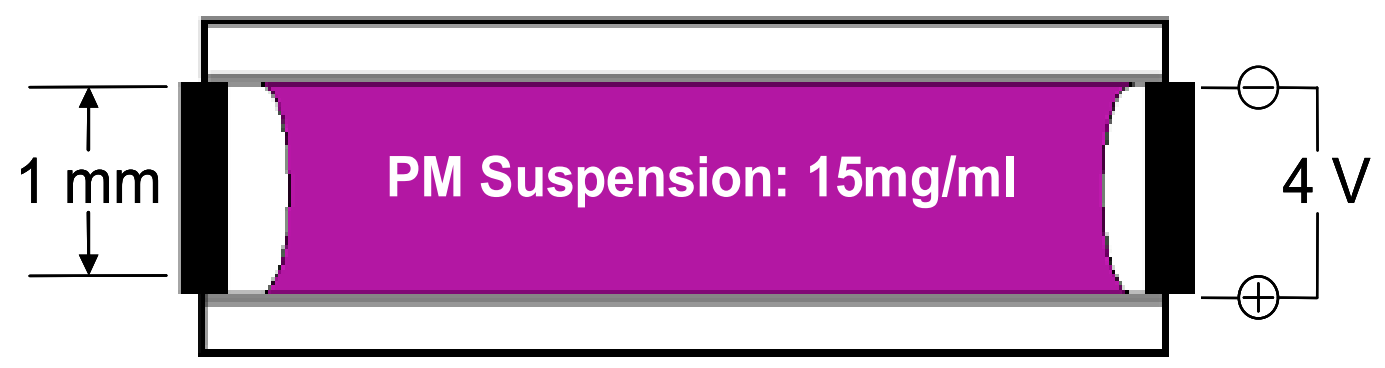

-Apply Voltage for 1 minute

-Cytoplasmic side attaches to

positive electrode

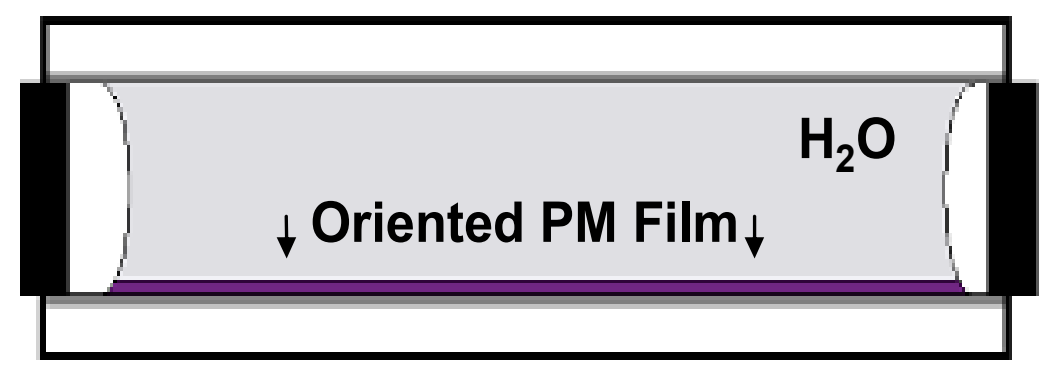

-Remove Water, Rinse, Dry for $24 \mathrm{hr}$ at $54 \%$ Humidity.

Figure 28. PM orientation thru electrophoretic sedimentation

Following the 1 minute exposure to the electric field, the top brass electrode was removed and the excess water was pipetted off the oriented PM. The ITO electrode with attached PM was then rinsed for 10 seconds in a beaker of ddI water to remove any weakly attached PM. The ITO electrode was then stored in a humidity chamber for 24 hours to dry. Humidity in the chamber was maintained at $\sim 52 \%$ through the use of a magnesium nitrate salt solution. The dimensions of the dried bR film were approximately $5 \mathrm{~mm} \times 5 \mathrm{~mm}$ with a thickness of approximately $20 \mu \mathrm{m}$ measured with an inteferometric microscope. After drying another ITO electrode of similar dimensions was placed on top of the oriented bR film as depicted in Figure 29. 


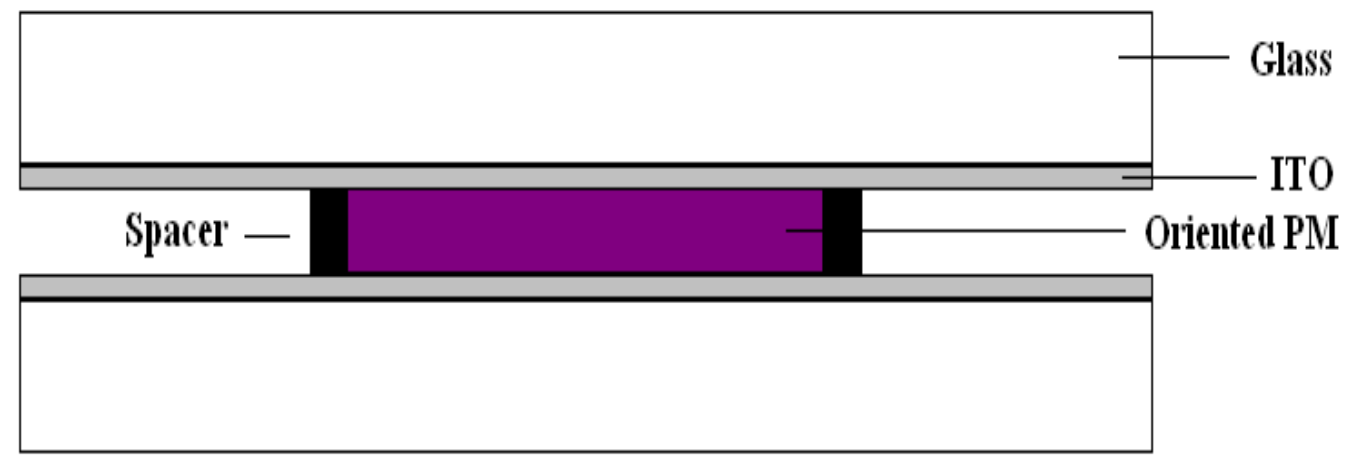

Figure 29. Final bR-based electrode

A thin spacer could be used to help prevent contact between the two ITO slides, but was not necessary in this study. The two electrodes were then permanently sealed together with adhesive to prevent any movement between the electrodes and to prevent further drying of the bR film. A wire was connected to each ITO electrode which was later connected to a signal amplifcation circuit.

\subsection{Electrical Measurement SETUP}

The constructed bR electrode was wired directly into a home-built, non-inverting operational amplifier [24]. The amplification factor of the non-inverting op-amp was adjusted to 10X by modifying the resistor values. The schematic is shown in Figure 30.

The ITO electrode with the cytoplasmic side of the bR attached to it was set to ground (-) and the opposing ITO electrode was attached to the op-amp (+). Both the op-amp and bR electrode were placed in a Faraday cage. The non-inverting op-amp was powered by an HP 6237A triple output voltage supply. The signal from the op-amp was passed through a low frequency noise filter (Krohn-Hite Model 3364) to remove all noise between $30 \mathrm{~Hz}$ and $120 \mathrm{~Hz}$. From the filter, the conditioned signal was input to a $60 \mathrm{MHz}, 200 \mathrm{MSa} / \mathrm{s}$ oscilloscope (Agilent 54621D) for display. 


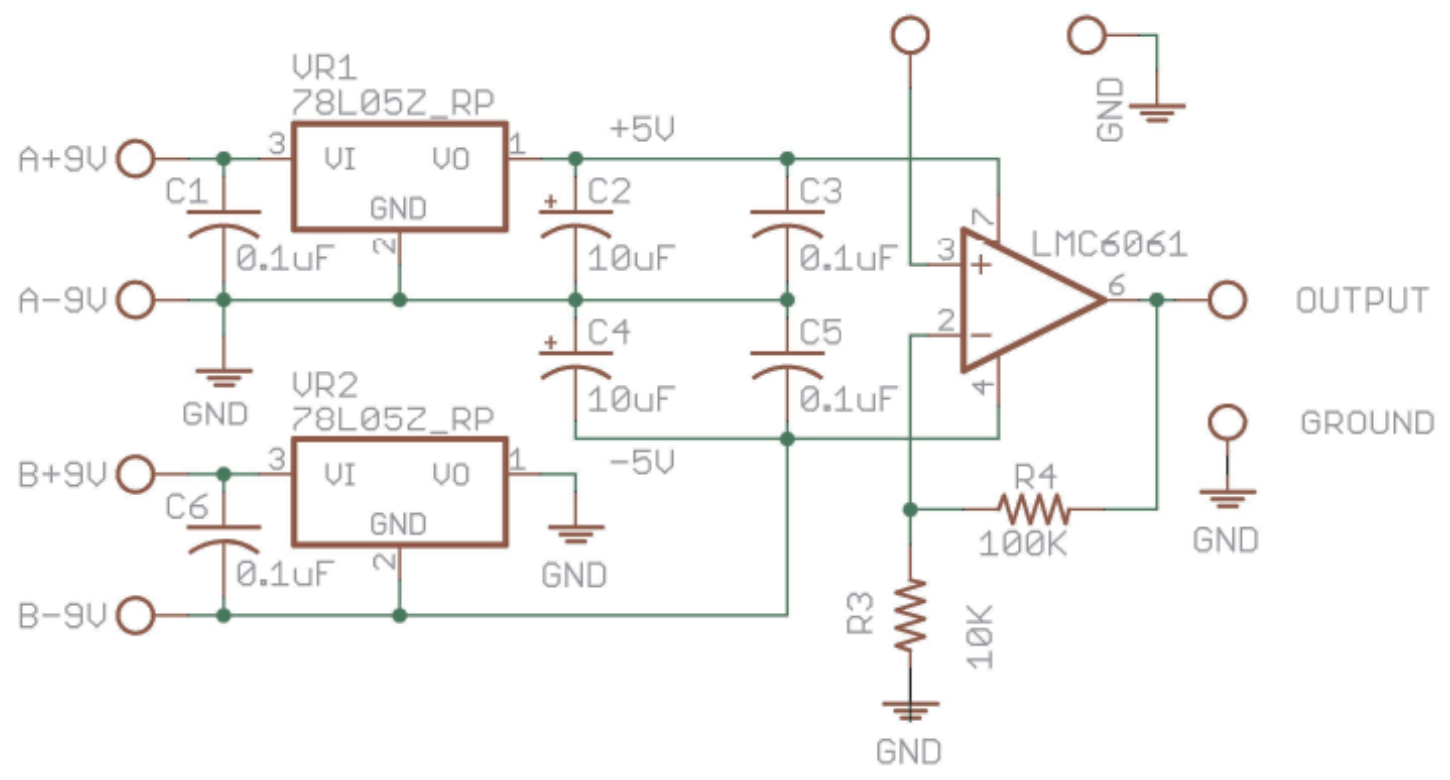

Figure 30. Inverting op-amp design [100].

The electrical amplification circuitry has been further developed into chip-form and is further discussed in Karl Walczak's dissertation [100]. This amplifier can be easily developed for selected amplification, from $1 \mathrm{x}$ to over 10x.

\subsection{PhOtoelectric ACTIVITY OF Electrodeposited PM FiLMS}

In a dried bR electrode, it has been shown that the proton doesn't actually pump through the bR molecule, but rather shifts within it upon light absorption [29]. Due to dehydration it is not possible for bR to achieve all of the intermediate steps, as shown in Figure 31. 


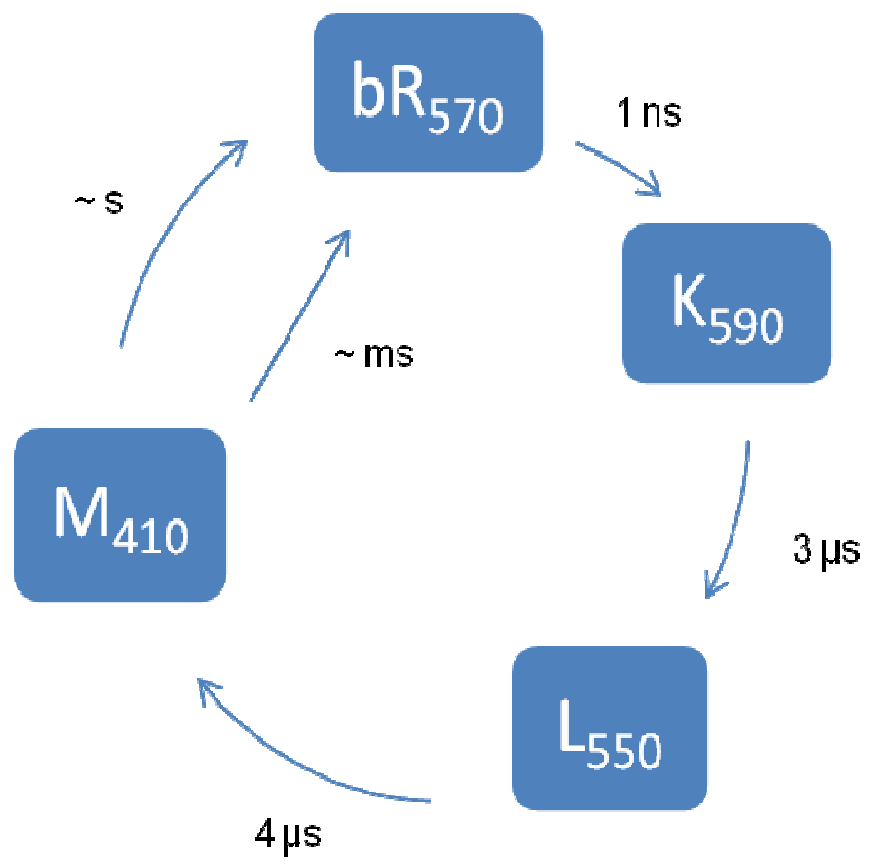

Figure 31. Photocyle intermediate of dried film bR after photo-illumination.

Although no actual proton is pumped out of the dehydrated bR molecule upon photoillumination, a strong electrical response still results from the protons shifting in the bR molecules. In this setup the PM is oriented on an ITO slide via the electrodeposition method and a separate ITO electrode is placed on top of the PM surface to serve as the counter electrode. An example of the resulting photovoltaic response of a prepared sample in response to illumination with an incandescent light source is shown in Figure 32. From Figure 32, the characteristic sharp voltage spike upon illumination followed by a gradual decay is noted. The transient behavior is due to a combination of the $b R$ photovoltaic response and the measuring instrumentation. The amplified voltage spike in this sample peaks at $16 \mathrm{mV}$ and was visible for $\sim 1.5$ seconds. The voltage spike is due to the movement of the charge in the PM film resulting from illumination. Since there is no actual proton pumping in dried PM films, the proton is held in its shifted location until the light is subsequently turned off. Upon turning off the light illumination, the protons shift back to their original location. This final charge movement of the protons moving back to their original location resulted in the negative spike. The signal shown is the 
characteristic bR signal when the measuring oscilloscope is setup in AC-coupled mode. Shown in Figure 33 is a steady-state bR photovoltage resulting when the oscilloscope is setup in DC-coupled mode.

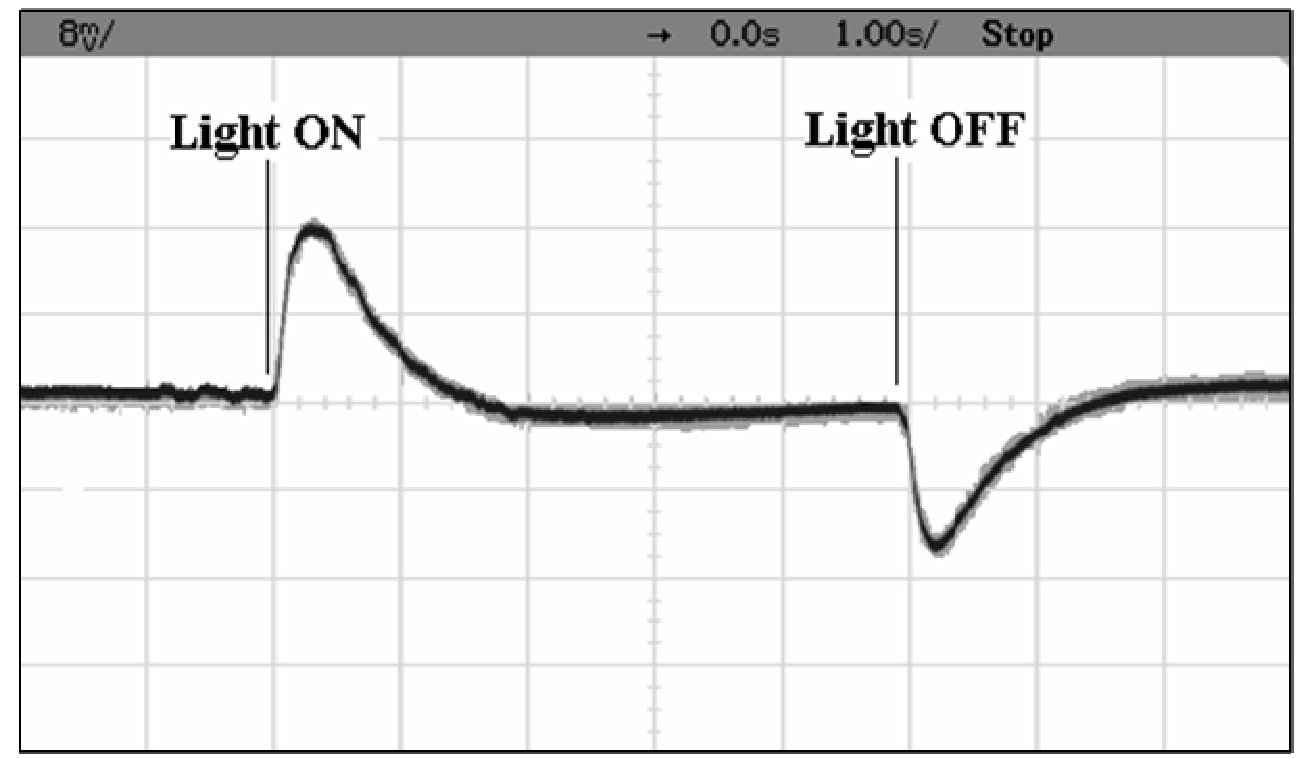

Figure 32. bR Photoelectric response to incadescent light illumination with AC-coupling $(8 \mathrm{mV} /$ vertical division, $1 \mathrm{sec} / \mathrm{horizontal}$ division)

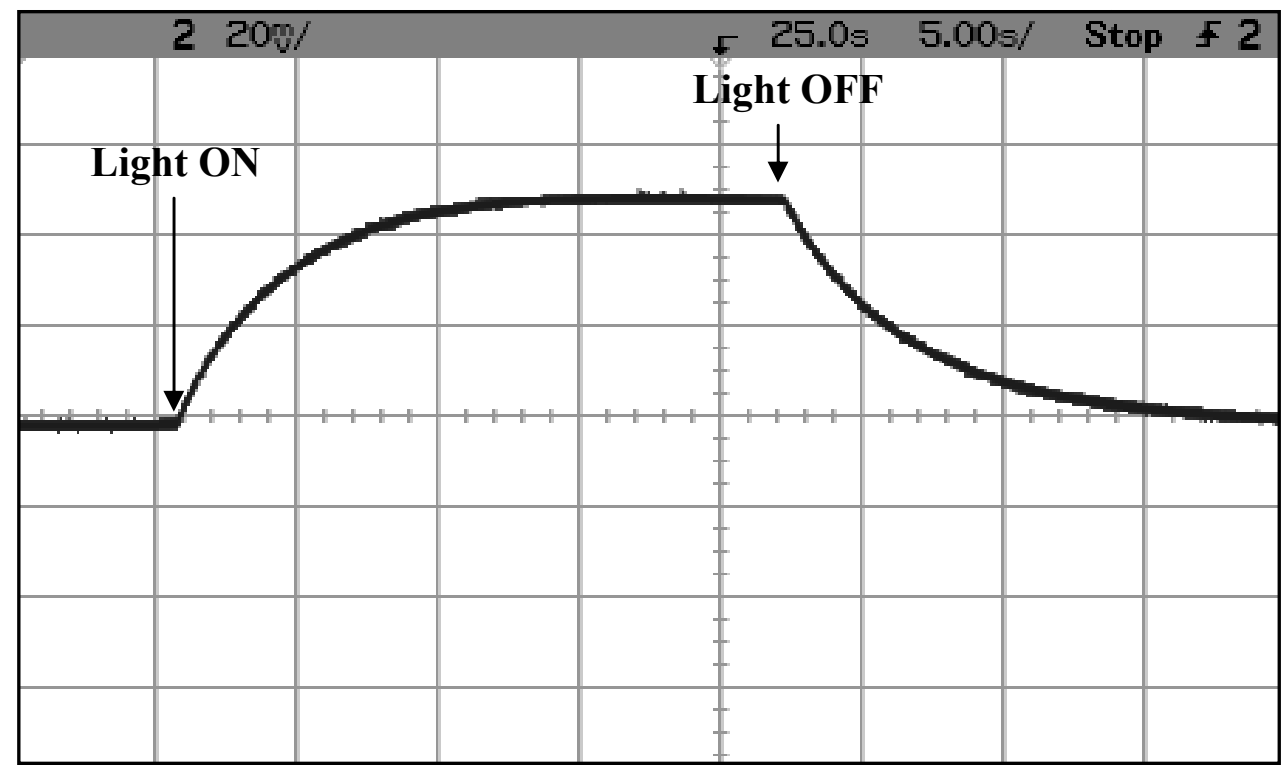

Figure 33. bR Photoelectric response to incadescent light illumination with DC-coupling $(20 \mathrm{mV} / \mathrm{vertical}$ division, 5sec/horizontal division) 
The voltage of the signal can be altered depending upon the power of the illumination source and the amplification factor in the op-amp. For example, by only altering the intensity of the light source, amplified voltage spikes over $0.5 \mathrm{~V}$ have been obtained. An example of high intensity light activation of a bR electrode is shown in Figure 34.

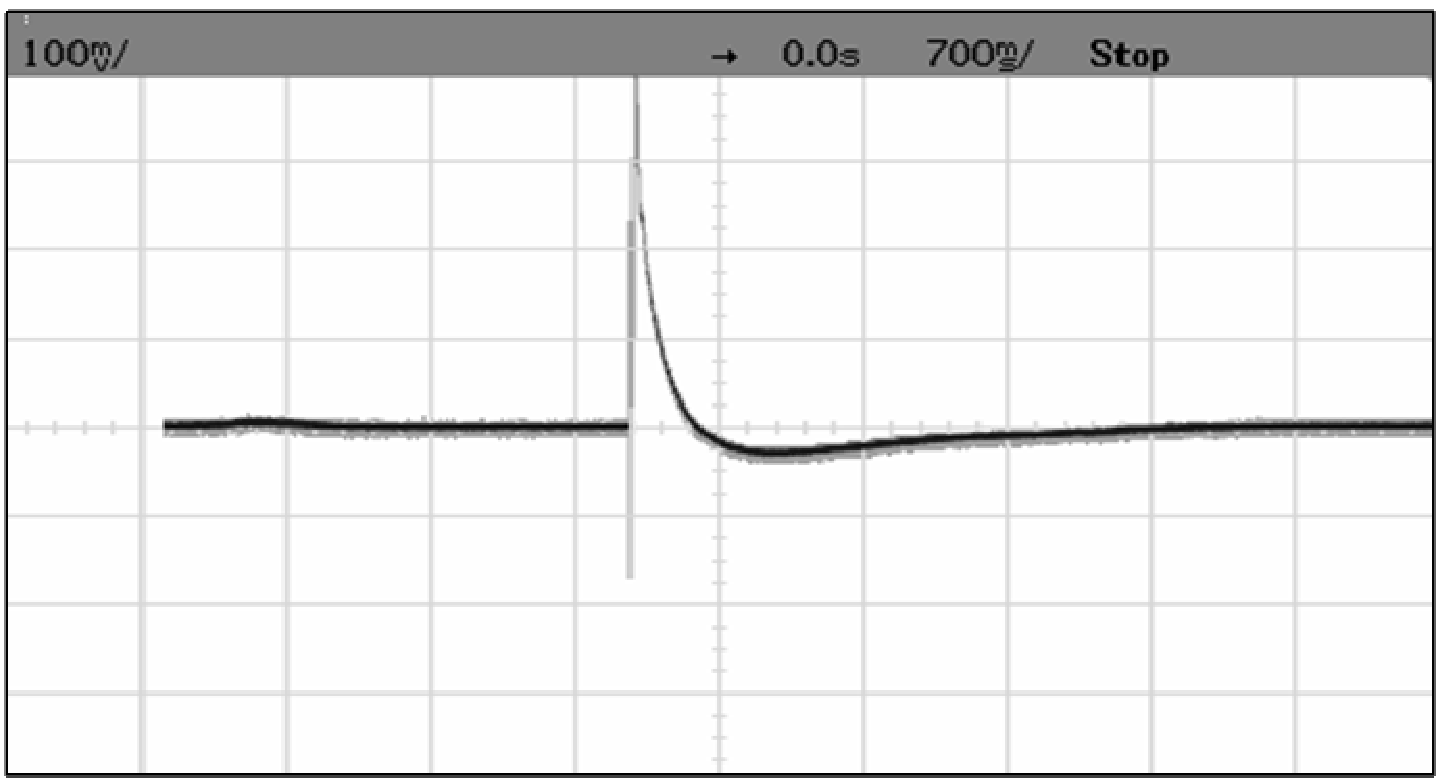

Figure 34. bR photoelectric response to $\sim 8 \mathrm{~mW} / \mathrm{cm} 2$ illumination with $10 \mathrm{x}$ amplification (100mV/vertical division, $0.7 \mathrm{sec} / \mathrm{horizontal}$ division)

The signal has a very sharp spike in voltage output upon illumination with a high intensity camera flash. The sharp nature of this signal is in contrast to the sloping signal seen from the incandescent light, which could be due to the heating of the incandescent filament. As the filament heats up, a more intense light will activate the bR electrode gradually increasing the photovoltage output. With the high intensity flash, the maximum light intensity output was rapidly reached, thus producing a sharp spike in the bR photovoltage output. The length of the flash is on the order of several microseconds, thus it is possible that the full charge movement in each bR molecule hadn't been achieved by the time the light activation had been turned off. Although the bR photocycle is activated in a matter of picoseconds following light illumination, the full 
photocycle (charge movement) takes approximately 10 milliseconds. Therefore, the maximal electrical output might not have been achieved by the time the light activation had ceased. Therefore the distinct negative spike, due to charge migration back to its original location upon termination of light illumination, would start at the peak of the initial signal and decrease back to approximately baseline since the charge motion is of similar distance.

Another interesting aspect of bR electrodes are their rapid cyclic response times. Studies have shown that $\mathrm{bR}$ can provide a detectable photovoltage at a frequency of approximately $100 \mathrm{~Hz}$, correlating to the total photocycle time of $10 \mathrm{~ms}$. Figure 35 displays the bR photovoltaic response to a $4 \mathrm{~Hz}$ signal from an incandescent source.

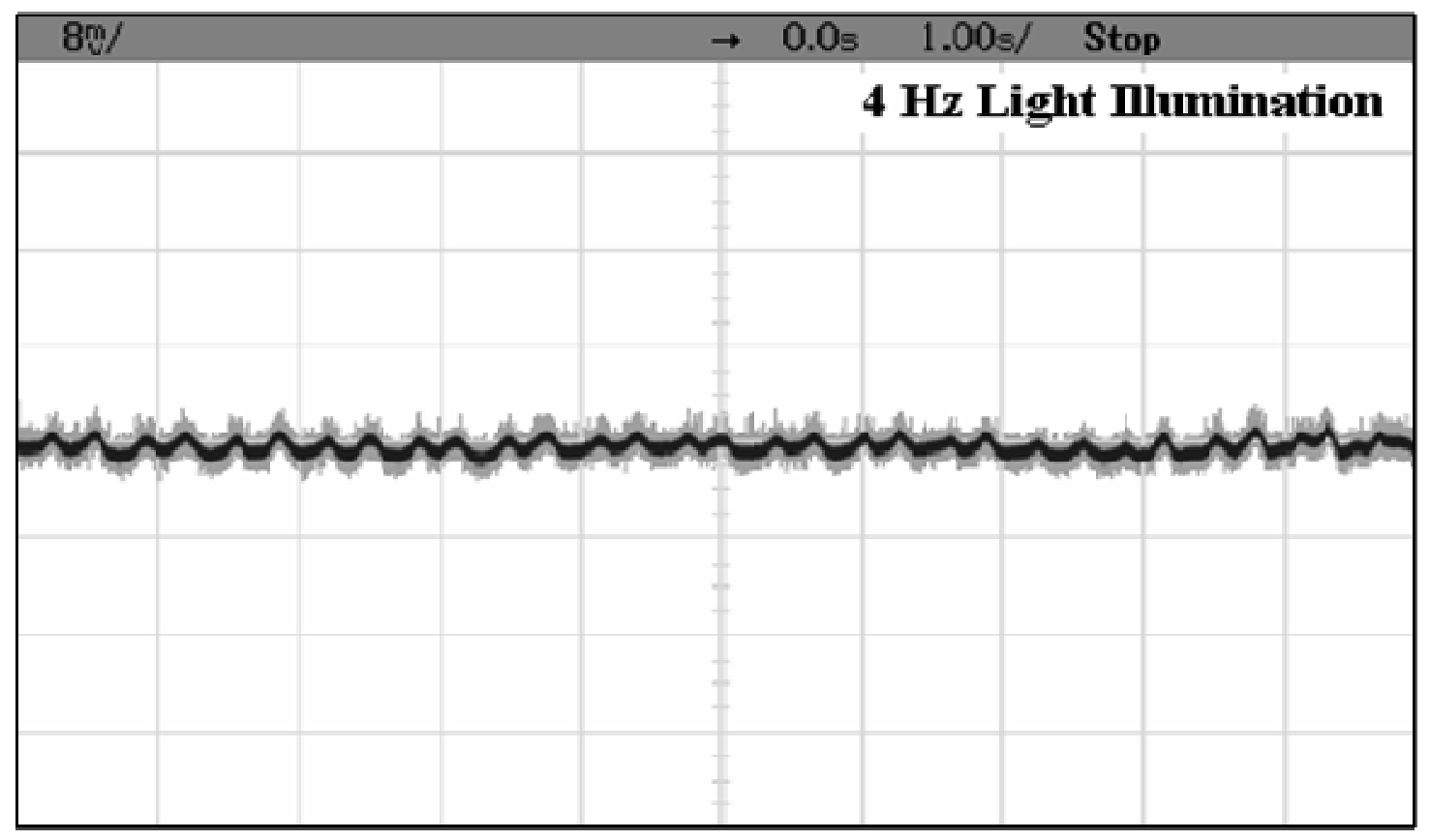

Figure 35. bR photoelectric response to $4 \mathrm{~Hz}$ light illumination (8mV/vertical division, 1sec/horizontal division) 


\subsection{TeMPERATURE DEPENDENCE OF BR PHOTOVOLTAIC RESPONSE}

To implement $b R$ as a phototransducing substrate in a bio-nanosensing device, it will be important to understand the effects of temperature on the bR photovoltaic response. Previous work focusing on bR functionality over a wide range of temperatures has shown that the photocycle intermediates perform faster at higher temperatueres, up to $40^{\circ} \mathrm{C}$, and slows down at near-freezing temperatures, down to $1^{\circ} \mathrm{C}$ [101]. This work presented normalized photovoltage values, thus no clarification on alterations in photovoltage magnitudes over the studied temperature range was given. In a separate study, results conclude that the mechanical stability of the bR molecule decreases dramatically as temperature increases, up to a maximal studied temperature of $52^{\circ} \mathrm{C}$ [102]. It was also found that the largest decrease in mechanical stability occurred as the temperature was increased from $8^{\circ} \mathrm{C}$ to $32^{\circ} \mathrm{C}[102]$.

The impact of temperature on the $\mathrm{bR}$ photovoltaic response was tested on electrodeposited PM-ITO electrodes. In work performed by Karl Walczak and Chris Anton [97], the PM electrode was introduced to elevated temperatures via a hot plate. Once the desired temperature was achieved the sample was removed from the hot plate, the counter ITO electrode was added, and the sample was connected to the operational amplifier for measurement. The PM photovoltage was determined over a temperature range of $22^{\circ} \mathrm{C}$ to $130^{\circ} \mathrm{C}$. The results $\mathrm{PM}$ photovoltaic outputs are plotted in Figure 36 .

It can be seen from Figure 36 that PM photovoltaic response does decrease at elevated temperatures, with the major voltage drop occurring above $80^{\circ} \mathrm{C}$. A complete termination of the bR signal occurs around $120^{\circ} \mathrm{C}$. It can also be noted that smaller PM electrode areas appear to result in a slightly improved resistance to photovoltaic output decay at elevated temperatures. 


\section{Patterned PM Photovoltage vs. Temperature}

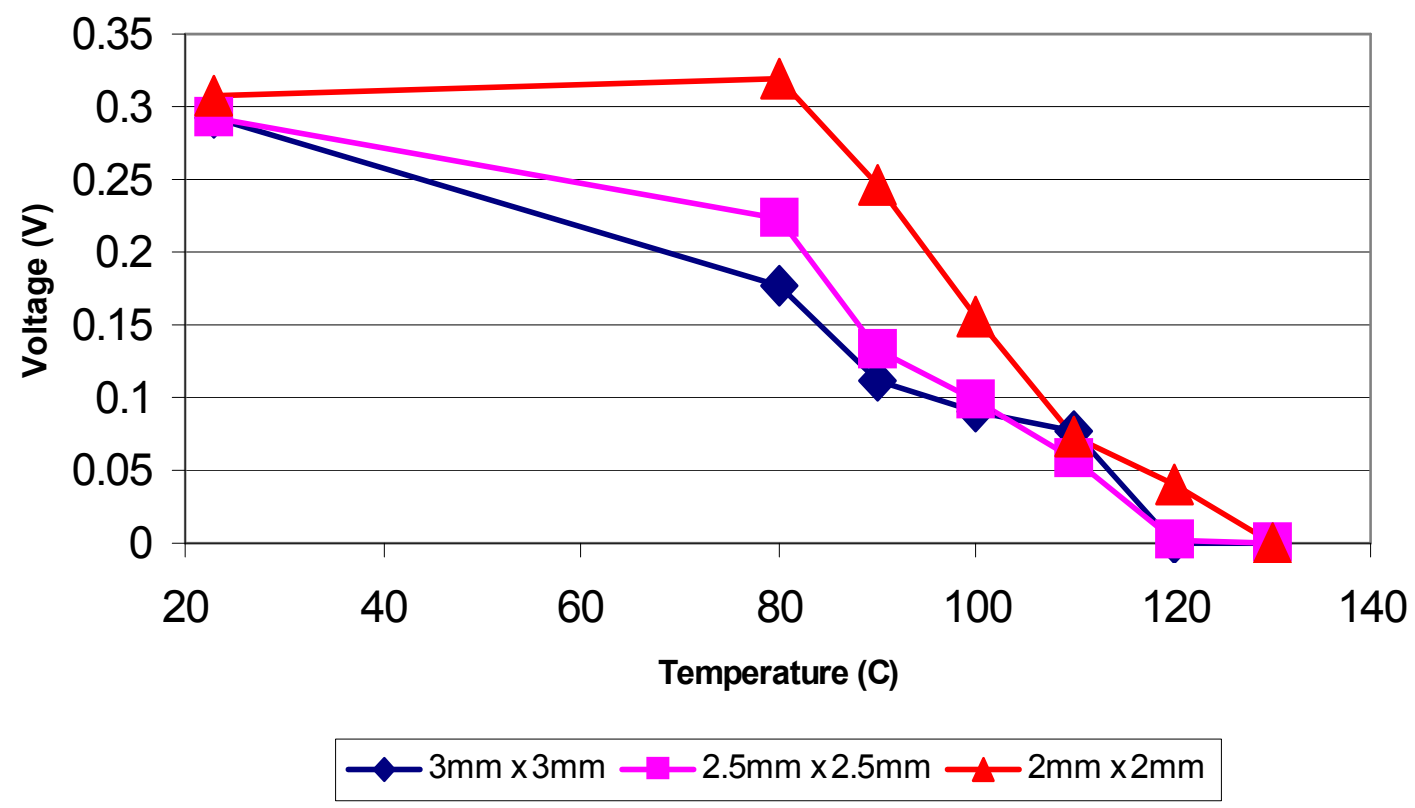

Figure 36. PM photovoltaic response at elevated temperatures [97].

To eliminate any variables associated with separately heating the sample and then performing the photovoltaic measurement at room temperature; a test system was employed to simultaneously heat and measure the PM electrodes. The electrical properties of the assembled electrodes were examined utilizing I-V measurements with the semiconductor (SC) analyzer system (Janis/Keithley-4200; Micro Manipulated Cryogenic Probe System). Instead of utilizing a separate ITO electrode as the counterelectrode, a $5 \mathrm{~nm}$ thin layer of gold was sputtered directly on top of the PM sample. Care was taken to ensure the gold was only sputtered on the PM surface and not any of the ITO to avoid short-circuiting the system. A two-probe system was used to contact the bottom ITO electrode and top gold electrode separately. The PM photoresponse was measured through an amplifier built into the Keithley-4200 unit. This unit, located in Dr. Shashi Karna's lab at the Aberdeen Proving Grounds, allowed for precise temperature control in the $3 \mathrm{~K}$ to $475 \mathrm{~K}$ range. 
Once connected and sealed into the temperature controlled probe station the bR was light-adapted for 10 minutes and an initial photovoltage measurement was taken. In Figure 37 the temperature was increased and the bR photovoltage was measured in 2.5 Kelvin intervals. A plot of the actual bR photovoltaic waveform over the temperature scan is displayed in Figure 38. The response time for temperature increase is very rapid in the probe system, thus the sample was allowed to equilibrate to the new temperature for 5 minutes before the photovoltage reading was taken. The temperature was raised to a maximum of $310 \mathrm{Kelvin}$, or $36.85^{\circ} \mathrm{C}$, immediately followed by an incremental decrease in temperature of 2.5 Kelvins per measurement. No liquid nitrogen was connected to the system during the elevated temperature study, thus the sample chamber cooled more slowly and each incremental measurement was approximately 15 minutes apart.

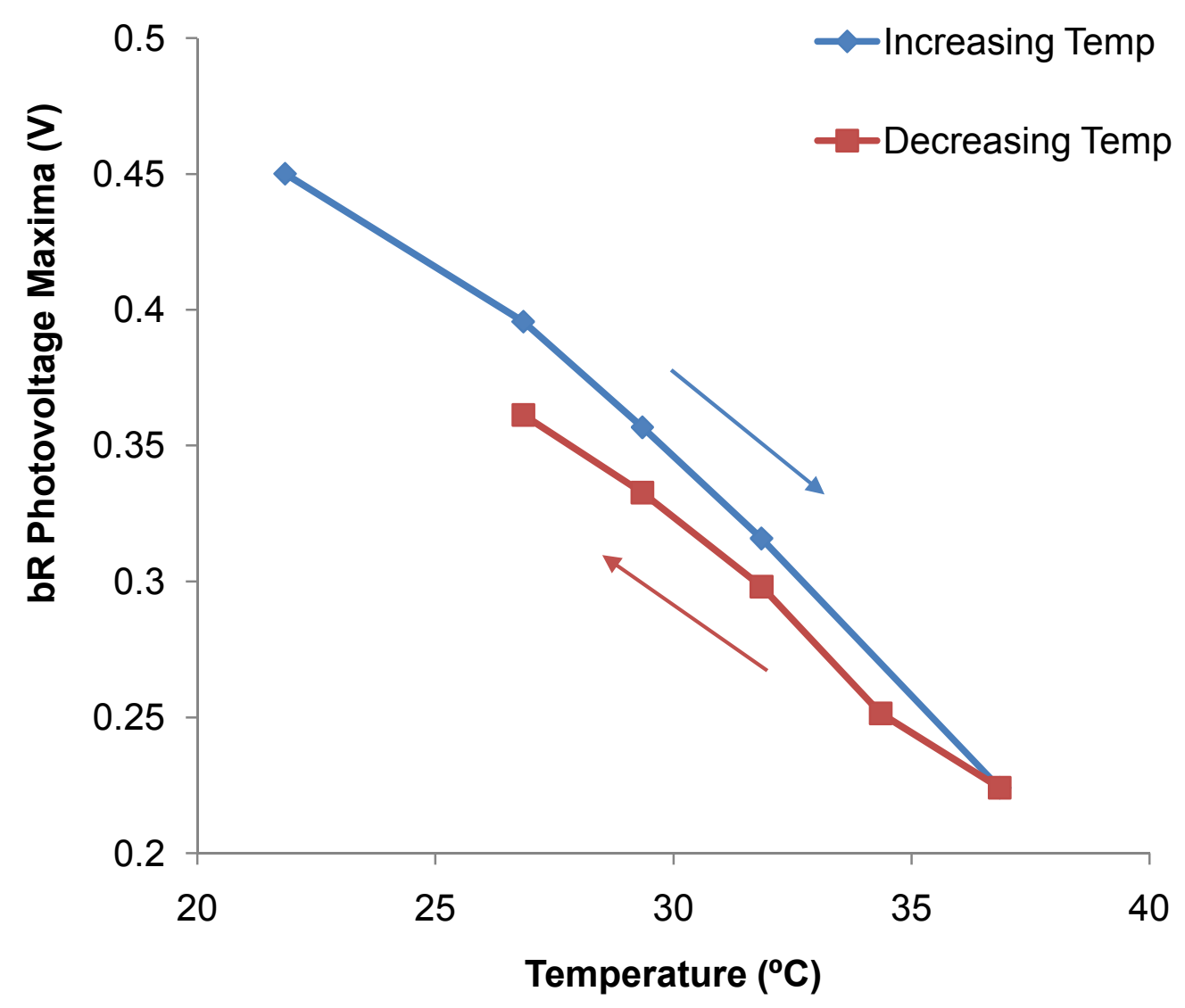

Figure 37. Effect of temperature increase on bR photovoltaic response and subsequent recovery. 


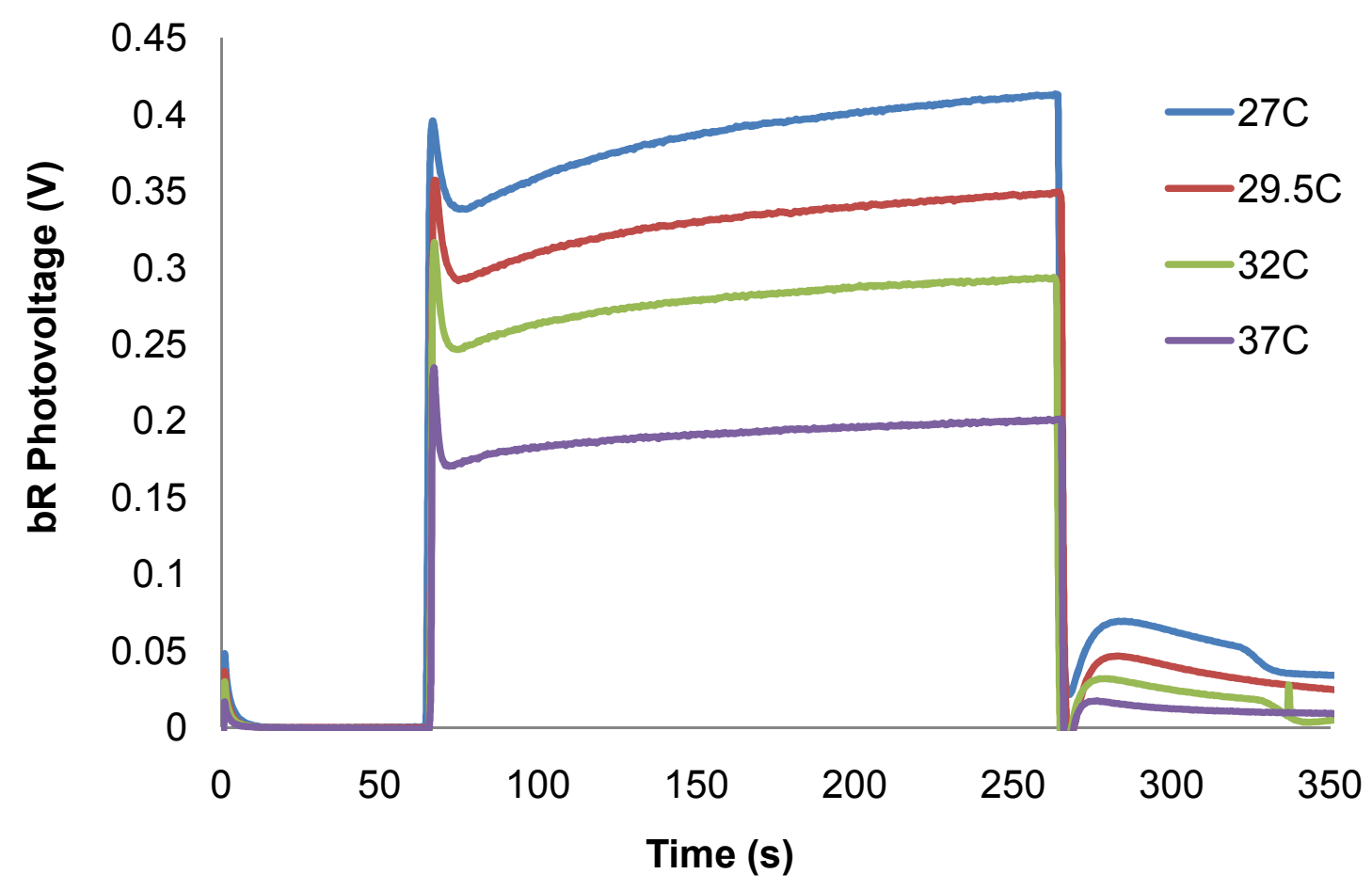

Figure 38. Waveform of $b R$ photovoltaic response during temperature increase.

It can be seen from Figure 37 that the increase in temperature appears to have a larger affect on the bR photovoltaic output than previously determined in Figure 36. This is possibly due to the constant temperature held on the sample in the current study which did not allow for any cooling period before the photovoltage measurement was taken. Plotting the bR photovoltage vs. temperature also suggests that the photovoltage begins to decrease more rapidly with each incremental temperature increase. This is evidenced by an initial photovoltage decay rate of $-10.8 \mathrm{mV} /{ }^{\circ} \mathrm{C}$ in the $21.85^{\circ} \mathrm{C}$ to $26.85^{\circ} \mathrm{C}$ range, concluded by a $-18.3 \mathrm{mV} /{ }^{\circ} \mathrm{C}$ photovoltage decay rate in the $31.85^{\circ} \mathrm{C}$ to $36.85^{\circ} \mathrm{C}$ range.

In addition to a temperature increase, this study also evaluated the recovery of the bR photovoltaic response as the temperature was decreased to room temperature. In Figure 37 it can be seen that the recovery of the bR photovoltage tracks closely to its initial output at the given temperature. Each temperature point measured in the recovery cycle regained over $90 \%$ of its original photovoltage magnitude. This shows that the increase in temperature had minimal, if any, long-term effects on the bR photovoltaic response 
and could be fully recovered in the temperature range scanned. More studies will need to be performed in this area to determine the full range of bR activity at elevated temperatures.

To analyze the $\mathrm{bR}$ photovoltaic response at lowered temperatures the same temperaturecontrolled probe setup was used. A new bR sample was used from the previous study, although prepared with a gold top electrode in the same manner. Once connected and sealed into the temperature controlled probe station the $\mathrm{bR}$ was light-adapted for 10 minutes and an initial photovoltage measurement was taken. The liquid nitrogen line was connected to facilitate efficient cooling of the sample. The response time for temperature decrease was very rapid in the probe system, thus the sample was allowed to equilibrate to the new temperature for 5 minutes before the photovoltage reading was taken. The temperature was lowered in approximately 3 Kelvin increments to a minimum of 255 Kelvin, or $-17.78^{\circ} \mathrm{C}$, immediately followed by an incremental increase in temperature and subsequent measurement. The effect of decreased environmental temperatures on the $\mathrm{bR}$ photovoltaic response is plotted in Figure 39.

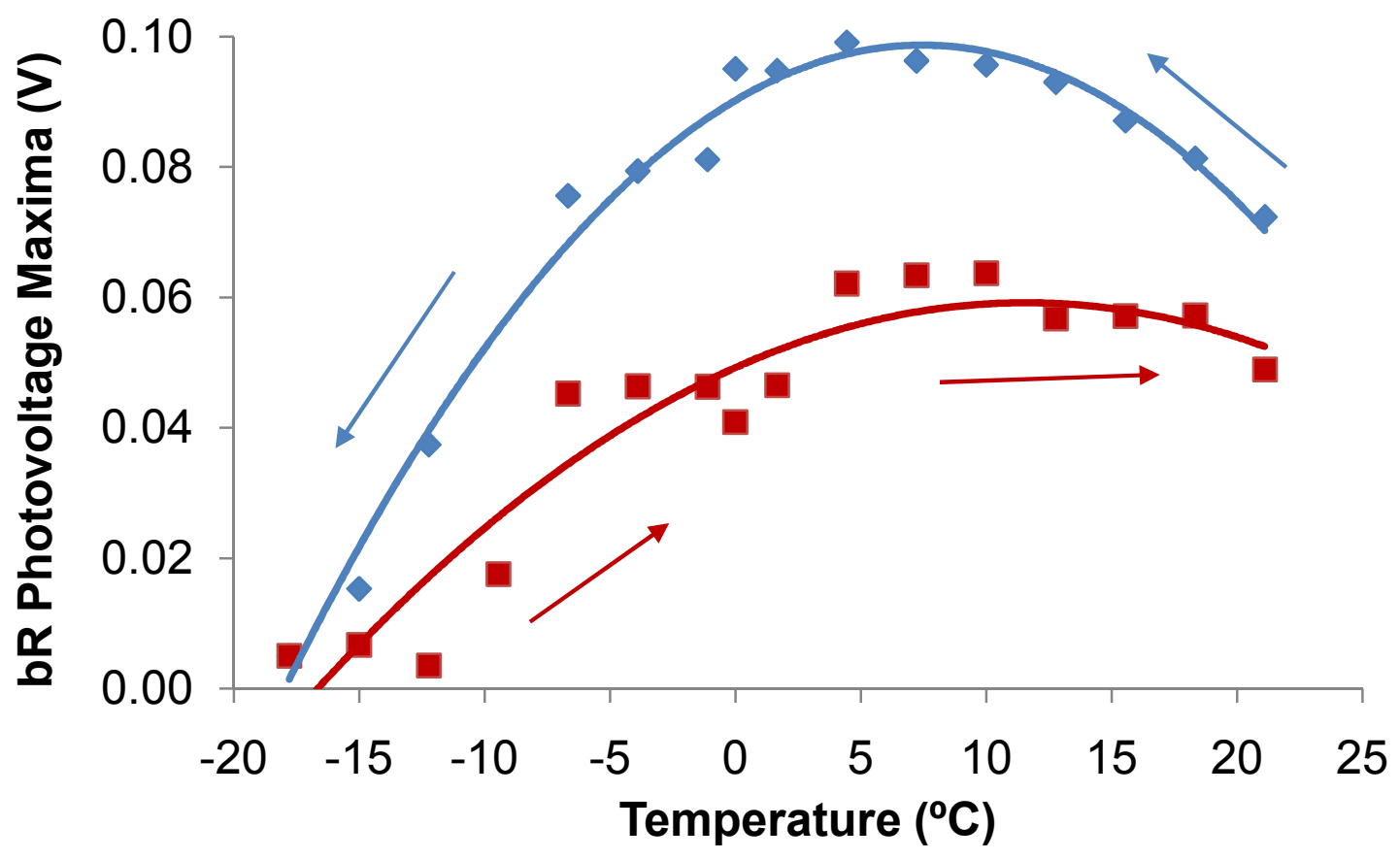

Figure 39. Effect of decreasing temperature on bR photovoltaic response and subsequent recovery. 
The data suggests that room temperature is not the optimal operating temperature to achieve maximal bR output. The fitted trendline suggest the photovoltage maximum occurs at approximately $5^{\circ} \mathrm{C}$ to $10^{\circ} \mathrm{C}$, resulting in a $37 \%$ increase in photovoltage output over room temperature measurements. Incremental reductions in temperature below $5^{\circ} \mathrm{C}$ resulted in a rapid decrease in $\mathrm{bR}$ photovoltage. An elimination of bR photovoltage was achieved around $-20^{\circ} \mathrm{C}$. It is possible that frozen water particles within the bR molecule inhibit proton translocation, although large signals are still seen at sub-freezing temperatures. An important note at sub-freezing is that the waveform changes from transient to steady-state, as shown in Figure 40. This data suggests that the $b R$ impedance decreases with lowered temperatures.

Upon increasing the temperature back to room temperature, the bR electrode regained a portion of its initial photovoltaic response, as shown by the red curve in Figure 39. Temperature was increased rapidly to each test point and the bR sample was allowed to equilibrate to the new temperature for 5 minutes before taking the photovoltage measurement. This is a much shorter recovery transition time, as compared to the 15 minutes given in the elevated temperature recovery, and resulted in a relatively smaller percentage recovery. Specifically the recovery from sub-freezing temperatures resulted in a $61 \%$ average photovoltage recovery, compared to the $90 \%$ recovery to elevated temperatures. Since the elevated temperature sample was allowed approximately three times more recovery time, it is unclear whether the decreased photovoltaic recovery in frozen samples is due to less recovery time or a greater degree of damaged imparted on the bR molecule. 


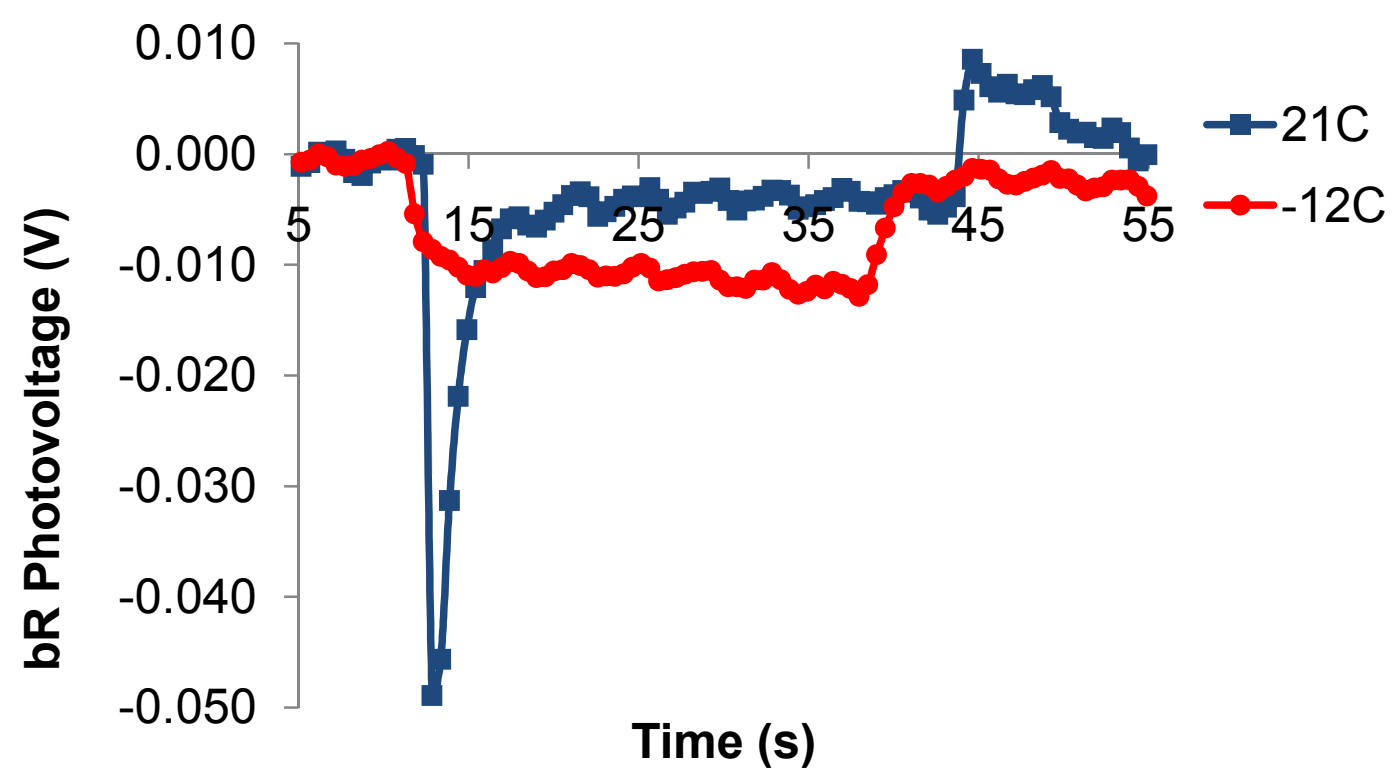

Figure 40. Alterations in bR photovoltaic waveform based on temperature. The same bR sample displays a differential photovoltaic response at $21^{\circ} \mathrm{C}\left(70^{\circ} \mathrm{F}\right)$, with the response waveform changing to steady-state voltage at sub-freezing temperatures.

It has been shown that the bR photovoltaic waveform is directly related to the comparative values of bR film impedance and the input impedance of the voltage measurement circuitry [24]. Specifically it was found that utilizing a measurement circuit with input impedance 1-order of magnitude lower than the bR film resistance results in a differential photovoltaic response. This is the same photovoltaic waveform displayed by the bR at $21^{\circ} \mathrm{C}$ in Figure 40 .

With a measurement circuit input impedance 1-order of magnitude larger than the $b R$ film resistance results in a steady state output, similar to that seen by the same bR sample at $-12^{\circ} \mathrm{C}$ in Figure 40 . Since the input impedance of the measurement circuit was not changed during the measurements, the alteration in waveforms is potentially due to changes in the bR film impedance. These results suggest that the bR film impedance decreases with temperature, potentially by up to 2 -orders of magnitude. 


\section{Chapter 4. QD Activation of bR Photovoltaic Response}

To utilize photovoltaic properties of $b R$ in a future array, there's a need to have an activation light source that is specific to individual bR sites. The use of quantum dots as this light source is being investigated due to their nanometer size and tunable wavelength emission. The ideal photonic absorption wavelength for quantum dots occurs in the UV region. The quantum dots chosen for this study were purchased from Evident Technologies with photonic emissions near 570nm. As shown in Figure 41, these particular quantum dots were selected due to their emission wavelength correlation with the maximal bR absorption point.

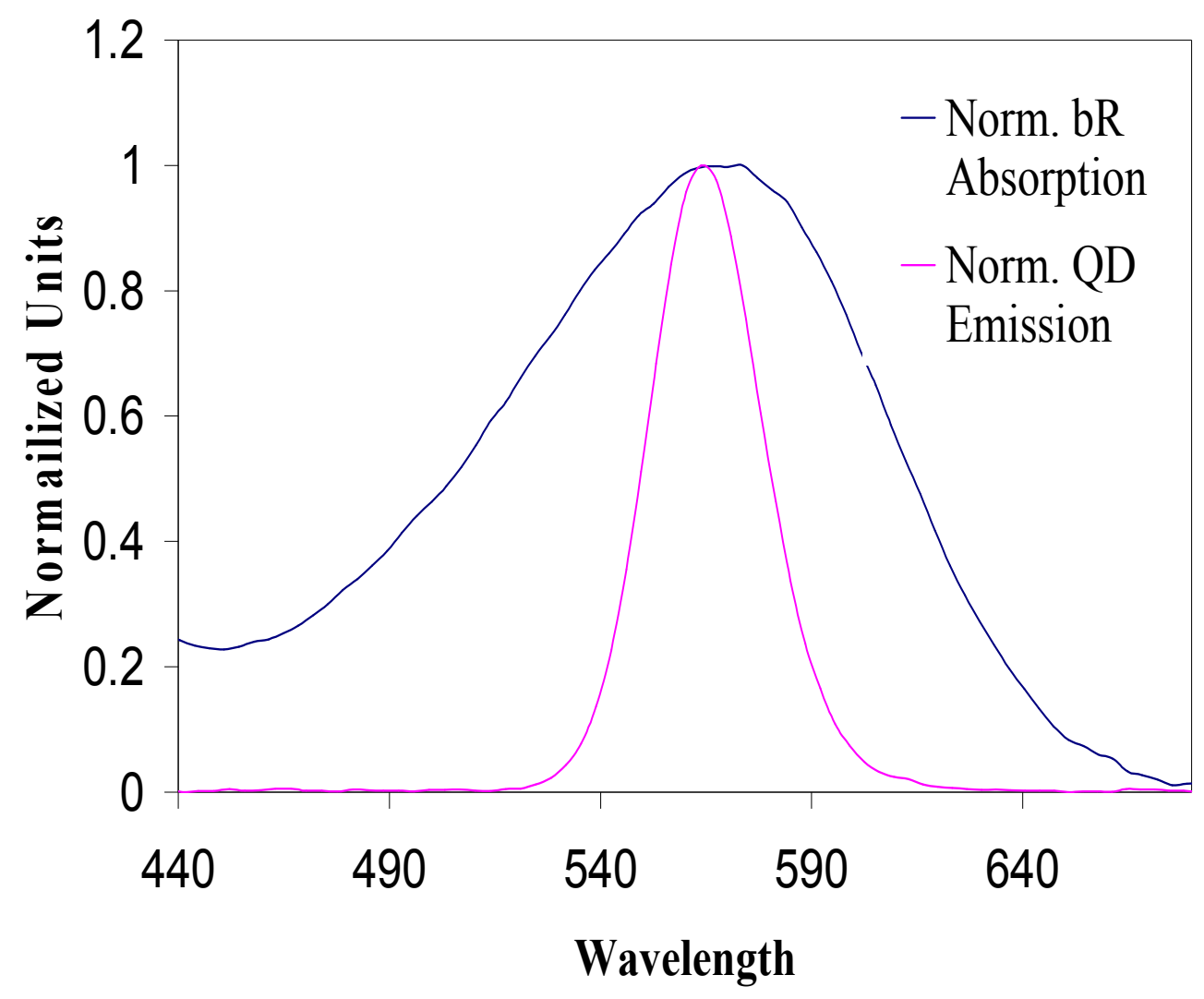

Figure 41. 565nm QD emission spectra and bR absorption spectra overlap 


\subsection{EFFECTS OF CONTINUOUS UV ILLUMINATION ON BR PHOTOVOLTAGE}

Since the quantum dots will be activated with UV light, the effect of UV light illumination on the electrical output of bR was evaluated. Using continuous UV (340nm) light illumination on a bR electrode, the electrode's photovoltaic response to visible light was measured over a span of 95 minutes of UV illumination. The visible light source used in these studies was a camera flash, which produced a light intensity of approximately $8 \mathrm{~mW} / \mathrm{cm}^{2}$. The initial and final photovoltage measurements are shown in Figure 42 and Figure 43 respectively.

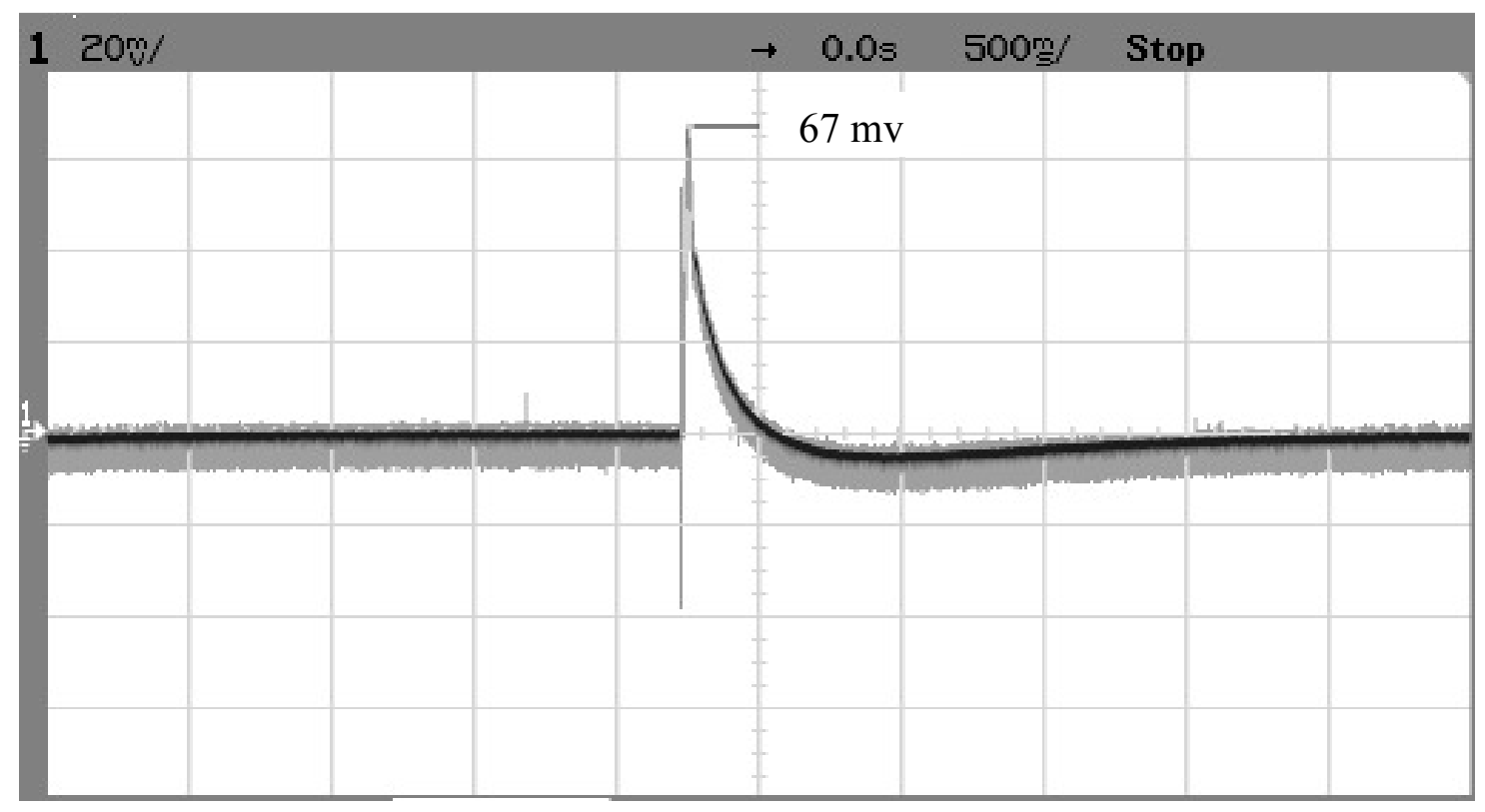

Figure 42. bR electrode signal from $8 \mathrm{~mW} / \mathrm{cm} 2$ light illumination before $U V$ illumination $(t=0)$ (20mV/vertical division, $0.5 \mathrm{sec} /$ horizontal division) 


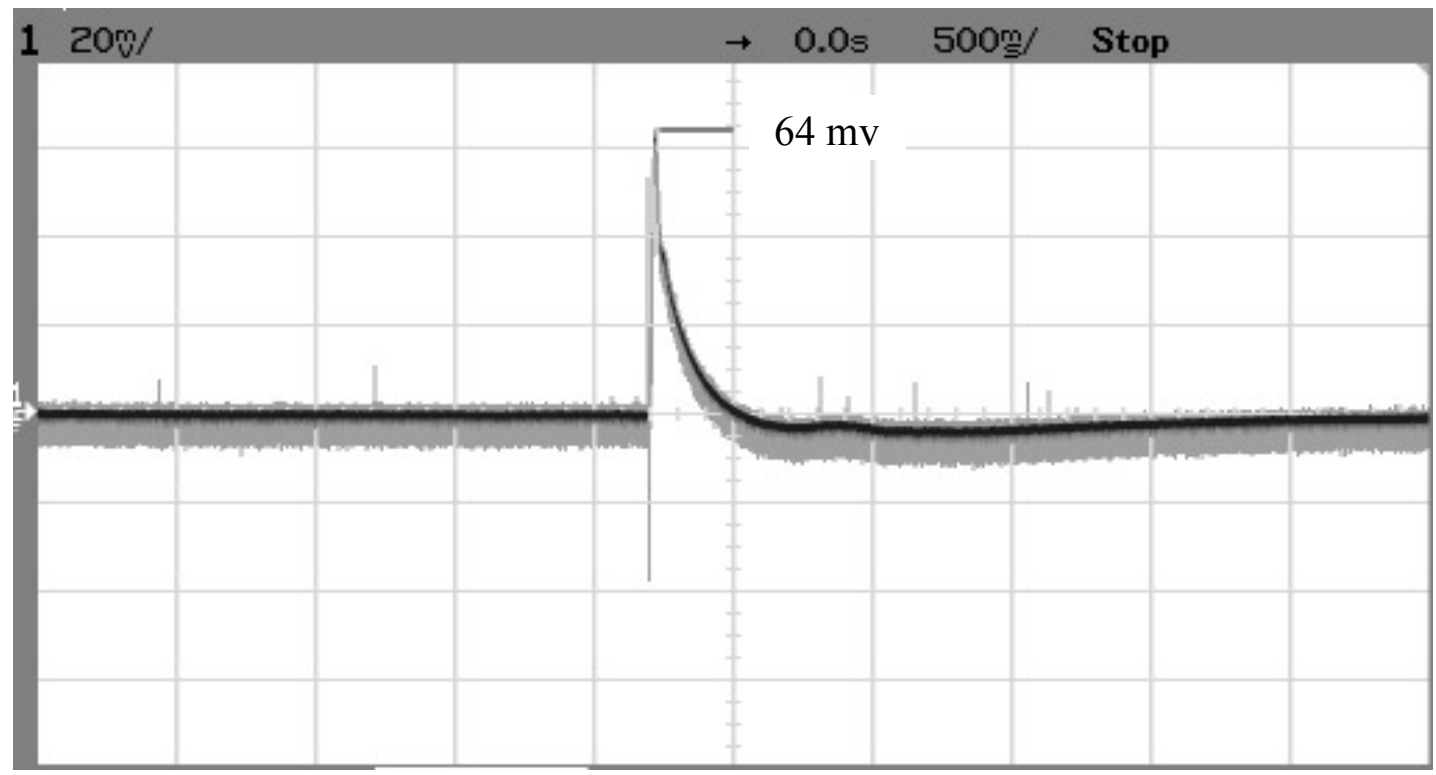

Figure 43. bR electrode signal from $8 \mathrm{~mW} / \mathrm{cm} 2$ light illumination following $\mathrm{UV}$ illumination $(\mathrm{t}=\mathbf{9 5}$ minutes) (20mV/vertical division, $0.5 \mathrm{sec} /$ horizontal division)

The drop in photovoltage of $3 \mathrm{mV}$ seen from the first to the last measurements is minor since this variation is within the range of signals measured throughout the study. Therefore this study demonstrated that constant UV illumination should have no significant effects on the magnitude of the bR electrical response to visible light. In an actual sensing system, it is anticipated that QD excitation would be from a pulsed UVLED source to conserve power.

\subsection{BR PHOTOELECTRIC RESPONSE FROM QD ILLUMINATION}

To test the capability of quantum dot photonic emission to activate the bR photocycle, the apparatus shown in Figure 44 was constructed. This allowed for the photoluminescence from the QD emission to easily interact with the bR electrode. For this study, a 310nm UV-LED light source was used to excite the QD electron-hole pair and ultimately lead to photon emission. The spacing between the bR and QDs precludes FRET coupling and 
isolates QD photonic activation of bR. The sharp UV emission from the LED is shown in Figure 45.

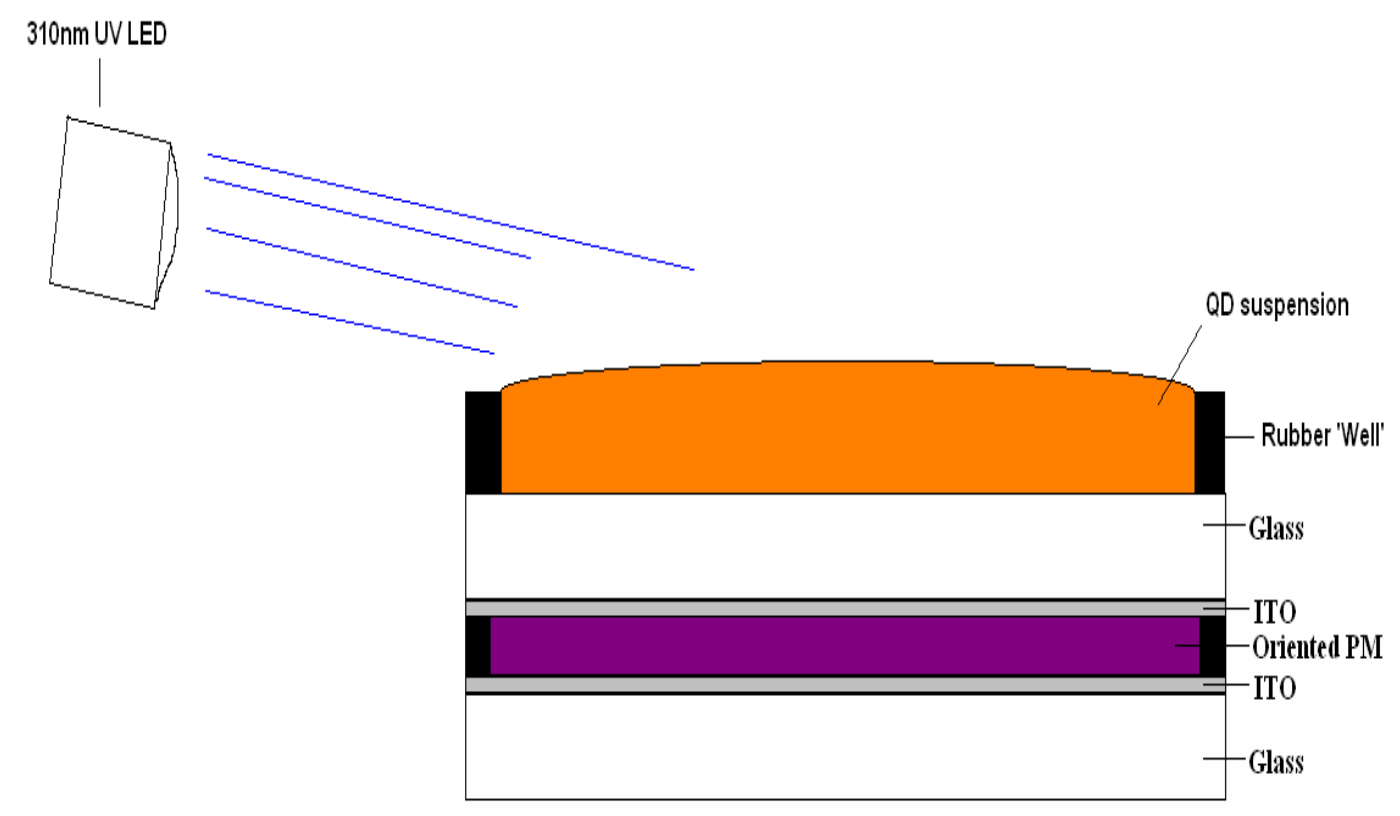

Figure 44. Schematic of QD activation test fixture

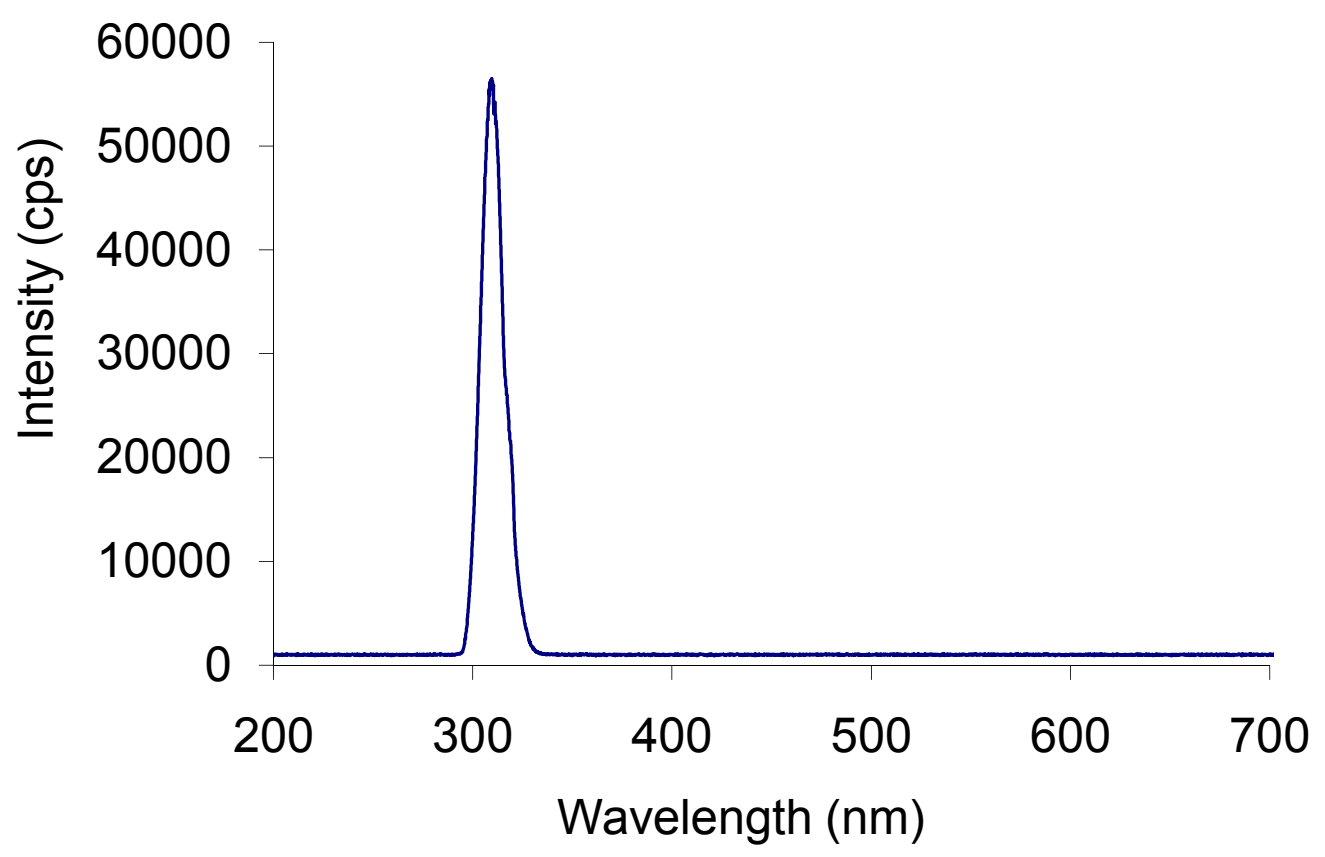

Figure 45. 310nm UV LED emission spectrum 
Previous work has shown that the energy of UV light, absorbed by the aromatic amino acids in $\mathrm{bR}$, can be transferred to the retinal through resonance and initiate the photocycle [103]. Therefore the angle of the UV source was adjusted to avoid bR illumination and activation of the bR photocycle. In the control experiment, the LED was repeatedly flashed at a frequency of approximately $4 \mathrm{~Hz}$ without the addition of the QD suspension to the bR electrode. The electrical response of the system during the UV flashing is shown in Figure 46. No noticeable cyclic electrical signal was observed.

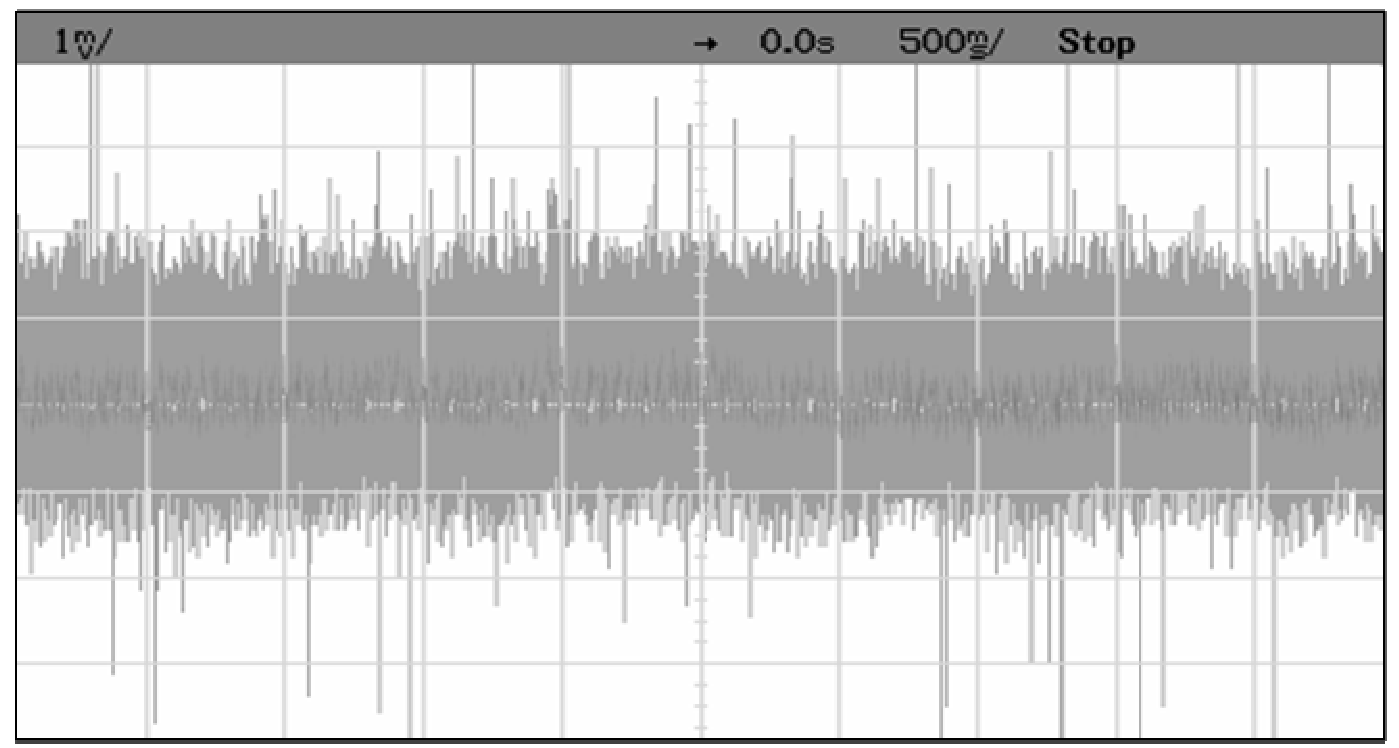

Figure 46. bR photoelectric response to $4 \mathrm{~Hz}$ UV flashing - no QD's $(1 \mathrm{mV} /$ vertical division, $500 \mathrm{~ms} /$ horizontal division)

A $40 \mu \mathrm{L}$ volume of $63 \mathrm{nmol} / \mathrm{L}$ QD suspension was applied in the well above the bR electrode. Upon UV flashing at approximately $4 \mathrm{~Hz}$, the QDs were visibly activated and their photonic emission resulted in the bR signal shown in Figure 47. The cyclic signal generated from the $b R$ electrode directly correlated to the flashing of the UV-LED. 


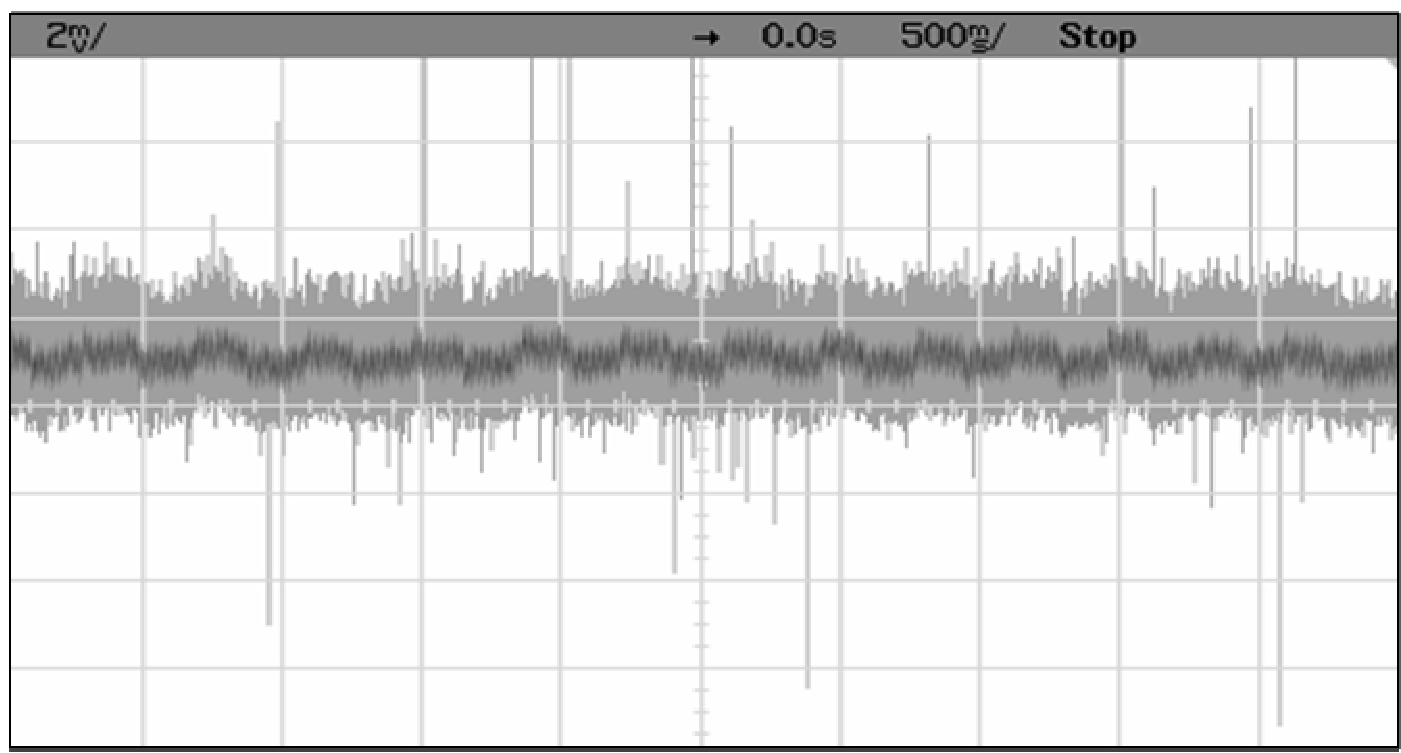

Figure 47. bR photoelectric response to $4 \mathrm{~Hz}$ QD illumination ( $2 \mathrm{mv} /$ vertical division, $500 \mathrm{~ms} /$ horizontal division)

This was the first known disclosure of the activation of bR with a quantum dot illumination source. In the test system, it was calculated that there were $1.52 \times 10^{15}$ quantum dots $\left(40 \mu \mathrm{L}\right.$ of $63 \mathrm{nmol} / \mathrm{mL}$ QD suspension) compared to the $1.38 \times 10^{16} \mathrm{bR}$ molecules $(40 \mu \mathrm{L}$ of $15 \mathrm{mg} / \mathrm{mL}$ PM solution used for EPS) in the oriented film. This corresponds to approximately $1 \mathrm{QD}$ for every $10 \mathrm{bR}$ molecules in the system. Assuming that every bR molecule was photonically activated, then each quantum dot was activating several bR molecules. This could show the potential capability of utilizing quantum dots in a nano-scale system. This is due to the fact that a single monolayer of quantum dots might activate several monolayers of PM, resulting in a larger electrical output.

\subsection{THIN FILM QD ACTIVATION OF BR PHOTORESPONSE}

In the previous study the QDs were suspended in a well above the PM electrode to achieve bR activation from the QD photon emission. For sensor applications, however, the QDs must be applied near the PM electrode in dried film form so it is capable of 
interacting and binding with airborne target. . This study looked at the potential of utilizing biotinylated $\mathrm{bR}$ as the attachement mechanism to bind streptavidin-coated quantum dots to the PM-based electrode.

The attachment of QDs to the PM electrode was accomplished through the use of a biotin-streptavidin linkage. The small biotin molecule has an extremely high binding affinity for the streptavidin protein, to help ensure specific and tight binding at all locations. Streptavidin, a 53kDa protein, and biotin, also known as vitamin $\mathrm{H}_{\text {or }} \mathrm{B}_{7}$, are together widely regarded as the strongest non-covalent bond in nature, with a dissociation constant $\left(\mathrm{K}_{\mathrm{d}}\right)$ of $4 \times 10^{-14} \mathrm{M}$ [104]. The dissociation constant measures the concentration of the ligand at which half of the protein binding site is occupied. Thus with a $K_{d}$ of $4 \mathrm{x}$ $10^{-14} \mathrm{M}$, or $0.4 \mathrm{fM}$, the biotin-streptavidin system has a higher binding strength than the average antigen-antibody system, which has a $\mathrm{K}_{\mathrm{d}} 10^{-7} \mathrm{M}$ to $10^{-11} \mathrm{M}$. Breaking this bond can only be achieved with harsh treatments or environmental temperatures in excess of $70^{\circ} \mathrm{C}[105]$. With its robust nature and virtually unbreakable bond, the biotin-streptavidin binding scheme has been well studied in applications of nanoparticle linkers [106-110]. In these studies the biotin-streptavidin linkage was utilized to link biotinylated bR with streptavidin coated quantum dots.

The first step in this procedure was to biotinylate the bR molecule, which has been explored by various research groups [111-113]. The PM fragments to be biotinylated were suspended in freshly made $0.1 \mathrm{M}$ sodium bicarbonate buffer; previously adjusted to pH 8.4 with $5 \mathrm{M} \mathrm{KOH}$. The PM was suspended to a concentration of $2.5 \mathrm{mg} / \mathrm{ml}$. The solution was then lightly sonicated to break up any large PM aggregrates and the $\mathrm{pH}$ was re-checked to ensure a proper $\mathrm{pH}$. A total volume of $115 \mu 1$ of the prepared PM suspension was removed and placed in a microcentrifuge tube on ice.

The biotin was prepared once the PM was ready for biotinylation. In a separate tube, on ice, $100 \mu 1$ of chilled sterile water was added. A total of $0.83 \mathrm{mg}$ of biotin (MW 556.58) was added to the tube and pipetted up and down to ensure the biotin was completely dissolved. This biotin concentration led to a 27:1 biotin:bR molar ratio in the final 
mixture. The tube containing the biotin solution was then vortexed and briefly microcentrifuged to collect droplets on the tube wall.

At this point the biotin was ready to be added to the PM solution. A total of $20 \mu 1$ of the biotin solution was pipetted into the prepared PM solution and mixed by pipetting up and down. The mixed solution was briefly microcentrifuged to remove droplets and then the reaction was allowed to run for 1 hour at room temperature. Following biotinylation the excess biotin was removed through a desalting column. The biotinylated bR could be used immediately or stored at $-80^{\circ} \mathrm{C}$ for future studies.

This work looked at two methods to achieve a biotinylated bR surface to bind to the streptavidin-coated QDs. These two methods focus on 'pre' and 'post' bR biotinylation procedures. The pre-biotinylated procedure involves biotinylating the bR using previously reported techniques. This resulted in a liquid suspension of PM fragments containing covalently bound biotin groups on the $b R$ molecules. The biotinylated $b R$ is then put into electrode form using the electrophoretic sedimentation technique.

The post-biotinylated bR-based electrode is created by first making a bR electrode using the electrophoretic sedimentation technique. Specifically $30 \mu \mathrm{L}$ of $5 \mathrm{mg} / \mathrm{ml} \mathrm{PM}$ was deposited at $1 \mathrm{~mm}$ separation at $40 \mathrm{~V} / \mathrm{cm}$ for 1 minute. Once dried, $10 \mu 1 \mathrm{of} 5 \mathrm{mg} / \mathrm{ml}$ biotin solution $(\mathrm{pH} 8$ ) is deposited on the top surface of the bR electrode and incubated at room temperature for 1 hour. The sample is rinsed in deionized distilled (ddI) water for 5 minutes to remove any unbound biotin molecules.

To determine the presence and functionality of the biotin groups for both 'pre' and 'post' biotinylated bR-based electrodes, a $10 \mu 1$ solution of $0.5 \mathrm{nmol} / \mathrm{ml}$ streptavidin-coated QDs (595nm emission) was incubated on the biotinylated bR electrode surface for 15 minutes. The samples were then rinsed for 2 minutes in ddI water to remove any unbound streptavidin-coated QDs. The fluorescence emission of the samples was then tested on the Hitachi-F7000 fluorospectrometer to determine the presence of bound QDs. The resulting fluorescence spectrum for each sample type is shown in Figure 48. 


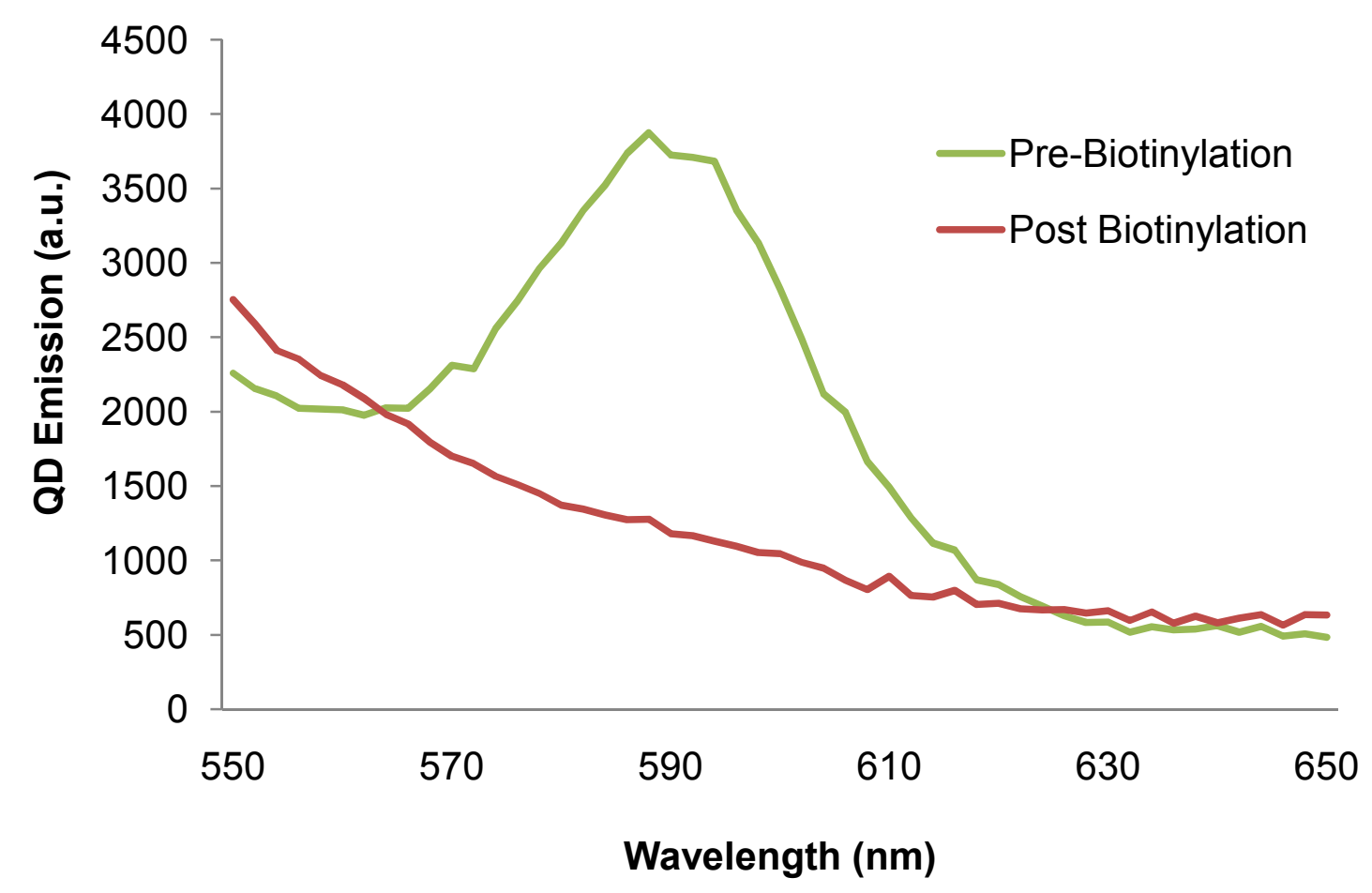

Figure 48. Emission spectra of pre and post biotinylated bR electrodes after addition of $595 \mathrm{~nm}$ streptavidin-coated QDs

These initial results show that pre-biotinylated bR electrodes retain the capacity to effectively bind to a functional streptavidin group. The post-biotinylated bR electrode, however, showed no binding capability to streptavidin. This lack of functionality is likely due to the post-biotinylation procedure and could be further studied to facilitate biotinylation of the dried films.

In addition, the electrical activity of pre-biotinylated bR-based electrodes was also confirmed. These electrodes displayed a strong electrical response to Xenon lamp illumination, resulting in DC signals of approximately $4 \mathrm{~V}$ and $\mathrm{AC}$ signals of over $200 \mathrm{mV}$. The bR photovoltaic response of the pre-biotinylated electrodes with and without QD addition is shown in Figure 49. 


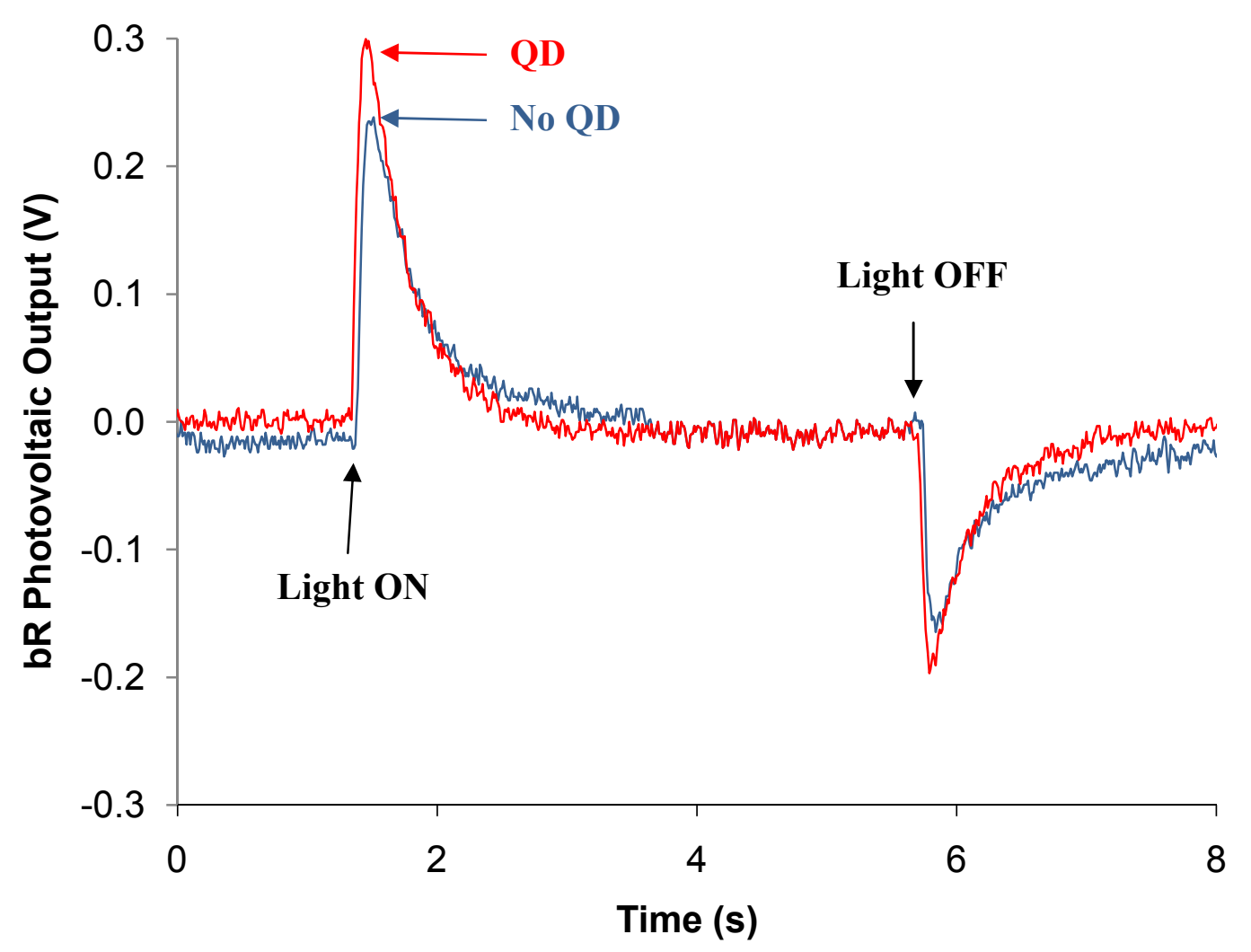

Figure 49. Photovoltaic response of pre-biotinylated bR electrode with and without QD addition.

It can be seen in Figure 49 that the binding of the QDs to the surface of the PM electrode resulted in an increased bR photovoltaic response to white light illumination. Without any QDs present, the bR electrode yielded a $0.24 \mathrm{~V}$ response upon light illumination followed by a $-0.17 \mathrm{~V}$ spike upon termination of the light source. When a QD layer was then bound to the same sample and re-activated, the initial spike yielded a bR photovoltage of $0.31 \mathrm{~V}$ followed by a $-0.2 \mathrm{~V}$ spike upon termination of the light source. These outputs yielded an on/off peak ratio of 1.42 and 1.55 for the bR and bR-QD electrodes. In total the addition of the QDs to the PM electrode increased the $\mathrm{bR}$ photovoltaic output by approximately $70 \mathrm{mV}$, or $34.3 \%$. These studies conclude that QDs can enhance the bR photovoltaic response, even with white light illumination. The photovoltage increase of $34.3 \%$ is potentially large enough that alterations to the QD emission could affect the bR output on a detectable scale, creating the possibility for sensor applications. 


\section{Chapter 5. QD-bR Hybrid Ionic-Self Assembled Monolayers}

To efficiently utilize QDs as an activation source for a bR photoresponse, the two nanomaterials must be integrated within nanoscale proximity. As displayed in Figure 50, the proposed sensing system will utilize the QD photonic emission to excite the bR photoresponse.

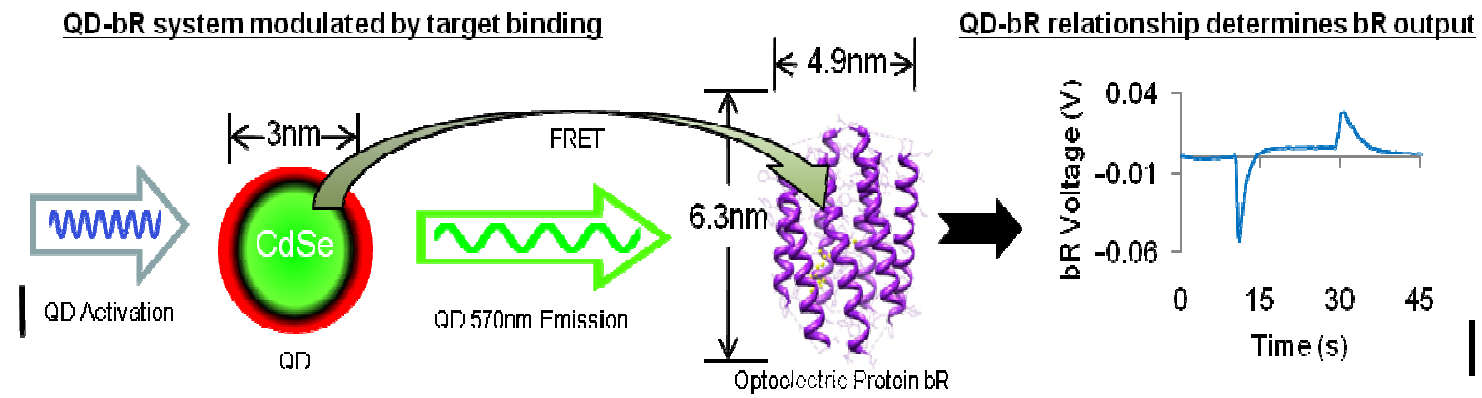

Figure 50. Schematic of QD activation of bR photoresponse via photonic and FRET methods.

As a bR activation source, whether through photon emission or electron transfer mechanisms, the QDs are likely only able to activate bR molecules in their immediate vicinity. Additionally, since this platform is being designed as a sensing system, it is assumed that the target molecule will only be able to interact with the outer-most layers of the hybrid platform. In the proposed system of utilizing functionalized QDs as the target binding substrate, it will be ideal to apply a single monolayer of QD's on the surface of a bR electrode in addition to maltose binding protein for target detection, as illustrated in Figure 51. Therefore the need for bulk PM electrodes, that can be up to 10,000 monolayers thick, is diminished. Applying a functional QD monolayer on top of a thick PM substrate would only be able to modulate the top-most PM monolayers and ultimately provide little modulation to the overall PM photovoltaic response. Other proposed sensing mechanisms, such as the fused MBP-bR hybrid being developed by Eric Winder [114] will also need to be applied on the monolayer level for the same reasons. 


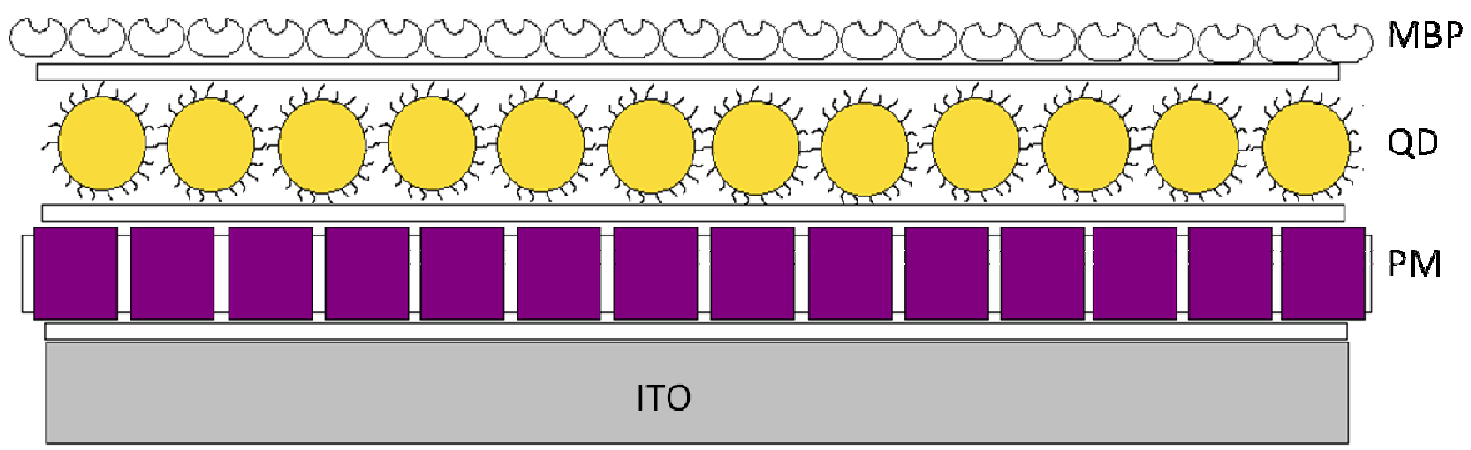

Figure 51. Schematic of nanoparticle proximity achieved through I-SAM deposition techniques.

Through the use of I-SAM techniques it is possible to engineer the deposition of materials in a layer-by-layer manner. In this work the I-SAM technique will be used to create a highly oriented and electrically active multi-monolayer bR base with the potential to easily add further materials, such as functionalized QDs, within nano-scale proximity.

\subsection{BR IONIC-SELF ASSEMBLED MONOLAYER FILMS}

The base layer of the proposed sensing system is composed of a multi-monolayer bacteriorhodopsin film. The I-SAM technique is one potential method to precisely deposit bR monolayers in a controlled fashion. It is a robust technique that allows for multiple layers to be constructed, while retaining the ability to continue layer deposition on top of the bR-base. 


\subsection{1 bR Preparation for I-SAM Deposition}

To be applicable for use with I-SAM deposition techniques bR must be adjusted to have a strong positive or negative charge. Since PM has an isoelectric point of pH 5.2 [115], it is most easily managed as a polyanionic nanomaterial by adjusting the solution $\mathrm{pH}$ above its isoelectric point, but below the $\mathrm{pH}$ level where the bR retinal is released. Previous research suggests the optimal PM solution $\mathrm{pH}$ for both I-SAM deposition and subsequent photoelectric activity is approximately $\mathrm{pH} 9.4[116,117]$. For the I-SAM electrostatic deposition of PM in these studies the PM is suspended at $0.5 \mathrm{mg} / \mathrm{ml}$ in $10 \mathrm{mM}$ borate buffer at $\mathrm{pH}$ 9.5. The sodium borate used to make the buffer is dissolved in $18 \mathrm{M} \Omega \mathrm{ddI}$ Millipore water to the desired molarity, which is then titrated to $\mathrm{pH} 9.5$ through the dropwise addition of $0.05 \mathrm{M} \mathrm{NaOH}$. As shown in Figure 52 , it was determined that the zeta potential of the prepared $\mathrm{PM}$ solution at $\mathrm{pH} 9.5$ is predominately negative. The zeta potential is a measure of the material's electric potential at the surface-water interface. Although Figure 52 shows a wide range of zeta potential values, the mean zeta potential was determined to be approximately $-25.45 \mathrm{mV}$. By I-SAM standards this is considered to be a moderate electric potential, but is sufficient for PM fragment adsorption to a polycationic surface.

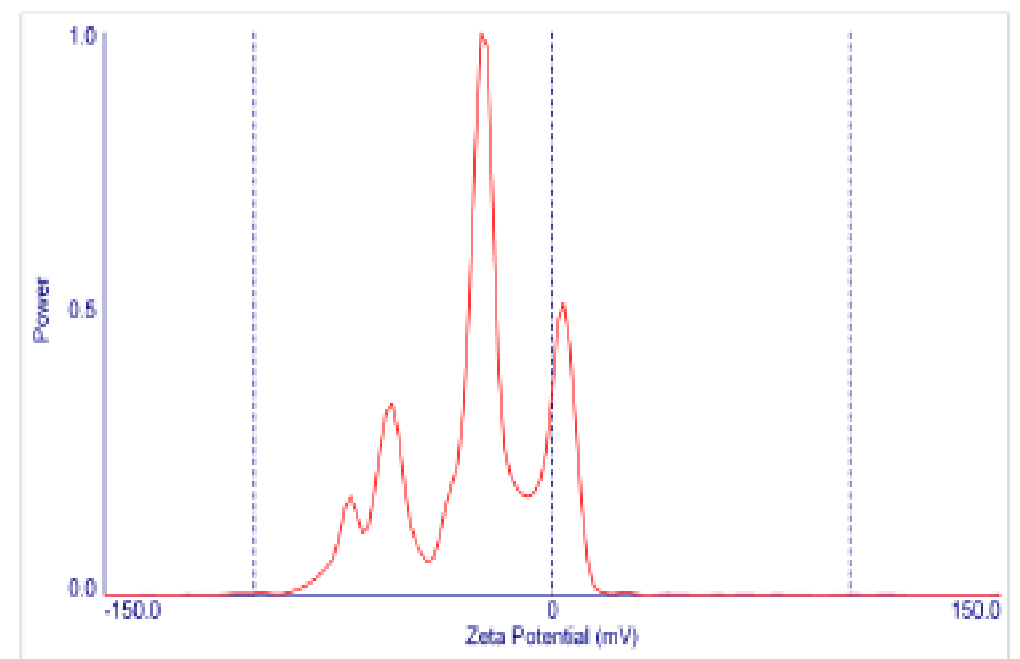

Figure 52. Zeta potential analysis of PM fragments at $\mathrm{pH} 9.5$ showing an average $P M$ charge of $-30.03 \mathrm{mV}$. 
The ITO substrates $(5 \times 25 \times 0.5 \mathrm{~mm})$ upon which the I-SAM films were constructed were cleaned using standard techniques. Specifically the slides were placed in a 2:1:1 (v/v) solution of chloroform:acetone:ethanol and sonicated for 30 minutes at $25^{\circ} \mathrm{C}$. A negative surface charge was achieved by placing the cleaned ITO substrates into a $2 \% \mathrm{KOH}$ solution and agitating for 30 minutes in an ultrasonic bath. The strong $\mathrm{KOH}$ base removes protons from the ITO surface, rendering it with a net negative charge. All ITO slides were stored in Milli-Q ddI water until use. The polycationic material utilized in this work is the positive polyelectrolyte poly(dimethyldiallylammonium chloride) solution (PDAC). Specifically the low molecular weight PDAC, $\mathrm{M}_{\mathrm{w}}=100,000-$ 200,000, was purchased from Sigma Aldrich, product 409014. The PDAC solution was prepared to $2 \mathrm{mg} / \mathrm{ml}$ in $0.5 \mathrm{M} \mathrm{NaCl} \mathrm{pH} 6.8$ solution for use as the positively charged ISAM material. For use in I-SAM deposition the PM is applied at a solution concentration of $0.5 \mathrm{mg} / \mathrm{ml}$. Example solutions are shown in Figure 53.

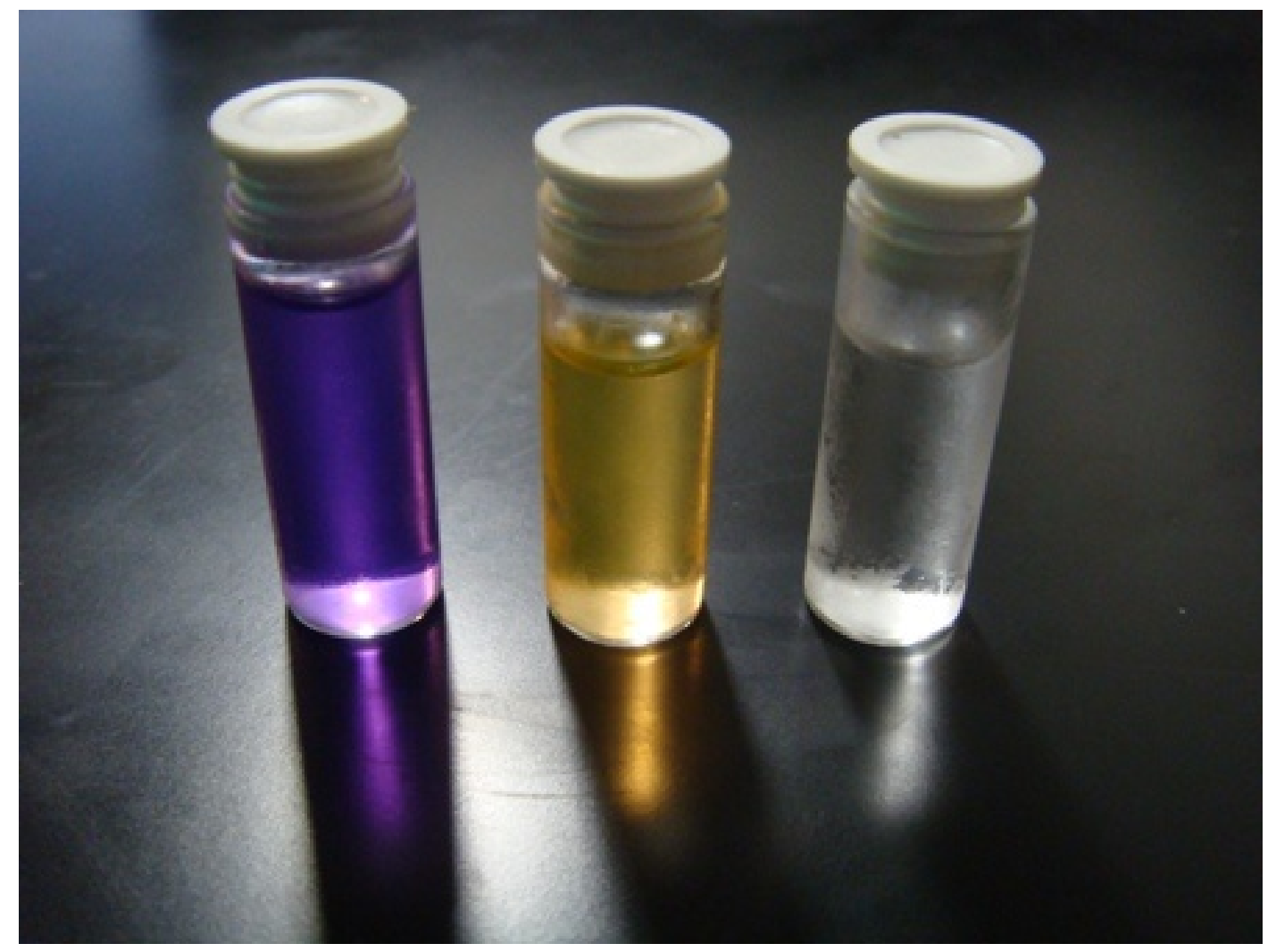

Figure 53. Materials used for I-SAM deposition. From left to right: PM, QD, PDAC. 
The dipping mechanism of a Langmuir-Blodgett trough (KSV-2000) was used to expose the ITO substrate in a stable/consistent manner to the desired adsorbing material. The monolayers were assembled by submerging the ITO slide into the desired solution for a set duration to allow for charged material adsorption. Previous work has shown that the optimal deposition time to achieve a single monolayer of PM is 5 minutes [82]. Likewise, a deposition duration of 5 minutes was used for the PDAC as well. Each adsorption period was followed by a thorough rinse in $\mathrm{pH} 9.4$ Milli-Q ddI and the substrate was dried with nitrogen. The absorption properties of the bR I-SAM films were measured on a Perkin-Elmer Lambda 950 UV/VIS/NIR spectrometer. Topography measurements were performed with a Veeco CP-II atomic force microscope (AFM).

\subsection{2 bR I-SAM Film Physical Properties}

To ensure single monolayer adsorption, I-SAM films of a single bR/PDAC bilayer were topographically compared to blank ITO. A Veeco CP-II atomic force microscope (AFM) was used to perform sample analysis. A blank ITO slide was initially analyzed and the AFM topography images in both 3-D and top-view formats are shown in Figure 54 and Figure 55, respectively. A monolayer of PDAC and then $\mathrm{bR}$ was deposited onto the blank ITO according to the aforementioned deposition techniques. A sample AFM

topography image in both 3-D and top-view formats are shown of the bR monolayer in Figure 56 and Figure 57, respectively. 


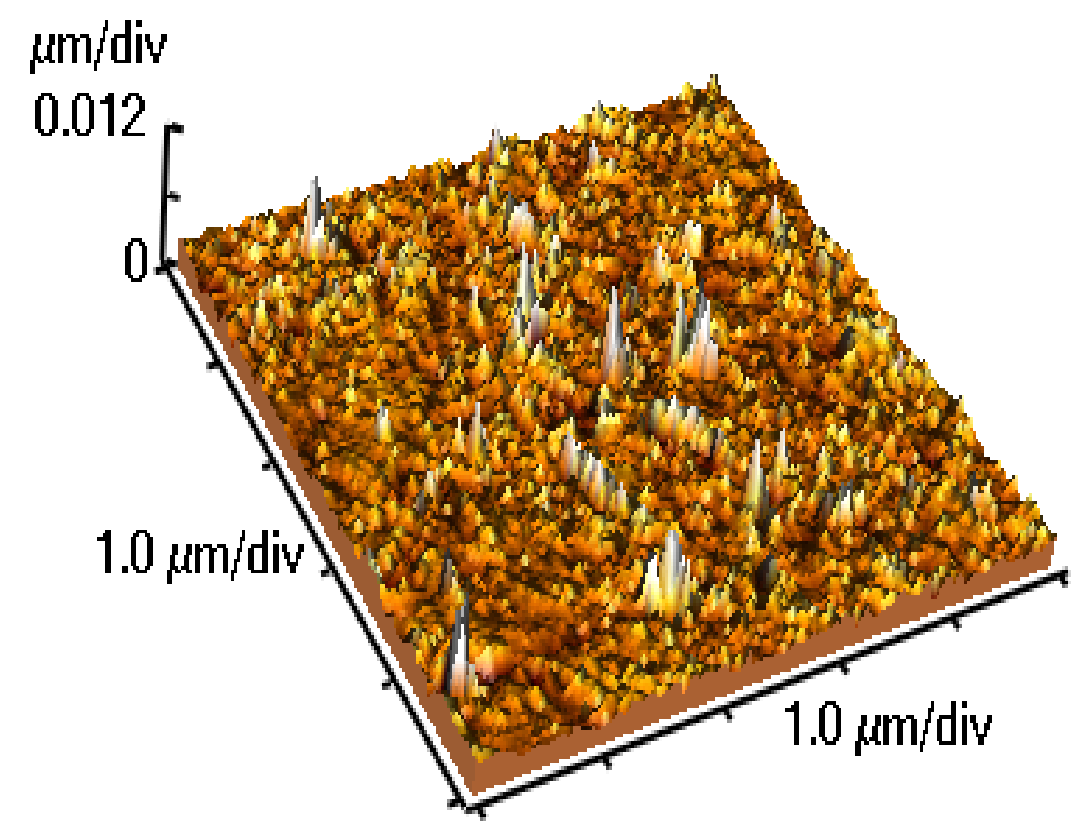

Figure 54. 3-dimensional AFM topography image of blank ITO used for PM deposition.

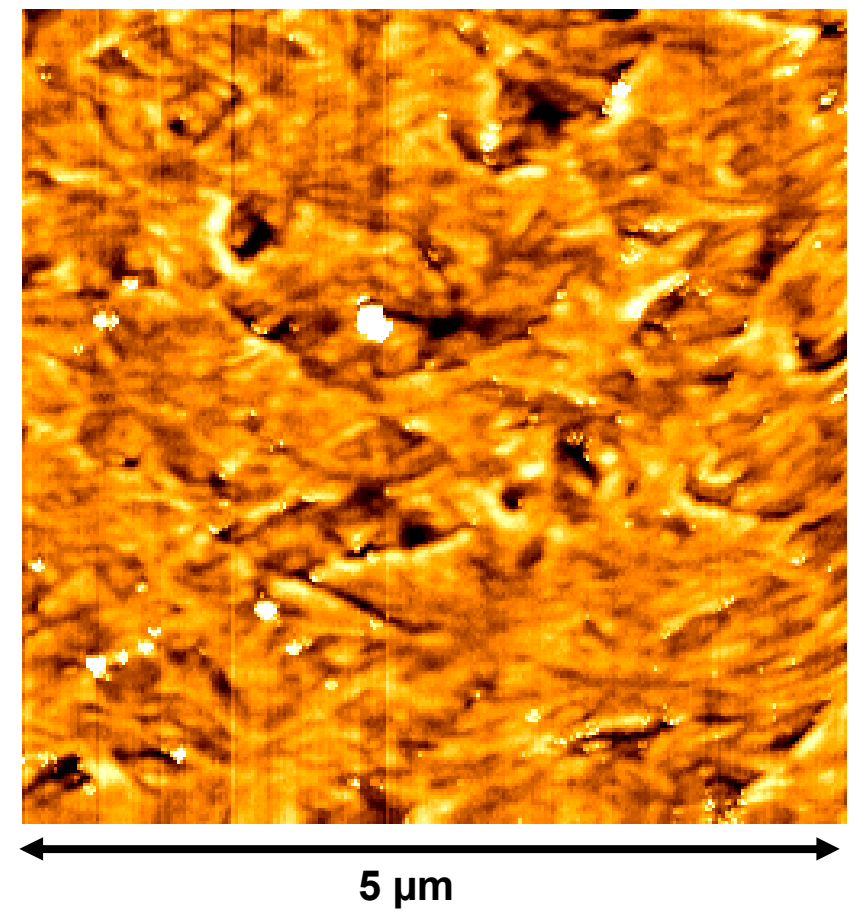

Figure 55. AFM topography image of blank ITO slide used for PM deposition. 


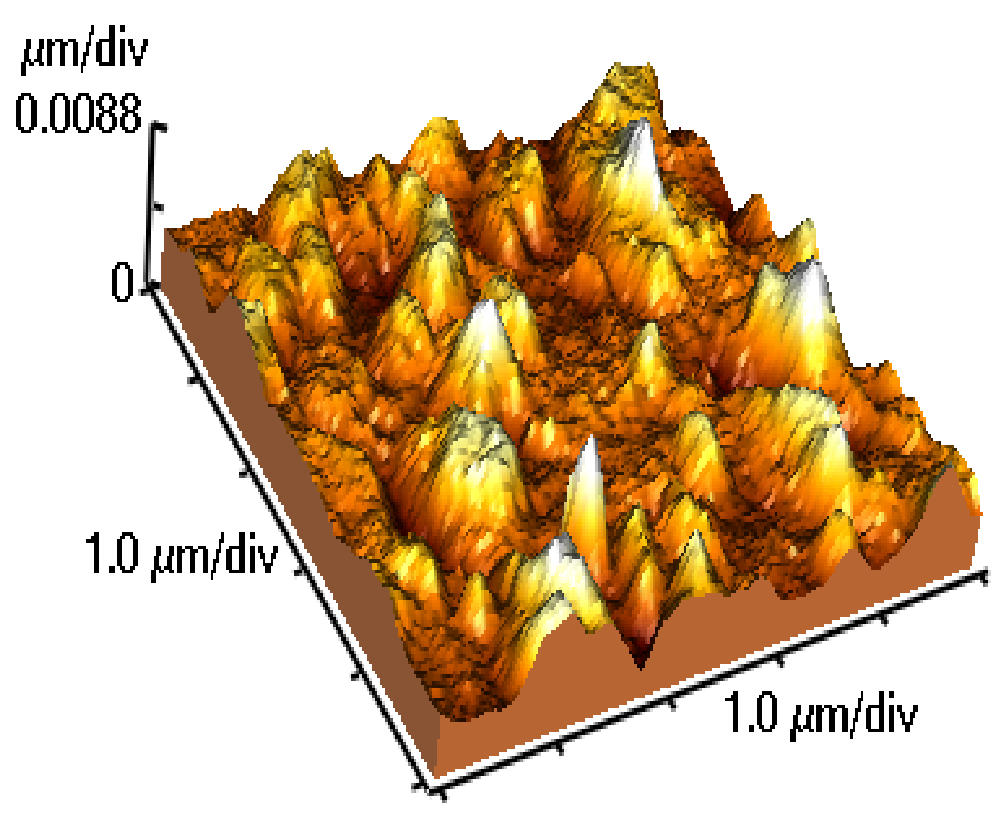

Figure 56. 3-dimensional AFM topography image of 1 PM monolayer deposited on PDAC.

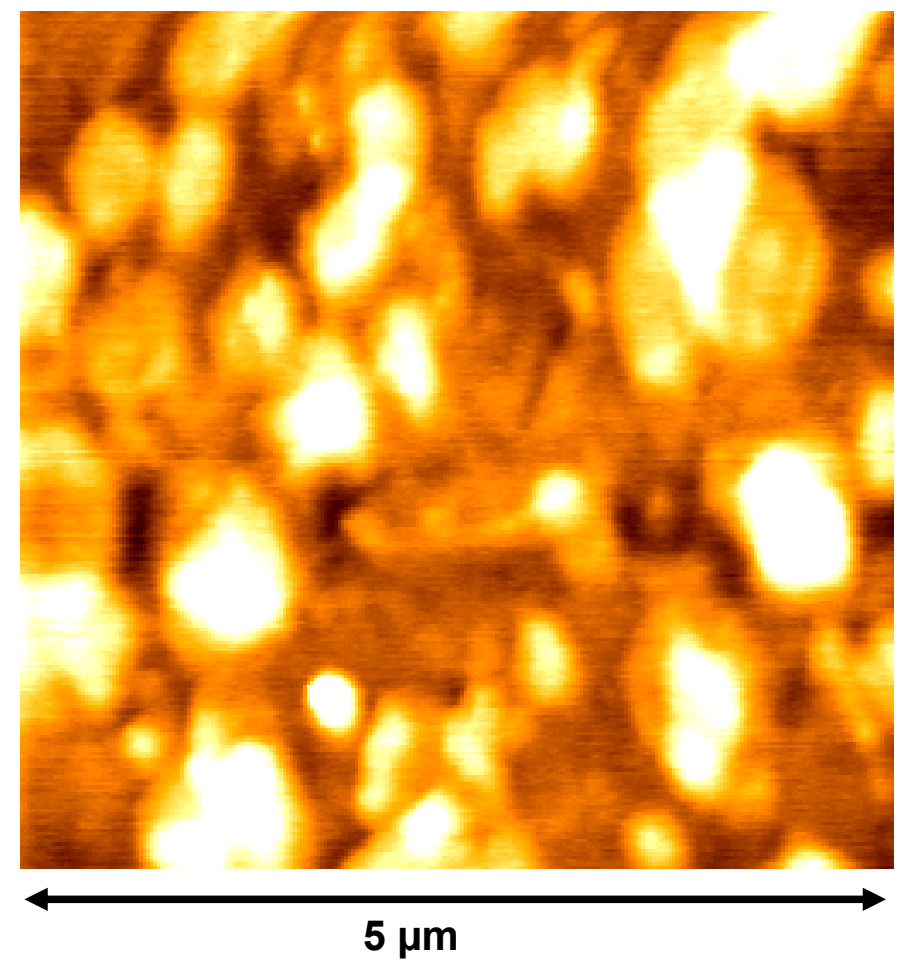

Figure 57. AFM topography scan of of 1 PM monolayer deposited on PDAC. 
The 3-D AFM topography in Figure 54 shows the flat surface of a cleaned ITO slide. On top of this slide, a monolayer of PDAC followed by a monolayer of PM was deposited, as shown in Figure 56. The 3-D AFM topography of the deposited PM clearly shows individual membrane fragments. As shown in Figure 58, AFM images confirmed the absorption of single PM monolayer patches which corresponds to the published PM thickness of $5.5 \mathrm{~nm}$. Certain sections display membrane overlap with thicknesses around $11 \mathrm{~nm}$ which has been observed in previous studies [82]. Additionally the AFM height profile shown in Figure 58 shows the width of the PM fragments to be close to the known value of $500 \mathrm{~nm}$. This shows the capability of the I-SAM technique to deposit a single monolayer of PM.
Ra: $5.695 \mathrm{~nm}$
Rq: $2.043 \mathrm{~nm}$
Height Profile
Rp: $5.116 \mathrm{~nm}$
Rv: $-3.078 \mathrm{~nm}$

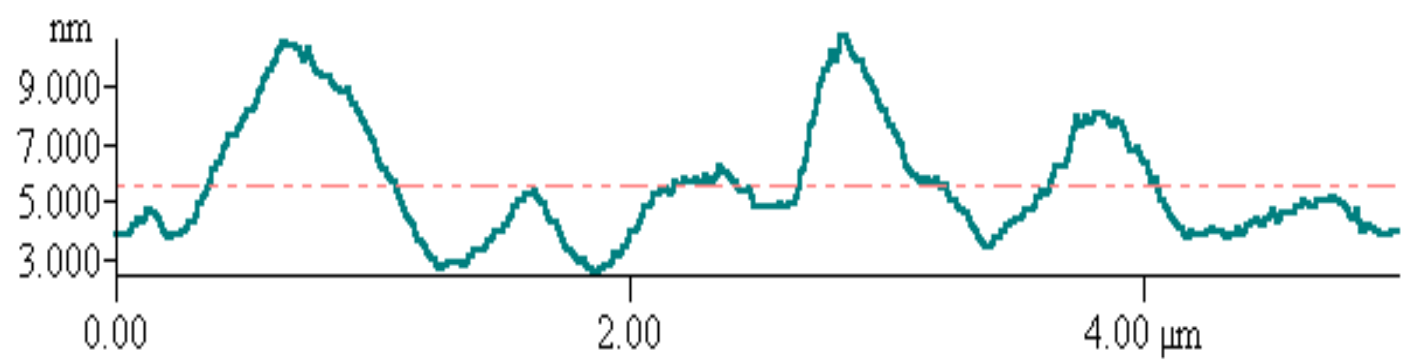

Figure 58. AFM height profile of 1 PM monolayer deposited on PDAC.

To ensure no contribution of PDAC to the measured bR 570nm absorption peak, the absorption spectra of a single PDAC monolayer was examined and is displayed in Figure 59. The PDAC absorption spectrum shows that no significant absorbance is present in the 570nm region. The PDAC monolayer does, however, absorb strongly in the sub $350 \mathrm{~nm}$ and thus might disrupt measurements of the protein $280 \mathrm{~nm}$ absorption peak. Utilizing the positively charged PDAC and negatively charged PM, the muli-layered film is electrostatically grown as shown in Figure 60. 


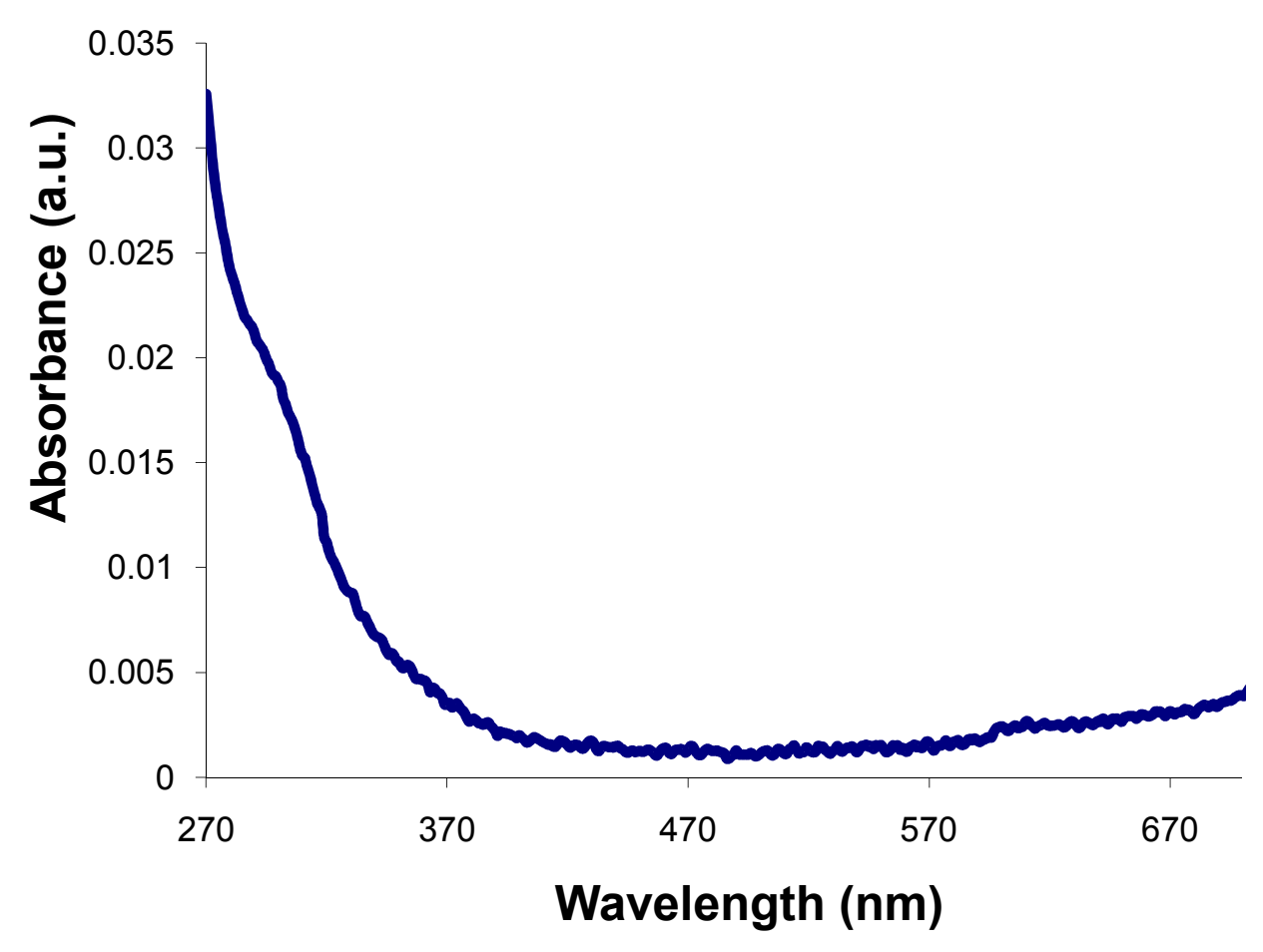

Figure 59. Absorbance spectra of PDAC in $0.5 \mathrm{M} \mathrm{NaCL}$ at pH 6.8

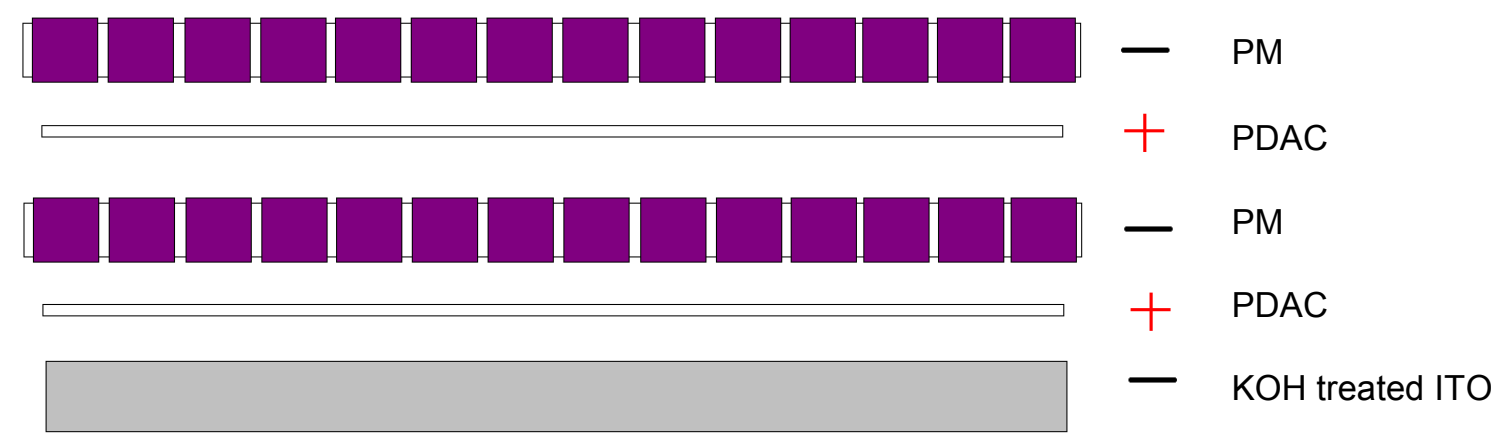

Figure 60. Layering process to create bR/PDAC multi-layered monolayer films.

The bR/PDAC bilayer assembly was monitored by the $570 \mathrm{~nm}$ absorption peak of the bR retinal and demonstrated a consistent increase in retinal absorption upon each bR monolayer addition. The absorption spectra tracking the $\mathrm{bR}$ monolayer film growth is shown in Figure 61. After each monolayer of bR was assembled on top of the previous $\mathrm{bR} / \mathrm{PDAC}$ bi-layer the absorbance spectra of the entire film was measured. It can be seen 
that each monolayer of $b R$ contributes to an increase in the typical $b R$ absorbance profile. Further analysis of the absorbance maxima at 570nm, as shown in Figure 62, demonstrates a linear trend in the absorbance increase. The linear increase in absorbance as each bR layer was deposited reveals that a stable and consistent multi-layered film growth was achieved.

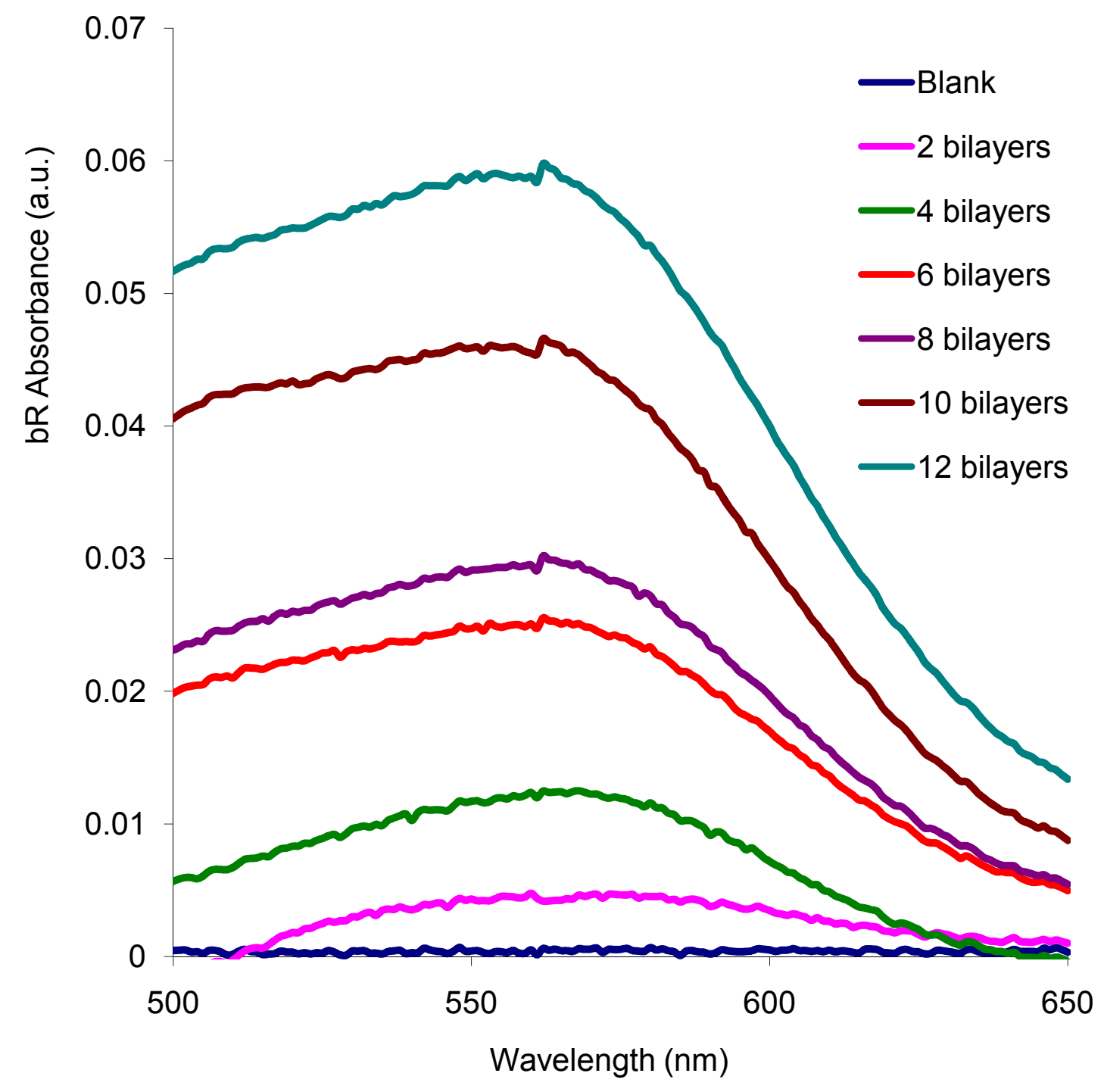

Figure 61. bR absorbance spectra for select bR/PDAC bilayers as it is assembled. Bilayers constructed on negatively charded ITO with PDAC (+) and bR (-) being alternately deposited. 


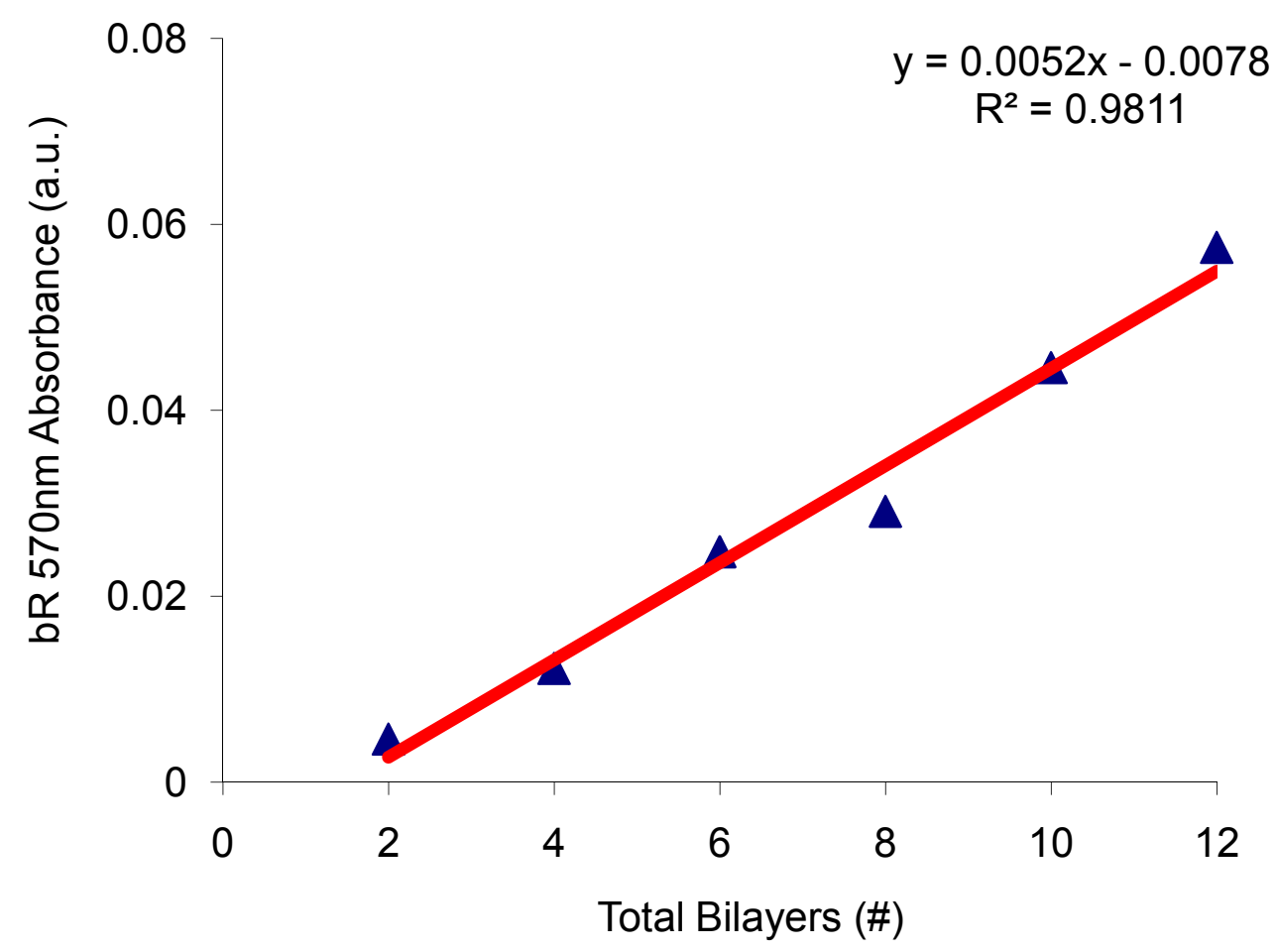

Figure 62. The $b R_{570} \mathrm{~nm}$ absorption peaks for consecutively deposited $b R / P D A C$ bi-layers.

\section{$\underline{\text { 5.1.3 bR I-SAM Film Electrical Activity }}$}

With succesful deposition of bR monolayers using the I-SAM technique, the photovoltaic properties of the created electrode was tested. With monolayer film PM electrodes it proved difficult to press a counter ITO electrode on top of the PM electrode without creating a short-circuit. This was possible, however, with the thicker 12 monolayer PM films. The current and voltage responses of the 12 monolayer PM electrode are shown in Figure 63 and Figure 64, respectively. In AC-coupled measuring mode, the light illumination yielded output magnitudes of approximately $60 \mathrm{mV}$ and $0.9 \mathrm{nA}$, thus confirming the electrical functionality of the as-created PM I-SAM films. 


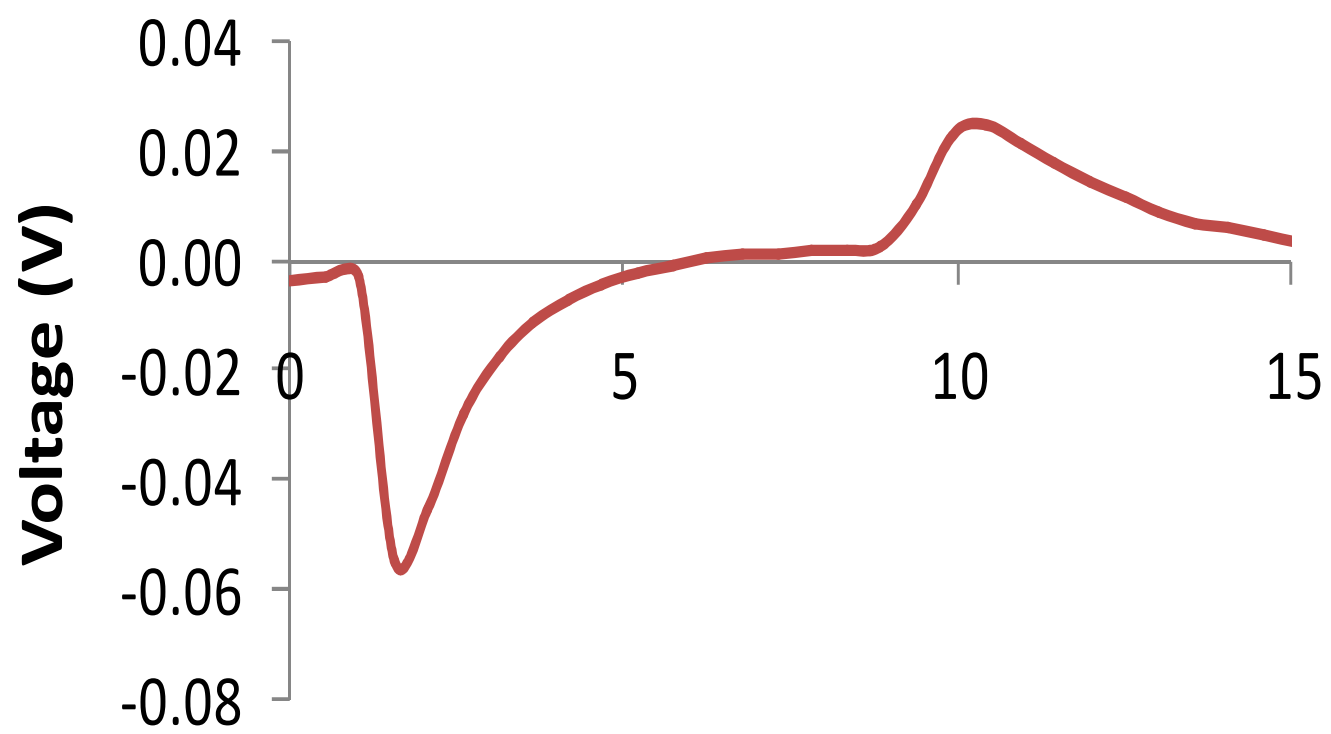

Time (s)

Figure 63. Photovoltaic response of a 12 monolayer bR I-SAM film (10mW halogen light source).

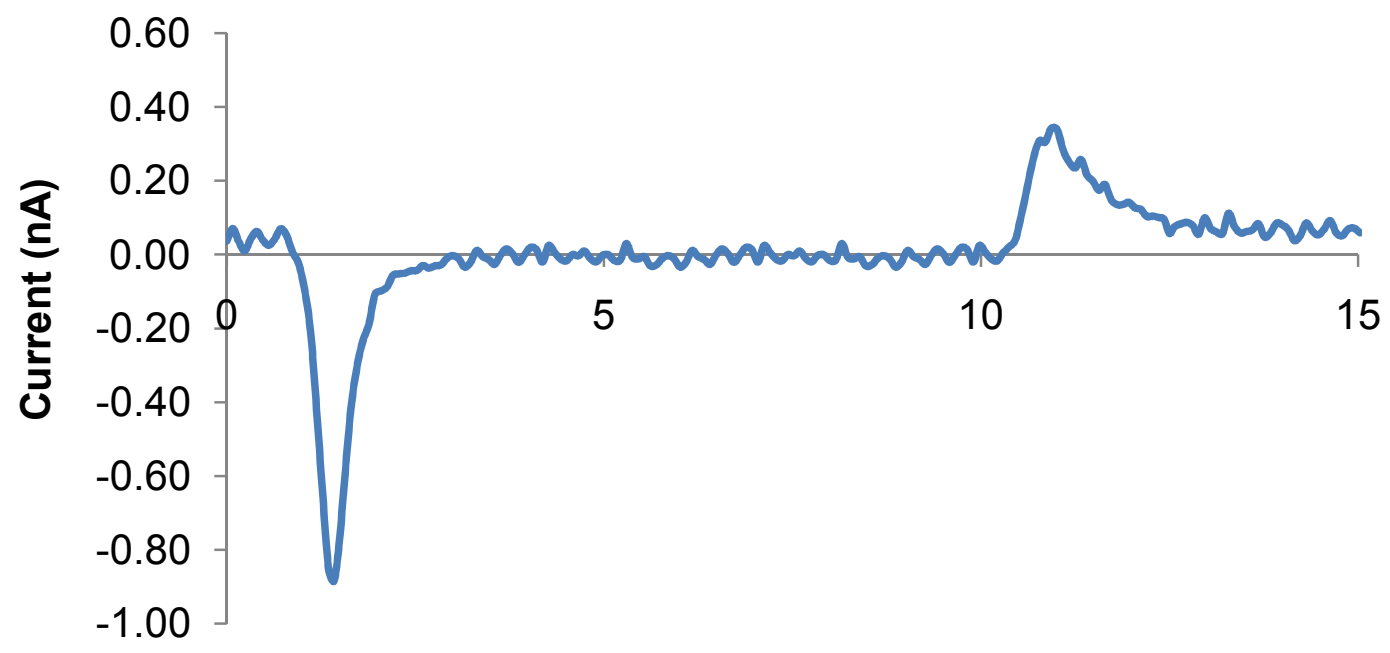

Time (s)

Figure 64. Photocurrent response of a 12 monolayer bR I-SAM film (10mW halogen light source). 
To measure the photovoltaic activity of bR electrodes thinner than 12 monolayers, a new electrical setup was used. Instead of clamping an ITO counter electrode directly on the PM surface, a potassium chloride $(\mathrm{KCl})$ electrolyte spacer layer was integrated. Specifically a $1 \mathrm{~mm}$ thick $\mathrm{x} 4 \mathrm{~mm}$ diameter o-ring was placed on top of the bR monolayer electrode. The o-ring was filled with approximately $20 \mu \mathrm{L}$ of $0.5 \mathrm{M} \mathrm{KCL}$ solution and a top ITO electrode was placed on top of the o-ring. The electrode setup was clamped together to form an approximate $1 \mathrm{~mm}$ separation distance between the $\mathrm{bR}$ and counter electrode. Electrical measurements were performed on a Keithley-4200 semiconductor parameter analyzer.

A comparison of the photovoltaic output of 3 monolayer and 6 monolayer bR films is shown in Figure 65. The 3 monolayer bR film generated a steady-state photovoltage maximum of $2.72 \mathrm{mV}$, while the 6 monolayer bR film produced an output of $5.82 \mathrm{mV}$. The 6 monolayer film produced an output that is 2.14 times larger than the 3 monolayer films, which roughly correlates to the thickness difference in the electrodes.

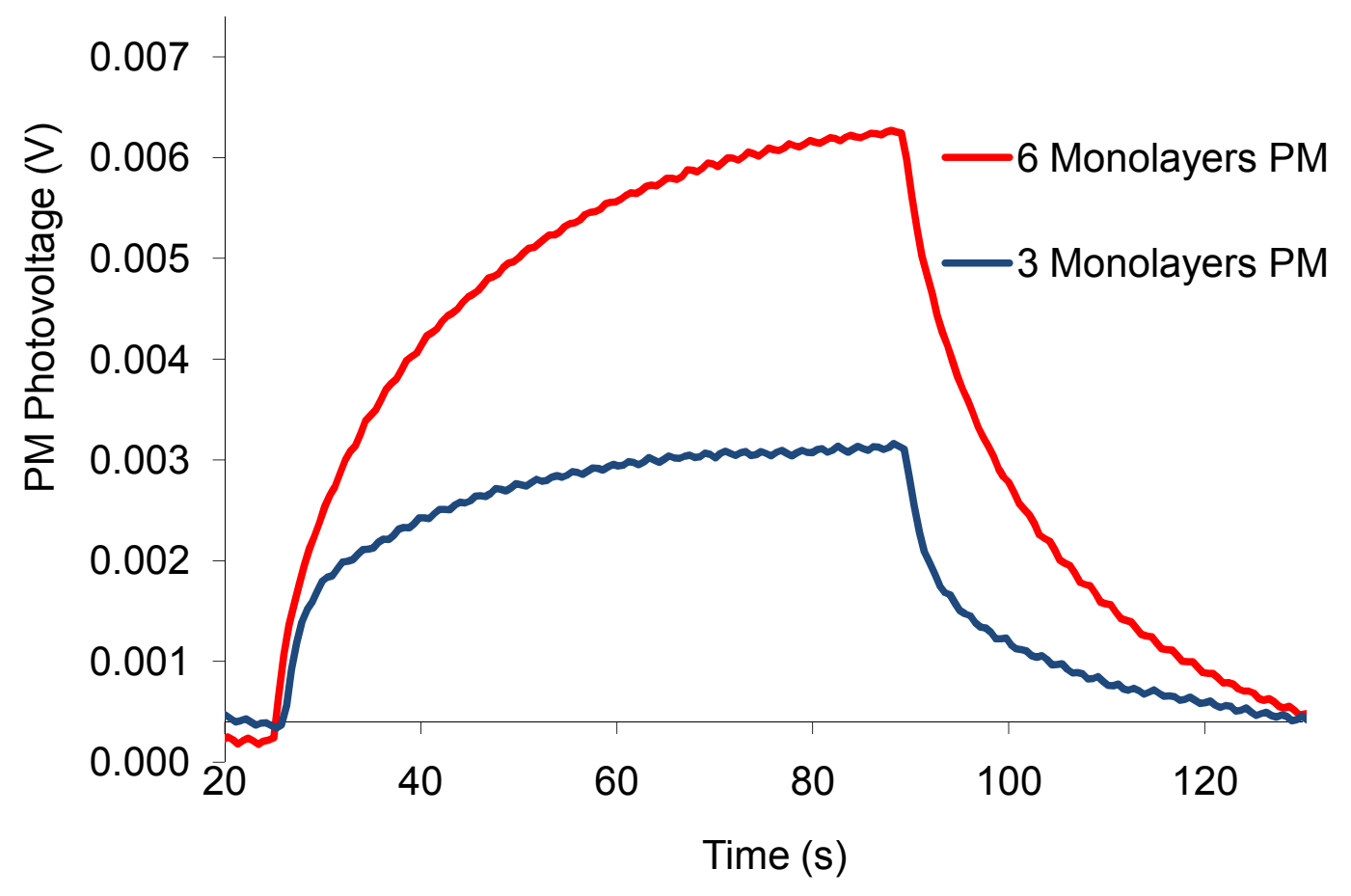

Figure 65. Comparison of 3 monolayer and 6 monolayer PM I-SAM film photovoltaic output. 
Although the photovoltaic outputs of the thin bR films was relatively small, it was still capable of producing a measurable voltage change upon variation in incident light intensity. The light sensitivity of a 3 monolayer bR film is shown in Figure 66.

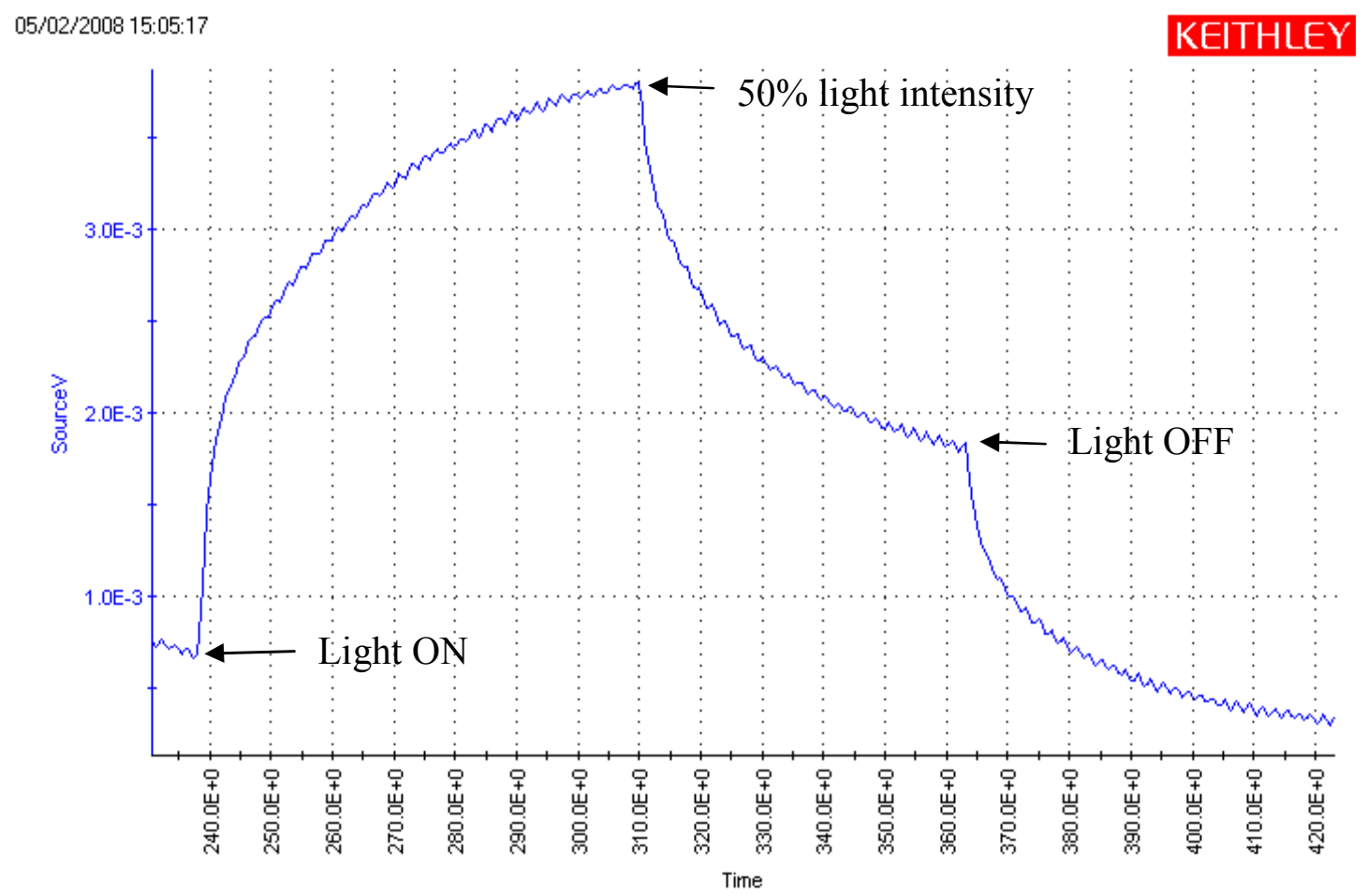

Figure 66. Effect of varying light intensity on 3 monolayer PM photovoltaic output.

\subsection{QD IONIC-SELF ASSEMBLED MONOLAYER FILMS}

The QD monolayer film will need to be integrated into sub-10nm proximity with the bR base monolayer electrode in order to facilitate potential energy transfer. Prior to integration with $b R$, it is necessary to confirm that consistent QD layers can be achieved without degrading the QD emission properties. 


\subsubsection{QD Surface Functionalization}

To be applicable for use with I-SAM deposition techniques QDs must be prepared to have a strong positive or negative charge. The primary method for imparting negative charge on a QD involves replacing the QD surface ligand with a carboxylic acid containing molecule, such as TGA [95]. A subsequent positive charge can be added by encapsulating the negatively charged QD with an amine containing molecule, such as PAA [92]. Since the negatively charged QDs are inherently smaller in diameter, they were ideal for this work to minimize the bR-QD separation distance. Thioglycolic acid capped $\mathrm{CdSe} / \mathrm{ZnS}$ QDs were obtained from Dr. Roger LeBlanc at the University of Miami. The absorbance and emission spectra of the prepared TGA-capped QDs is shown in Figure 67.

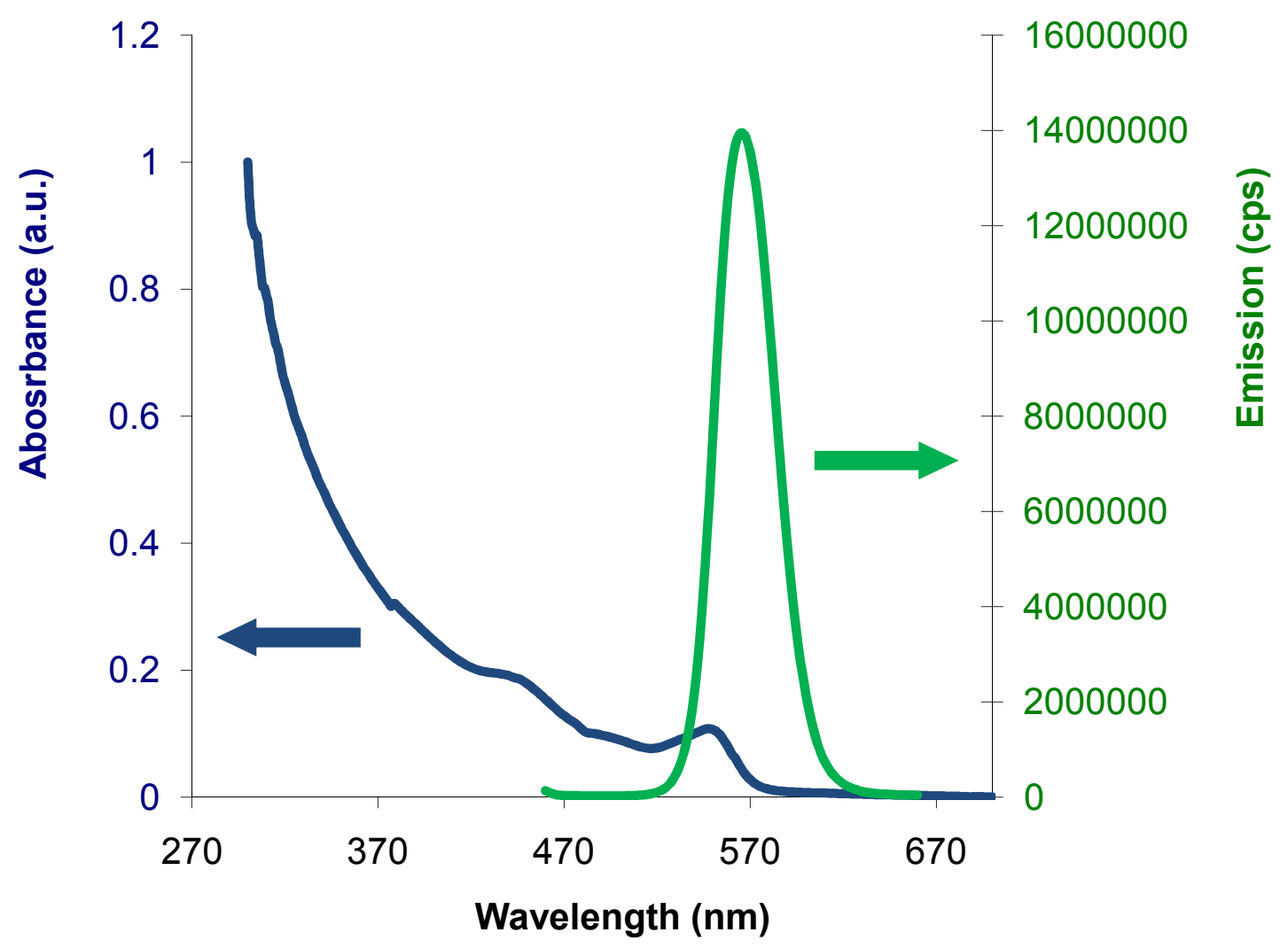

Figure 67. Emission and absorbance spectra of TGA-capped CdSe/ZnS quantum dots. 
As with the PM, the carboxyl group must be deprotonated to attain the negative charge. Therefore the TGA-capped QDs were suspended at $6.2 \mu \mathrm{M}$ in a $10 \mathrm{mM}$ sodium borate buffer adjusted to $\mathrm{pH}$ 9.5. The sodium borate used to make the buffer is dissolved in $18 \mathrm{M} \Omega$ distilled deionized (ddI) Millipore water to the desired molarity, which is then titrated to $\mathrm{pH} 9.5$ through the drop-wise addition of $0.05 \mathrm{M} \mathrm{NaOH}$. According to literature, this will yield a QD zeta potential of approximately $-29.5 \mathrm{mV}$ [95].

To create a multi-layered QD film, as depicted in Figure 68, the positively charged PDAC polymer was used as the adhesion spacer layer. The PDAC was prepared to $2 \mathrm{mg} / \mathrm{ml}$ in $0.5 \mathrm{M} \mathrm{NaCl}$ solution, adjusted to $\mathrm{pH} 6.8$. The $\mathrm{KOH}$ treated, negatively charged, ITO slides are submerged in the PDAC solution for 5 minutes to allow for the absorption of a single monolayer of the positive polymer. The excess PDAC was removed with a 2 minute rinse in $\mathrm{pH} 6.8 \mathrm{ddI}$ water, following by thorough drying with $\mathrm{N}_{2}$ gas. The slide was then submerged in the prepared QDs for 10 minutes followed by a similar rinsing step. This process was repeated until the desired number of layers was achieved.
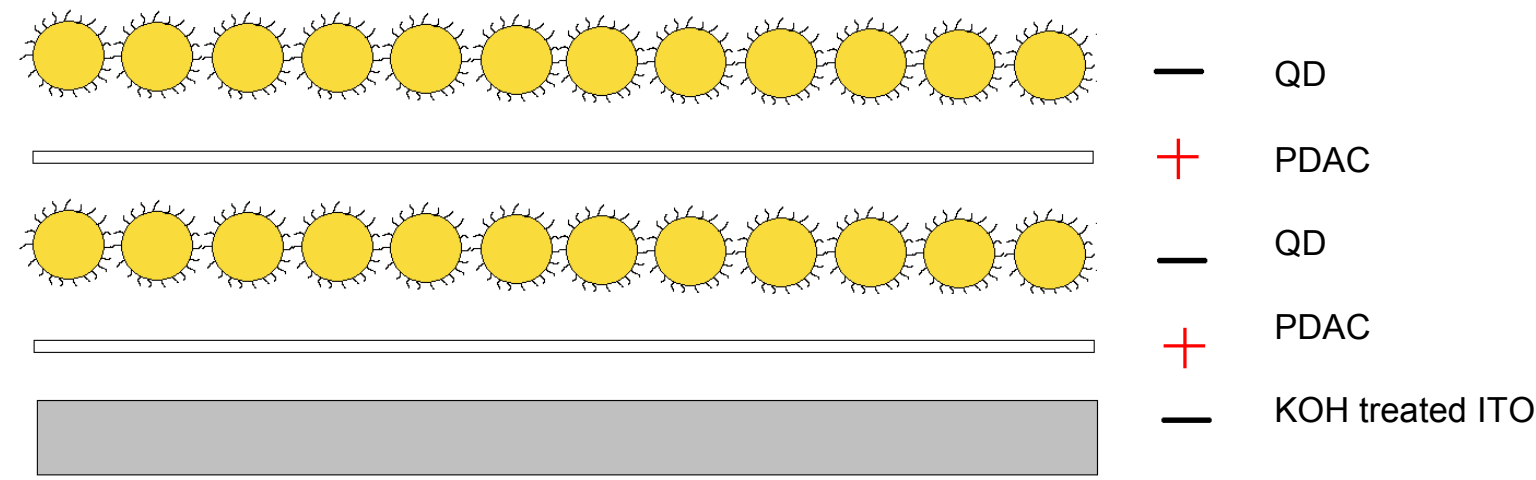

Figure 68. Layering process to create QD/PDAC multi-layered monolayer films. 


\subsubsection{QD I-SAM Film Physical and Optical Properties}

At each level of QD monolayer deposition the QD emission was measured with a Horiba Jobin Yvon SPEX Fluoromax 2 spectrofluorimeter. A modified cuvette was created to hold the ITO slides in a consistent position and angle for each measurement, as a small change in slide position has a large effect in the measured QD intensity. The spectrofluorimeter is allowed to warm up for 30 minutes prior to measurement to avoid fluctuations in excitation light intensity. All measurements were taken using a $400 \mathrm{~nm}$ excitation wavelength and $5 \mathrm{~nm}$ excitation and emission slit widths. The resulting QD emission for each deposited monolayer is given in Figure 69. A trace of the $\mathrm{QD}_{570 \mathrm{~nm}}$ emission peak for each deposited monolayer is shown in Figure 70.

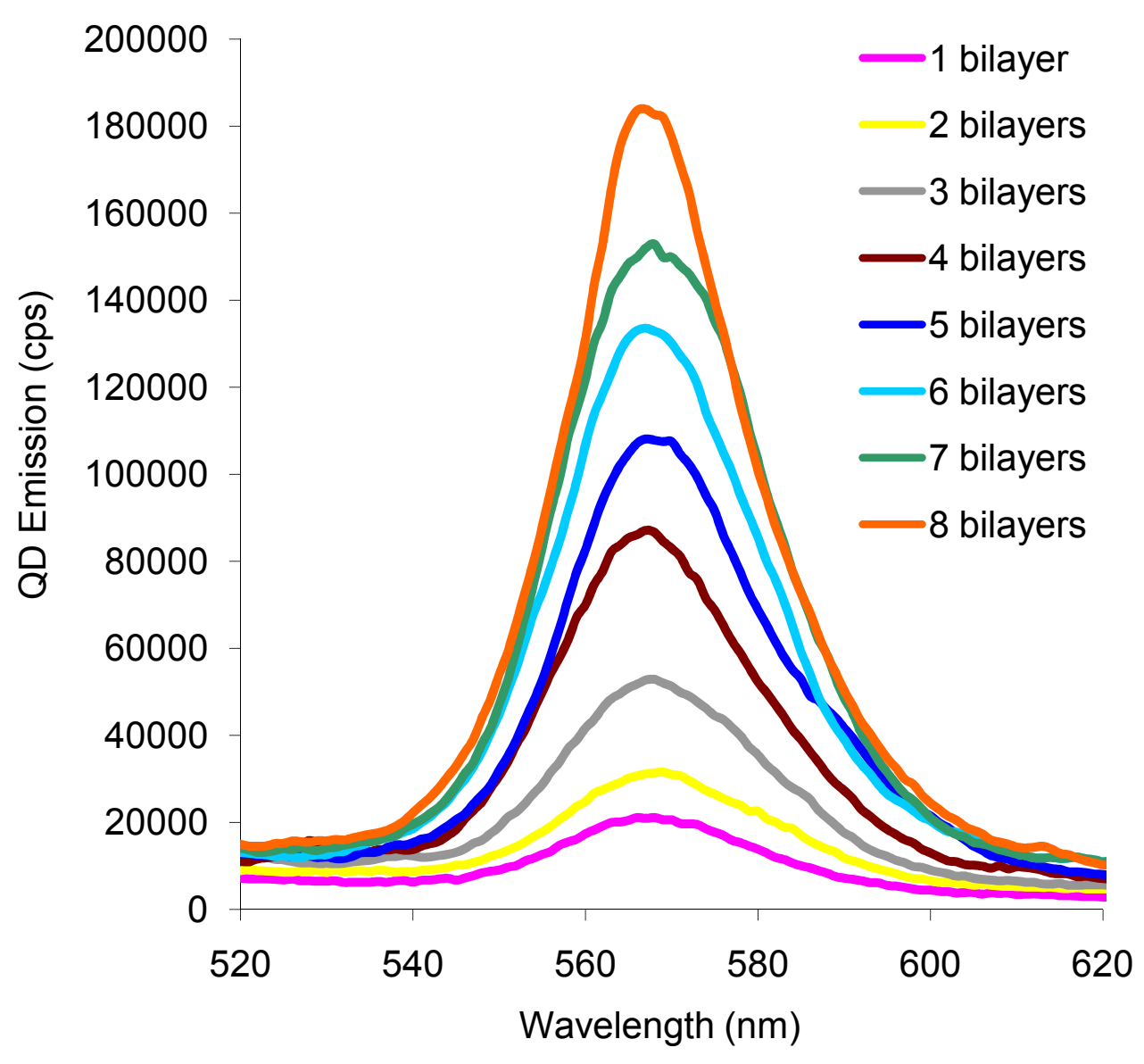

Figure 69. QD emission tracking QD/PDAC bilayer deposition. 


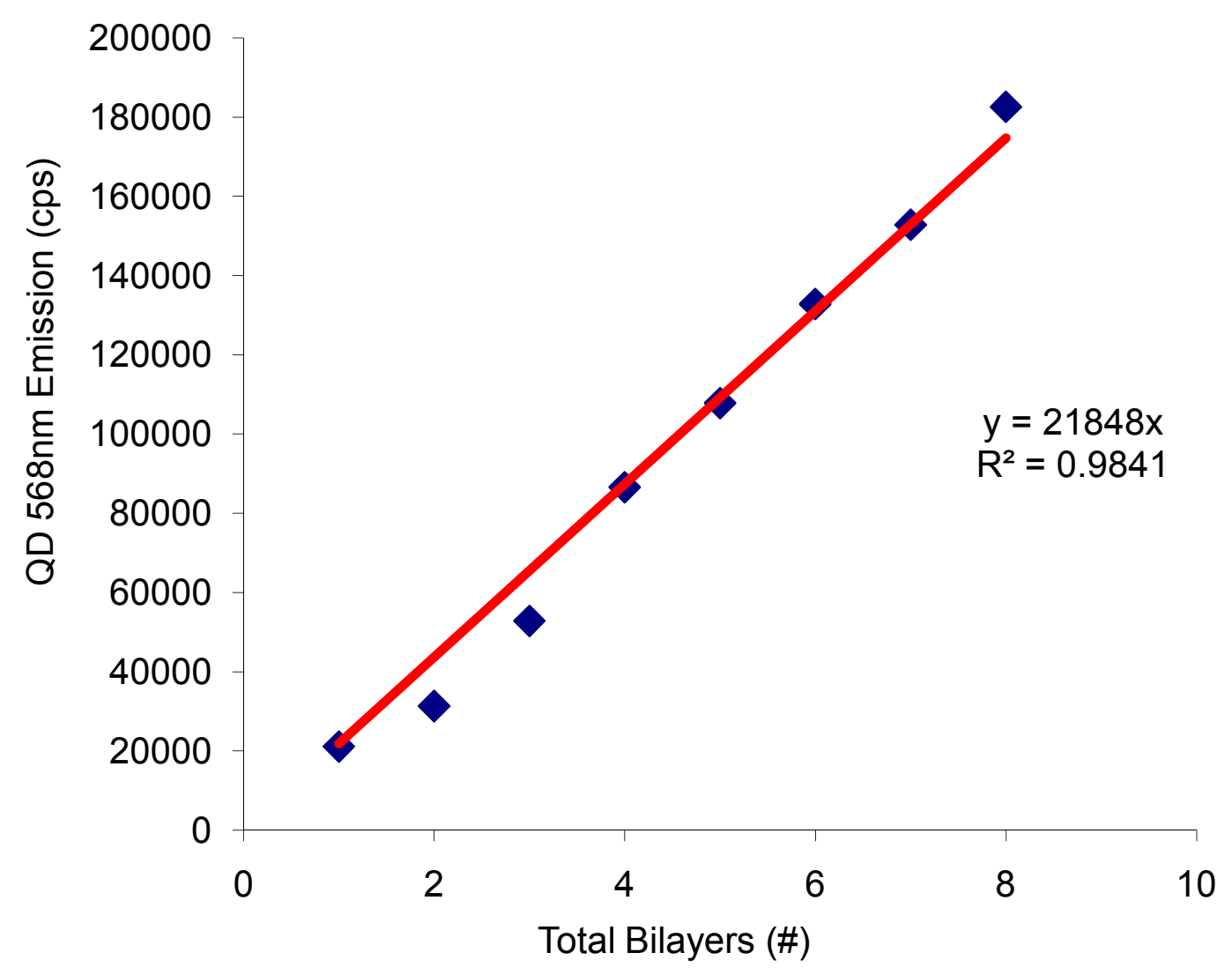

Figure 70. $Q D_{568 \mathrm{~nm}}$ emission maximum tracked through mulit-layered QD deposition.

It can be seen in Figure 69 and Figure 70 that consistent QD deposition is achieved up to 8 layers. Plotting of the emission maxima reveals that each TGA-QD layer contributes approximately $21,848 \mathrm{cps}$ to the overall emission for the given spectrofluorimeter settings.

To ensure QD absorption was due only to electrostatic attraction to an oppositely charged surface, the absorption affinity of TGA-QDs to negatively charged surfaces was also evaluated. In Figure 71 a comparison of negatively charged TGA-QD absorption affinities to positively and negatively charged surfaces is shown. The three surfaces tested in Figure 71 are negatively charged-KOH treated ITO, positively charged PDAC, and negatively charged polystyrene sulfonate (PSS). The PDAC surface was prepared by dipping a $\mathrm{KOH}$ treated ITO slide into a $2 \mathrm{mg} / \mathrm{ml}$ PDAC solution, $0.5 \mathrm{M} \mathrm{NaCl} \mathrm{pH} 6.8$, for 5 minutes. Likewise the PSS surface was prepared by dipping a PDAC-coated ITO 
slide into a $2 \mathrm{mg} / \mathrm{ml}$ PSS solution, $0.5 \mathrm{M} \mathrm{NaCl} \mathrm{pH}$ 9.0, for 5 minutes. All prepared slides were rinsed and dried with $\mathrm{N}_{2}$ gas prior to TGA-QD absorption. Once the base substrates were prepared the slides were immersed in a $6.2 \mu \mathrm{M}$ solution of TGA-QDs, $10 \mathrm{mM}$ borate $\mathrm{pH}$ 9.4, for 10 minutes. All slides were rinsed and dried with $\mathrm{N}_{2}$ gas prior to fluorospectrometer measurements.

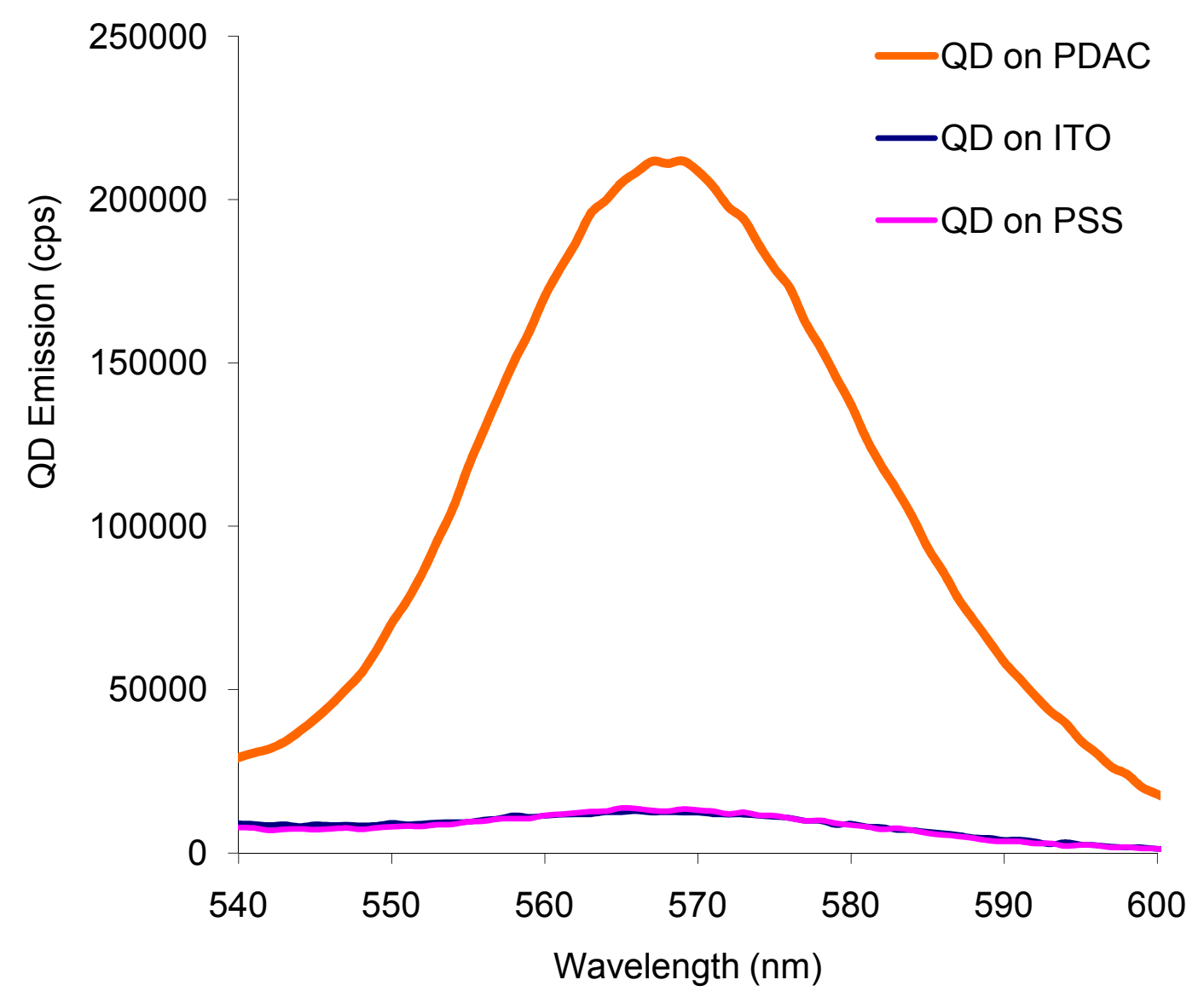

Figure 71. QD emission comparison when deposited on differently charged substrates.

It can be seen that the negatively charged TGA-QDs preferentially absorb to the positively charged PDAC material. Conversely, barely any TGA-QD absorption was present on either negatively charged ITO or the negatively charged PSS layer. Absorption of TGA-QDs to PDAC yielded a QD photonic output maximum at 568nm of $211,000 \mathrm{cps}$, compared to a $568 \mathrm{~nm}$ emission maximum of $11,000 \mathrm{cps}$ for the ITO and PSS 
substrates. Results show that a small amount of TGA-QDs will absorb onto the negatively charged substrate, however that magnitude of such absorption is nearly 200 times less than that of a positively charged PDAC substrate. It should be noted that the fluorospectrometer settings were altered between Figure 71 and Figure 69 to amplify the single QD layer spectra, and thus the QD emission cannot be compared between the two studies.

\subsection{QD-BR HYBRID IONIC-SELF ASSEMBLED MONOLAYER FILMS}

Integrating bR and QDs nanomaterials into multi-layered I-SAM films will allow for the evaluation of their nano-scale interactions. The I-SAM technique allows for QDs to be closely integrated with bR monolayer films in a controlled fashion, in addition to allowing for controlled engineering of the layering order and composition. It is a robust technique that allows for multi-material systems to be constructed on any charged substrate.

\subsubsection{QD-bR Hybrid I-SAM Film Construction}

To create a multi-layered film utilizing negatively charged QD and PM nanomaterials, as depicted in Figure 72, the positively charged PDAC polymer was used as the adhesion spacer layer. The PDAC was prepared to $2 \mathrm{mg} / \mathrm{ml}$ in $0.5 \mathrm{M} \mathrm{NaCl}$ solution, adjusted to $\mathrm{pH}$ 6.8. The KOH treated, negatively charged, ITO slides were submerged in the PDAC solution for 5 minutes to allow for the absorption of a single monolayer of the positive polymer. The excess PDAC was removed with a 2 minute rinse in $\mathrm{pH} 6.8$ ddI water, following by thorough drying with $\mathrm{N}_{2}$ gas. The slide was then submerged either in the prepared QDs for 10 minutes or prepared PM for 5 minutes, followed by a similar rinsing step. Both the PM and QDs were suspended in 10mM borate buffer at $\mathrm{pH} 9.4$ to facilitate deprotonation of the carboxyl groups and achieving a strong negative charge. 


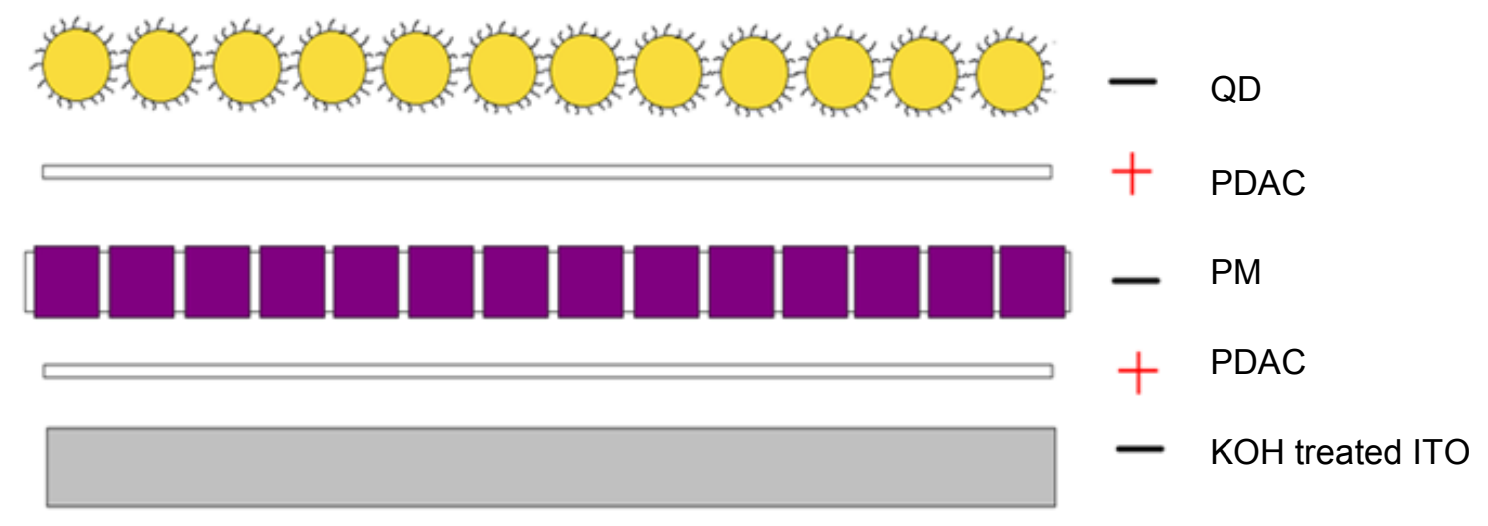

Figure 72. Layering process to create QD/PDAC/bR multi-layered monolayer films.

\subsubsection{QD-bR Hybrid I-SAM Film Physical Properties}

For this study the I-SAM films were constructed with alternating layer-by-layer absorption of bR and QDs, with PDAC as the oppositely charged spacer. Specifically it was built in a repetitive trilayer fashion, each trilayer consisting of a bR-PDAC-QD multi-layered section. Therefore, for example, the base trilayer was constructed in the order of: ITO-PDAC-bR-PDAC-QD. Subsequent trilayers were deposited in the same order, excluding the ITO. Film deposition was confirmed by monitoring the QD emission spectra, as shown in Figure 73. The QD emission maximum at each layer deposition is plotted in Figure 74 to further elucidate the layering effects on QD emission. 


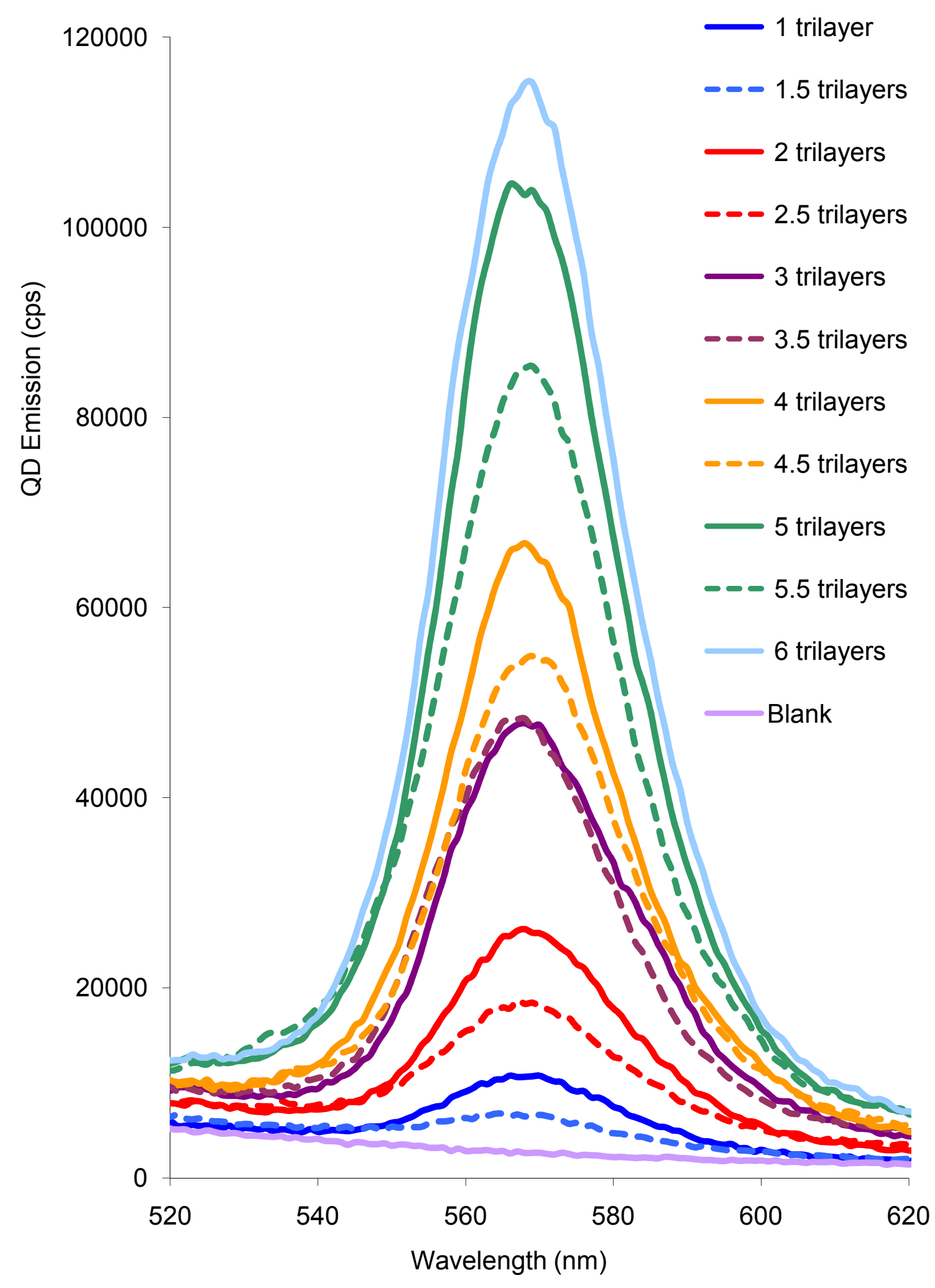

Figure 73. QD emission tracked during QD (solid lines)/PDAC/bR (dotted lines) multi-layered films. 


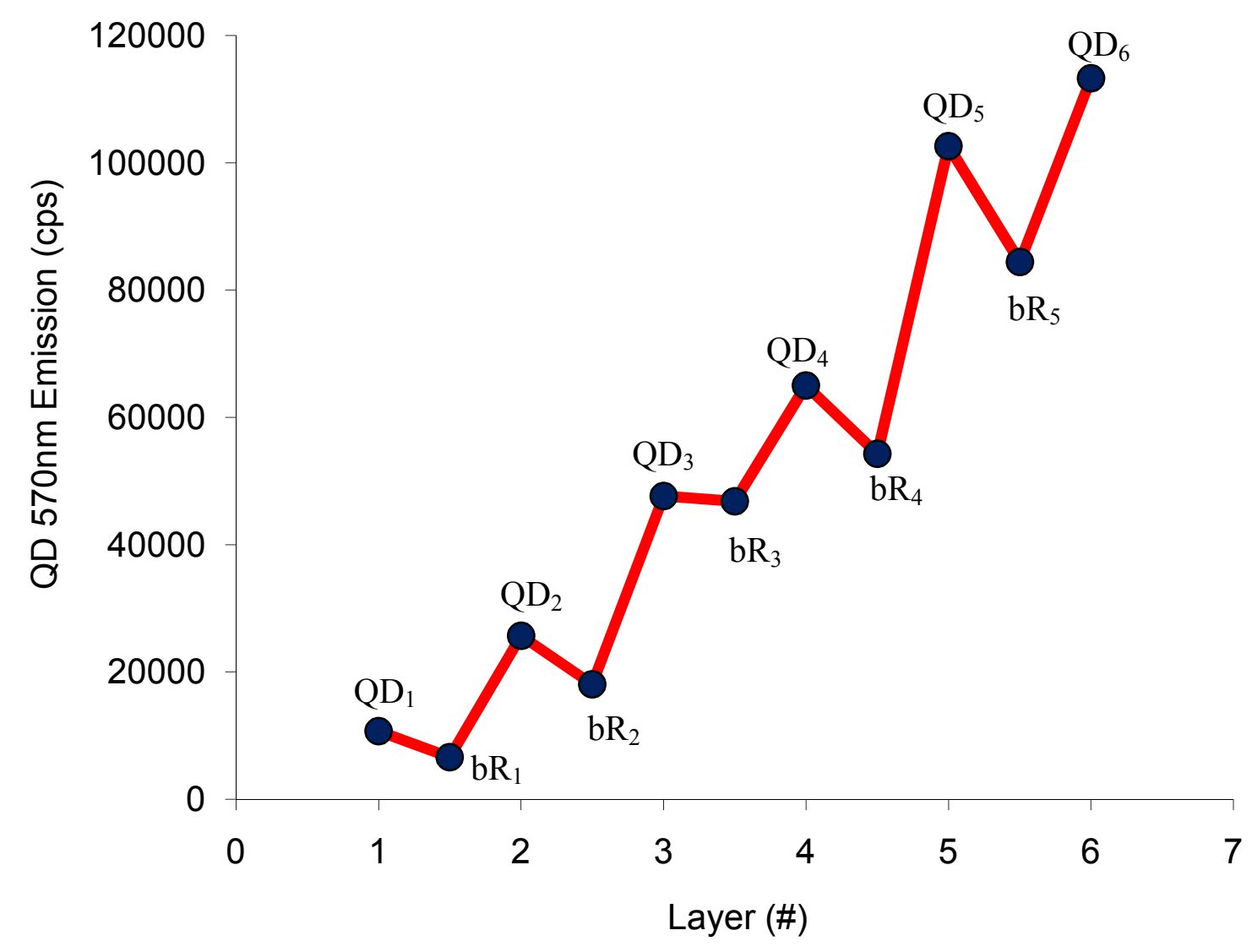

Figure 74. $\mathrm{QD}_{568 \mathrm{~nm}}$ emission maxima tracked during each QD-bR monolayer deposition.

In Figure 73 the 'half' trilayers signify where a bR layer was electrostatically deposited and the 'full' trilayers signify where QDs were layered. It can be seen that integration of bR monolayers into QD I-SAM films produces a large quenching effect on the QD emission. A more detailed view of these quenching effects is shown in Figure 74, where it can be seen that each bR layer induces a $20 \%-30 \%$ reduction, on average, of the QD $568 \mathrm{~nm}$ emission maxima. It is suggested that these quenching effects are due to photonic quenching by the bR retinal in conjunction with electron transfer from the excited QD to the bR molecule. 


\subsubsection{QD-bR Hybrid I-SAM Film Electrical Activity}

With the succesful integration of $\mathrm{bR}$ and QDs into a single multi-layered I-SAM electrode, the photovoltaic property's of the created electrode was tested. As with previous measurements on bR I-SAM films, a potassium chloride $(\mathrm{KCl})$ electrolyte was used as a conducting medium between the spaced bR electrode and opposing counter electrolde. Instead of using an o-ring spacer to contain the electrolyte and maintain electrode separation, this work utilized a specially built electrochemical cell. Specifically an optically transparent fluorospectrometer cuvette was modified to hold the $b R$ electrode, counter electrode, and $\mathrm{KCl}$ electrolyte in a controlled and consistent manner. The counter electrode in this setup was a $1 \mathrm{~mm}$ diameter platinum wire. In addition to ensuring proximity control of the electrode system, this measurement setup also allowed integration of the electrode setup into the fluorospectrometer, ultimately allowing a variety of wavelength options for $\mathrm{bR}$ or $\mathrm{QD}$ activation in conjunction with the ability to monitor QD output.

For the electrical measurements, the bR photovoltaic response was amplified with a $11 x$ inverting operational amplifier and measured on a 100 $\mathrm{MHz}$ oscilloscope. A comparison of the photovoltaic output of a 3 monolayer bR film and its subsequent output after a single QD monolayer is deposited on the surface is shown in Figure 75 and Figure 76, respectively. In Figure 75 the photovoltaic output of a 3 monolayer bR I-SAM film in response to $5 \mu \mathrm{W} / \mathrm{cm}^{2} 570 \mathrm{~nm}$ photonic illumination is shown. The system was ACcouple and yielded a photovoltaic output of approximately $0.5 \mathrm{mV}$. Using the aforementioned electrostatic deposition techniques, a PDAC layer was placed on top of the 3 monolayer bR electrode followed by QD adsorption. The bR-QD hybrid I-SAM electrode was place back into the measurement setup and reactivated with $5 \mu \mathrm{W} / \mathrm{cm}^{2}$ $570 \mathrm{~nm}$ photonic illumination. The AC-coupled output shown in Figure 76 reveals a large increase in the $\mathrm{bR}$ photovoltaic response. The magnitude of the response increased to approximately $1.5 \mathrm{mV}$, a nearly 3 -fold increase in the bR photovoltaic output. 


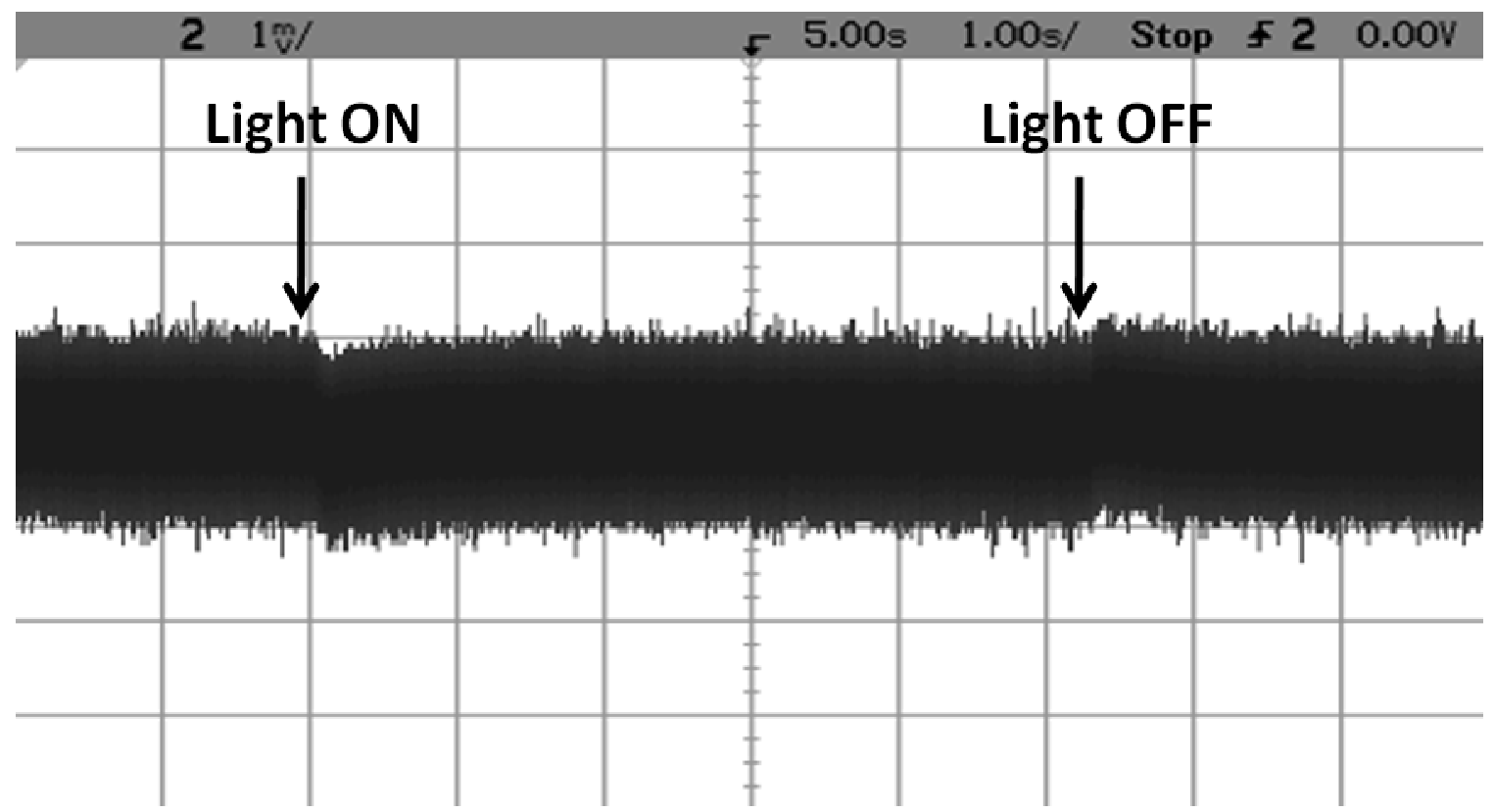

Figure 75. AC-coupled output of a 3 monolayer bR film (1 $1 \mathrm{mv} / \mathrm{div}, 1 \mathrm{sec} / \mathrm{div})$

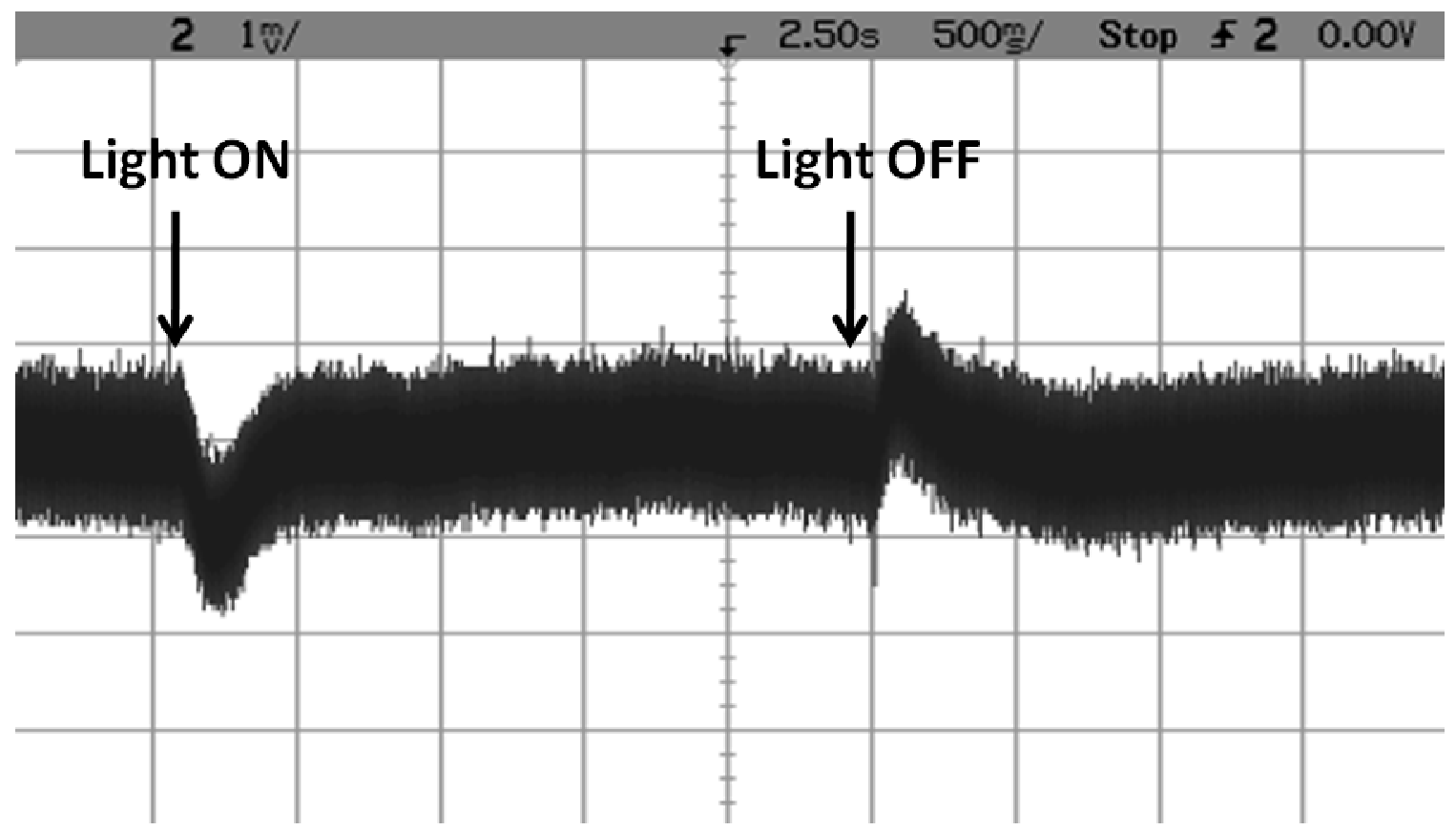

Figure 76. AC-coupled output of 3 monolayer bR film topped with 1 monolayer QD (1mV/div, 0.5sec/div). 


\section{Chapter 6. QD and bR FRET Coupling}

To modulate the photovoltaic output of this system, fluorescence resonance energy transfer (FRET) of the CdSe QD core and the bR retinal was investigated. A bR-QD FRET coupled system would allow for modulation of the QD photonic output based on proximity to the $\mathrm{bR}$ molecule. Ultimately this could provide a modulation point to achieve variable bR photovoltaic output upon toxin binding to the bR-QD coupled pair.

\subsection{THEORETICAL FRET CALCULATIONS}

In order to calculate the FRET efficiency between a donor and acceptor, the Forster radius must first be determined. The Forster radius is defined as the distance between the donor and acceptor where 50\% of the donor's energy is transferred via FRET. The Forster distance $\left(R_{o}\right)$ is defined as:

$$
R_{o}{ }^{6}=\left(8.8 \times 10^{23}\right)\left(\kappa^{2}\right)\left(\eta_{D}{ }^{4}\right)\left(\Phi_{D}\right)(J(\lambda))
$$

Where $\kappa$ is the dipole orientation factor, $\eta_{D}$ is the refractive index of the medium, $\Phi_{D}$ is the quantum yield of the donor, and $J$ is the normalized overlap integral between the donor and acceptor at each specific wavelength $(\lambda)$. Most of these variables have measurable values, but the $J$-integral must be calculated using the equation:

$$
J=\int f_{D}(\lambda) \varepsilon_{A}(\lambda) \lambda^{4} \partial \lambda
$$

Where $f_{D}$ is the peak normalized fluorescence spectrum of the donor, $\varepsilon_{A}$ is the molar absorption coefficient of the acceptor, and $\lambda$ is the wavelength. Once the Forster radius is determined, the FRET efficiency of the donor/acceptor pair at various separation distances can be determined. The FRET efficiency indicates the percentage of non- 
photonic emission from the donor, which is also the percentage of energy transferred to the acceptor through electron resonance. FRET efficiency $(E)$ can be calculated using the equation:

$$
E=\frac{R_{o}{ }^{6}}{R_{o}{ }^{6}+R^{6}}
$$

Where $R_{o}$ is the Forster radius for $50 \%$ resonance transfer and $R$ is the physical separation distance between the donor and acceptor molecules being studied. Using equation 6.2, a $J$-integral value of $6.12 \times 10^{-13} \mathrm{M}^{-1} \mathrm{~cm}^{3}$ was determined for bR with $565 \mathrm{~nm}$ emission QDs and $5.82 \times 10^{-13} \mathrm{M}^{-1} \mathrm{~cm}^{3}$ for bR with $600 \mathrm{~nm}$ emission QDs. These $J$-integral values along with the known variable values listed below were used to calculate the Forster radius using equation 6.1 .

$$
\begin{array}{lll}
\text { - } & \kappa=0.66 & \text { (dipole orientation factor) } \\
\text { - } & \Phi_{\mathrm{D}}=0.5 & \text { (quantum yield of } \mathrm{QD}) \\
\text { - } & \eta_{\mathrm{D}}=1.33 & \text { (refractive index of water) } \\
\text { - } & \varepsilon_{\mathrm{A}}=63,000 \mathrm{M}^{-1} \mathrm{~cm}^{-1} & \text { (bR extinction coefficient) }
\end{array}
$$

The Forster radius $\left(\mathrm{R}_{\mathrm{o}}\right)$ was theoretically calculated to be $7.62 \mathrm{~nm}$ for a $565 \mathrm{~nm} \mathrm{QD} / \mathrm{bR}$ system and $7.54 \mathrm{~nm}$ for a $600 \mathrm{~nm} \mathrm{QD} / \mathrm{bR}$ system. Therefore with a separation distance of 7.62nm between the $565 \mathrm{~nm}$ emission QD and a bR molecule, half of the QD energy will be transferred to the $\mathrm{bR}$ molecule non-photonically. The theoretical calculations also suggest that increasing the peak QD emission by $35 \mathrm{~nm}$ only decreases $R_{0}$ by $0.08 \mathrm{~nm}$, thus the use of QD emission precision at $570 \mathrm{~nm}$ is not critical.

After the Forster radius was determined, the FRET efficiency at varying QD/bR separation distances was determined. Using equation 3, the FRET efficiency for a $565 \mathrm{~nm}$ QD/bR system at varying separation distances $(\mathrm{R})$ was calculated and is shown in Figure 77. 


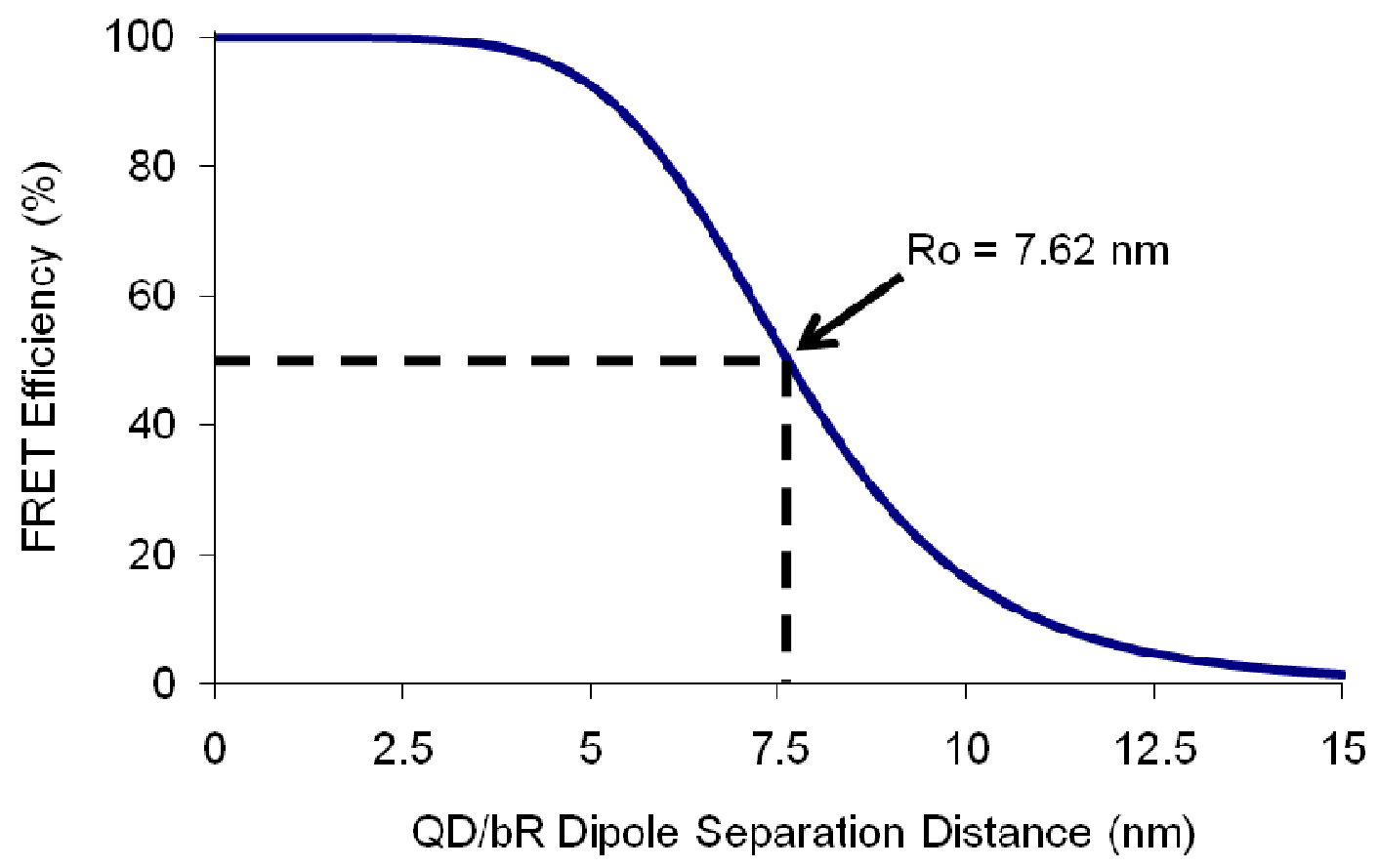

Figure 77. Theoretical FRET efficiency of a $565 \mathrm{~nm}$ QD-bR coupled system.

It can be noted from Figure 77 that the degree of FRET efficiency can vary greatly over a small change $(<1 \mathrm{~nm})$ in separation distance, particularly around the Forster radius $(\mathrm{E}=$ $50 \%$ ). For the bR-QD work a CdSe QD core and bR retinal separation distance of $7.62 \mathrm{~nm}$ will allow for the most sensitive FRET coupled pair. Implying that a small change in the bR-QD separation distance around the Forster radius, on the order of Angstroms, could provide sufficient alteration in QD emission to alter the bR photovoltaic response.

\subsection{AQUEOUS BR-QD FRET COUPLED SYSTEM}

Due to the large variation in FRET efficiency over a small change in separation distance, it will be important to maintain constant $b R / Q D$ proximity while performing the FRET 
study. In an aqueous suspension uniform bR-QD proximity can be achieved using protein and molecular-based linking schemes.

\subsubsection{Biotin-Streptavidin for bR-QD Linkage}

This separation distance was attained through the use of a biotin-streptavidin linkage. The small biotin molecule has an extremely high binding affinity for the streptavidin protein, to help ensure specific and tight binding at all locations. Streptavidin, a 53kDa protein, and biotin, also known as vitamin $\mathrm{H}_{\text {or }} \mathrm{B}_{7}$, are together widely regarded as the strongest non-covalent bond in nature, with a dissociation constant $\left(\mathrm{K}_{\mathrm{d}}\right)$ of $4 \times 10^{-14} \mathrm{M}$ [104]. The dissociation constant measures the concentration of the ligand at which half of the protein binding site is occupied. Thus with a $\mathrm{K}_{\mathrm{d}}$ of $4 \times 10^{-14} \mathrm{M}$, or $0.4 \mathrm{fM}$, the biotin-streptavidin system has a higher binding strength than the average antigenantibody system, which has a $\mathrm{K}_{\mathrm{d}} 10^{-7} \mathrm{M}$ to $10^{-11} \mathrm{M}$. Breaking this bond can only be achieved with harsh treatments or environmental temperatures in excess of $70^{\circ} \mathrm{C}$ [105]. With its robust nature and virtually unbreakable bond, the biotin-streptavidin binding scheme has been well studied in applications of nanoparticle linkers [106-110]. In these studies the biotin-streptavidin linkage was utilized to link biotinylated bR with streptavidin coated quantum dots, as shown in Figure 78. The estimated bR retinal/QD separation distance due to this bond is $\sim 8.5 \mathrm{~nm}$, assuming the streptavidin dimension of $5 \mathrm{~nm}$ [118], negligible biotin dimensions, QD shell thickness of 1.0nm, and retinal location in the center of $\mathrm{bR}, 2.0 \mathrm{~nm}$ from the biotin/streptavidin bond.

For this work, it was possible to biotinylate a single point on the bR molecule. Specifically this linkage occurs on the lysine 129 (K19) residue on the extracellular side of the protein when reacted at the proper $\mathrm{pH}$ [119]. It has also been shown that $\mathrm{bR}$ retains its functionality after biotin attachement [120] Thus, when streptavidin coated quantum dots were incorporated into the system, they attached to the available biotin molecules on the $\mathrm{bR}$ at a single point. 


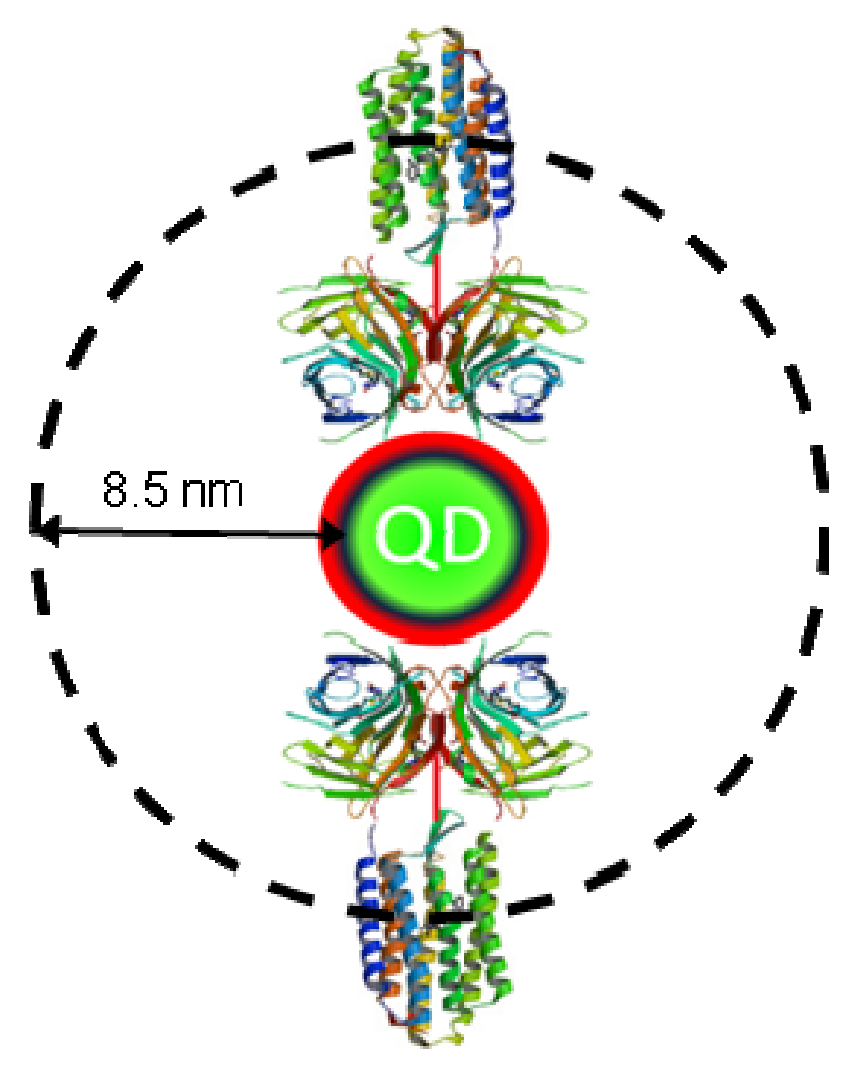

Figure 78. Schematic of bR-QD linkage via a biotin-streptavidin binding scheme.

The first step in this procedure was to biotinylate the $\mathrm{bR}$ molecule, which has been explored by various research groups [111-113]. To biotinylate the single bR lysine 129 residue it was critical to perform the reaction in the $\mathrm{pH}$ 8.3-8.5 range. Therefore the PM fragments were suspended in freshly made $0.1 \mathrm{M}$ sodium bicarbonate buffer; previously adjust to $\mathrm{pH} 8.4$ with $5 \mathrm{M} \mathrm{KOH}$. The PM was suspended to a concentration of $2.5 \mathrm{mg} / \mathrm{ml}$. The solution was then lightly sonicated to break up any large PM aggregrates and the $\mathrm{pH}$ was re-checked to ensure a proper $\mathrm{pH}$. A total volume of $115 \mu 1$ of the prepared PM suspension was removed and placed in a microcentrifuge tube on ice.

The biotin was prepared once the PM was ready for biotinylation. In a separate tube, on ice, $100 \mu \mathrm{l}$ of chilled sterile water was added. A total of $0.83 \mathrm{mg}$ of biotin (MW 556.58) was added to the tube and pipetted up and down to ensure the biotin was completely 
dissolved. This biotin concentration led to a 27:1 biotin:bR molar ratio in the final mixture. The tube containing the biotin solution was then vortexed and briefly microcentrifuged to collect droplets on the tube wall.

At this point the biotin was ready to be added to the PM solution. A total of $20 \mu 1$ of the biotin solution was pipetted into the prepared PM solution and mixed by pipetting up and down. The mixed solution was briefly microcentrifuged to remove droplets and then the reaction was allowed to run for 1 hour at room temperature. Following biotinylation the excess biotin was removed through a desalting column. The biotinylated bR could be used immediately or stored at $-80^{\circ} \mathrm{C}$ for future studies.

Utilizing a HABA/Avidin assay the amount of biotin attached to each bR molecule, on average, could be determined. Although the avidin proteins are most strongly bound to biotin, they also have marginal binding affinity for other materials. The HABA (4Hydroxyazobenzene-2carboxylic acid) dye is one such material. Since HABA absorbs strongly in the $500 \mathrm{~nm}$ wavelength region, its concentration in solution can be analyzed. In a HABA/avidin solution, when biotin is added it will compete for the avidin binding site and displace the HABA. The amount of displaced HABA was then analyzed based on the change in $\mathrm{A}_{500 \mathrm{~nm}}$ and was correlated to the amount of biotin added to the system. The blue markers and trend line in Figure 79 represent the change in HABA $\mathrm{A}_{500 \mathrm{~nm}}$ at varying concentrations of added biotin. Once the control baseline was determined, the biotinylated bR was added to a HABA/avidin solution and the change in HABA absorbance was quantitatively correlated to the amount of biotin present in the biotinylated $b R$ solution. The biotinylated $b R$ test samples are represented by the red markers in Figure 79. Based on the amount of $b R$ added, this can be further correlated to the amount of biotin bound to each bR molecule, as displayed in Table 2. 


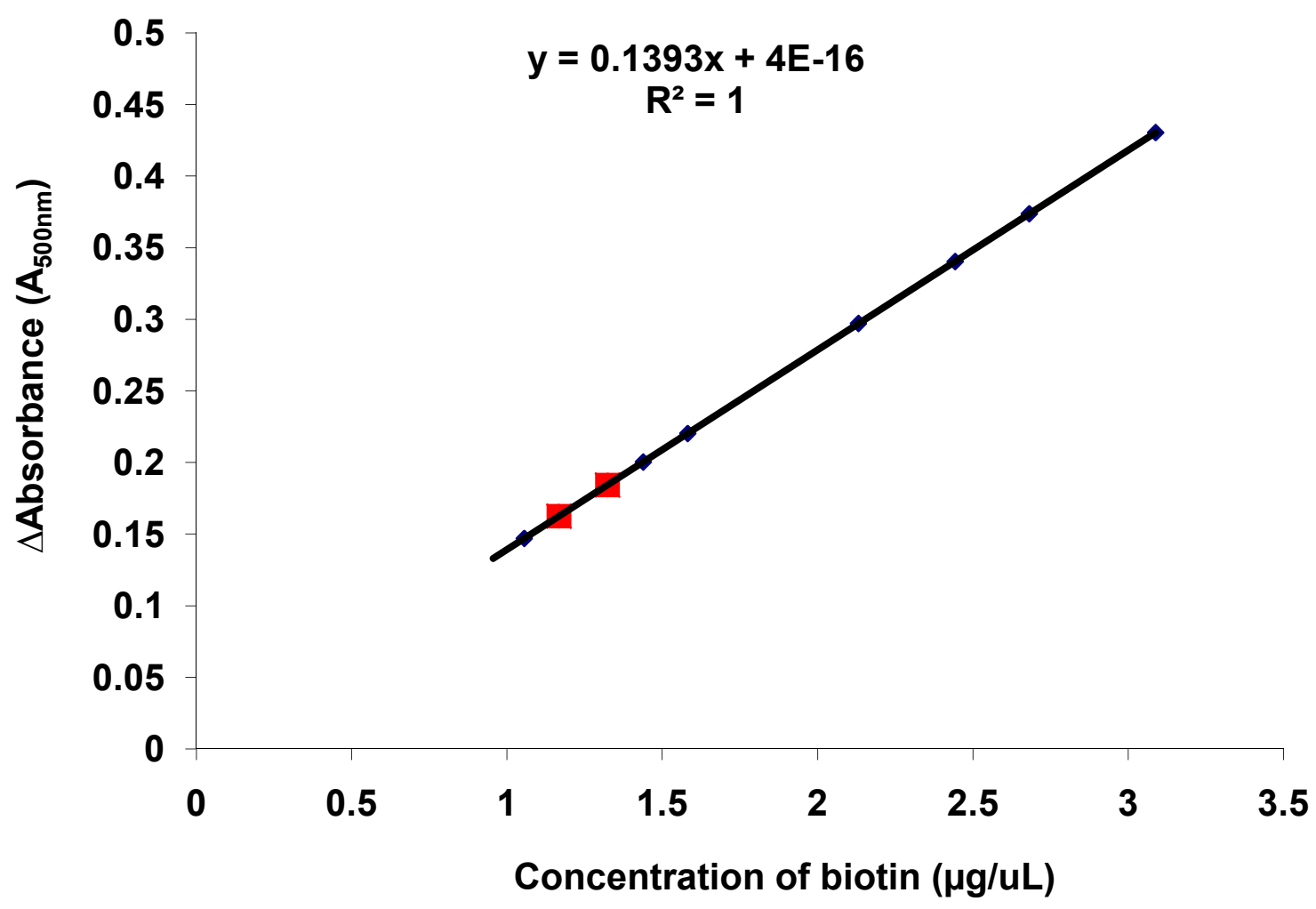

Figure 79. HABA/avidin assay for quantifying biotinylation of $b R$ molecules. Blue squares are control baseline and red squares are the biotinylated bR samples.

Table 2. Amount of biotins bound to bR based on HABA/avidin assay.

\begin{tabular}{|c|c|c|c|}
\hline $\begin{array}{c}\text { Biotinylated bR Conc. } \\
\text { (umole/ml) }\end{array}$ & $\Delta$ Abs. $\mathbf{5 0 0 n m}$ & $\begin{array}{c}\text { Biotin Conc. } \\
\text { (umole/ml) }\end{array}$ & $\begin{array}{c}\text { Biotin:bR } \\
\text { Molar ratio }\end{array}$ \\
\hline $1.15 \mathrm{E}-02$ & 0.1626 & $4.78 \mathrm{E}-03$ & 0.41 \\
\hline $1.35 \mathrm{E}-02$ & 0.1843 & $5.42 \mathrm{E}-03$ & 0.40 \\
\hline
\end{tabular}

Based on these results, there are 4 biotin molecules for every $10 \mathrm{bR}$ molecules, or roughly $50 \%$ of the bR molecules are biotinylated. This low biotin:bR ratio shows that the biotinylation procedure was likely successful at targeting the single lysine 129 residue on the bR molecule for biotin binding. The ability to target a single biotin group on the bR molecule will have applications for selective orientation of the bR molecule towards the streptavidin. 
Utilizing the separation distance achieved through the biotin-streptavidin linkage, the potential for FRET coupling between bR and QDs was tested in an aqueous environment. For this study, $1 \mu \mathrm{l}$ of $2.5 \mathrm{nmol} / \mathrm{mL}$ streptavidin coated quantum dots was suspended in 3 $\mathrm{mL}$ of deionized/distilled water. The fluorescence emission was initially measured using a SpexFluorolog spectroflourimeter. The biotinylated bR was added to the diluted QDs in small increments and the quenching of the QD fluorescence was analyzed. The incremental quenching of the streptavidin-coated QD emission upon addition of biotinylated bR is shown in Figure 80 and the reduction in QD peak emission is tracked versus bound bR amount in Figure 81. The computed FRET efficiencies at each bR:QD molar ratio is displayed in Table 3.

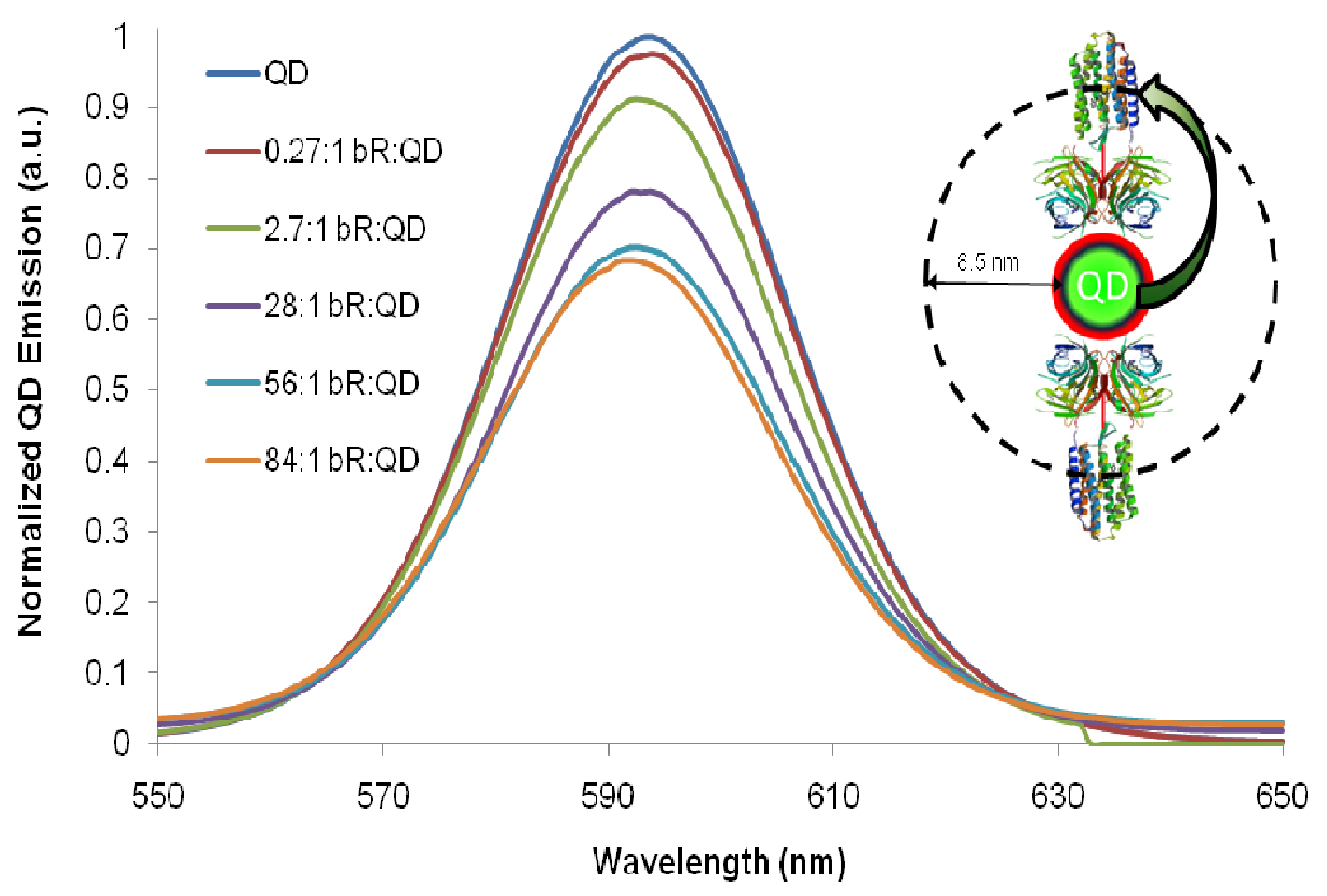

Figure 80. bR-QD FRET coupling via biotin-streptavidin linkage. 


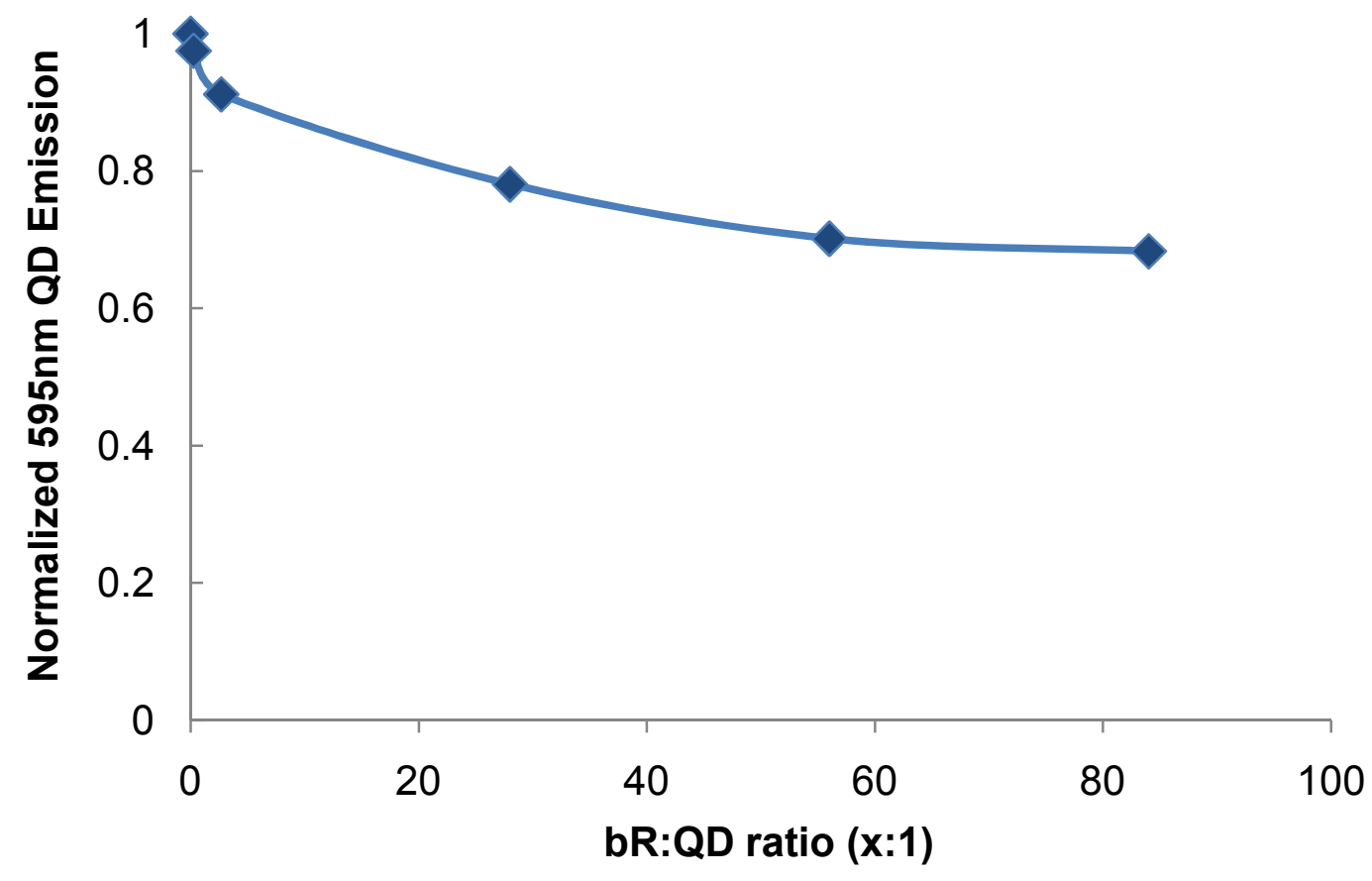

Figure 81. Tracking of normalized 595nm streptavidin QD emission as ratio of bound biotinylated bR is increased.

Table 3. FRET efficiency biotin-streptavidin linked bR-QD system at different molar ratios.

\begin{tabular}{|c|c|}
\hline bR:QD Molar Ratio & FRET Efficiency \\
\hline $0.27: 1$ & $2.47 \%$ \\
\hline $2.7: 1$ & $8.83 \%$ \\
\hline $28: 1$ & $21.93 \%$ \\
\hline $56: 1$ & $29.87 \%$ \\
\hline $84: 1$ & $31.71 \%$ \\
\hline
\end{tabular}

It can be seen that upon addition of biotinylated bR to the streptavidin coated quantum dots, the intensity of the quantum dot emission monotonically decreased. The quenching of QD emission was saturated when approximately $84 \mathrm{bR}$ molecules were present in the system for every 1 QD. Using the amount of QD quenching, it was determined that the 
FRET efficiency for this system, with a separation distance of $8.5 \mathrm{~nm}$, was $31.71 \%$. This implies that the QD was only emitting $68.29 \%$ of its normal photons, while the other $31.71 \%$ of its energy was being transferred to the bR molecule through resonance and photon absorption. This correlated closely with a theoretical FRET efficiency value of $32.80 \%$ for this separation distance, obtained from eq. (1)-(3).

From this study, a large number of bR molecules were necessary to reach the FRET maximum. It is expected that this large number of $\mathrm{bR}$ molecules was due to the relatively large size of the PM fragments. With PM having an average diameter of 470nm, it may have the tendency to fold upon itself. This will trap many of the bR molecules within the folds and make them inaccessible to the quantum dots. Therefore a majority of the bR molecules present in this system were probably not involved in the FRET process. The steric hinderance produced by the large PM fragments might have also hindered the approach of QDs to the biotinylated bR molecules.

\subsubsection{Zero-Length EDC for bR-QD Linkage}

To achieve extremely close bR-QD separation distances, a zero-space cross-linker called 1-ethyl-3-(3-dimethylaminopropyl) carbodiimide hydrochloride (EDC) was used. The EDC molecule is a water soluble carbodiimide that functions to form an amide linkage between a functional carboxyl and amine group [121]. It is composed of $\mathrm{C}_{8} \mathrm{H}_{17} \mathrm{~N}_{3} * \mathrm{HCl}$ and has a molecular weight of $191.7 \mathrm{~g} / \mathrm{mol}$. The chemical mechanisms leading to the amide linkage formation is shown in Figure 82.

In Figure 82, the ' 1 ' represents carboxyl functionalized QDs purchased from Invitrogen (Q21331MP) and '2' represents the bR molecule for this work. It is shown that the EDC cross-linker will form a stable amide bond between the QD carboxyl group and a nonspecific bR amine group. The amide bond is a 'zero-length' linker, allowing for the closest possible proximity between the bR molecule and QD nanoparticle. To prevent immediate hydrolization of the EDC intermediate, N-hydroxysulfosuccinimide (sulfo- 
NHS) was also implemented to convert the EDC-reacted carboxyl into a stable aminereactive sulfo-NHS ester [122]. This allows for a less-time dependant linkage reaction and ultimate creates more QD-bR amide linkages. The sulfo-NHS molecule has a molecular weight of $217.13 \mathrm{~g} / \mathrm{mol}$ and a chemical structure of $\mathrm{C}_{4} \mathrm{H}_{4} \mathrm{NNaO}_{6} \mathrm{~S}$. The end product of an EDC-linked bR-QD system is illustrated in Figure 83.

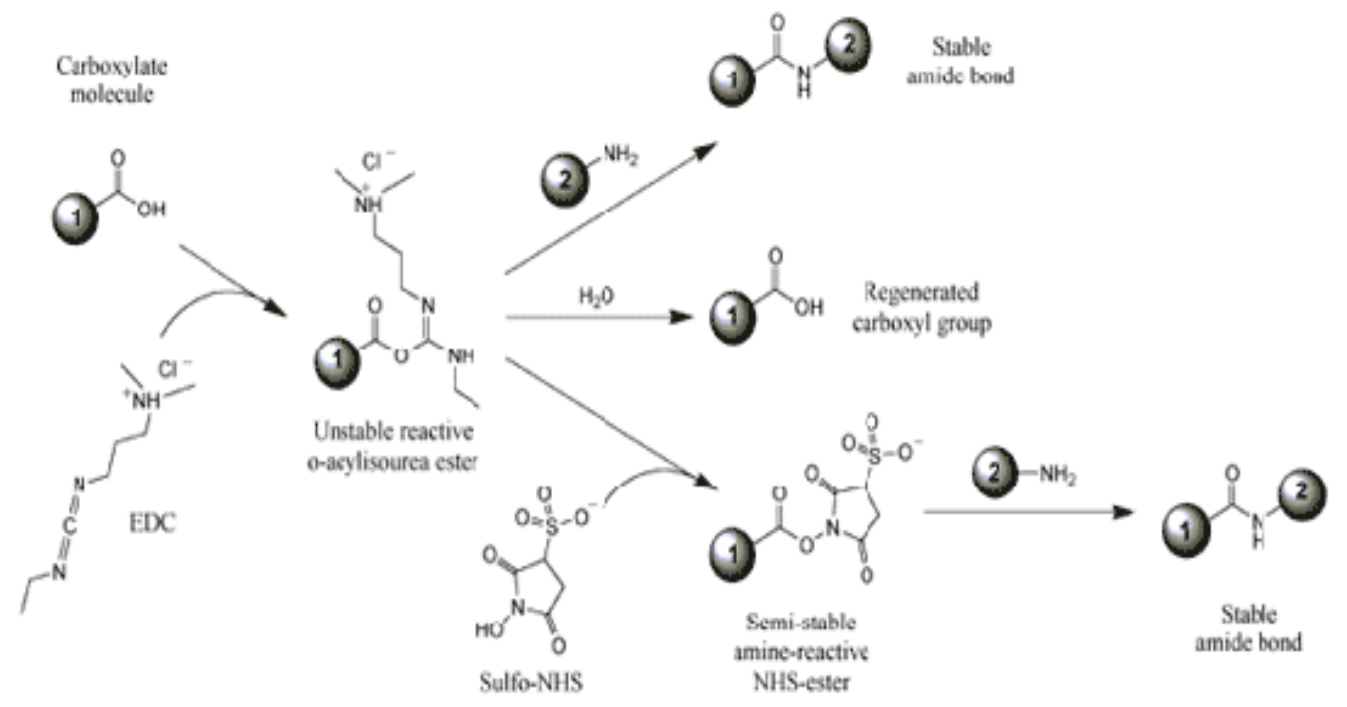

Figure 82. Schematic of EDC cross-linking mechanism (image courtesy of Pierce Technologies). Permission received from the Thermo Fisher Scientific and can be found in Appendix A.8.

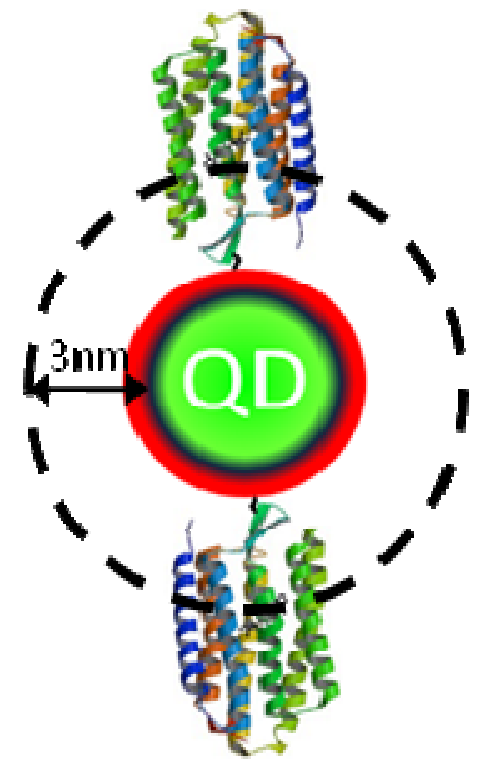

Figure 83. Schematic of bR-QD linkage via an EDC binding scheme. 
To efficiently create a QD-bR linkage utilizing an EDC cross-linker, the following procedure was followed to create a 40:1 bR:QD ratio. Initially, 25uL of Invitrogen ITK Carbolxyl QD (8 nmol/L stock) was added to $60 \mathrm{uL} 2-\mathrm{N}$-morpholino ethanesulfonic acid (MES) buffer solution. The MES buffer was prepared to $0.1 \mathrm{M} \mathrm{MES,} 0.5 \mathrm{M} \mathrm{NaCl}$ at $\mathrm{pH}$ 5.0. Care was taken to choose a buffer that would allow for low $\mathrm{pH}$ stability along with remaining un-reactive to the EDC molecule. In a separate vial $3 \mathrm{mg}$ sulfo-NHS was added to $0.5 \mathrm{~mL}$ MES buffer solution, followed by the addition of $1 \mathrm{mg}$ EDC. Once mixed, 25uL of the EDC/sulfo-NHS solution was added to carboxyl QDs to create aminereactive carboxyl groups. This step was reacted for 15 minutes and was ultimately quenched by the addition of $0.2 \mathrm{uL}$ of 2-mercaptoethnol to quench any un-reacted EDC. The non-reacted material was removed via a desalting spin column and the MES buffer was exchanged for a $50 \mathrm{mM}$ borate buffer, $\mathrm{pH} 8.3$.

The amine-reactive QDs were split into three equal volumes to perform three separate reactions. In this protocol three linkage reactions was performed with PM, bR monomers, and maltose binding protein, for reference. In each vial, $2.9 \mathrm{nmoles}$ of desired protein, also suspended in $50 \mathrm{mM}$ borate buffer $\mathrm{pH} 8.3$, was added to the QDs. This created an approximate 40:1 ratio of protein:QDs. The protein was mixed with the EDC functionalized QDs for 2 hours at room temperature to allow for maximal amide linkage formation. The un-reacted amine-reactive groups are quenched by the addition of $2 \mathrm{uL}$ hydroxylamine, which provided an excess of amines for binding. Finally, the reacted solution was filtered through a desalting spin column to remove the quenching agent. Care was taken to fully remove the hydroxylamine as previous work has shown this chemicals ability to cleave the bR retinal from the Schiff base, ultimately creating an apoprotein [123].

Utilizing the EDC linkage scheme to ensure QD-bR proximity, the energy coupling relationship between QDs and bR in the PM fragment form was analyzed. The QD quenching effects of the bR retinal in the PM fragment form is shown in Figure 84. The time progression of the QD quenching over the course of the EDC linkage step is displayed in Figure 85. 


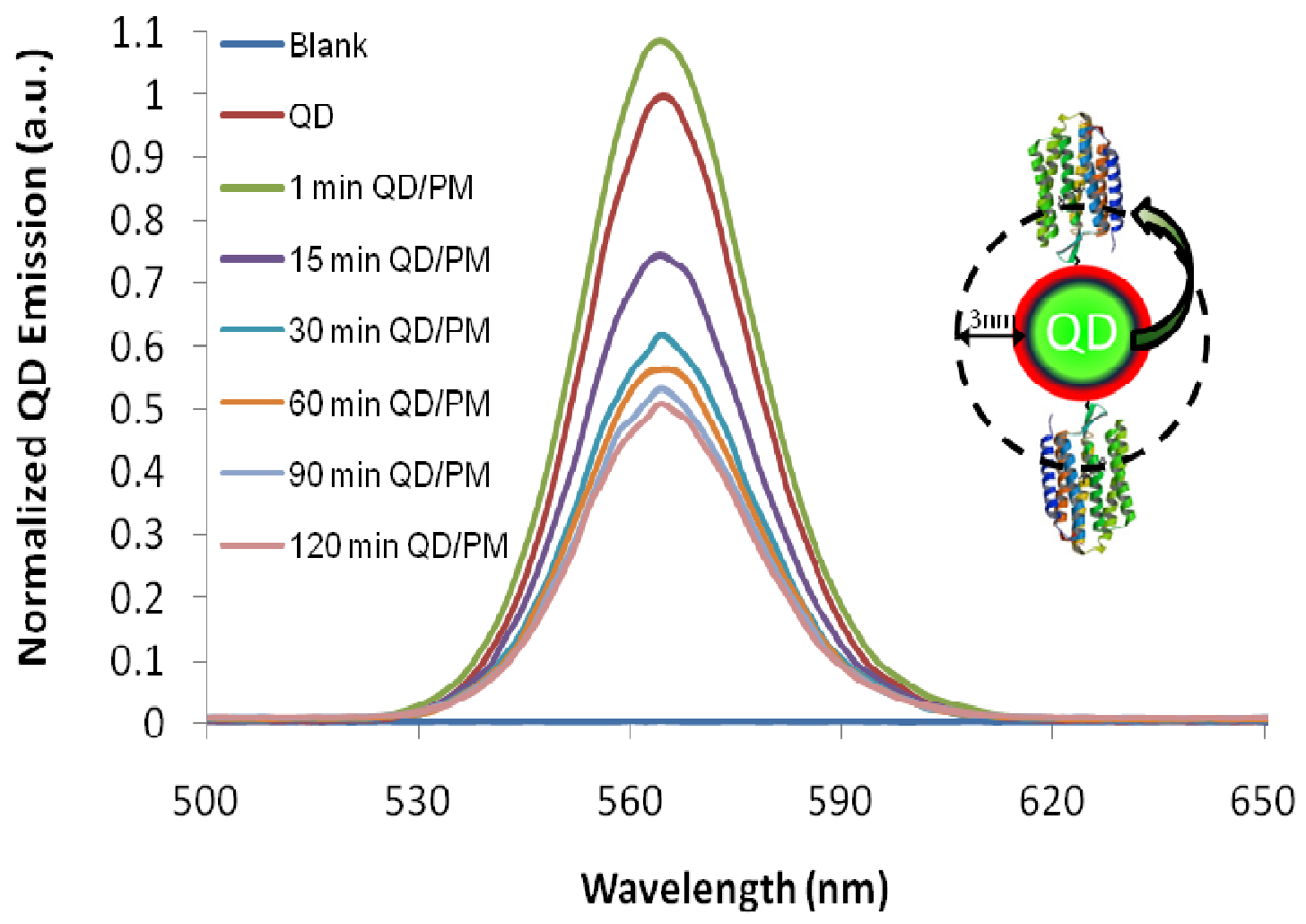

Figure 84. QD quenching effects of the bR retinal (bR in PM patch form) when linked to a CdSe/ZnS QD via EDC cross-linker.

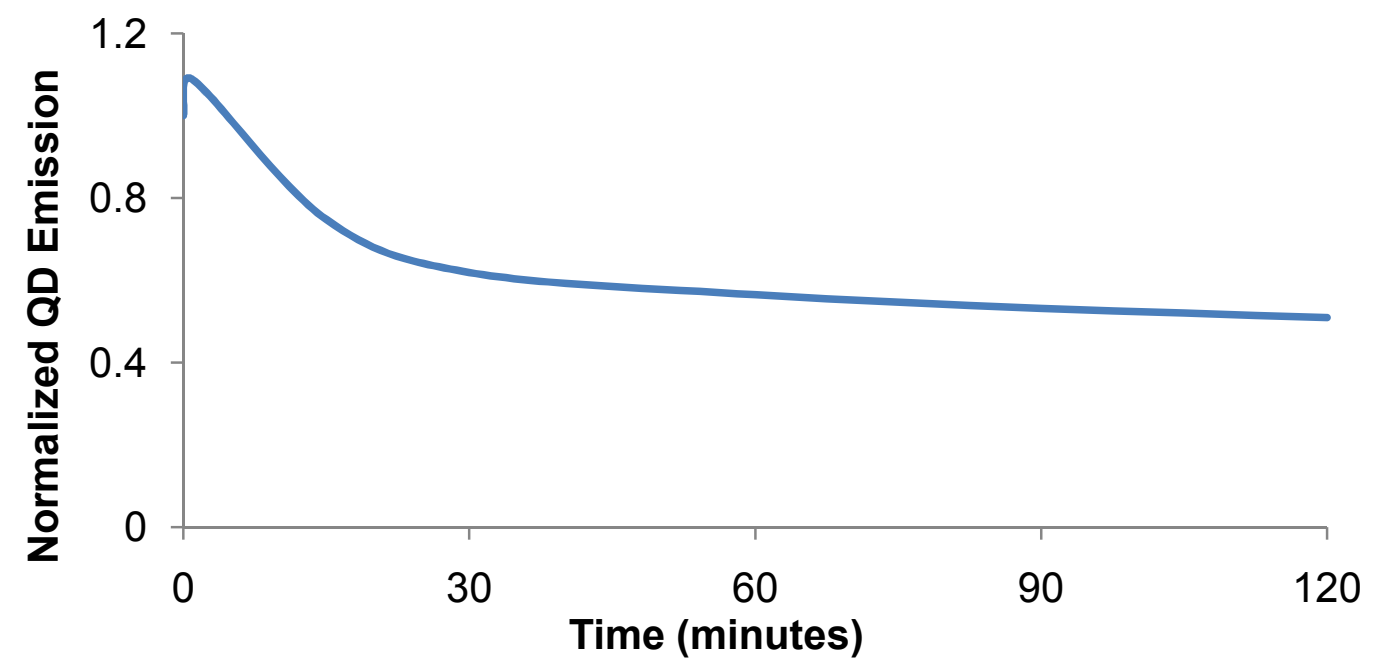

Figure 85. Time progression of QD quenching during EDC linkage with bR (PM patch form). 
The QD quenching phenomena shown in Figure 84 demonstrates energy transfer to the bR retinal from the excited CdSe QD. In the inset of Figure 84, a schematic of the 'zerolength' EDC linker is represented, showing the ability to bring the QD and bR molecule (40:1 bR:QD molar ratio) into a constant $\sim 3.5 \mathrm{~nm}$ separation distance. In addition Figure 85 suggests that the majority of the bR-QD amide linkages were formed within 30 minutes of EDC linkage initiation.

With the achieved bR-QD separation distance, a 49\% reduction in QD emission is observed when linked to bR in the purple membrane patch form, which is due to both photonic (photon absorption) and non-photonic energy transfer (FRET) events. The observed $49 \%$ of energy transfer is far less than the predicted value at the set separation distance, which is again due to bR being applied in its native PM patch form. The PM patch containing the $\mathrm{bR}$ and its associated lipids can be considered a macro-molecule, with a diameter of $\sim 470 \mathrm{~nm}$ that will fold and conform to its lowest energy state in the aqueous solution. The folding of this macro-molecule will result in many $\mathrm{bR}$ molecules being inaccessible to the QDs, ultimately inhibiting the number of bR molecules a single QD can couple with, thus reducing FRET efficiency.

\subsection{AQUEOUS BR-QD FRET WITH BR MONOMERS}

To reduce the fragment size of the PM patches, the use of a detergent to solubilize bR monomers was used. Specifically, the detergent octyl- $\beta$-D-glucoside (OG) was used. The addition of OG above its critical micelle concentration (CMC) of $25 \mathrm{mM}$ will allow the OG to penetrate and remove the PM lipid layer and form hydrophilic micelles with bR monomers [38]. Over time, on the order of a day or more, the presence of OG will denature $b R$. 


\section{$\underline{\text { 6.3.1 bR Monomer Preparation }}$}

The first step was to optimize the concentration of OG added to the PM solution and the amount of mixture time to provide the highest degree of protein solubilization while minimizing bR denaturation. In Figure 86, the degree of bR solubilization over the course of 24 hours, for several different OG concentrations, is shown. Simultaneous measurements to determine the extent of bR denaturation throughout this study are shown. The optimal $280 \mathrm{~nm}-570 \mathrm{~nm}$ absorption peak ratio for bR is 2 , any value higher than 2 implies that some of the bR molecules have denatured and are no longer funtional.

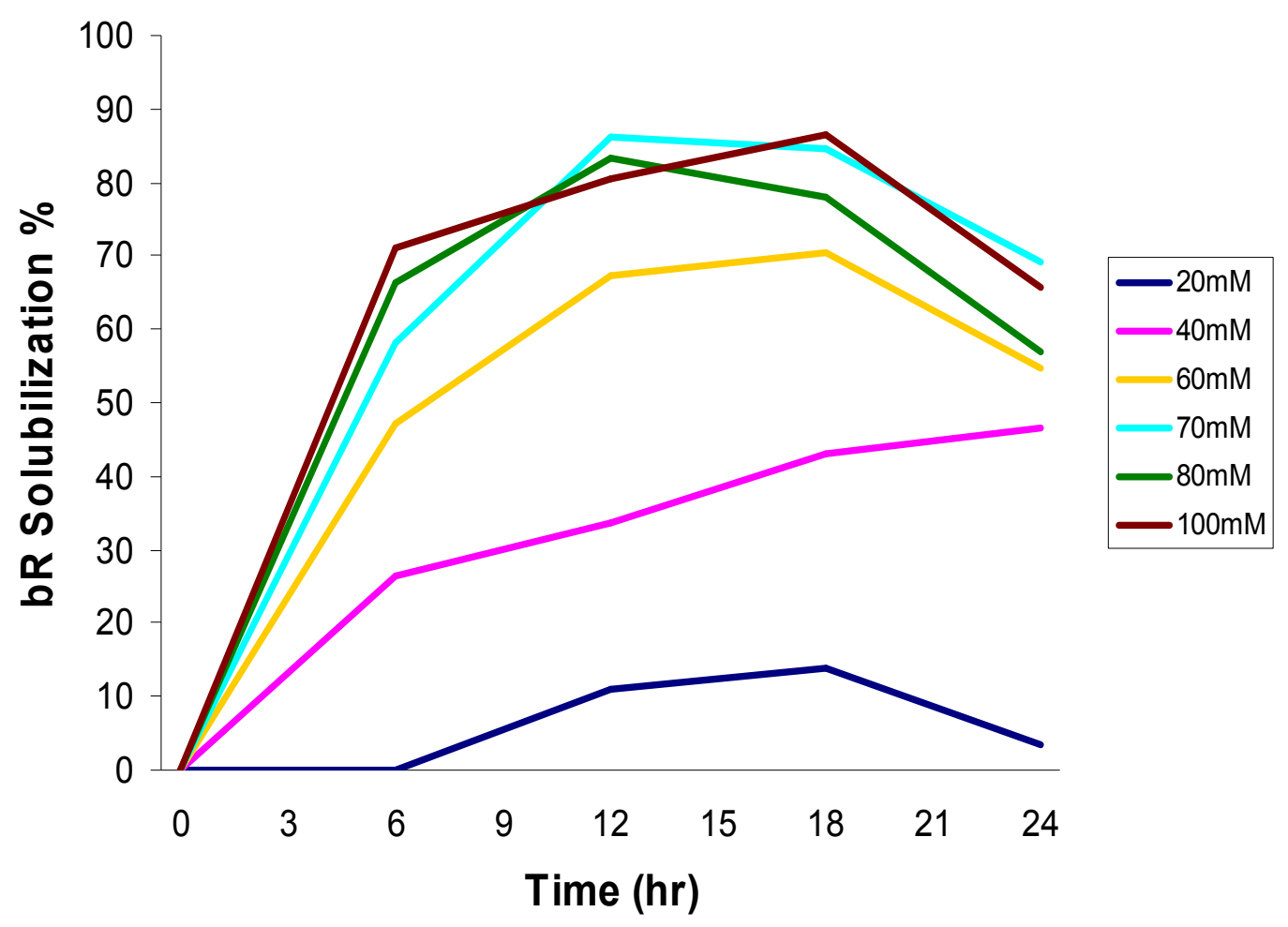

Figure 86. bR solubilzation vs. OG concentration over 24 hour duration. 


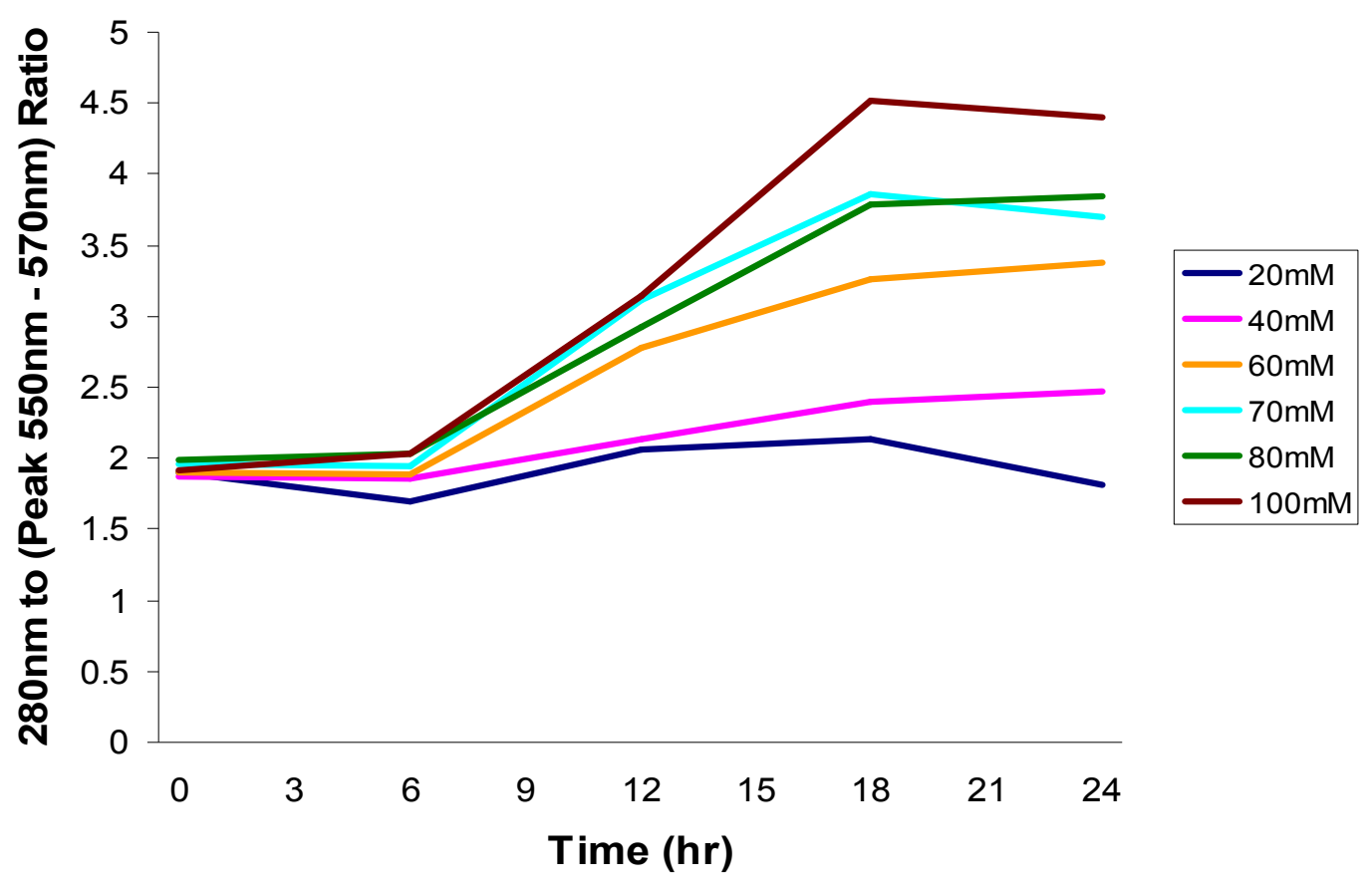

Figure 87. Degree of bR denaturation vs OG concentration over 24 hour duration

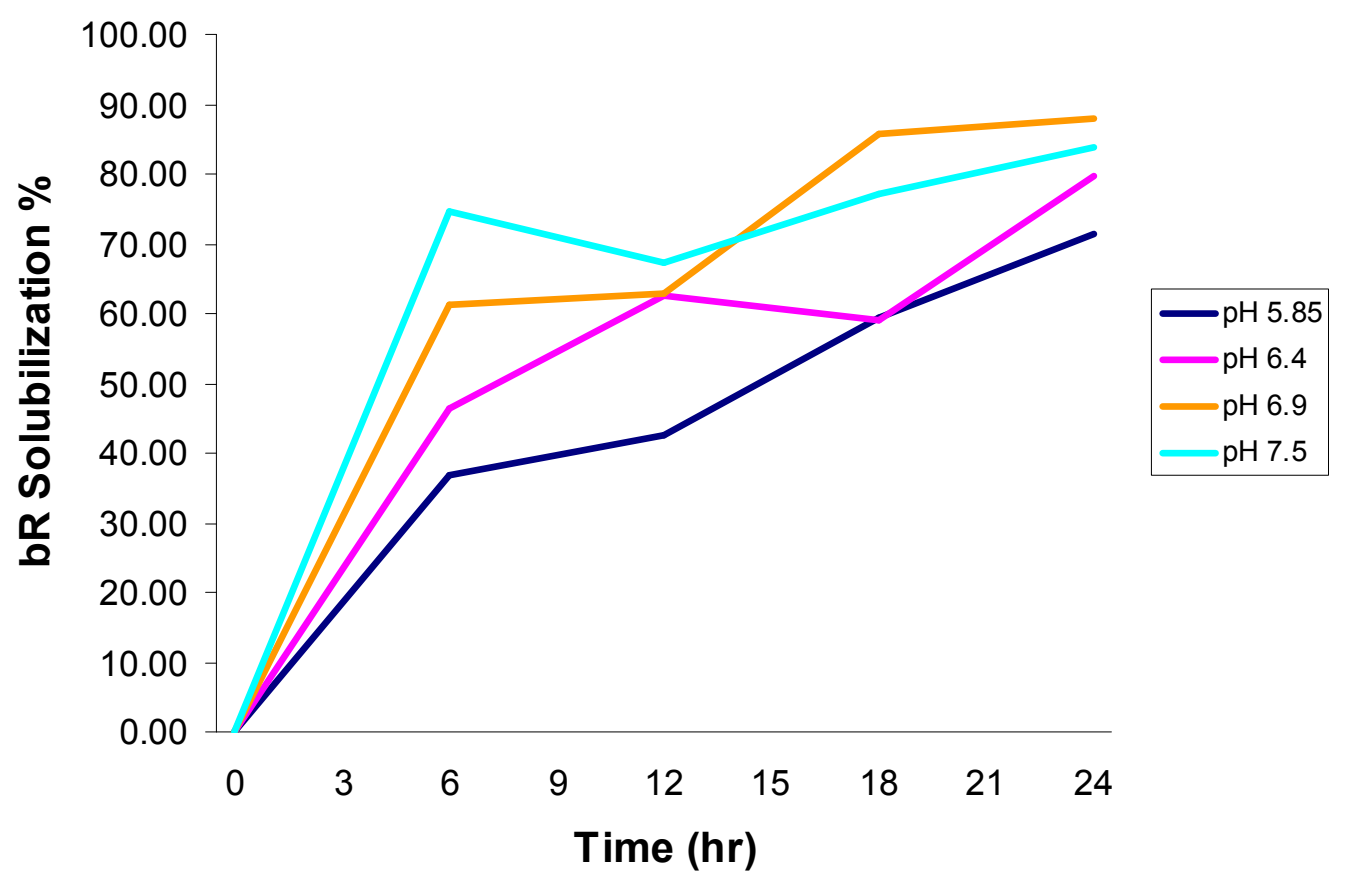

Figure 88. bR solubilization \% vs. 70mM OG pH over 24 hour duration 


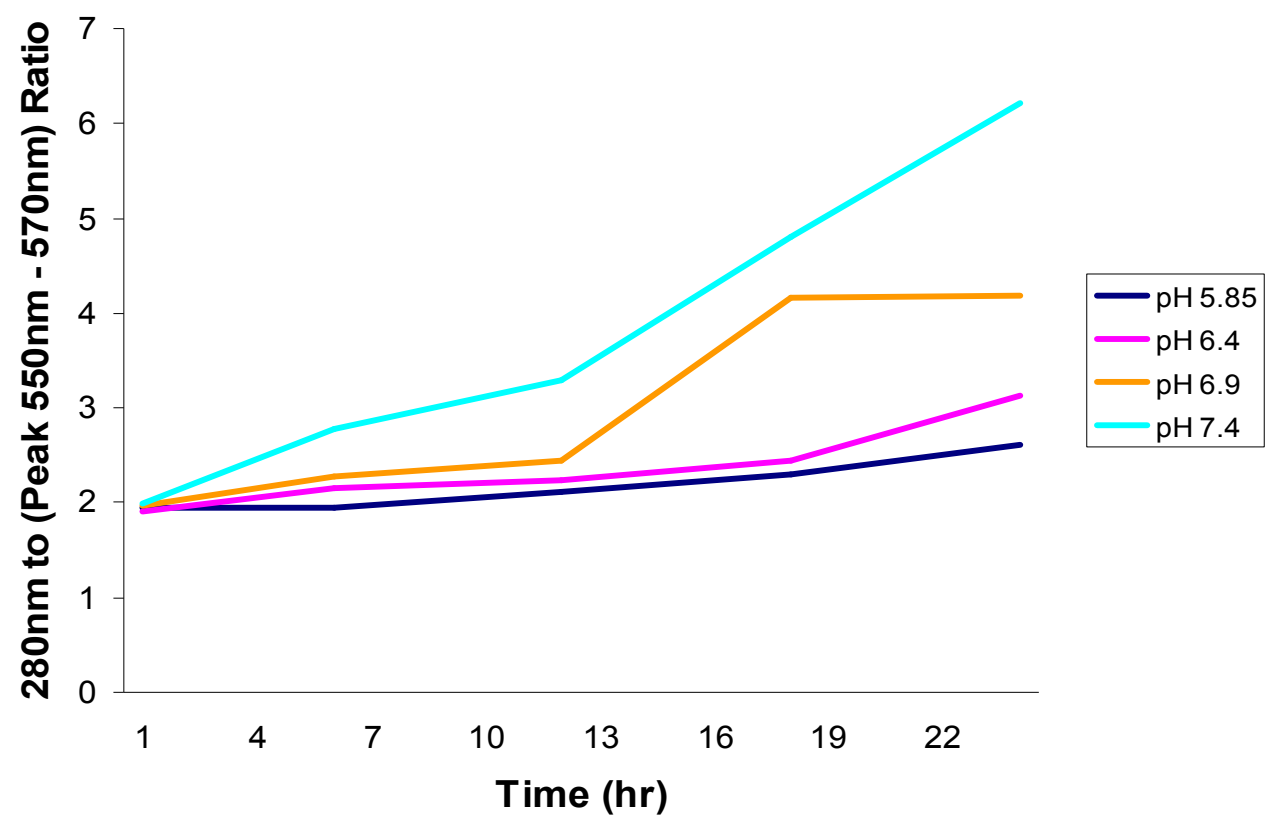

Figure 89. Degree of bR denaturation vs. 70mM OG pH over 24 hour duration

Upon analysis of the results shown in Figure 87, it appears that the optimal OG concentration ranged between $60 \mathrm{mM}$ and $80 \mathrm{mM}$. These values gave the highest degree of bR solubilization with relatively low bR denaturation. The results also show that the optimal length of time to solubilize the PM ranges between 6 and 12 hours. Using an OG concentration of $70 \mathrm{mM}$, the $\mathrm{pH}$ of the OG solution was studied. The results for bR solubilzation and denaturation with $70 \mathrm{mM}$ OG over a range of $\mathrm{pH}$ values is shown in Figure 88 and Figure 89, respectively. From the studies with $70 \mathrm{mM} \mathrm{OG}$, it was found that the optimal $\mathrm{pH}$ was 6.9 to relatively maximize solubilization with minimal bR denaturation.

\subsubsection{Biotin-Streptavidin for bR Monomer-QD Linkage}

For the QD FRET studies, 70mM OG solution of $\mathrm{pH} 6.9$ was added to $1.5 \mathrm{mg}$ of PM for approximately 12 hours. The solubilized bR was then non-preferentially biotinylated at a ratio of 6 biotin molecules per bR molecule. The excess biotin was removed by passing 
the solution through a sepharose $2 \mathrm{~B}$ column that was equilibrated with $70 \mathrm{mM}$ OG. The biotinylated/solublized bR monomer was combined with $2.5 \mathrm{nmol} / \mathrm{mL}$ streptavidin-coated $600 \mathrm{~nm}$ emission Evifluor quantum dots at varying volumes to create the desired bR:QD ratio. The degree of FRET quenching resulting from the different ratios of $b R$ monomer:QD is displayed in Figure 90. These results were normalized to the bR control (QD only emission) for ease of comparison. The calculated FRET quenching values are shown in Table 4.

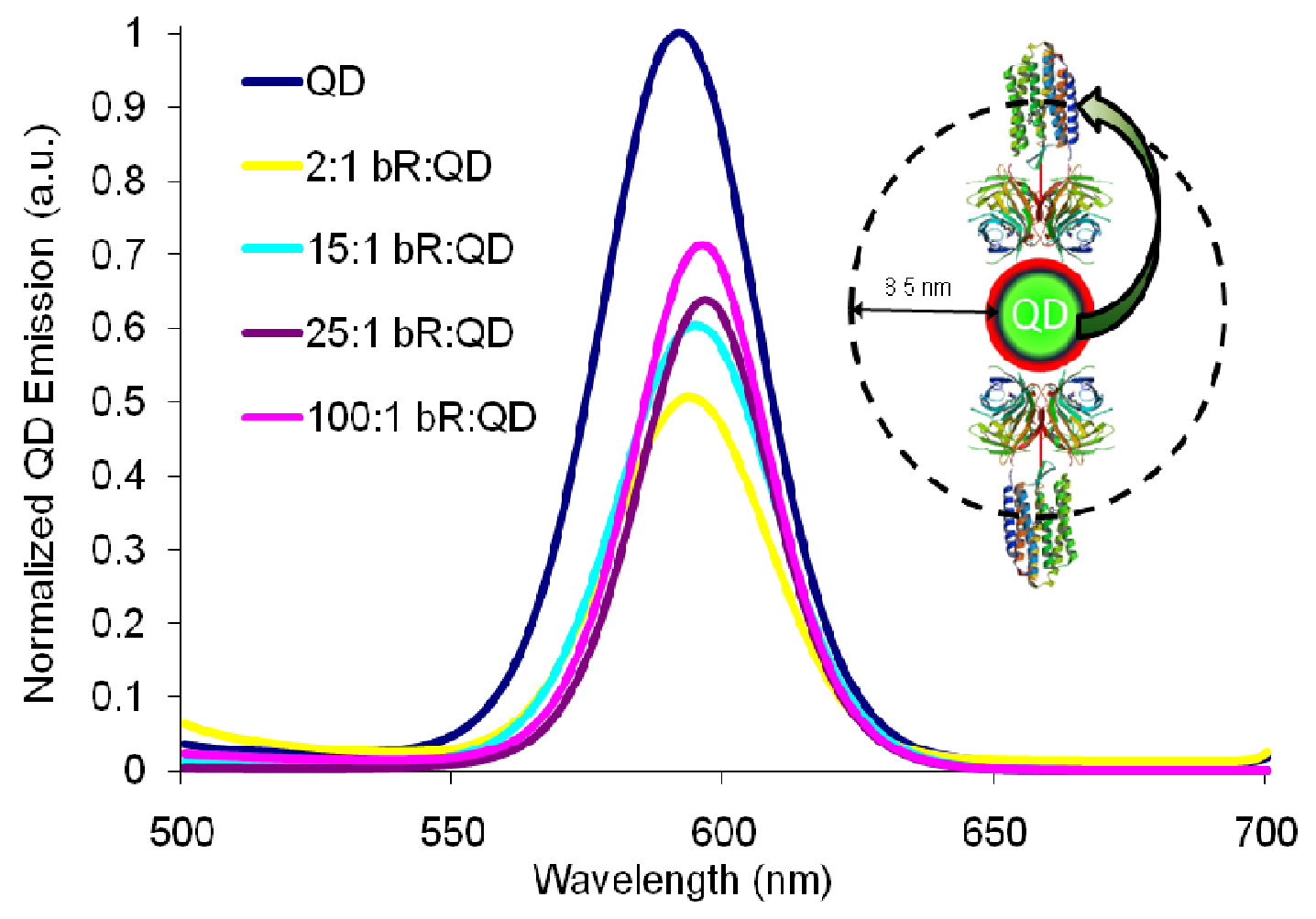

Figure 90. FRET quenching of various QD:bR monomer ratios, linked via biotin-streptavidin. 


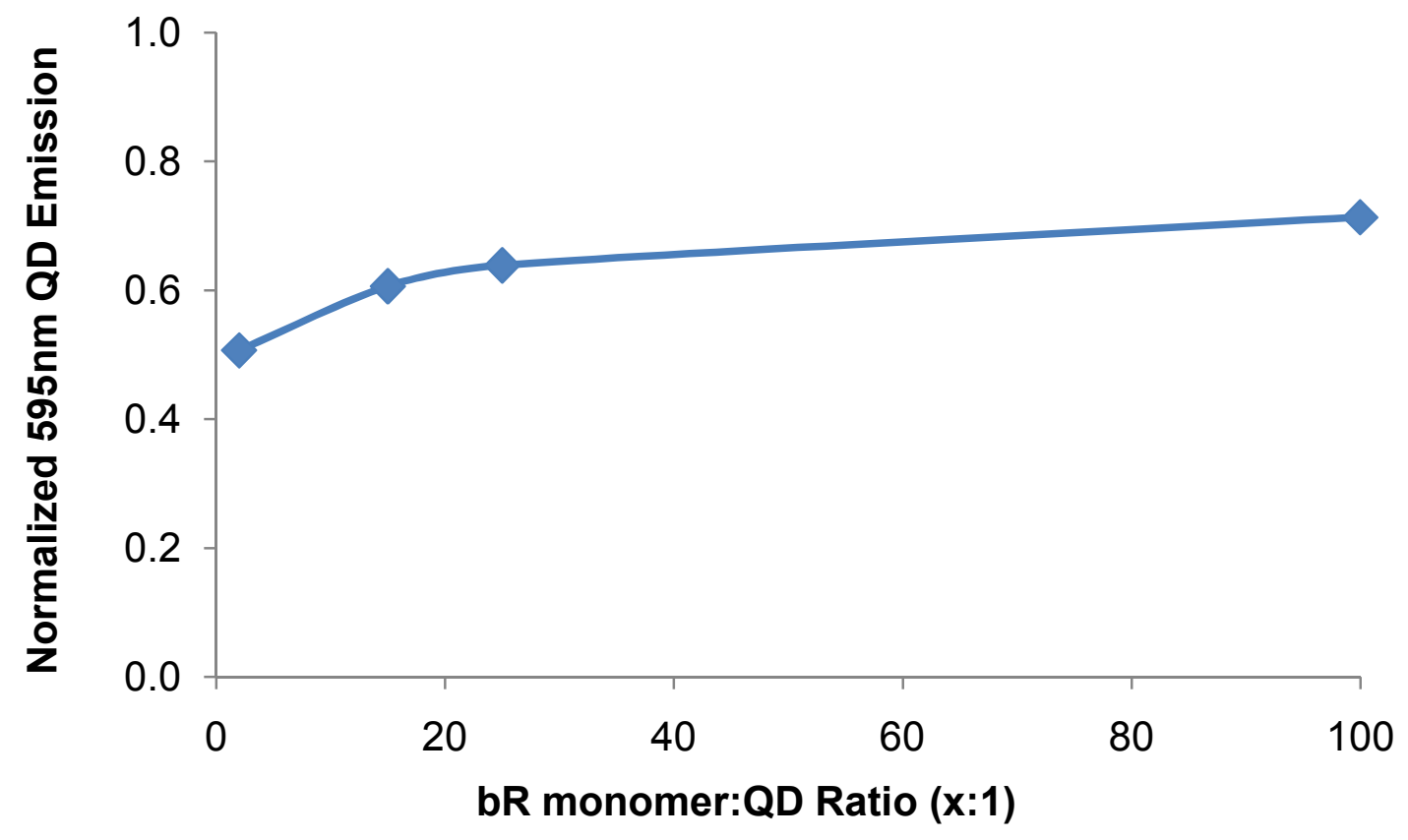

Figure 91. Tracking of normalized 595nm streptavidin QD emission as ratio of bound biotinylated bR is increased.

Table 4. FRET efficiency for varying QD:bR monomer ratios

\begin{tabular}{|c|c|}
\hline bR:QD Molar Ratio & FRET Efficiency \\
\hline $2: 1$ & $49.31 \%$ \\
\hline $15: 1$ & $39.36 \%$ \\
\hline $25: 1$ & $36.10 \%$ \\
\hline $100: 1$ & $28.64 \%$ \\
\hline
\end{tabular}

These results imply that lowering the bR:QD ratio increases the degree of FRET coupling between the bR molecule and QD. These phenomena could have been potentially due to a closer $\mathrm{bR} / \mathrm{QD}$ separation distance with decreased competition for the streptavadin sites. As shown in Figure 92, the results QD quenching characteristics of bR in its monomeric and PM fragment forms vary. 


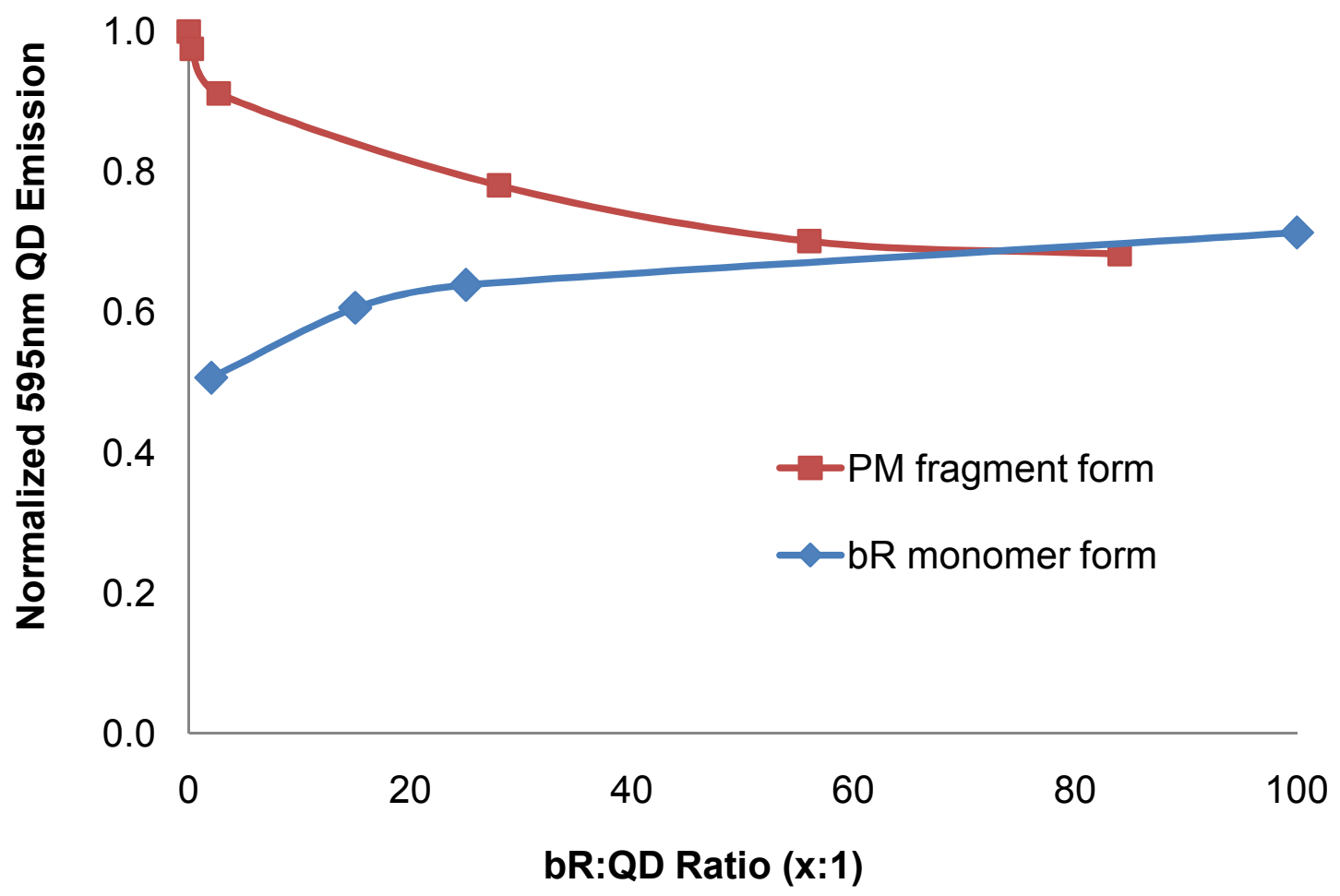

Figure 92. Comparison of QD quenching phenomena when linked via a biotin-streptavidin linkage to bR in both the PM fragment (red square) and bR monomer (blue diamond) forms.

At lower bR:QD ratios, bR in its monomeric form has a substantially higher FRET coupling efficiency than when bR is in its PM fragment form. As previously mentioned this is likely due to the steric hindrances of the larger PM fragment, ultimately hindering the accessibility of the present bR molecules. It is also evident from Figure 92 that the behavior of QD quenching versus bR:QD ratio varies between the monomeric and PM fragment forms. As the $\mathrm{bR}$ in PM fragment form induces an increasing degree of QD quenching in conjunction with elevated bR levels, elevated bR monomer levels actually leads to a decrease in FRET coupling efficiency. Possible explanation for these phenomena could be that increased competition of $b R$ monomers for the streptavidin at high ratios would alter the bR-QD separation distance or that the high concentrations actually induce a relipidation of the bR monomers back into the PM fragment form. 


\subsubsection{Zero-Length EDC for bR Monomer-QD Linkage}

Likewise with the aforementioned biotin-streptavidin linkage study, the bR monomers were prepared by mixing $1.5 \mathrm{mg}$ of $\mathrm{PM}$ in $70 \mathrm{mM}$ OG solution of $\mathrm{pH} 6.9$ for approximately 12 hours. The QDs were modified and linked to the bR monomers at a 40:1 ratio using the EDC linkage protocol previously described.

Utilizing the EDC linkage scheme to ensure QD-bR proximity, the energy coupling relationship between QDs and bR monomers was analyzed. The QD quenching effects of the bR retinal is shown in Figure 93. The time progression of the QD quenching over the course of the EDC linkage step is displayed in Figure 94.

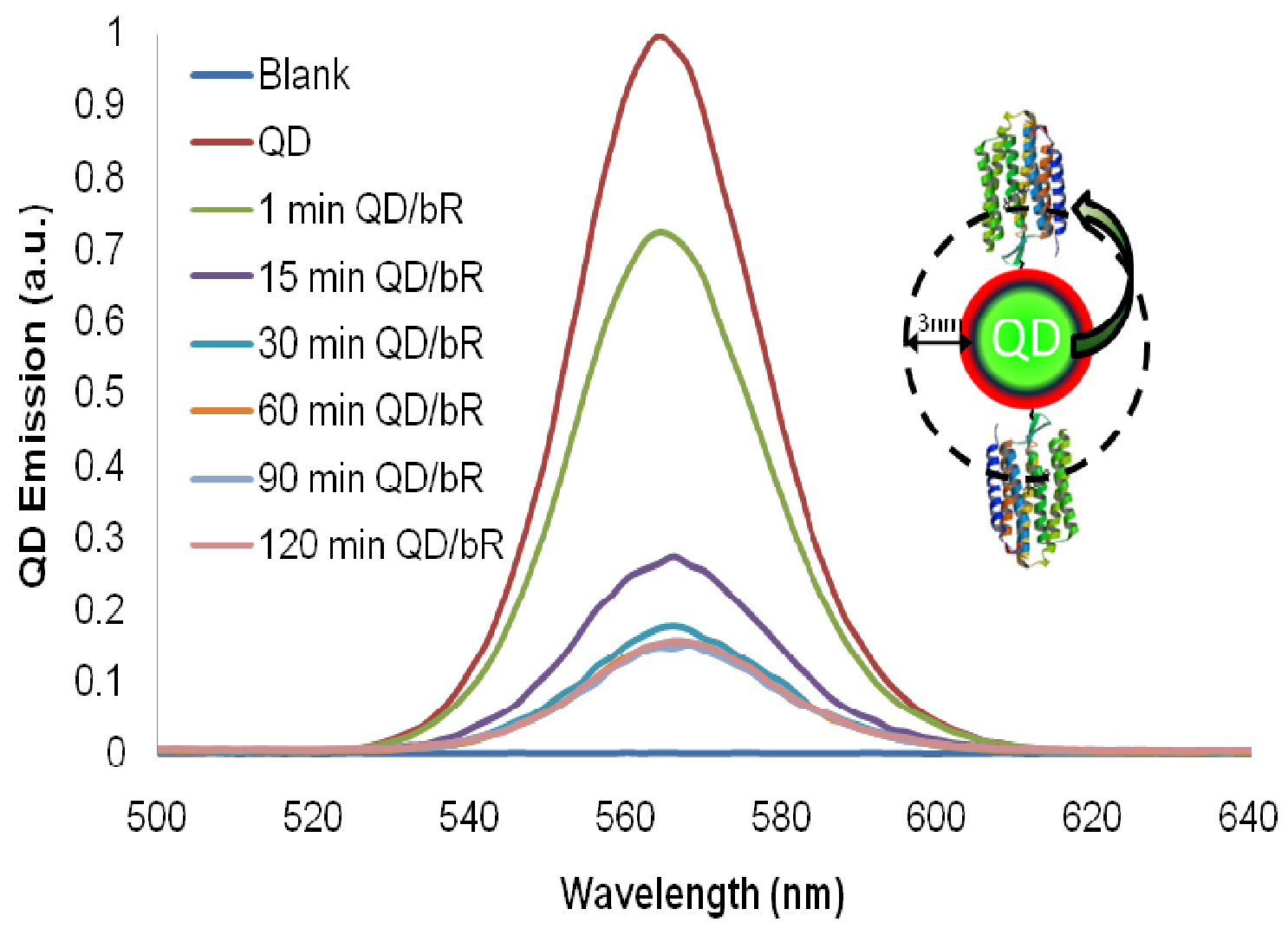

Figure 93. Progression of QD quenching during EDC linkage to bR monomers. Maximum FRET Efficiency of a coupled QD/bR monomer system is $88.2 \%$. 


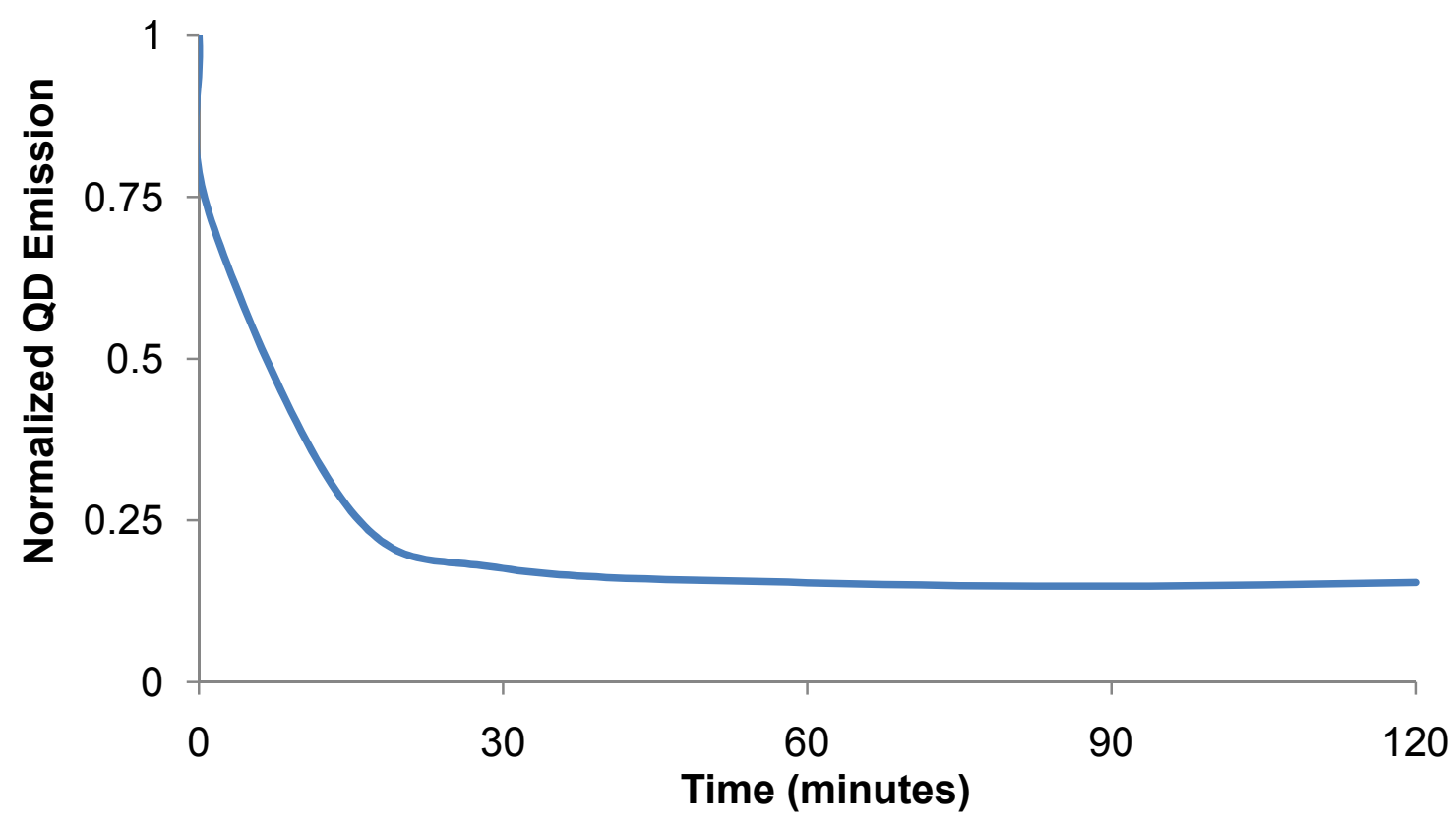

Figure 94. Time progression of QD quenching via EDC linkage reaction with bR monomers.

The QD quenching phenomena shown in Figure 93 demonstrates energy transfer to the bR retinal from the excited CdSe QD. In the inset of Figure 93, a schematic of the 'zerolength' EDC linker is represented, showing the ability to bring the QD and bR molecule (40:1 bR:QD molar ratio) into a constant $\sim 3.5 \mathrm{~nm}$ separation distance. In addition Figure 94 suggests that the majority of the bR-QD amide linkages are formed within 15-20 minutes of EDC linkage initiation.

With the achieved bR-QD separation distance a $88 \%$ reduction in QD emission was observed when linked to the bR monomer, which was due to both photonic (photon absorption) and non-photonic energy transfer (FRET) events. The observed $88 \%$ of energy transfer was close the predicted value at the set separation distance. Theoretical calculations on a bR-QD energy coupled system at this separation distance predicts near complete energy transfer, which would be difficult to obtain in experimental conditions due to the requirement of complete binding of every molecule. 
A comparison of the two different forms of bR when EDC linked to QDs is shown in Figure 95. This demonstrates the draw-backs of using large PM patches and that it's associated steric hindrance can be overcome with bR monomers. When compared to the EDC linkage of QDs to bR in the PM patch form, a 39\% increase in QD quenching is observed with bR monomers at the same molar ratio. In its monomeric form more of the bR molecules can access and bind to the functionalized QDs and thus put a greater quantity of bR retinals in FRET coupling proximity to the QDs.

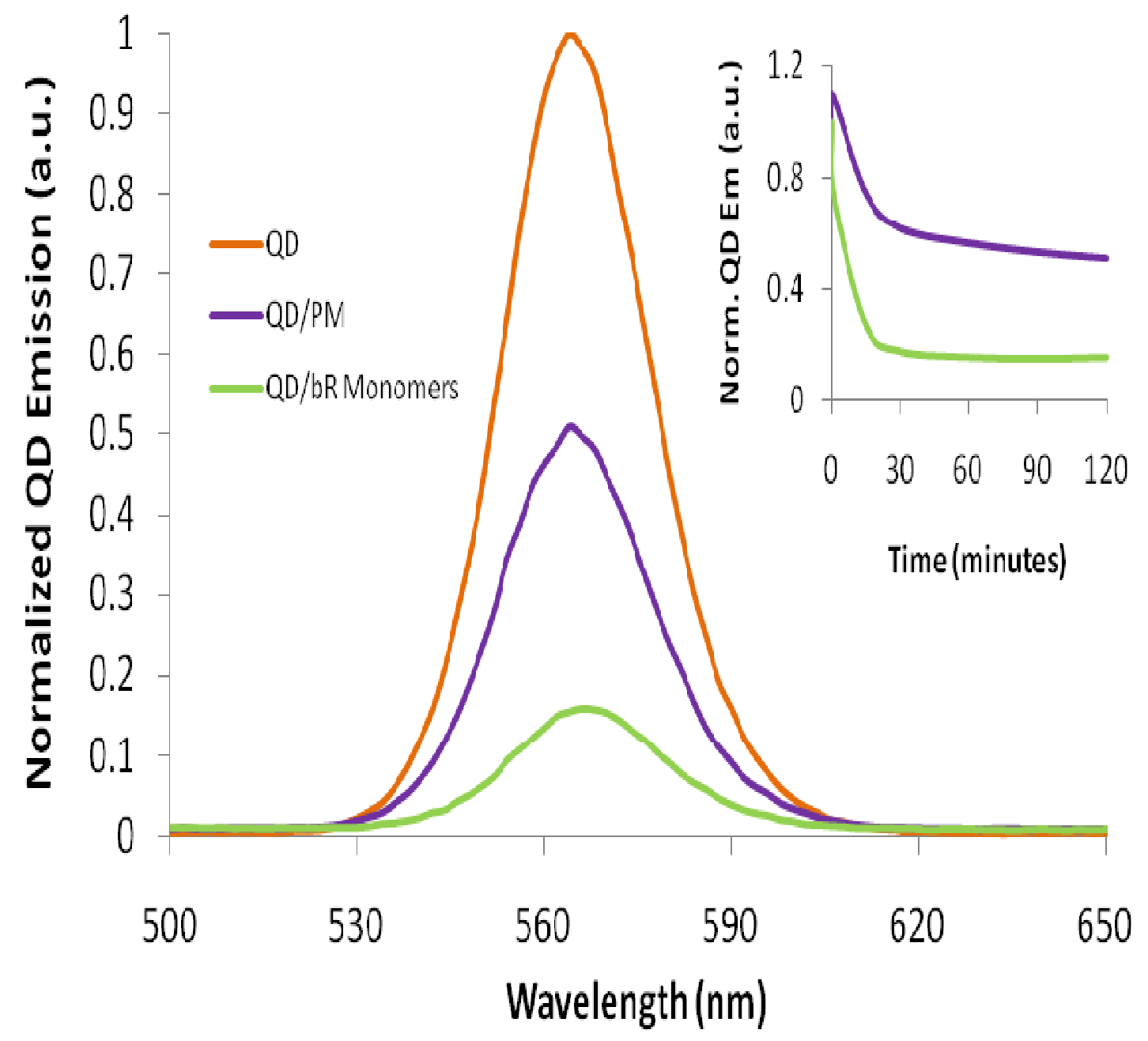

Figure 95. Comparison of QD quenching effects from EDC linked PM fragments and bR monomers. Maximum FRET Efficiency of a coupled QD/PM fragment system is $49.1 \%$ while a QD/bR monomer system yield 88.2\% FRET efficiency. Inset displays FRET coupling vs. EDC linkage reaction time. 
The inset of Figure 95 compares the degree of QD quenching achieved during the progression of EDC binding for $\mathrm{bR}$ monomers and $\mathrm{bR}$ in the PM fragment form. This comparison reveals several differences between utilizing bR monomers and PM fragments in a QD coupled system. First it can be noted that the bR monomers became linked and began quenching the QD emission much quicker than the PM fragments. Within 1 minute of EDC linkage initiation, the bR monomers FRET couple with the QDs at an efficiency of $27.5 \%$, compared to a slight increase in QD emission when the PM fragment linkage had been initiated for 1 minute. An increase in QD emission has been previously witnessed when in close proximity to a biomolecule with which it is not FRET coupling [124]. Additionally a maximal FRET coupling relationship was achieved within 15-20 minutes with bR monomers, compared to approximate maximum occurring after 30 minutes with the PM fragments. After this time point the FRET coupling relationship between EDC linked QDs and bR monomers remained fairly constant, whereas a gradual increase in the EDC linked QD-PM fragment system was seen. The gradual increase in coupling efficiency with PM fragments was due to the steric hindrance and accessibility to $b R$ in this macromolecule. Over extended time, some of the relatively inaccessible bR molecules can be linked to the QD in enhance the FRET coupling relationship.

To further clarify the importance of proximity between the QD core and $\mathrm{bR}$ retinal, the coupling efficiency of the biotin-streptavidin and EDC linked systems are shown in Figure 96. It is noted that the EDC linkage was performed with $565 \mathrm{~nm}$ emission carboxyl coated CdSe/ZnS QDs, while the biotin-streptavidin linkages were performed with $595 \mathrm{~nm}$ emission streptavidin coated CdSe/ZnS QDs. Minimal differences were seen in bR-QD FRET coupling efficiencies for these two different QDs, as shown in Table 5. 


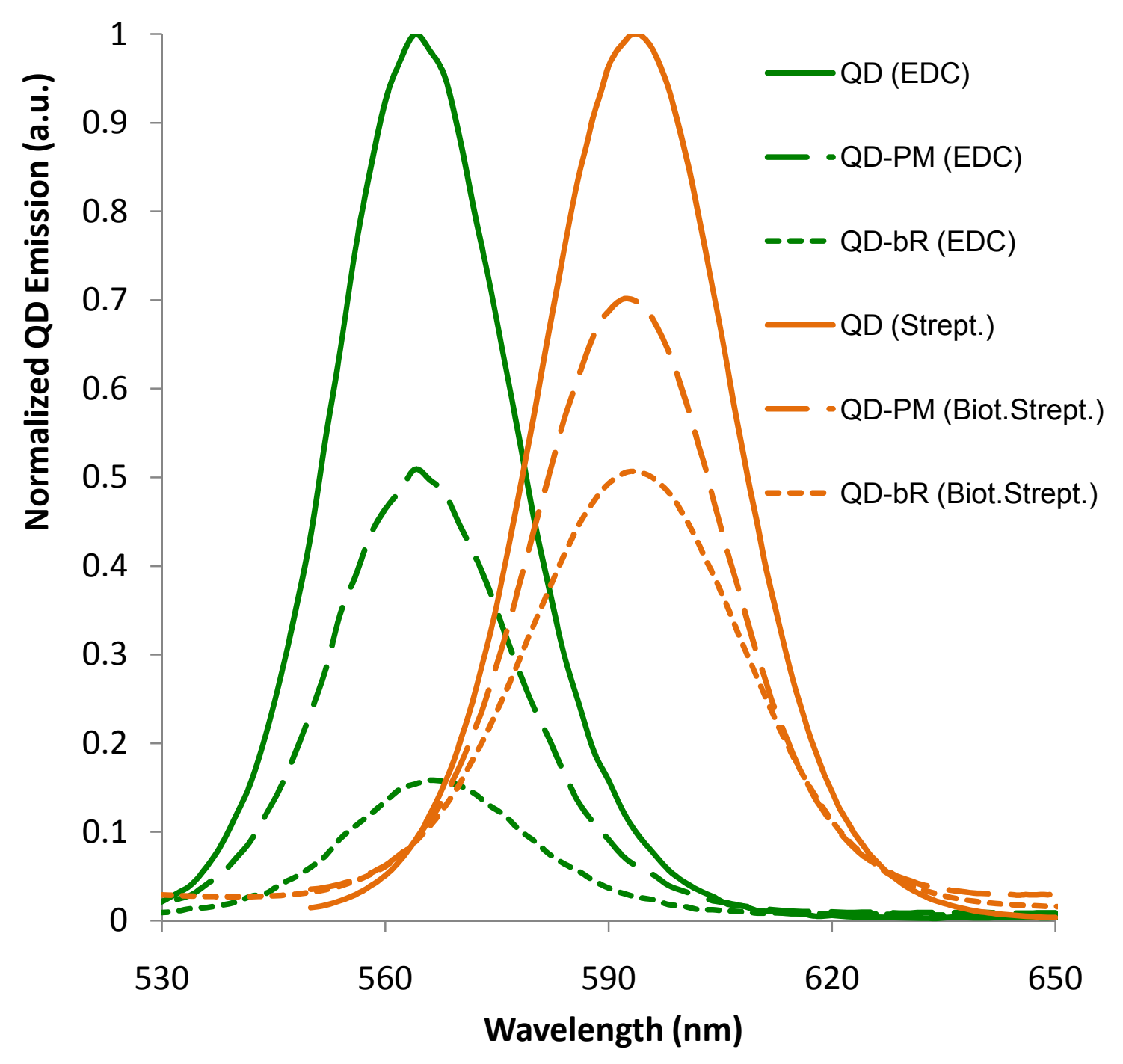

Figure 96. Comparison of EDC and Biotin-Streptavidin linkages of QD to bR, in both PM patch and monomer form.

Table 5. Comparison of theoretical FRET efficiency of $565 \mathrm{~nm}$ and $595 \mathrm{~nm}$ emission QDs linked to bR.

\begin{tabular}{|l|c|c|}
\cline { 2 - 3 } \multicolumn{1}{c|}{} & \multicolumn{2}{c|}{ FRET Efficiency (E) } \\
\cline { 2 - 3 } \multicolumn{1}{c|}{} & $\mathbf{5 6 5 n m ~ Q D}$ & $\mathbf{5 9 5 n m ~ Q D ~}$ \\
\hline $\mathbf{3 . 5 n m}$ & $99.07 \%$ & $99.01 \%$ \\
\hline $\mathbf{8 . 5 n m}$ & $34.17 \%$ & $32.79 \%$ \\
\hline
\end{tabular}


It can be seen in Figure 96 that a reduction in separation distance between the QD core and bR retinal resulted in measurable increases in the bR-QD FRET coupling relationship. Theoretical calculations confirm minimal alteration in FRET efficiency between $565 \mathrm{~nm}$ and $595 \mathrm{~nm}$ emission QDs, thus the differences in QD emission were isolated to the separation distance. A comparison of bR-QD FRET efficiency versus separation distance is shown in Table 6.

Table 6. Comparison of experimental bR-QD FRET efficiency based on separation distance.

\begin{tabular}{|c|c|c|}
\cline { 2 - 3 } \multicolumn{1}{c|}{} & \multicolumn{2}{c|}{ FRET Efficiency (E) } \\
\cline { 2 - 3 } \multicolumn{1}{c|}{} & PM & bR monomer \\
\hline $\mathbf{3 . 5 n m}$ & $49 \%$ & $88 \%$ \\
\hline $\mathbf{8 . 5 n m}$ & $32 \%$ & $49 \%$ \\
\hline
\end{tabular}

For both the PM fragments and bR monomers, a closer proximity to the QD core achieved a greater degree of FRET coupling. Specifically for the PM fragment, lowering the separation distance from $8.5 \mathrm{~nm}$ to $3.5 \mathrm{~nm}$ increased the FRET efficiency by $17 \%$, while in the case of bR monomers the FRET efficiency was increased by 39\%. A larger magnitude adjust to FRET efficiency is seen in the bR monomer system, which more closely resembles the change expected from the theoretical model. Additionally it is possible that more binding spots were available on the carboxyl-coated QDs, used for the $3.5 \mathrm{~nm}$ separation, relative to the streptavidin-coated QDs, used for the $8.5 \mathrm{~nm}$ separation. This factor would be less apparent with the PM fragments since the effects of steric hindrance would likely be the limiting factor controlling the amount of bR binding to the QDs. 


\subsection{QD LIFETIME DECAY ANALYSIS WITH EDC-LINKED BR-QD PAIRS}

To conclude that a FRET coupling relationship between the QD core and bR retinal is the primary cause of QD emission reduction, as opposed to other absorptive or concentration effects, the excited state lifetime of the QD was analyzed. Lifetime decay works by exciting the QDs with a sub-nanosecond laser pulse to excite the electrons to their excited state. As these excited electrons relax to the valence band, they release energy in the form of a photon. A single photon counting spectrometer was used in line with the excited QDs to monitor the time progression of photon release following laser excitation. This allwed the rate at which each excited QD electron relaxes back to its original state to be monitored. The amount of time it takes for the QD emission to reach 1/e (36.79\%) of its initial emission is termed the QD excited state lifetime.

In a FRET coupled system the number of QD electrons available to produce photons will be diminished. This is due to a portion of the excited QD electrons tunneling to the acceptor molecule's conduction band, as illustrated for a bR-QD FRET coupled system in Figure 97. Thus when normalized to the QD control, a FRET coupled QD system would have the same initial emission peak, but would reach its $1 / \mathrm{e}$ emission sooner. This is due to a portion of the photon-producing excited electrons being transferred to the overlapping energy band in the acceptor molecule, thus reducing the amount of QD photons released over time and ultimately reducing the QD excited state lifetime. Normal photon absorptive effects have no effect on the QD lifetime since it would reduce the photon count equally over the entire timescan and not affect the rate. Thus utilizing the excited state lifetime technique for a bR-QD system allows the energy transfer relationship to be isolated from other quenching phenomenon and concentration effects.

The setup where this work was performed is located at the Army Research LaboratoryAdelphi, supervised by Dr. Gregory Garret. The pump laser was an optical parametric amplifier (OPA) pumped by a $250 \mathrm{kHz}$ regenerative amplified Ti:Sapphire laser. The pulses were doubled using a nonlinear crystal [63] to give $\sim 10$ fs pulses at $375 \mathrm{~nm}$. The 
laser was focused to a spot size of $\sim \mu \mathrm{m}$ diameter, which provided incident fluence of $35.6 \mu \mathrm{J} / \mathrm{cm}^{2}$, not accounting for losses due to the quartz cuvette. This laser energy approximated to an optical power of $175 \mathrm{uW}$. The collected photoluminescence was dispersed using a Triax 320 spectrometer using a 300 groove $/ \mathrm{mm}$ grating. The bandwidth of the collected signal of the time correlated single photon counting (TCSPC) data was approximately $20 \mathrm{~nm}$.

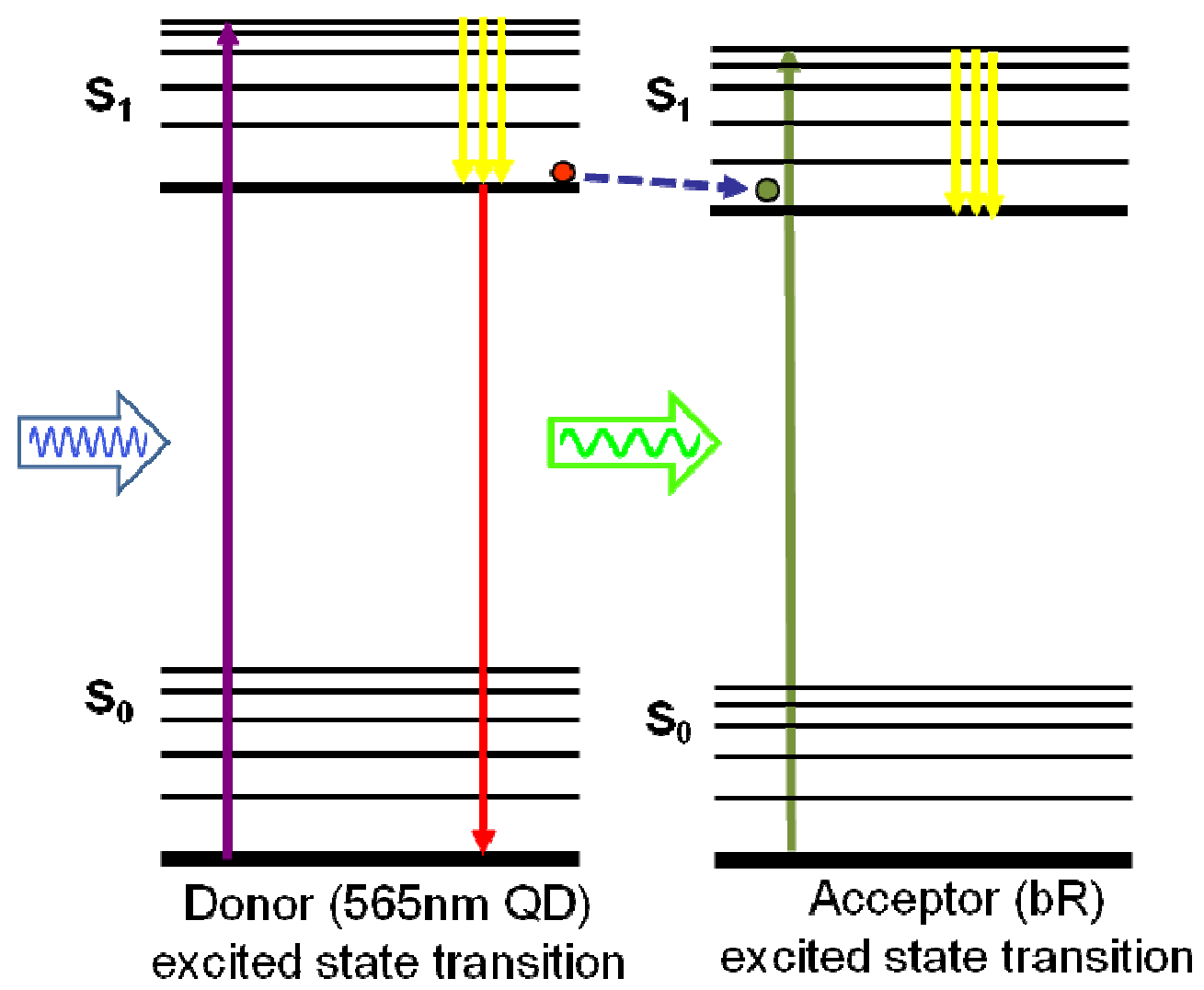

Figure 97. Jablonski diagram showing FRET between a donor and an acceptor molecule. The purple arrow shows QD absorption, yellow arrow shows vibrational relaxation, red solid arrow shows fluorescence. Blue arrow shows non-radiative energy transfer from the donor QD to acceptor biomolecule.

The fluorescence lifetimes were measured at the QD emission maxima of $565 \mathrm{~nm}$ and monitored the electron transfer characteristics of the QD control and QD when bR (in PM fragment form) was directly linked via a zero-length EDC linker. For a control, the 
carboxyl QDs were run through the EDC linkage protocol without adding any bR for binding. The excited state lifetime of the control QDs is displayed in Figure 98. Plotting the QD intensity on a log-scale should provide a linear curve, unless multiple QD lifetimes are present in the system. In addition the control QD lifetime intensity is plotted on a linear-scale, as displayed in Figure 99. Likewise the QD excited state lifetime of a QD-bR (PM patch form) pair linked via EDC are displayed in log and linear forms in Figure 100 and Figure 101, respectively.

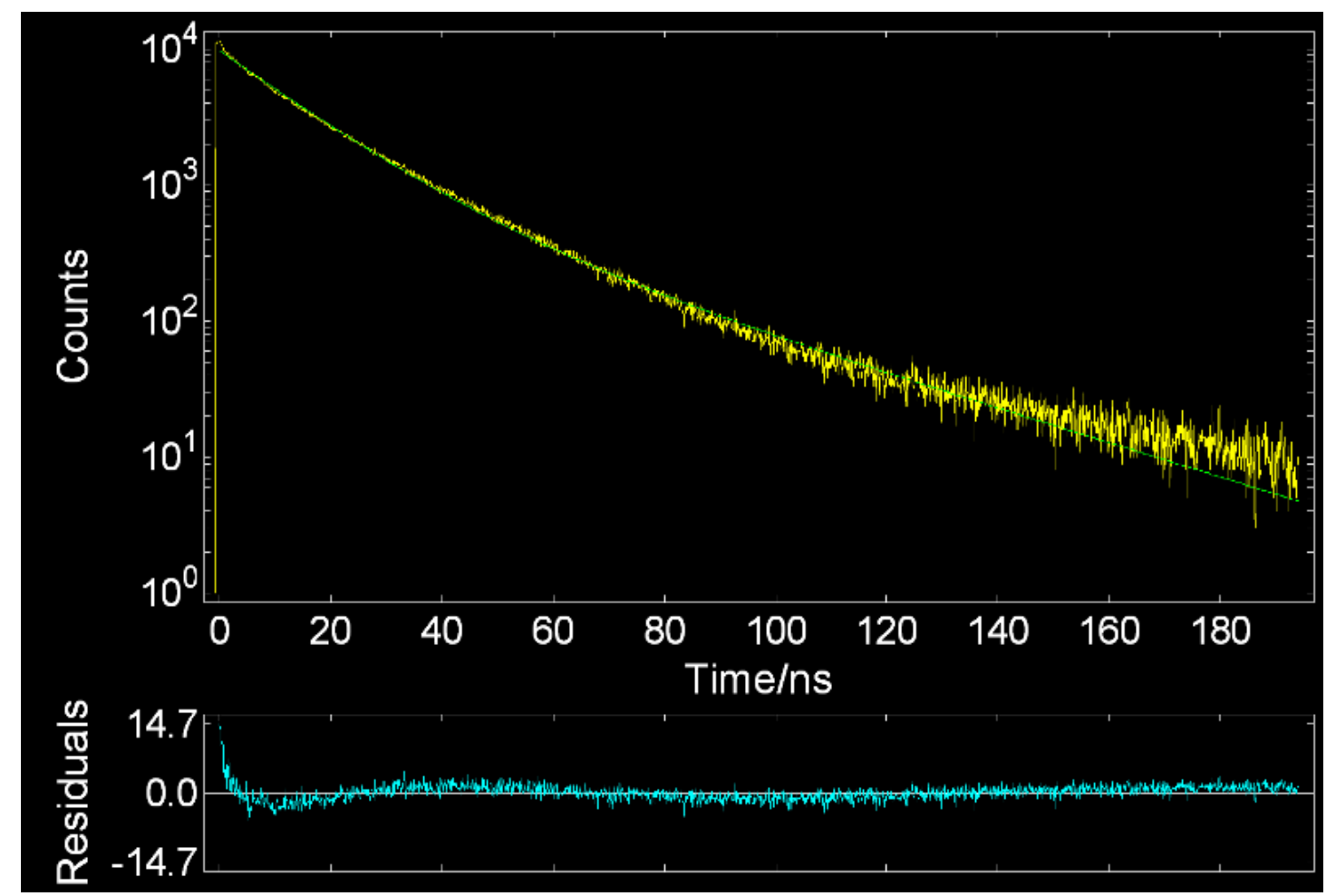

Figure 98. QD excited state lifetime decay of carboxyl QD control with intensity in log-scale. 


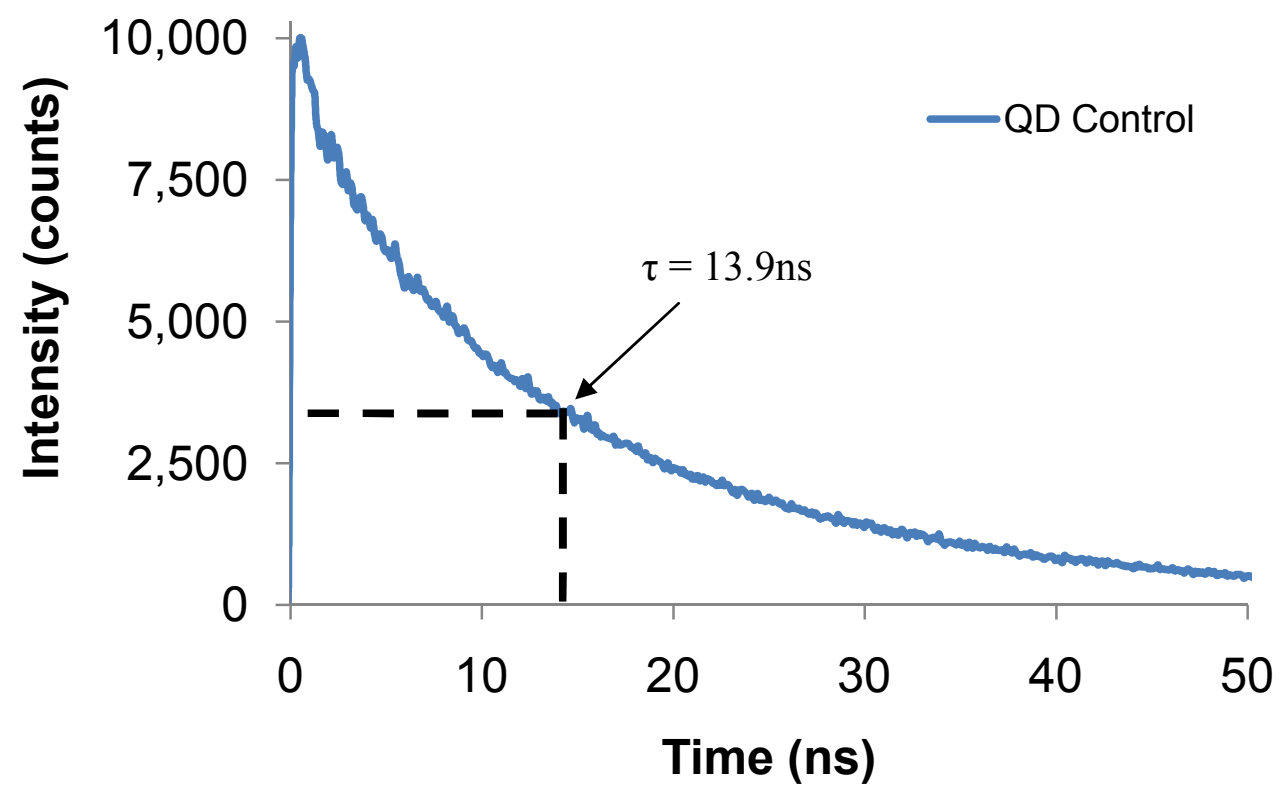

Figure 99. Excited state lifetime decay of QD control. Dashed lines mark the 1/e intensity location.

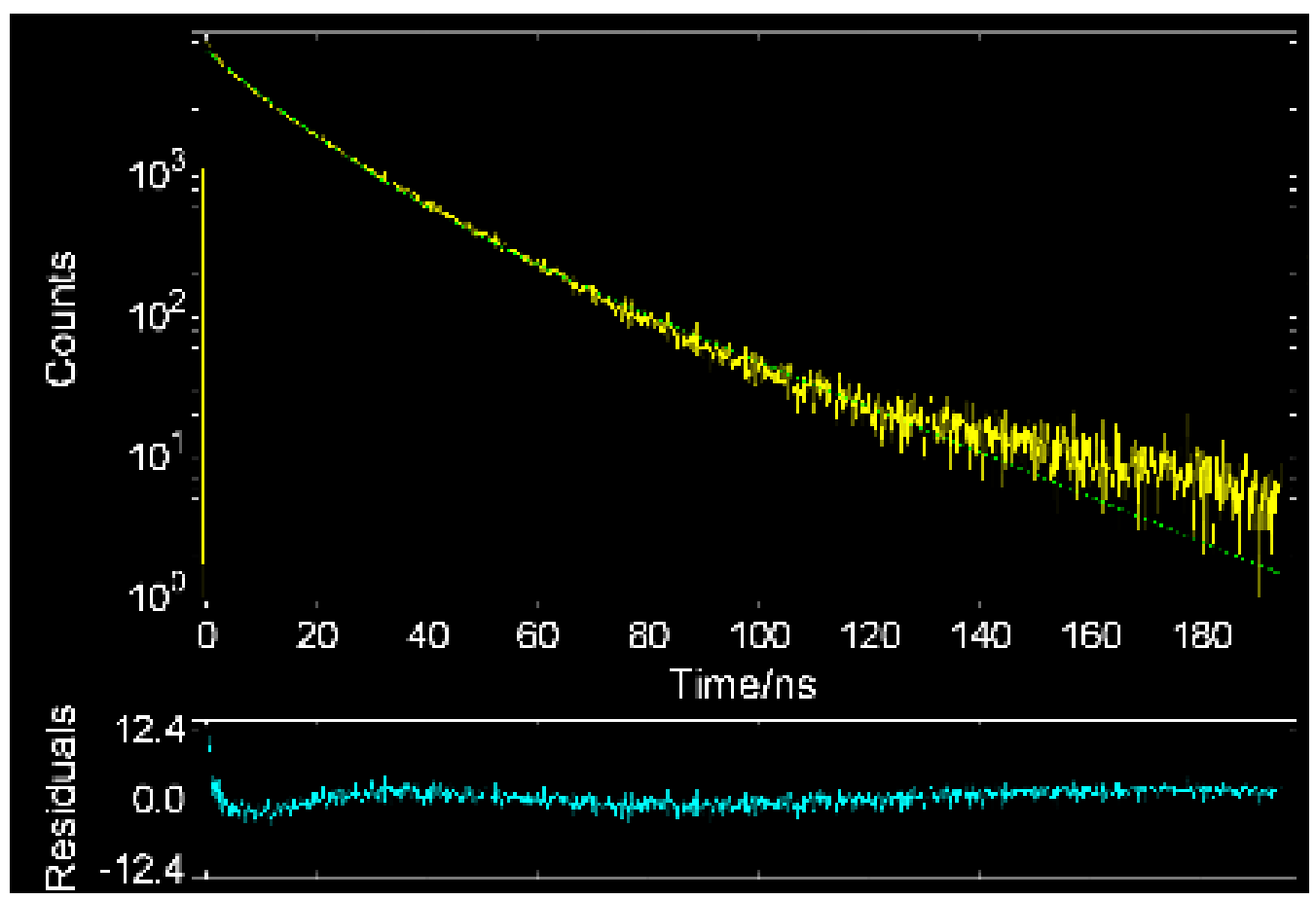

Figure 100. QD excited state lifetime decay of an EDC linked PM-QD system with intensity in logscale. 


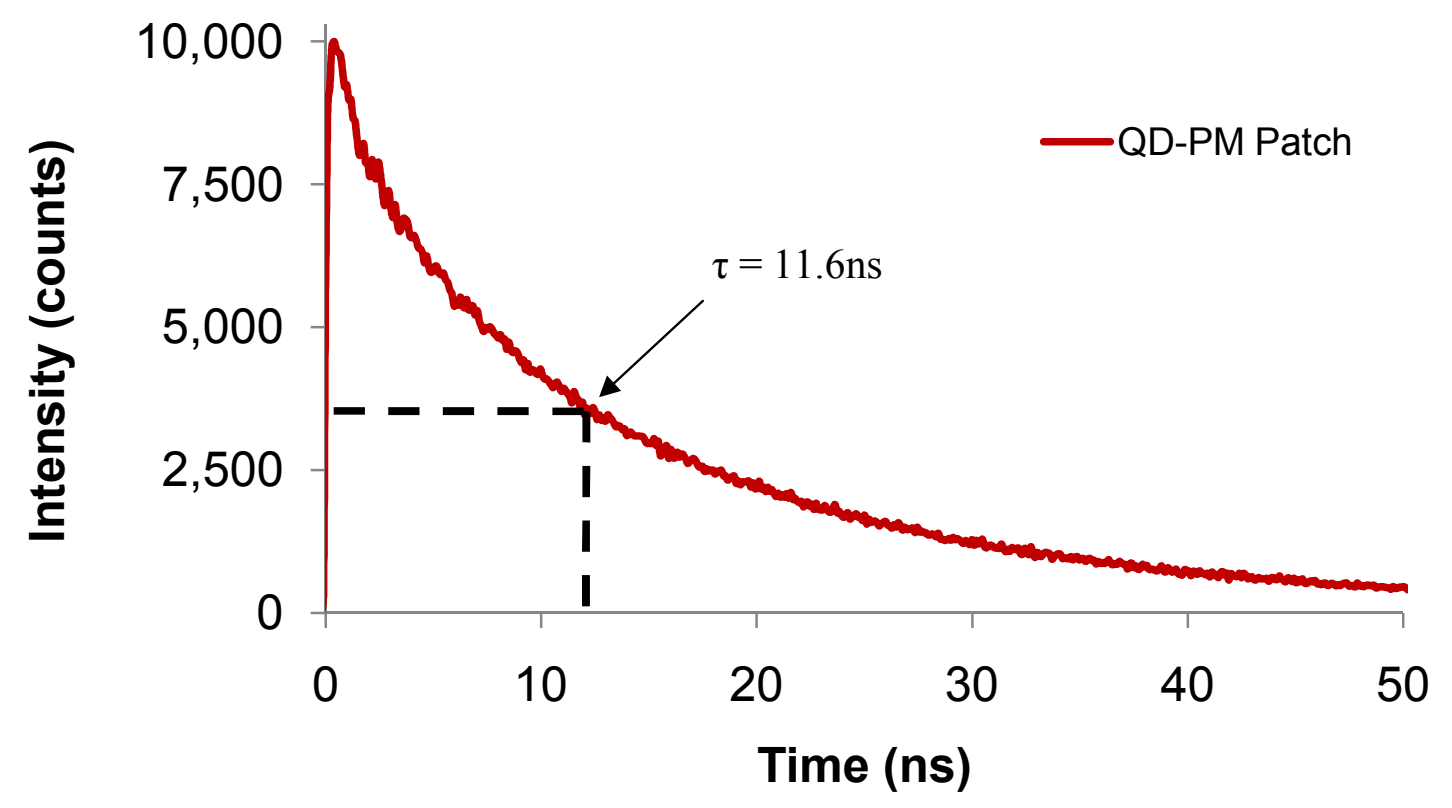

Figure 101. Excited state lifetime decay of EDC linked QD-bR (PM patch form). Dashed lines mark the $1 / \mathrm{e}$ intensity location.

In the excited state lifetime analysis of the QD control, as displayed in Figure 98, it can be noted that the decay rate on a $\log$ (intensity) scale is not perfectly linear. This implies that the QD control has multiple decay lifetimes. The lifetime computation from the data confirms this, revealing a bi-exponential decay with lifetimes of $\tau_{1}=13.93 \mathrm{~ns}$ and $\tau_{2}=34.60 \mathrm{~ns}$. The magnitude of lifetime contribution varied, however, with $\tau_{1}$ contributing $73 \%$ of the lifetime component and $\tau_{2}$ with $27 \%$. Applying a weighted-average to the two lifetime components yields a QD control lifetime of $19.51 \mathrm{~ns}$.

The excited state lifetime analysis of the EDC linked QD-br (PM patch form) is displayed in Figure 100 and, likewise, has a bi-exponential decay rate. The lifetime computation from the data reveals lifetimes of $\tau_{1}=11.58 \mathrm{~ns}$ and $\tau_{2}=27.41 \mathrm{~ns}$. The magnitude of lifetime contribution varied, however, with $\tau_{1}$ contributing $59 \%$ of the lifetime component and $\tau_{2}$ with $41 \%$. Applying a weighted-average to the two lifetime components yields a QD control lifetime of $18.07 \mathrm{~ns}$. 
Figure 99 and Figure 101 show the excited state decay in linear intensity units for the QD control and QD-PM patch, respectively. Analysis of the QD excited state decay rate at 1/e intensity reveals lifetimes similar to the first lifetime component computed above. These lifetimes were determined to be 13.90ns for the QD control and 11.60ns for the EDC linked QD-PM system. The excited state studies showed a definitive decrease in QD lifetime when bound in close proximity to the bR molecule. This data suggests that the second lifetime component apparent in each bi-exponential decay rate had a smaller effect, and could be due to molecular configuration changes of the bR during the experiment which could ultimately alter the separation distance between the bR retinal and QD core. It is possible that the non-photonic energy received by the bR retinal induced the proton pumping action. The changes in bR conformation due to proton pumping could have been a source of the increased secondary lifetime component, as the change in configuration would alter the FRET coupling relationship [84]. Therefore the initial lifetime recorded was considered the primary lifetime decay rate for this system. Specifically the results suggest that binding a bR molecule, in its PM patch form, within $3-4 \mathrm{~nm}$ of the QD results in a 2.44ns decrease in QD lifetime due to electron transfer to the bR molecule.

As with previous QD FRET coupling studies with PM, it is suggested that the large macromolecular form of the PM fragment inhibits adequate bR-QD linkage formation and ultimately reduces the degree of FRET coupling. To circumvent the potential FRET issues associated with PM fragments, the fragments were delipidated to attain bR monomers. The process for preparing a QD-bR monomer coupled system with an EDC linker was described in previous sections.

The fluorescence lifetimes of the QD-bR monomer system were measured at the QD emission maxima of $565 \mathrm{~nm}$. The excited state lifetime of this system is displayed in Figure 102 and is additionally plotted in linear scale in Figure 103. To give an overall excited state lifetime comparison, the QD control, QD-PM patch, and QD-bR monomer systems are plotted together in Figure 104 and Figure 105. 


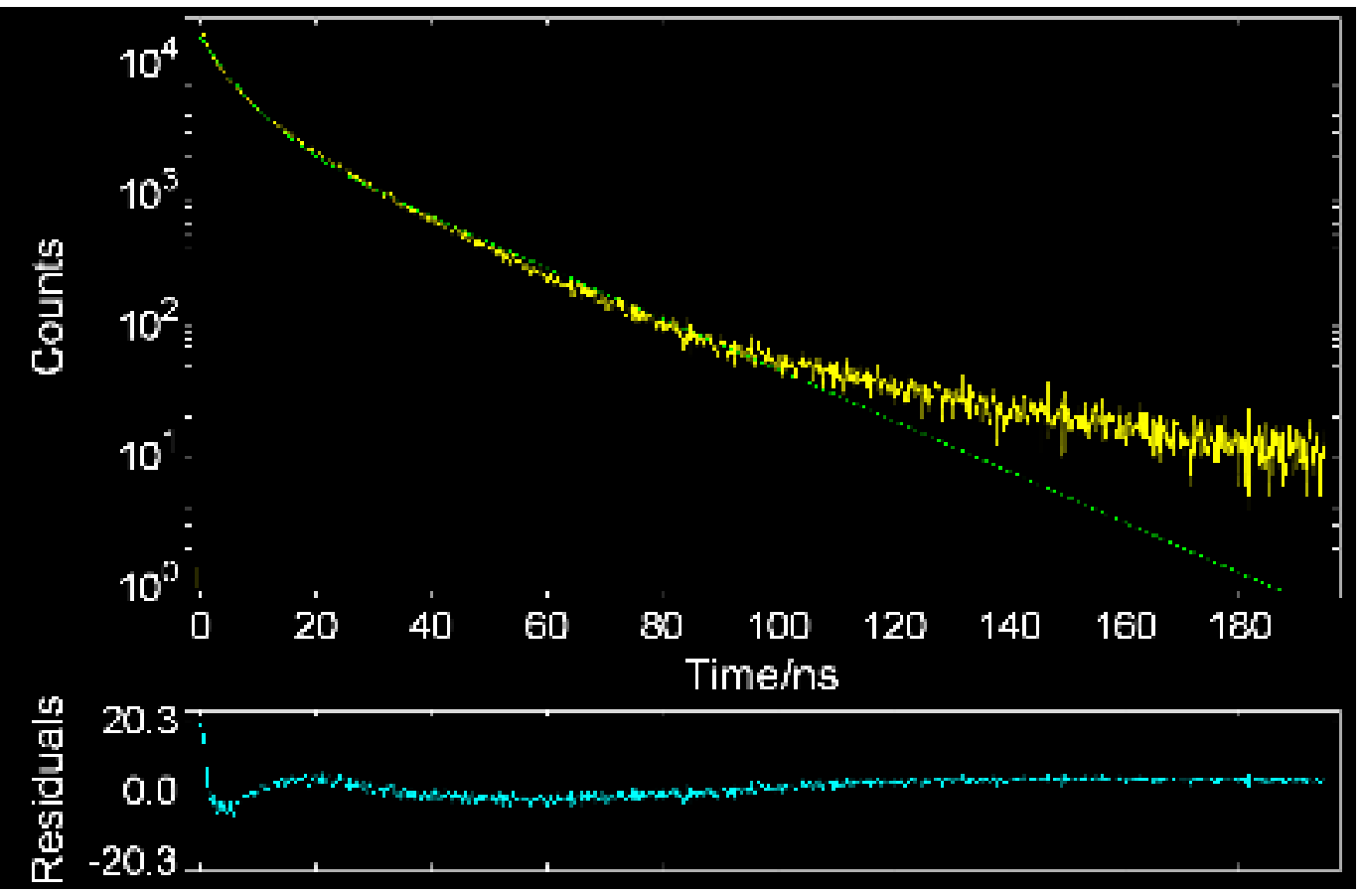

Figure 102. Excited state lifetime decay of $565 \mathrm{~nm}$ QD emission in a QD-bR monomer EDC linked system.

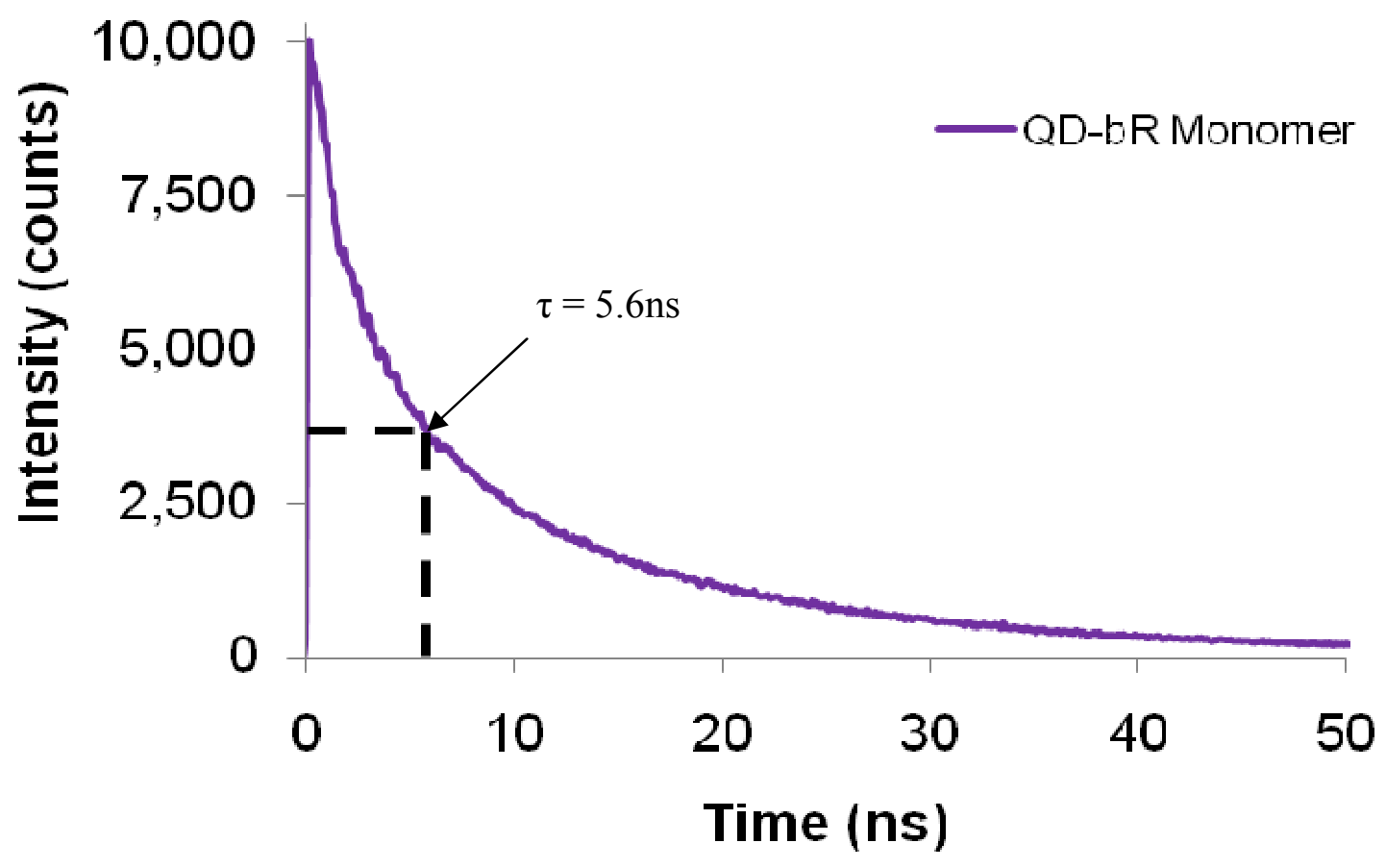

Figure 103. QD excited state lifetime of a QD-bR monomer EDC linked system plotted on a linear scale. 


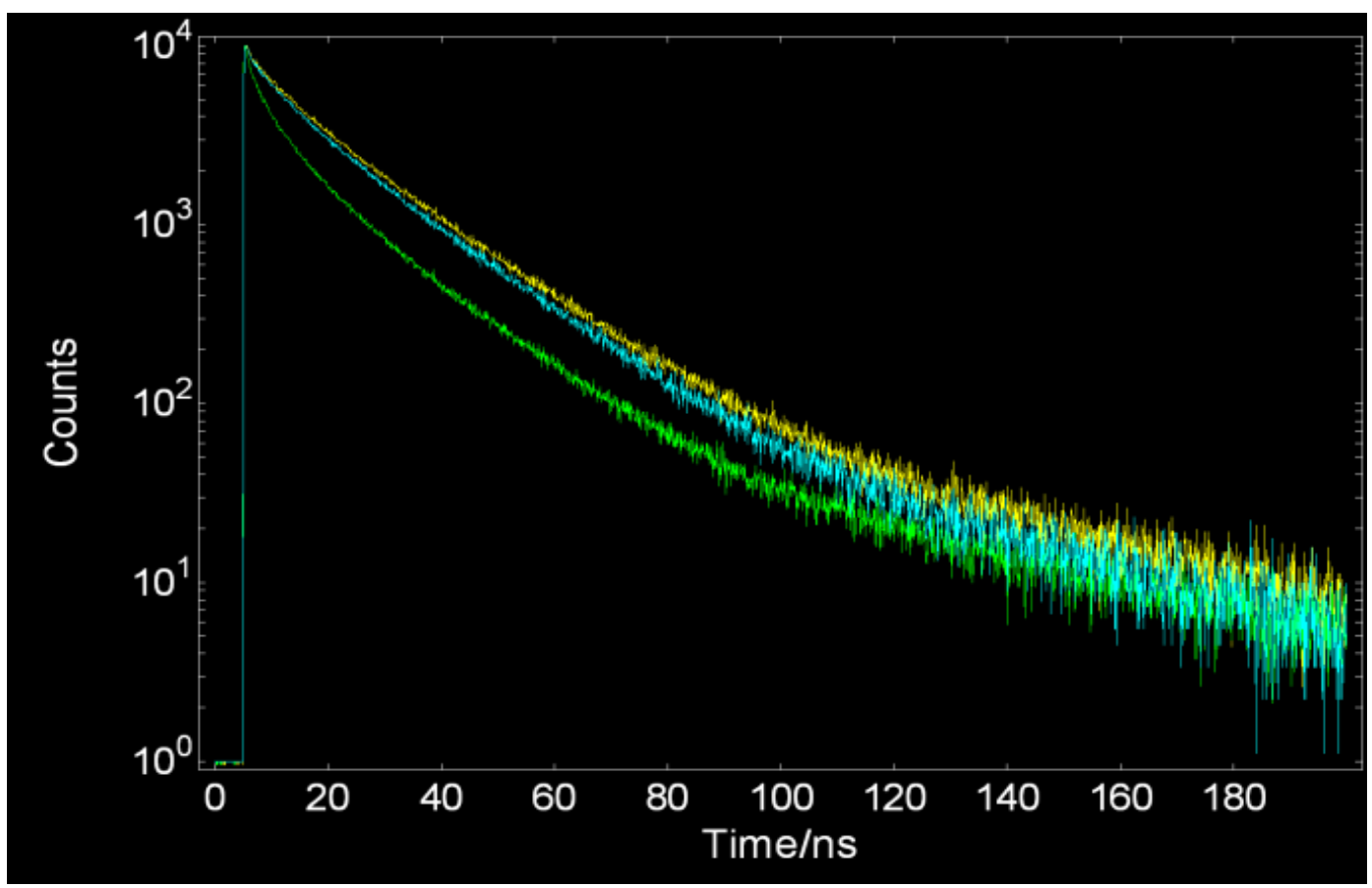

Figure 104. Overall QD excited state lifetime comparison, with the QD control (yellow), QD-PM patch (light blue), and QD-bR monomer (green) pairs linked with EDC.

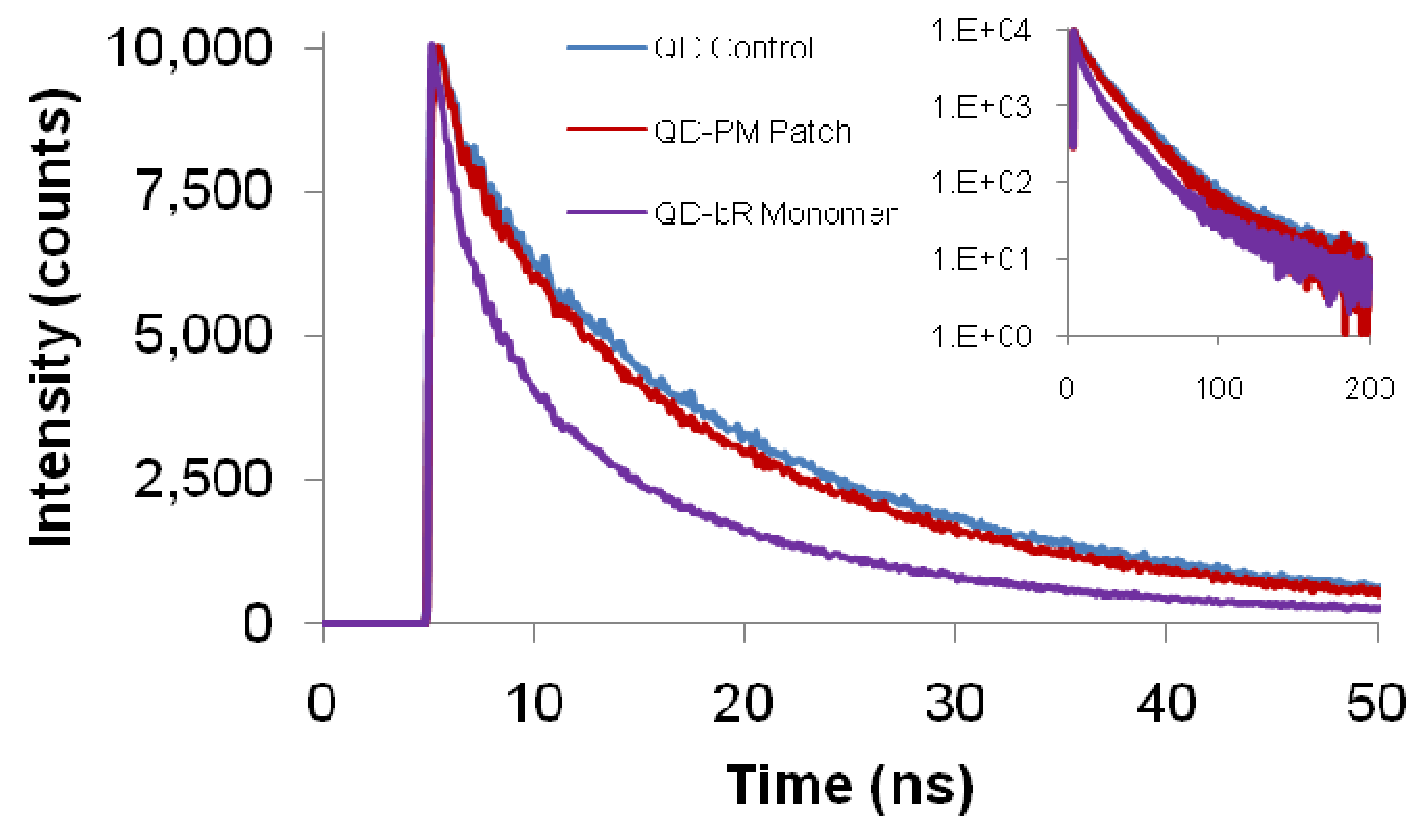

Figure 105. QD lifetime decay when linked to $b R$ in PM patch form ( $\tau=11.58 \mathrm{~ns})$ and $b R$ monomer form ( $\tau=5.61 \mathrm{~ns})$ compared to the QD only control ( $\tau=13.93 \mathrm{~ns})$. Inset displays identical QD excited state lifetime decay data with intensity (counts) in Log-scale. 
In the excited state lifetime analysis of the QD control, as displayed in Figure 102, it was noted that the decay rate on a $\log$ (intensity) scale was not perfectly linear. This implies that the EDC-linked QD-bR monomer hybrid has multiple decay lifetimes. The lifetime computation from the data confirms this, revealing a bi-exponential decay with lifetimes of $\tau_{1}=5.61 \mathrm{~ns}$ and $\tau_{2}=22.39 \mathrm{~ns}$. The magnitude of lifetime contribution varied, however, with $\tau_{1}$ contributing $42 \%$ of the lifetime component and $\tau_{2}$ with $58 \%$. Applying a weighted-average to the two lifetime components yields a QD control lifetime of $15.34 \mathrm{~ns}$. This data again suggests molecular configuration changes of the bR during the experiment which could ultimately alter the separation distance between the bR retinal and QD core. Analysis of the QD excited state decay rate at 1/e intensity in Figure 103 reveals a lifetime decay rate similar to the first lifetime component computed above of $5.61 \mathrm{~ns}$.

The overall comparison of the excited state lifetimes of the three tested systems in Figure 104 distinctly shows the high degree of electron transfer in the QD-bR monomer system. This distinction is more evident on the linear scale, as shown in Figure 105. The excited state studies show a definitive decrease in QD lifetime when bound in close proximity to the bR molecule in both the PM patch and bR monomer form. Specifically a lifetime decay rate decrease of $16.9 \%$ and $59.7 \%$ was seen compared to QD control for the PM patch and bR monomer systems, respectively.

The ability to form a FRET coupling relationship between the bR retinal and the QD core has a variety of applications ranging from biocomputing to photovoltaics. In the case of biocomputing, groups have studied the utility of bR's different conformation/absorption states to serve as a logic circuit with picosecond response time $[125,126]$. The direct electron transfer in a bR-QD FRET coupled system allows the QD absorption band to become directly coupled to the bR conformational states. This ultimately increases the optical range and the discernability of logic states for each $\mathrm{bR}$ molecules, expanding its applicability in biocomputing. For solar cell applications the capability enhancement again comes from the expanded energy absorption range provided by the QDs, except in this setup the transferred energy will provide more activation energy for the bR proton 
pumping cycle. Current work has demonstrated the superior capabilities of $b R$ as a solar cells with its natural absorption spectra $[32,127]$, thus the ability to expand bRs solar capture spectrum via the QD absorption band has the potential to greatly enhance bRs photoconversion efficiency.

For the proposed biosensing system, a bR-QD FRET coupling relationship provides a generic substrate with potential for extreme target sensitivity. Since the FRET coupling efficiency is directly dependent upon separation distance, a sudden change in this separation distance will alter the energy transfer relationship and QD photonic output. With the bR photovoltaic output directly dependent upon the energy received from the QD, either photonically or non-photonically, an instantaneous adjustment to the bR output will be seen with any alteration to the bR-QD FRET coupling relationship. This is particularly true if the $\mathrm{bR}-\mathrm{QD}$ separation distance is in the Forster radius region of $7.62 \mathrm{~nm}$, since this region displays the largest variability in FRET with sub-nanometer distance changes. The integration of a target-specific ligand around the QD or in between the bR and QD layers could provide sufficient alteration to the bR-QD FRET coupling relationship upon target binding to alter the bR photovoltaic output, and thus give the biosensing capability.

\subsection{DRY FILM BR-QD FRET}

Previous work has demonstrated that fluorescence resonance energy transfer coupling can be achieved between quantum dots and bacteriorhodopsin in a liquid suspension. For sensor development, however, it is necessary to control the degree of FRET coupling between $b R$ and QDs in a dried state.

Using fluorescence microscopy techniques, the phenomena of QD quenching when dried on top of a bR film was imaged. In Figure 106, it can be seen that the dried QDs on top of the bR (upper left) have a fluorescence emission far less than the dried QDs on the 
glass slide (lower right). It is believed that the reduction in fluorescence emission is due to FRET coupling between the dried QDs and bR film.

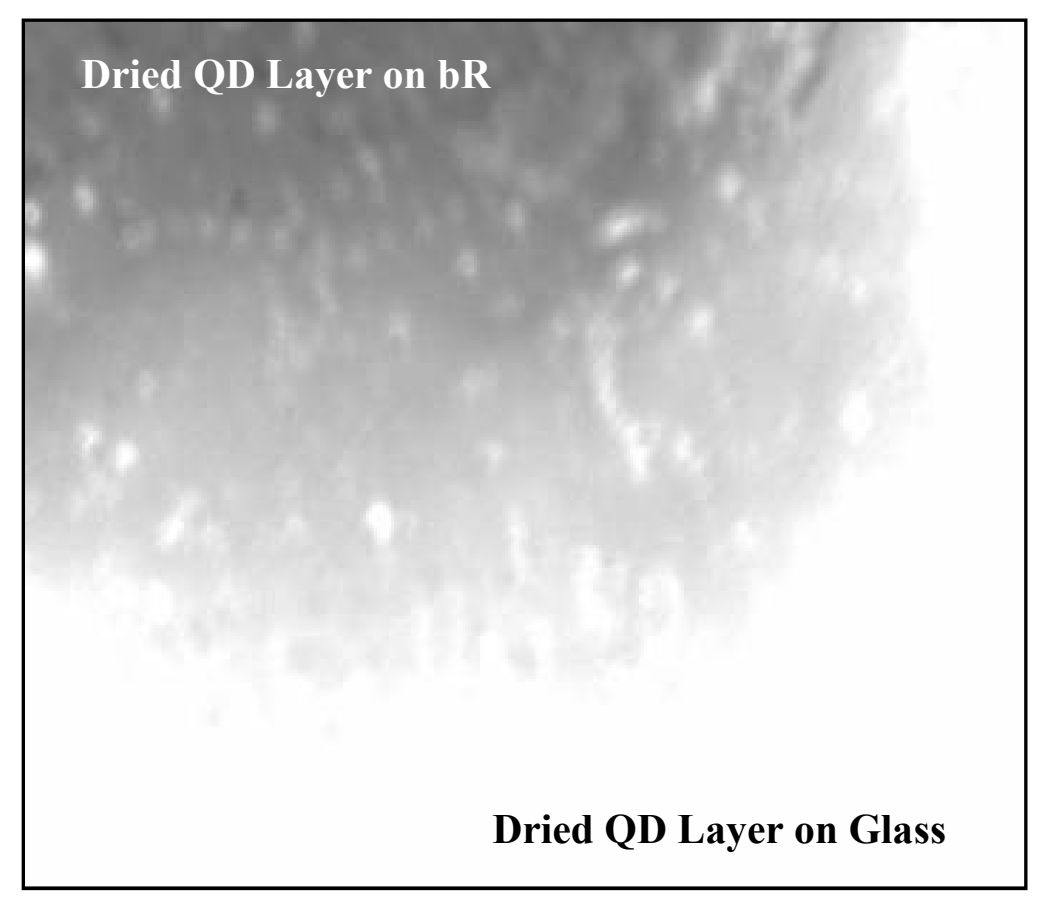

Figure 106. Fluorescence emission image of dried QD layers on top of bR film and glass

To quantify the degree of FRET coupling when QDs are deposited and dried directly on top of dried bR, the dry sample plate reader on the Hitachi F-7000 spectrofluorimeter was used. Additionally, since one potential bR electrode design involves sputtering a $5 \mathrm{~nm}$ layer of gold on top of the bR to serve as the counter-electrode, the FRET coupling of a gold substrate with QDs was also evaluated.

For this study the quenching effects of four different substrates was analyzed:

- Blank Glass Slide (“Glass”) - Control

- Glass slide with $5 \mathrm{~nm}$ thick gold sputtered on top ("Glass - Gold")

- Glass slide with $25 \mu \mathrm{L}$ of $5 \mathrm{mg} / \mathrm{mL}$ dried bR on top ("Glass - bR")

- Glass slide with $25 \mu \mathrm{L}$ of bR $(5 \mathrm{mg} / \mathrm{mL})$ and $5 \mathrm{~nm}$ thick gold sputtered on top of the bR ("Glass - bR - Gold") 
The wavelength of interest is $\sim 570 \mathrm{~nm}$ since this is approximately the peak emission for the Evident CdSe Core QDs used in this study. For a baseline, the fluorescent emission of the substrates before the addition of QDs was obtained. A total of $0.5 \mu \mathrm{L}$ of 44.63nmol/ml CdSe QD's suspended in toluene was deposited in the center of each sample, yielding a total deposition of $2.23 \times 10^{12}$ QDs. The estimated $2 \mathrm{~mm}$ spot size of deposited QDs should create a film approximately 28 QD layers thick. The fluorescent emission of the sample was analyzed once the QDs had dried on the substrate for 5 minutes at room temperature. The fluorescent emission from the QDs when dried on each substrate is shown in Figure 107. These results were normalized with respect to their background noise without QDs present.

The results shown in Figure 107 were analyzed assuming that no QD quenching was present on the blank glass slide with deposited QDs, thus the control study. With respect to the control, all the substrates displayed QD quenching to some degree. The degree of QD quenching and the maximum peaks for each substrate are listed in Table 7.

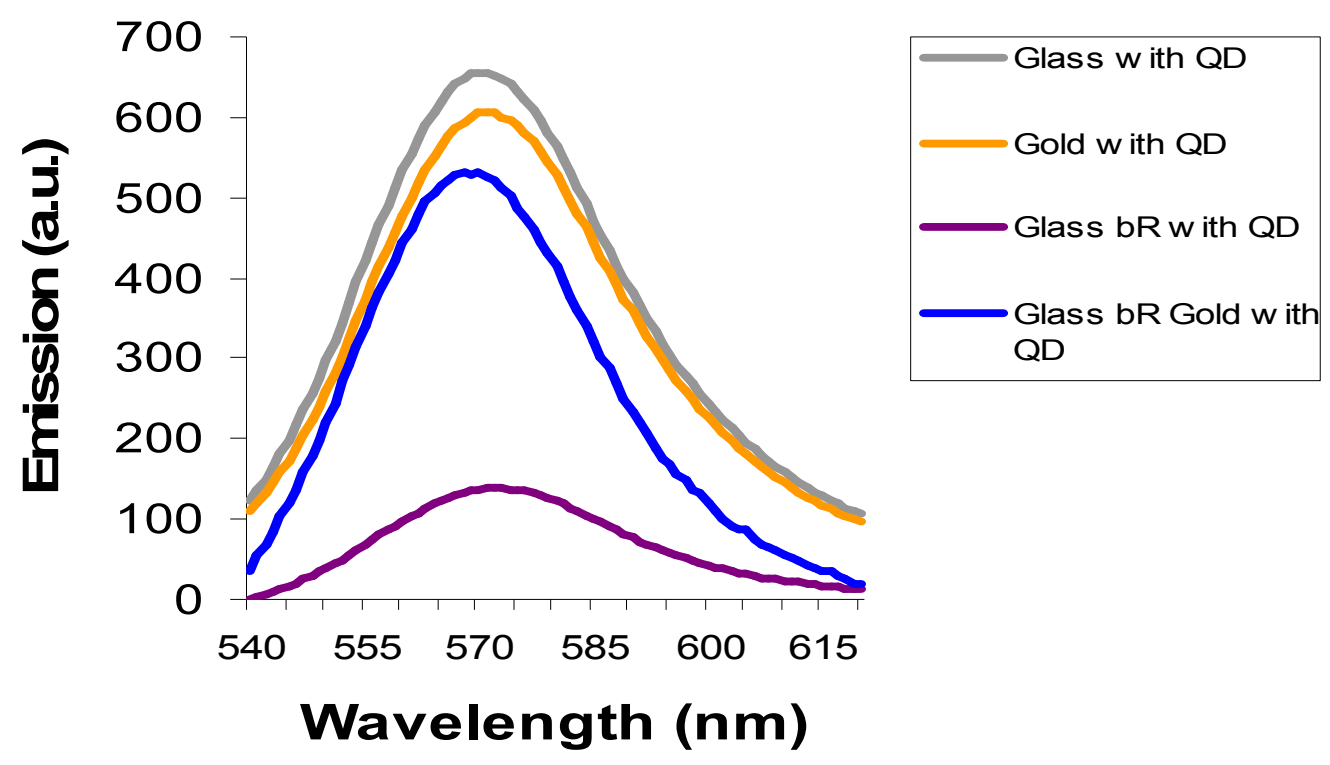

Figure 107. QD emission vs. wavelength on varying substrates 
Table 7. Peak emission and QD quenching levels vs. substrate

\begin{tabular}{|c|c|c|c|}
\hline Substrate & $\begin{array}{c}\text { Peak Wavelength } \\
(\mathbf{n m})\end{array}$ & $\begin{array}{c}\text { Peak Emission } \\
(\mathbf{n m})\end{array}$ & FRET Efficiency (E) \\
\hline Control (Glass) & 570 & 655.4 & 0 \\
\hline Glass - Gold & 571 & 606.2 & $7.51 \%$ \\
\hline Glass - bR & 572 & 138.7 & $78.84 \%$ \\
\hline Glass - bR - Gold & 570 & 530.1 & $19.12 \%$ \\
\hline
\end{tabular}

The initial results suggest that there was a slight degree of QD quenching associated with the $5 \mathrm{~nm}$ gold layer. The highest degree of FRET quenching was seen when the QDs were deposited directly on top of the bR film. This was expected due to the high degree of overlap between the QD emission and the bR absorption. The direct contact between the QDs and bR resulted in a 78.84\% reduction in QD emission. The emission from the QDs was not completely quenched due to the fact that there was not just a single monolayer of QDs on top of the bR film, but several layers. Therefore the QD layers in close contact with the bR might have been undergoing a high degree of FRET, while the top-most QD layers were less affected. It was also noted that the fluorescent emission of the samples once the QDs were dried on top varied over time, as is shown in the 15 minute time-scan of 570nm emission shown in Figure 108. 


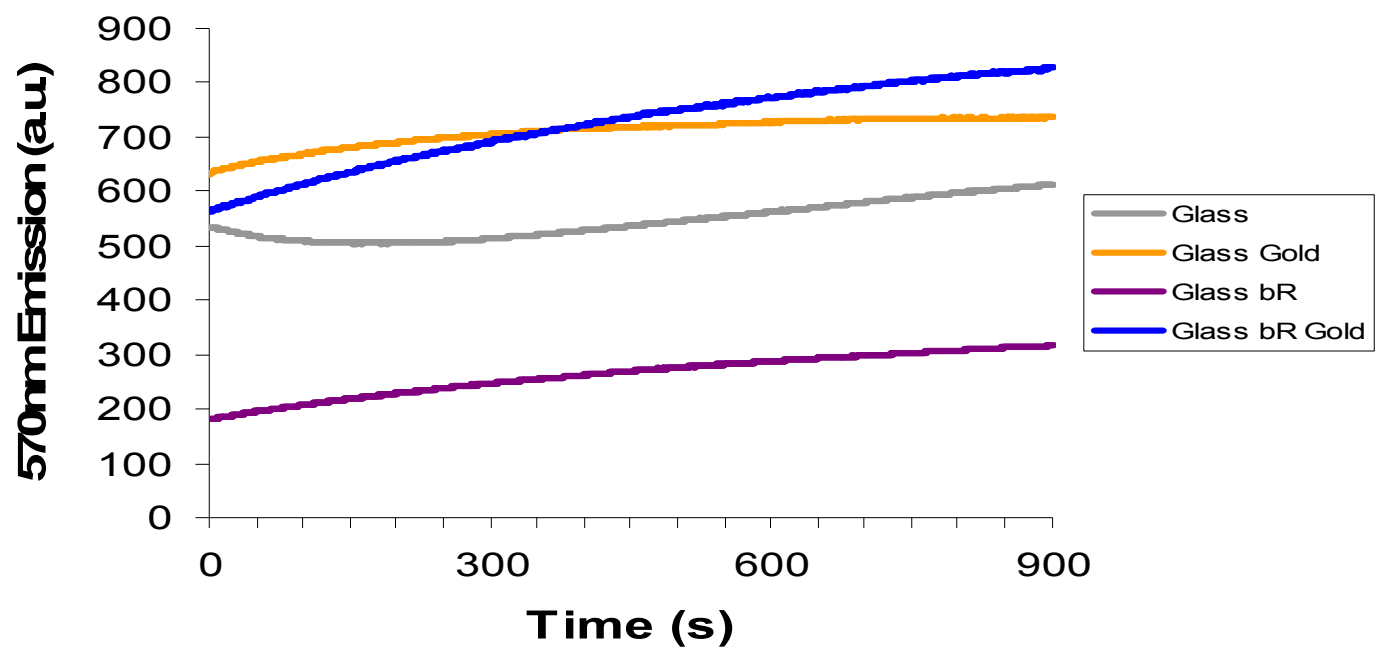

Figure 108. Timescan of 570nm QD emission vs. time on varying substrates

From the time-scan it can be noted that, in general, the fluorescence intensity of the QD increases over time until it gradually levels off. The increase in fluorescence emission was likely due to a phenomenon known as photo-annealing. The photo-annealing effect has been shown to correct discontinuities in the QD and increase optical output. According to the literature, another process is also occurring simultaneously. The QDs used in this study were CdSe colloidal semiconductors. There wasn't a protective coating, such as $\mathrm{ZnS}$ or a polymer, to protect the dot from the environment. Therefore oxidation of the dot will eventually result in a blue-shift in the emission and eliminate the output all together. In the current study, however, there are multiple layers of dried QDs on the substrate and the inner layers might have been shielded from this destructive effect. From Figure 108 it appears that the QDs on a glass substrate experienced a greater degree of oxidation due to the sharp initial decrease in emission prior to the photo-annealing effect.

To reduce the thickness of the QD films and to further clarify FRET coupling in dried bR-QD films, experiments were conducted with reduced amounts of QDs in addition to using bleached $\mathrm{bR}$ as a substrate. The bleached form of bacteriorhodopsin is where the retinal has been removed. With the removal of the retinal, the technical name of the protein then becomes bacterioopsin (bO). The retinal is responsible for the light 
absorption around $570 \mathrm{~nm}$ and the proton pumping cycle, therefore without the retinal all functional properties of $b R$ are lost. Additionally when bR serves as an acceptor in FRET, the retinal is where the donor's energy is transferred. Therefore the BO protein should be unable to participate in the FRET process.

One method to remove the $\mathrm{bR}$ retinal is through continuous illumination with high intensity light. Depending on the light intensity, the bleaching process can take several days. To increase the speed of this process, the $\mathrm{pH}$ of the PM solution can be increased and hydroxylamine can be added. For these studies a $10 \mathrm{mg} / \mathrm{ml}$ PM solution was used. Hydroxylamine (MW 69.5) was added to the solution at $0.3 \mathrm{M}$ and the $\mathrm{pH}$ was increased to 7.5. The solution was then subjected to continuous high intensity light illumination at $500 \mathrm{~mW} / \mathrm{cm}^{2}$. Measuring the amount of bleaching using the Nanodrop spectrometer revealed that a high degree of bleaching was achieved in less than 24 hours as shown in Table 8. Upon reaching the desired level of bleaching, the PM solution was pelleted at 40,000X and washed multiple times with ddI water to remove any residual hudroxylamine.

Table 8. Magnitude of bR bleaching with hydroxylamine and light illumination over time.

\begin{tabular}{|c|c|}
\hline Time (hours) & Bleaching (\%) \\
\hline 0 & $0.57 \%$ \\
\hline 0.5 & $9.62 \%$ \\
\hline 1 & $25.33 \%$ \\
\hline 18 & $78.00 \%$ \\
\hline
\end{tabular}

The experimental setup compared the dried film fluorescence emission of each of these types of QDs on top of a control (blank glass slide), a dried bR film $(5 \mu \mathrm{L}$ of $5 \mathrm{mg} / \mathrm{ml}$ $\mathrm{PM})$, and a dried bO film ( $5 \mu \mathrm{L}$ of $5 \mathrm{mg} / \mathrm{ml}$, bleached for 24 hours). Each of the QD samples was suspended in toluene at a concentration of $0.5 \mathrm{nmol} / \mathrm{ml}$. A total of $1 \mu 1$ of the $0.5 \mathrm{nmol} / \mathrm{ml}$ QD solution was applied to the desired substrate for each particular test, 
yielding a total deposition of $5 \times 10^{10}$ QDs. The estimated $3 \mathrm{~mm}$ spot size of deposited QDs would create a film no more than 1 QD thick, thus allowing virtually all of the QDs to participate in any FRET coupling system. The resulting fluorescent emission of the dried QD films versus substrate is shown in Figure 109.

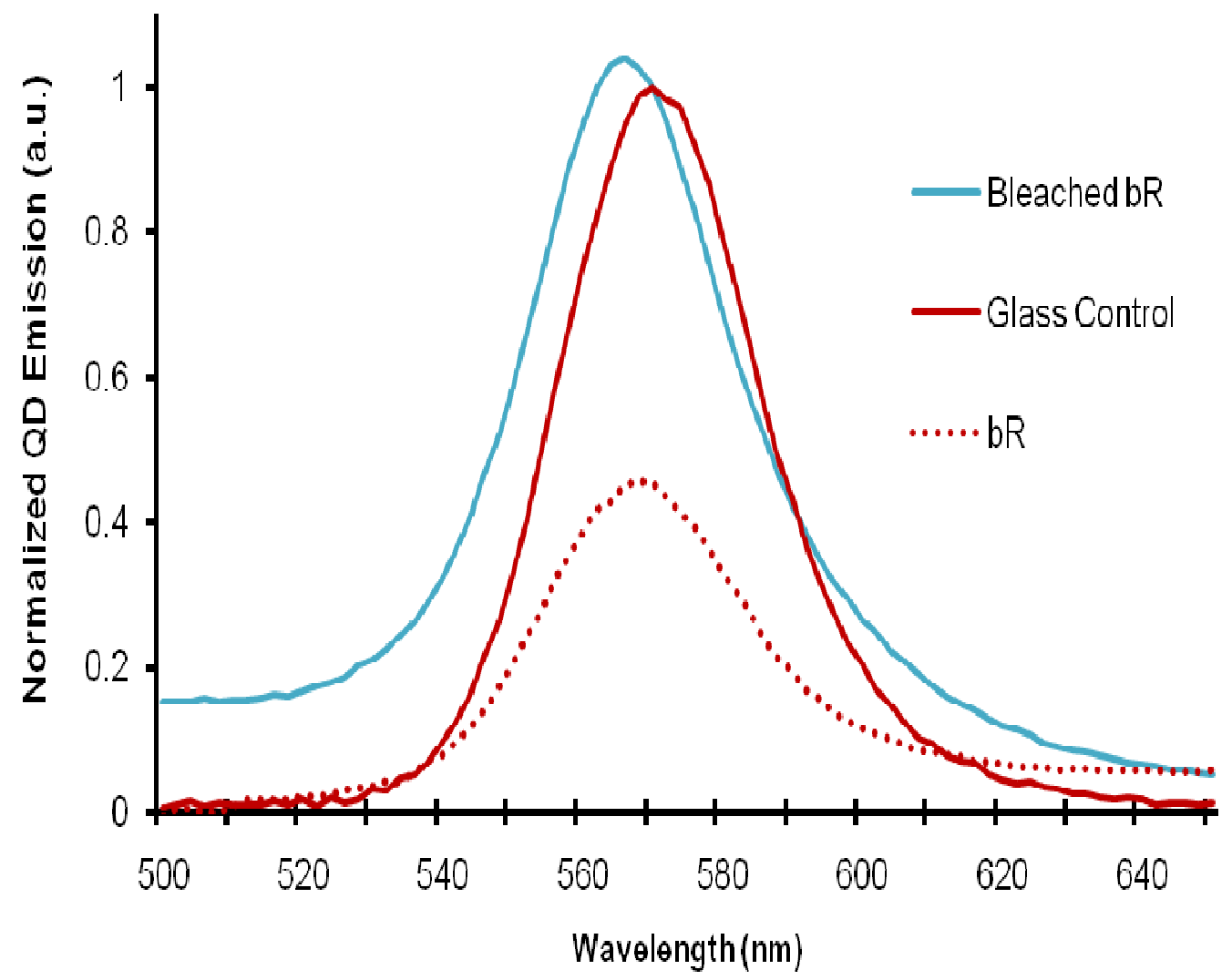

Figure 109. Control studying showing QD quenching effects of $b R$ and bO (bleached bR).

These results conclude that the bR retinal is the source of QD quenching in the experimental dried films. With this study confirming that the QD can FRET couple with the bR retinal in dried films, work was performed to analyze the effect of separation between the bR film and deposited QDs. This study compared the bR-QD FRET efficiency utilizing CdSe QDs with and without a polymeric shell. The QDs used in this study were purchased from Evident Technologies. Specifically the QDs were $566 \mathrm{~nm}$ CdSe core QDs and 575nm CdSe $/ \mathrm{ZnS}$ core-shell QDs. Estimates of the QD parameters 
were a $3.2 \mathrm{~nm}$ diameter CdSe core, a $1 \mathrm{~nm}-1.5 \mathrm{~nm}$ thick $\mathrm{ZnS}$ shell, and $2 \mathrm{~nm}$ amine capping agent. This led to an estimated diameter of 5.2nm for the CdSe QD and 8.2nm for the $\mathrm{CdSe} / \mathrm{ZnS}$ QD.

The experimental setup compared the dried film fluorescence emission of each of these types of QDs on top of a control (blank glass slide) and a dried bR film (5uL of $5 \mathrm{mg} / \mathrm{ml}$ PM). Each of the QD samples was suspended in toluene at a concentration of $0.5 \mathrm{nmol} / \mathrm{ml}$. A total of $1 \mu 1$ of the $0.5 \mathrm{nmol} / \mathrm{ml}$ QD solution was applied to the desired substrate for each particular test, yielding a total deposition of $5 \times 10^{10}$ QDs. The estimated 3mm spot size of deposited QDs should create a film no more than 1 QD thick, thus allowing virtually all of the QDs to participate in FRET coupling. The resulting fluorescent emission of the dried QD films vs. substrate is shown in Figure 110.

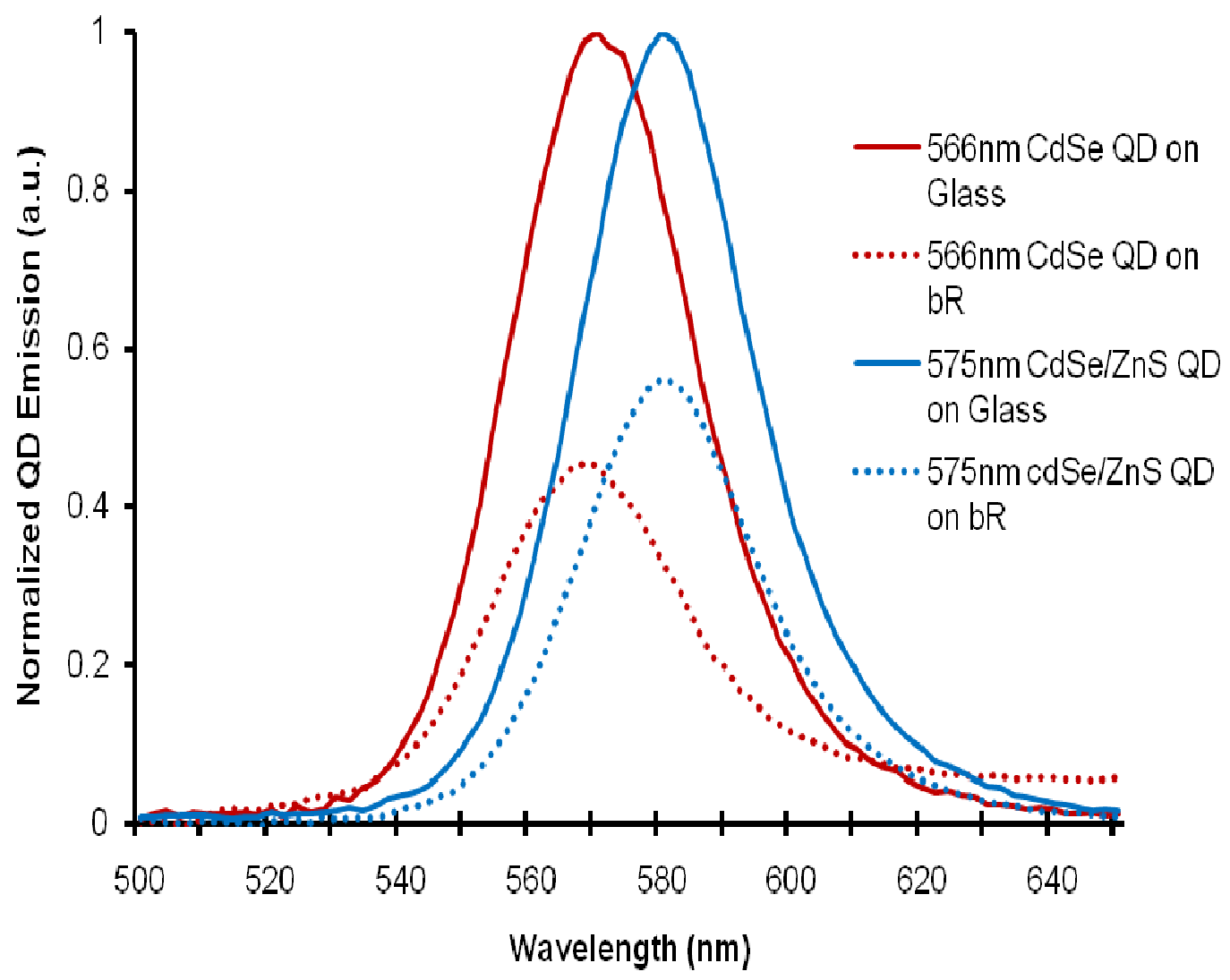

Figure 110. Reduction in CdSe and CdSe/ZnS Quantum Dot emission due to energy transfer to $b R$ in dried thin films. 
From the results it can be seen that a larger separation distance between the QD core $(\mathrm{CdSe})$ and the bR results in a decrease in FRET efficiency. Specifically the CdSe core QDs coupled to the bR with a FRET efficiency of $54.5 \%$, while the CdSe/ZnS core-shell QDs had a FRET efficiency of 43.8\%. This is explained due to the nature of FRET. The FRET process is sensitive to the donor/acceptor ( $Q D / b R)$ separation distance, thus by increasing the $\mathrm{QD} / \mathrm{bR}$ separation distance by adding polymeric shells to the QDs, less energy was transferred non-radiatively from the QD to the bR retinal. In the case of the $\mathrm{ZnS}$ shell, the FRET efficiency was reduced by $11.1 \%$. This results in the CdSe/ZnS QDs displaying more fluorescence emission than the CdSe QDs.

\subsection{QD-BR I-SAM FILM FRET}

Design of the sensing platform will require $\mathrm{bR}$ and QDs to be integrated on the nanoscale. Previous work has shown the ability to efficiently integrate these nanomaterials in I-SAM films in addition to suggesting FRET interactions. Using the aforementioned film growth techniques, bR-QD hybrid films were created, as shown in the inset of Figure 111 , and used to quantify FRET interactions. Specifically a single QD monolayer was deposited directly on top of a PDAC coated ITO electrode. The QD emission was measured with a Hitachi-F7000 fluorospectrometer and used as the control. Multiple bR monolayers were deposited on top of the QD base layer and the effect on QD emission was measured. A bar-chart representation of the QD quenching effects of each bR monolayer is displayed in Figure 112. 


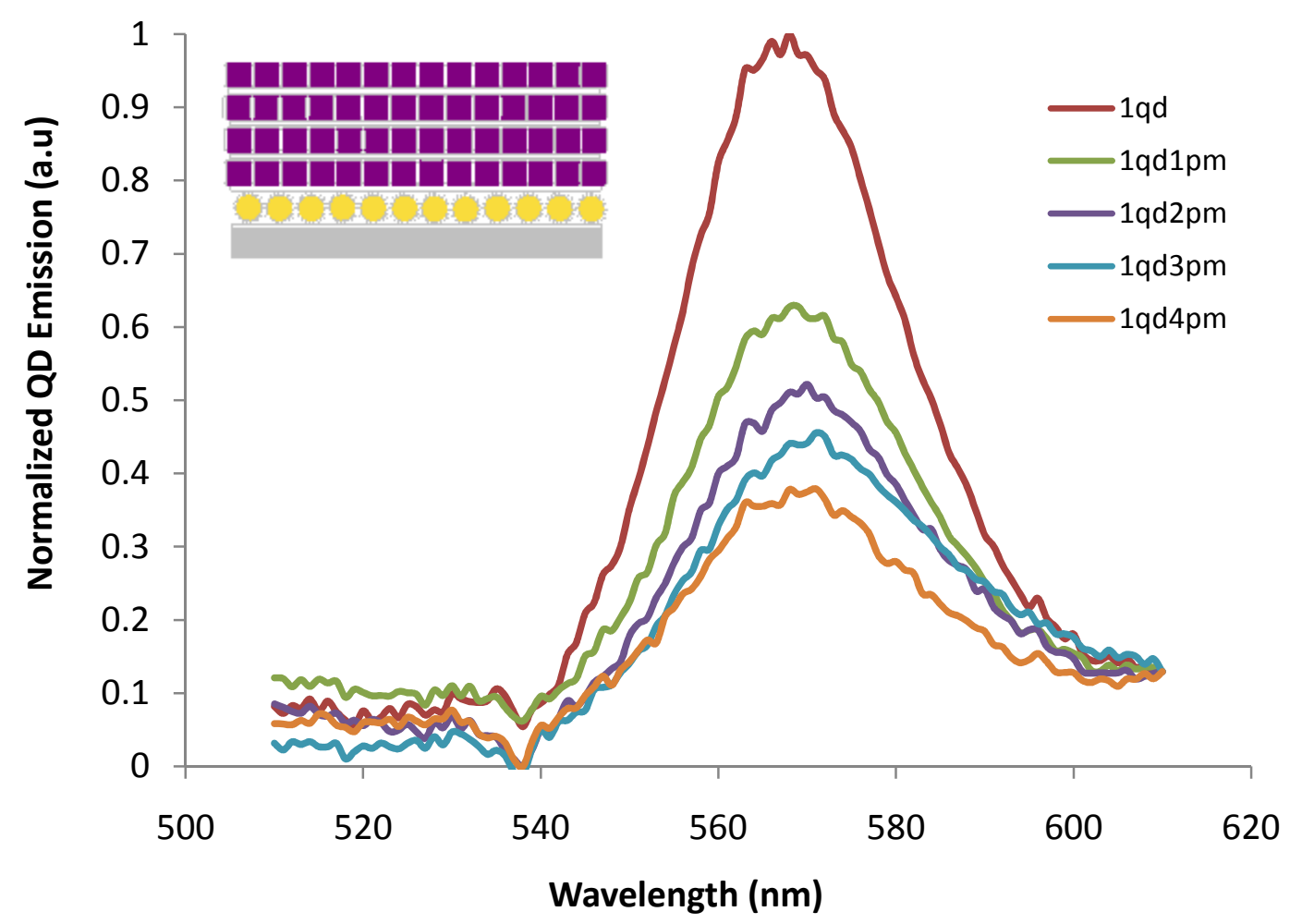

Figure 111. I-SAM bR/QD film suggesting that the first bR layer is FRET coupled to the QDs while subsequent bR layers only have photonic absorption quenching effects.

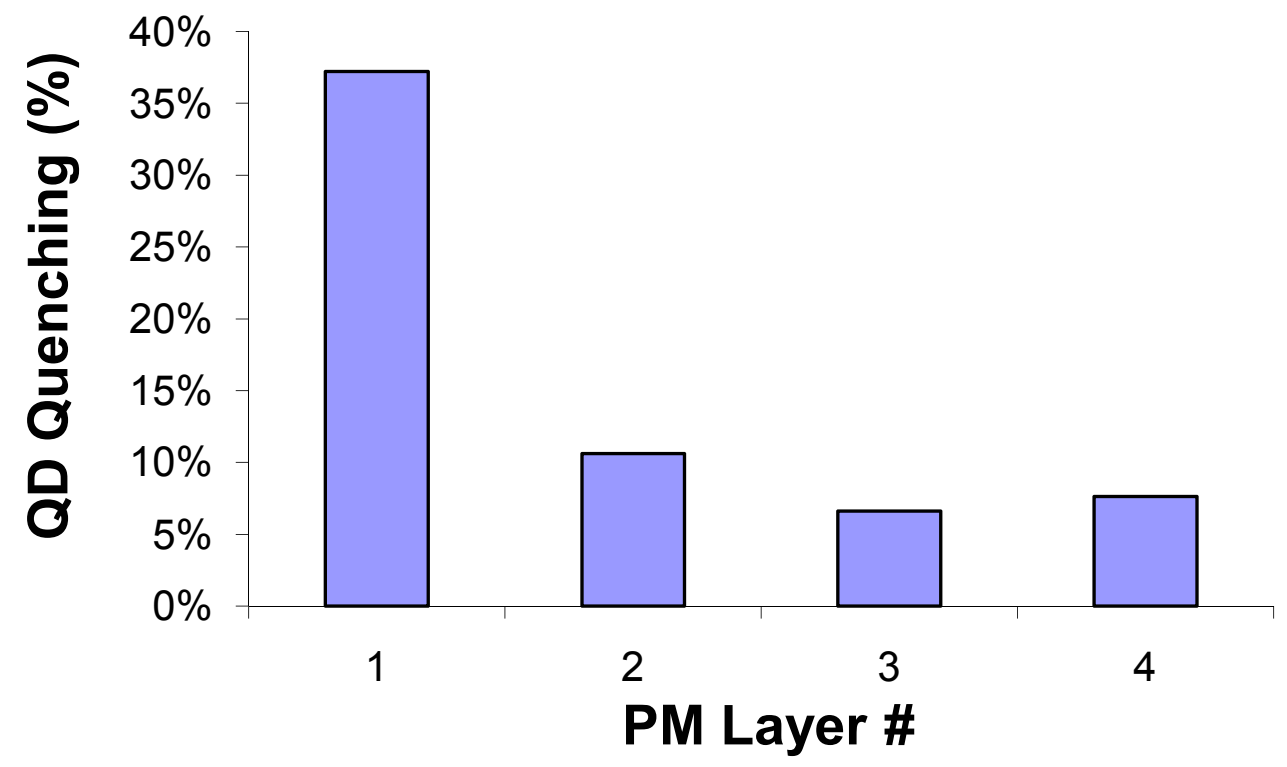

Figure 112. Quenching of QD emission in bR/QD I-SAM film, with a 37\% emission reduction from the first $b R$ layer and $<10 \%$ reduction for each subsequent $b R$ layer. 
To clarify the effects of FRET from bR optical absorption processes, layers of bR were consecutively added to a single QD monolayer as shown in Figure 111. This setup was created so each bR monolayer should be deposited at a subsequently further distance from the base QD monolayer. As shown in Figure 112, the first bR monolayer induced a $39 \%$ reduction in QD emission. Since FRET is primarily observed when the acceptor (bR)/ donor (QD) pair are within sub-10nm proximity, and thus resonance energy transfer is the probable cause for the quenching seen by the initial layer of purple membrane. As the PM is $5.5 \mathrm{~nm}$ thick, each PDAC layer is approximately $0.5 \mathrm{~nm}$ thick, the QD ZnS shell is $1 \mathrm{~nm}$, and the retinal is located in the middle of the $b R$ molecule, it was estimated that the retinal of the $2^{\text {nd }}$ deposited bR layer is $9.5 \mathrm{~nm}$ from the QD core. This distance is close to the limits of FRET coupling, thus should contribute little to QD quenching via FRET interactions. Results support this claim, as the $2^{\text {nd }} \mathrm{bR}$ monolayer only reduced the QD emission by $10 \%$, which is about $75 \%$ less than the QD quenching effects of the $1^{\text {st }}$ bR monolayer. Subsequent layers provide a relatively consistent 7\% reduction in QD emission. Therefore it is likely that the $2^{\text {nd }}$ layer, at its $9.5 \mathrm{~nm}$ bR retinal-QD separation distance, could contribute partially via FRET but primarly contributes to the QD quenching via photonic absorption. The lowered quenching effects and consistency in emission reduction from the final two bR monolayers suggests that their primary contribution to the QD quenching is due to photonic absorption. 


\section{Chapter 7. MBP Sensor Proof of Concept}

To modulate the photovoltaic output of this system, fluorescence resonance energy transfer (FRET) is being investigated. The physical dimensions of the MBP molecule is $3 \mathrm{~nm} \times 4 \mathrm{~nm} \times 6.5 \mathrm{~nm}$ [128]. The protein consists of two globular domains that has a $1.8 \mathrm{~nm} \times 0.9 \mathrm{~nm} \times 1.8 \mathrm{~nm}$ groove in the middle; which serves as the maltose binding location [129]. The maltose binding mechanism is achieved through hydrogen bond and aromatic residue stacking [128-130].

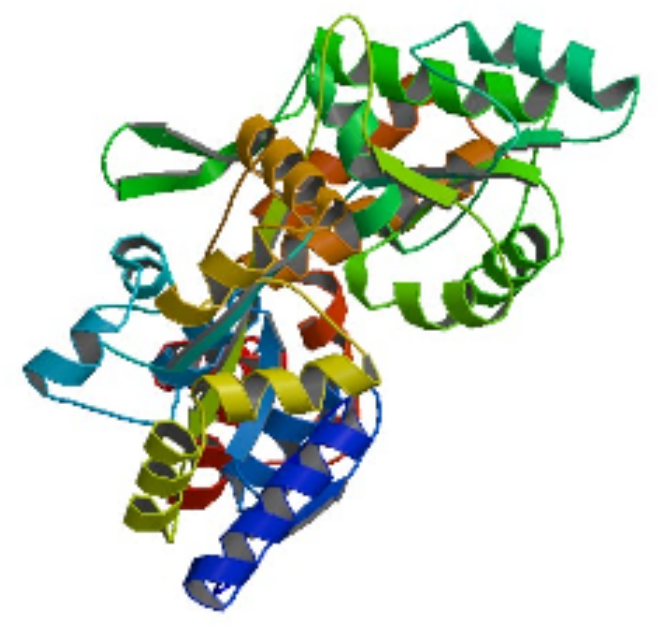

Figure 113. Ribbon diagram of Maltose Binding Protein.

The binding pocket of MBP has the ability to not only bind to maltose, but also to molecules such as $\beta$-cyclodextrin ( $\beta$ - $C D)$, although the binding kinetics are different [131]. When binding to $\beta$-CD only a few hydrogen bonds are formed and the MBP molecule remains in its open configuration. Thus the binding affinity of MBP to B-CD is only about half what it is for maltose, with MBP binding affinities of $1.8 \mu \mathrm{M}$ and $3.5 \mu \mathrm{M}$ respectively [124].

Previous work has shown that additional molecules can be attached to the $\beta$-CD and delivered into close proximity with the MBP upon B-CD binding and is more thoroughly 
discussed in the introduction section. Applications of the multiple binding affinities of MBP have focused on the creation of MBP-QD maltose-sensors, where the $\beta-C D$ is bound to a quencher molecule that ultimately FRET couples with the QD upon MBP binding. The quencher molecules can be fluorescent tags, such as Cy 3.5, or a dark quencher molecule, such as QSY9. Ultimately the introduction of maltose into the system will replace $\beta-C D$ in the MBP binding site and eliminate the FRET coupling relationship. The sensing is achieved by monitoring the return of QD emission to its original state. To date these types have sensors have only been demonstrated with the MBP-QD pair in an aqueous system. This study will explore the capability of applying this sensor concept in dried film form, ultimately using it variable QD photon source for bR activation. A schematic of the proposed sensing scheme is displayed in Figure 114.

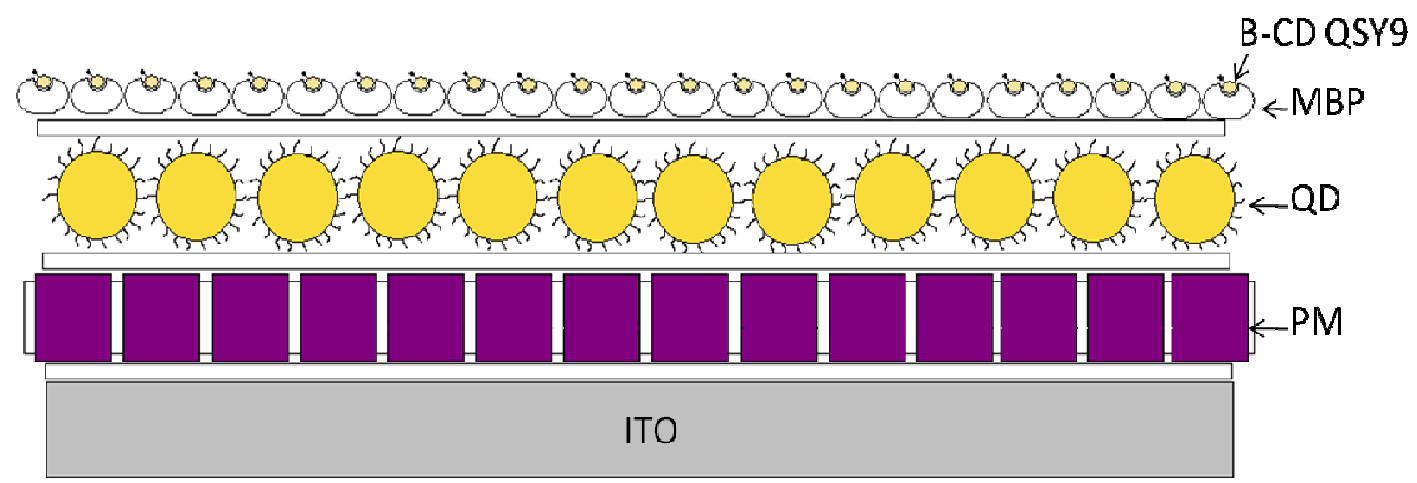

Figure 114. Proposed maltose sensor schematic.

This system proposes to utilize the ability for the dark quencher $\beta$-CD-QSY9 to FRET couple with the QD monolayer film to quench the QD emission. The bR photovoltaic output is directly related to the amount of photons being absorbed by the retinal, thus as of QD photons are decreased the bR output will likewise be depressed. 


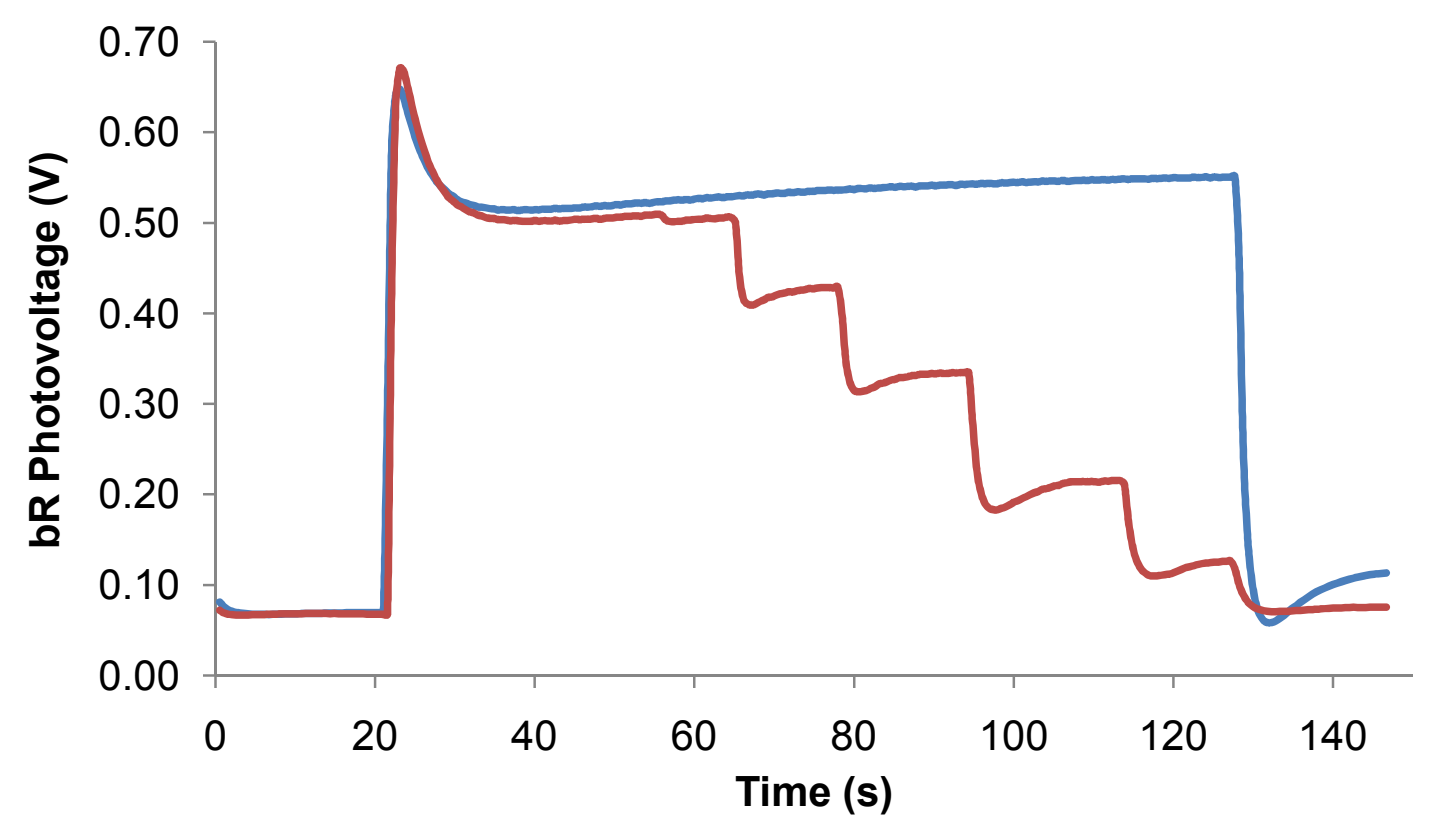

Figure 115. Effect of incident light intensity variation on bR photovoltage.

\subsection{Synthesis and PuRIfication of Maltose Binding Protein}

The maltose binding protein (MBP) used in this work was produced by the genetically engineered strain of E. coli JM109 [132-134]. The frozen stock of E. coli JM109 was suspended in $3 \mathrm{ml}$ of lysogeny broth (LB) or terrific broth (TB) at $37^{\circ} \mathrm{C}$. A total of $0.1 \mathrm{ml}$ of these cells were used as inoculant in $4 \mathrm{ml}$ of M9 minimal medium containing maltose as the sole source of carbon, the composition of $500 \mathrm{~mL}$ of M9 media is displayed in. The E. coli JM109 cells were allowed to reach an $\mathrm{OD}_{600}$ of 0.50 and then these cells are used to inoculate 1 litre of the same medium and the cells grown to logarithmic phase ( $\sim 8$ to $\left.1.0 \mathrm{OD}_{600}\right)$.

Once grown the cells are collected by centrifugation at 5,000 $\mathrm{g}$ for 30 minutes at $4^{\circ} \mathrm{C}$. The pelleted cells are resuspended in binding buffer of $25 \mathrm{mM}$ MOPS. $0.2 \mathrm{M}$ sodium chloride, and $1 \mathrm{mM}$ EDTA adjusted to $\mathrm{pH} 7.4$ using $0.5 \mathrm{M} \mathrm{NaOH}$. Approximately $15 \mathrm{ml}$ of 
buffer per gram of wet cell weight was used. The total volume of buffer should not exceed $50 \mathrm{ml}$.

Table 9. M9 media preparation ingredients.

\begin{tabular}{|c|c|}
\hline Chemical & Amount (g) \\
\hline $\mathrm{Na}_{2} \mathrm{HPO}_{4}$ & 30 \\
\hline $\mathrm{KH}_{2} \mathrm{PO}_{4}$ & 15 \\
\hline $\mathrm{NaCl}$ & 2.5 \\
\hline $\mathrm{MgSO}_{4} \cdot 7 \mathrm{H}_{2} \mathrm{O}$ & 1.25 \\
\hline $\mathrm{NH}_{4} \mathrm{Cl}$ & 5 \\
\hline Maltose (autoclaved separately) & 10 \\
\hline
\end{tabular}

After thoroughly resuspending the cells, place them in the freezer at $-20^{\circ} \mathrm{C}$. Cell lysis is enhanced during the slow freezing when the cells aren't frozen as pellets. Thaw the cells at room temperature in a beaker containing room temperature water. When the cells lyse, the suspension will become thick and viscous from the release of nucleic acids. Reduce the viscosity by sonicating the suspensions for 2 minutes $(30 \%$ power, $50 \%$ pulse regimen) while the cells are in an ice bath. DNase I can be added prior to sonication to help reduce viscosity at $1 \mathrm{Unit} / \mathrm{ml}$. Large cell debris is pelleted through centrifugation for 30 minutes at $10,000 \mathrm{~g}$. After centrifugation the supernatant is decanted into a fresh container and the pellet is discarded. If the pellet is soft and a portion of the pellet decants into the supernatant pool, centrifuge the supernatant again to remove the residual cell debris.

The final supernatant is filtered through a $0.45 \mu \mathrm{m}$ filter to remove any remaining whole cells. This is a precaution so that residual whole cells won't interfere with the affinity the following chromatography step. The filter extract is diluted with 4 volumes of binding buffer (25mM MOPS, pH 7.4, with 0.2M sodium chloride and 1mM EDTA). Protein 
concentration of the filtered extract is determined with the BCA reagent, with BSA as standard. Final protein concentration should be about $2-6 \mathrm{mg} / \mathrm{ml}$.

An amylose resin with a binding capacity of $7 \mathrm{mg}$ of MBP per $\mathrm{ml}$ of suspended resin is used to purify the MBP. An appropriate amount of resin is equilibrated in binding buffer in a small column. The amount of resin necessary depends upon the amount of MBP in the extract. The amount of MBP in the extract should not be more than the maximum capacity of the resin in the column; otherwise, MBP may pass through the column. In theory, approximately 5 to $10 \%$ of the total protein would be MBP, unless an overproduced plasmid is used and up to $30 \%$ of total protein can be achieved.

The crude extract is loaded onto the column at a flow rate of approximately 5-10 column volumes per hour. Exceeding this load rate will result in some of the MBP not binding to the resin. The extract should be loaded immediately after preparation and the loading time should not be greater than 6 hours. After all of the extract is loaded, the column is washed with fresh binding buffer. The flow rate during the washing step can be increased to 10-20 column volumes per hour. The column should be extensively washed until the $\mathrm{A}_{280}$ of the material eluting from the column is $<0.05$. This means that all unbound protein has been washed off the column.

The final MBP product is eluted from the column using 10 column volumes of elution buffer $(25 \mathrm{mM}$ MOPS, $\mathrm{pH} 7.4$, with $0.2 \mathrm{M}$ sodium chloride, $1 \mathrm{mM}$ EDTA, and $10 \mathrm{mM}$ maltose). The MBP should begin to elute after the first column void volume has passed through the column, but elution could be slower. At least 2 column volumes worth of fractions should be taken. The fractions are be monitored by $\mathrm{A}_{280}$ and those with absorbance above background are be analyzed by SDS-PAGE. The filter MBP samples are concentrated useing Millipore-Centricon centrifuge filter devices. 


\subsection{SYNTHESIS AND PURIFICATION OF DARK QUENCHER}

The $\beta$-Cyclodextrin-QSY-9 adduct used in these studies was prepared according to previously established procedures $[41,124]$. This procedure involved the linking of 6monodeoxy-6-monoamino- $B$-cyclodextrin ( $\beta$-CD) with NHS-QSY9. Here, as shown in , the $\beta$-CD was used to bind to the MBP and physically carry the QSY9 molecule to perform the energy quenching.

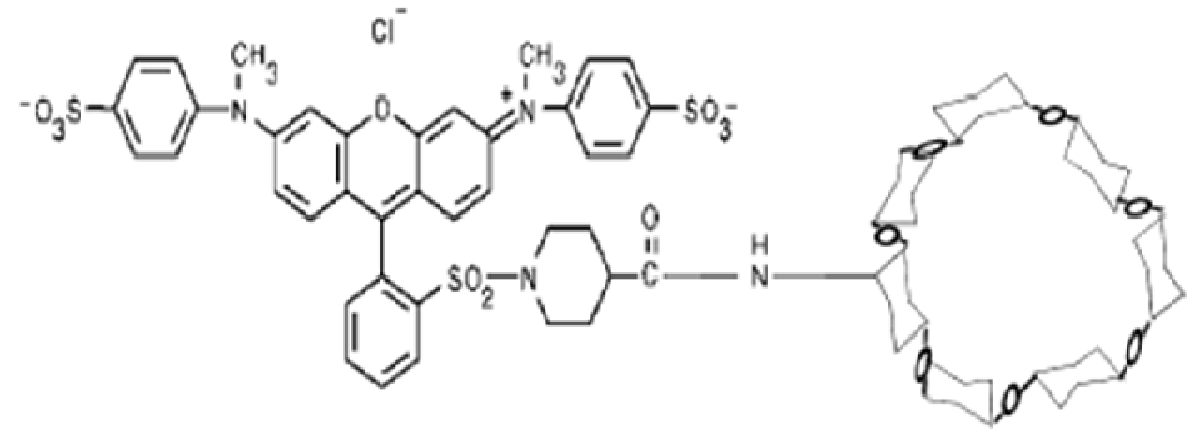

Figure 116. Diagram of $\beta$-CD-QSY9 conjugate molecule for binding with MBP [41]. Reprinted by permission from Macmillan Publishers Ltd: Nature Materials, Medintz, I.L., et al., Self-assembled nanoscale biosensors based on quantum dot FRET donors, 2: p. 630-638, copyright (2003) and permissions can be found in Appendix A.6.

In summary, an 8-fold molar excess of 6-monodeoxy-6-monoamino- $B$-cyclodextrinwas reacted with monofunctional NHS-QSY9 for 2.5 hours in $0.136 \mathrm{M}$ sodium borate buffer, adjusted to $\mathrm{pH} 8.5$ via $5 \mathrm{M} \mathrm{NaOH}$. All reactions were performed in a $15 \mathrm{~mL}$ thick-walled corex tube with $1 \mathrm{~mL}$ total of sodium borate buffer. The relative molecular weights and molar comparisons are displayed in Table 10. The $\beta$-CD-dye adduct and excess monoamino $B$-CD was then precipitated by adding at least three volumes of cold ethanol (3-6mL) followed by holding the preparation at $-20^{\circ} \mathrm{C}$ for 1 hour. The precipitate, collected by centrifugation, was washed four times with absolute ethanol and finally resuspended in $0.5 \mathrm{~mL}$ ethanol. The final material was collected again by centrifugation at 10,000 RPM for 10 minutes $\left(4^{\circ} \mathrm{C}\right)$, and dried in a vacuum centrifuge. 
Table 10. $\beta$-CD and QSY-9 molar comparisons for conjugate synthesis.

\begin{tabular}{|c|c|c|c|l|}
\cline { 2 - 5 } \multicolumn{1}{c|}{} & MW $(\mathrm{g} / \mathrm{mol})$ & Molar Excess & Amt. Used $(\mathrm{mg})$ & Moles used \\
\hline$\beta-C D$ & 1170.46 & 8 & 100 & $8.54365 \mathrm{E}-05$ \\
\hline QSY-9 & 951.43 & - & 10.16085556 & $1.06796 \mathrm{E}-05$ \\
\hline
\end{tabular}

Dried pellets were solubilized in 0.1-0.2mL HE buffer (10mM HEPES, 1mM EDTA buffer, $\mathrm{pH}$ 7.0) and the product was separated from unreacted $B-\mathrm{CD}$ and underivatized dye on $12 \%$ acrylamide $1 \times$ TBE gels. The product band was excised and the $B$-CD-dye adduct eluted by passive diffusion overnight into HE buffer. This step can be repeated several times to achieve full diffusion of product.

The $\beta$-CD-dye adduct was then concentrated and desalted using an oligonucleotide purification cartridge (OPC, Applied Biosystems, Foster Cißty, CA). The dilute B-CDdye adduct in HE buffer was passed over the OPC several times to promote full binding to the resin. The bound $\beta$-CD-dye adduct was washed with $35 \mathrm{~mL}$ of $25 \mathrm{mM}$ triethylamine ammonium acetate (TEAA) buffer $(\mathrm{pH} 7)$ and then eluted from the OPC with $1 \mathrm{~mL} 50 \%$ acetonitrile to remove the final product. The eluted product solution was aliquoted into $1.5 \mathrm{~mL}$ microfuge tubes, dried under vacuum, and stored at $-20^{\circ} \mathrm{C}$.

Using the Nanodrop the amounts of dark quencher adduct was quantified using the known extinction coefficient of $86,000 \mathrm{M}^{-1} \mathrm{~cm}^{-1}$ for QSY9 at $562 \mathrm{~nm}$. The nanodrop reading from this work is shown in Figure 117. The final product yield was approximately $22 \mu \mathrm{g}$ of $\beta$-CD QSY9 dark quencher product. 


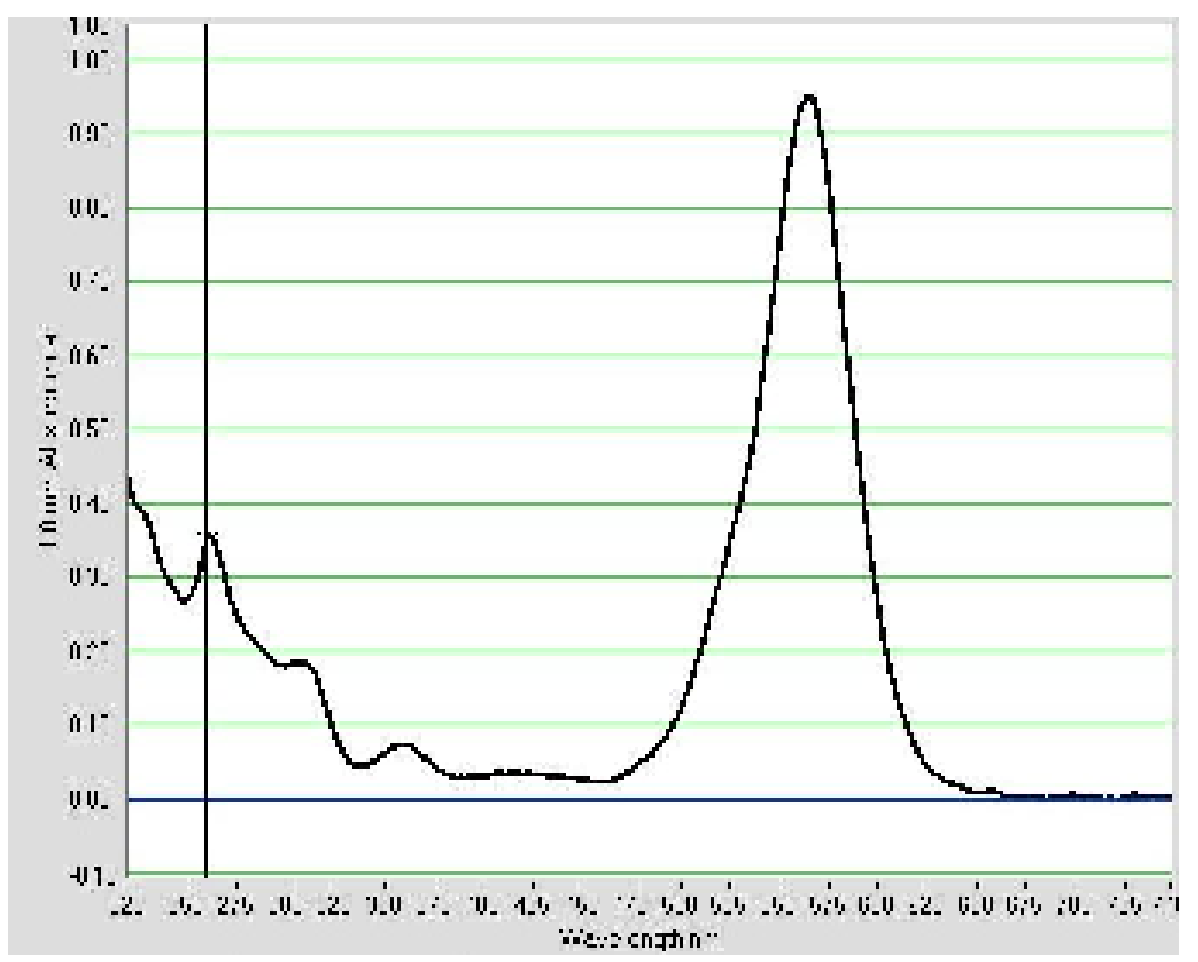

Figure 117. Nanodrop absorbance spectra of as-synthesized $\beta-C D-Q S Y-9$ dark quencher.

\subsection{QD-MBP HYBRID I-SAM FILMS}

The dark quencher sensing scheme developed by Medintz et al. [41, 124] was developed using QDs and mixtures in an aqueous suspension. Thus the proposed proof of concept sensor will initially require the implementation of this sensing scheme into thin film form. Specifically it will be required to deposit a MBP monolayer in nano-scale proximity to the QD monolayer. This deposition must put the nanomaterials into close enough proximity to allow MBP bound B-CD-QSY9 dark quencher molecule to be in FRET coupling proximity of the QD. Theoretically, since both the MBP and QD will be applied in I-SAM films with their negative charge, the positive polycation adhesion layer, PDAC, will only induce a $0.5 \mathrm{~nm}$ separation distance between the QD and MBP [82]. The orientation of MBP after adhesion via the I-SAM technique is unknown, thus an exact knowledge of the binding pocket location is unknown. With a $1.5 \mathrm{~nm}$ thick QD shell and MBP dimensions of $3 \times 4 \times 6.5 \mathrm{~nm}$ [135], the binding pocket location can be 
estimated to be $2 \mathrm{~nm}-8.5 \mathrm{~nm}$ from the QD core. Since FRET is typically observed between acceptor/donor molecules within sub-10nm separation distance, the QD-MBP integrated monolayer films should bring the dark quencher within FRET proximity of the QD.

Maltose binding protein contains carboxyl groups, which can be deprotonated at elevated $\mathrm{pH}$ levels and give the protein a net negative charge. The isoelectric point of MBP produced from E. coli occurs approximately at $\mathrm{pH} 4.8$ [136], which is similar to that of bR. For I-SAM deposition MBP was suspended at approximately $0.5 \mathrm{mg} / \mathrm{ml}$ in $10 \mathrm{mM}$ borate buffer, $10 \mathrm{mM} \mathrm{NaCl} \mathrm{pH} 8.5$.

To test the efficiency of the QD-MBP sensing scheme in dried films, a single monolayer QD substrate was deposited on ITO via previously described methods. A positively charged PDAC adhesion layer was deposited on top of the QD monolayer prior to the addition of MBP. The positively charged slide is then dipped in the MBP solution for 5 minutes following by a 2 minute rinse step. A custom fixture was created to hold the functionalized test slide in a cuvette at approximately $45^{\circ}$ relative to the Hitachi F-7000 illumination source. The optics leading to the photomultiplier tube is at $90^{\circ}$ to the illumination optics, thus the slide was at an adjusted angle for optimal activation and photonic detection. The prepared slide was placed in the fixture and $1 \mathrm{~mL}$ of $100 \mathrm{mM}$ $\mathrm{NaCl}, 10 \mathrm{mM} \mathrm{KPO}_{4} \mathrm{pH} 7$ was added to the cuvette. After measurement of the initial QD emission from the QD-MBP substrate, the slide was removed and dipped in a $0.11 \mu \mathrm{g} / \mathrm{ml}$ solution of $\beta$-CD-QSY9 dark quencher solution for 1 minute. The slide was then thoroughly rinsed for 2 minutes in ddI water to remove any unbound dark quencher and the QD emission was again measured. Varying concentrations of then maltose was then added to the cuvette and allowed to sit for 5 minutes. The QD emission was measured at each incremental maltose addition. The process for sensor testing is given in Figure 118 and the resulting QD emission at each stage is shown in Figure 119. 


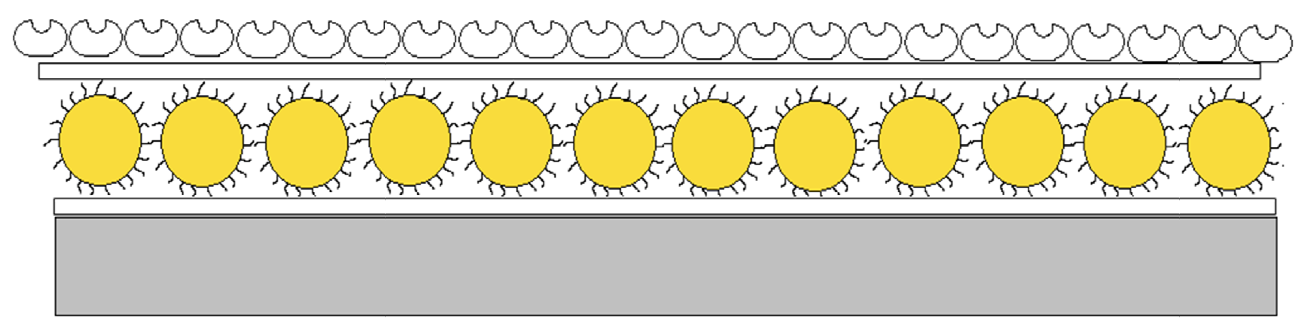

- B-CD-QSY9 binds to Maltose Binding Protein (MBP)

- QSY9 FRET couples to QD, no energy re-emission

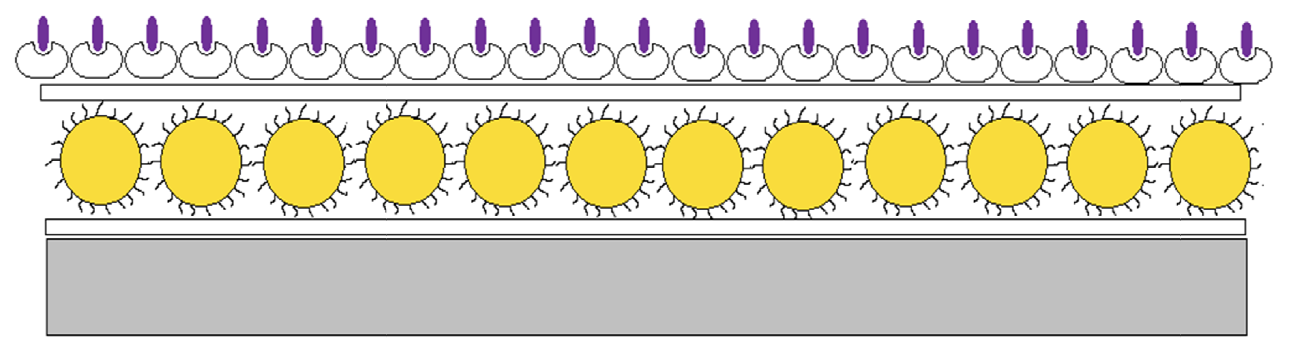

- Maltose has higher binding affinity to MBP

- Competively displaces B-CD-QSY9

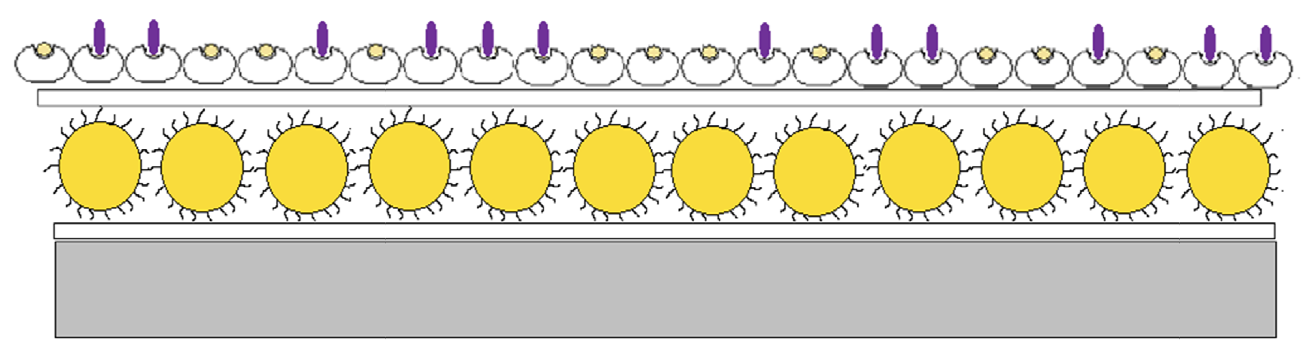

- Displacement of B-CD-QSY9 restores QD emission

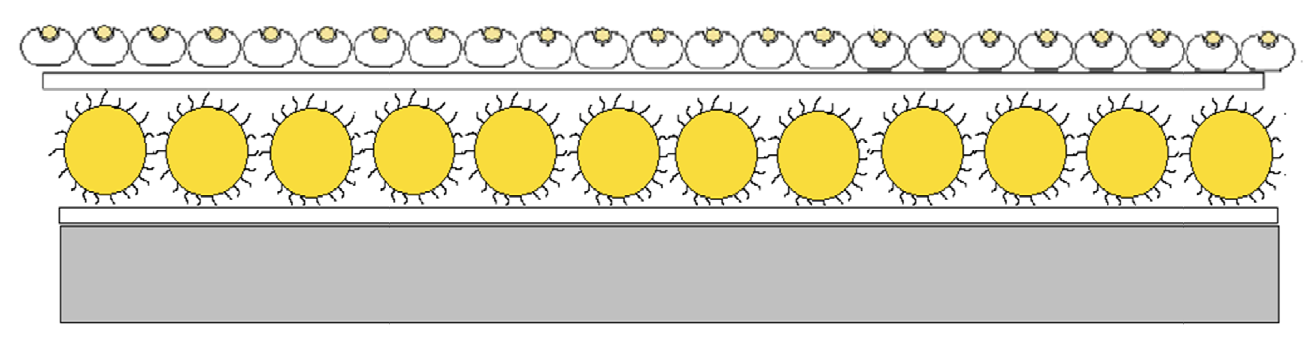

Figure 118. Schematic of QD-MBP thin film biosensor for maltose sensing. 


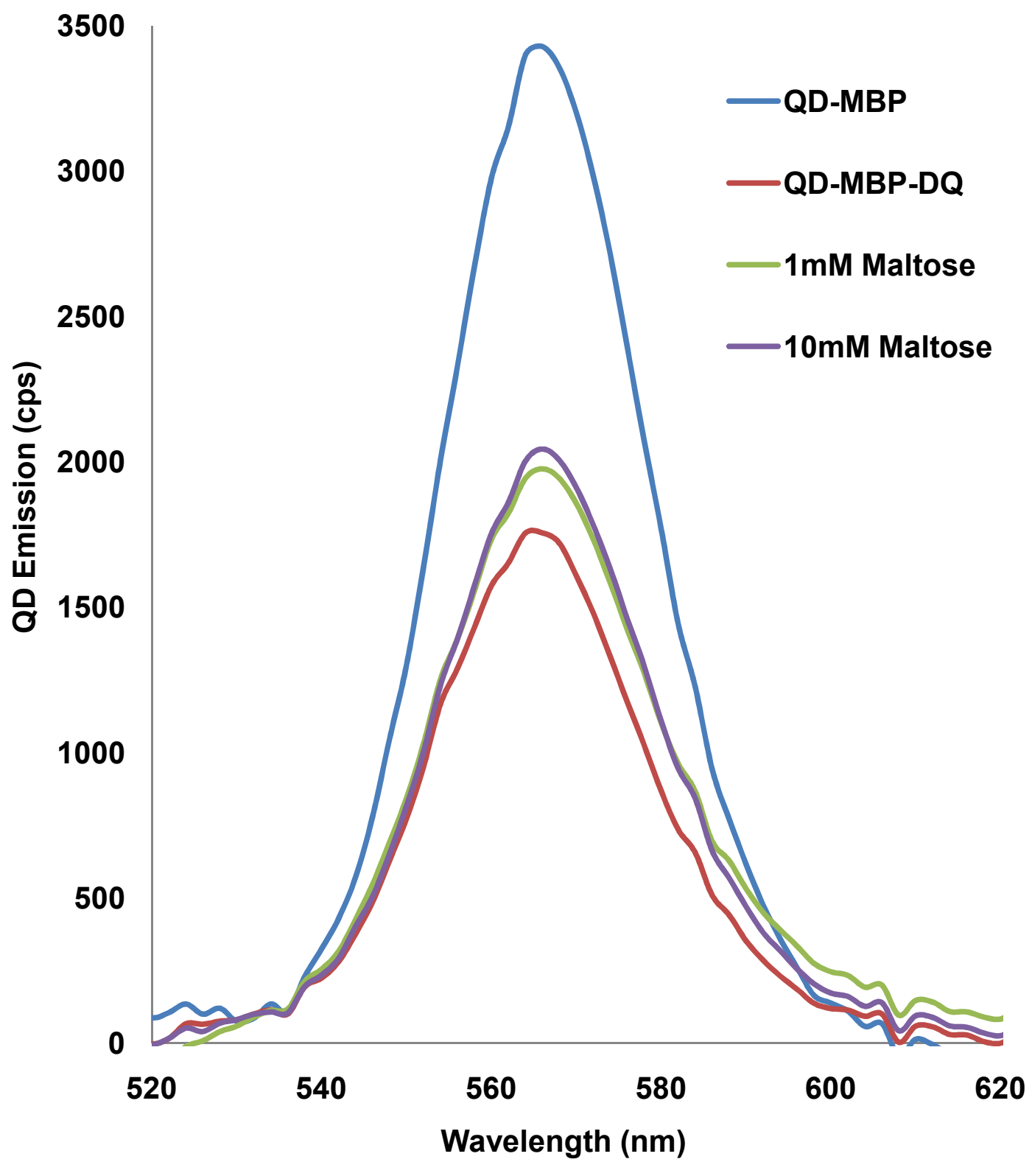

Figure 119. QD emission during maltose detection with a QD-MBP I-SAM film.

It can be seen in Figure 119 that the binding of $\beta-\mathrm{CD}-\mathrm{QSY} 9$ dark quencher molecules to the QD-MBP hybrid substrate reduces the QD emission by nearly 50\%. This is likely due to a combination of QD photon absorption and direct energy coupling of the dark quencher molecule. Subsequent addition of maltose resulted in displacement of the dark 
quencher and partial restoration of the QD emission. Specifically it was found that incubation of the slide in a $1 \mathrm{mM}$ maltose solution for 5 minutes resulted in a QD emission increase of $14 \%$ from the measured dark quencher state. Subsequent incubation of the test slide in a $10 \mathrm{mM}$ solution resulted in a total QD emission increase of nearly $17 \%$ from the dark quencher state.

This work shows the viability of a QD-MBP hybrid I-SAM film to perform maltose sensing via the dark quencher method. It is noted, however, that the sensitivity of this film seems much lower than that reported by the aqueous suspension version, which reported sensing down to the $1 \mu \mathrm{M}$ maltose range. It is possible that integrating a solution mixing scheme into this setup will enhance maltose displacement of the dark quencher molecule and improve sensitivity.

\subsection{BR-QD BIO-NANOSENSING SUBSTRATE}

With the ability to modulate the QD energy level via the QD-MBP hybrid I-SAM sensing substrate described above achieved, the next step was to integrate the bR transduction layer. Using the I-SAM deposition techniques previously described, the deposition of $\mathrm{bR}, \mathrm{QD}$, and MBP integrated monolayers was achieved to the desired film structure. A custom cuvette was created to hold the functionalized electrode and a $0.5 \mathrm{~mm}$ platinum wire counter electrode at a separation distance of $1 \mathrm{~cm}$. The entire setup was fixed in a faraday cage and connected to an $11 \mathrm{x}$ high impedance operational amplifier. The signal output was measured on an oscilloscope (Agilent 54620A) and recorded for later analysis. An outline of the testing procedure is shown in Figure 120. 


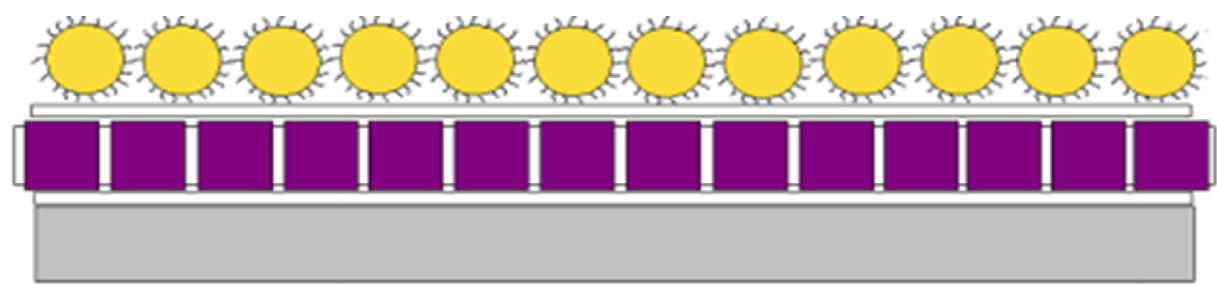

-Add Maltose Binding Protein (MBP) monolayer
as receptor molecule.

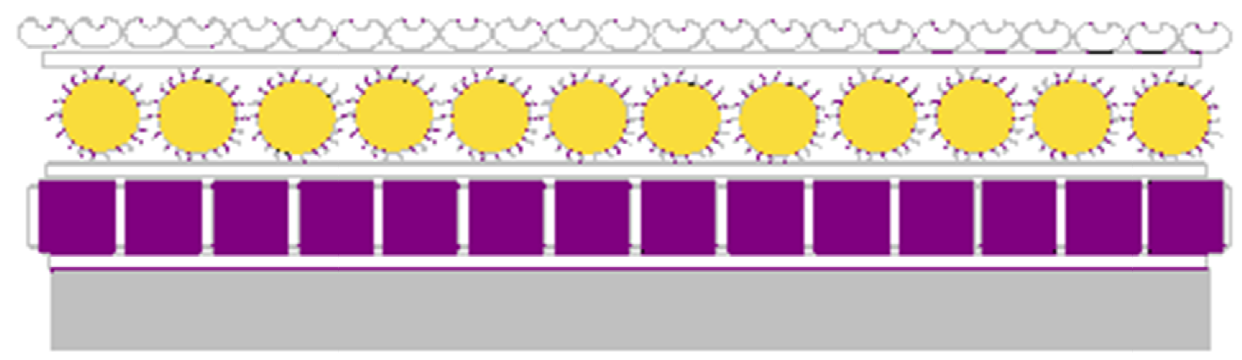

- B-CD-QSY9 binds to MBP and FRET couples with QD.

- Reduces QD energy to bR, lowers bR output.

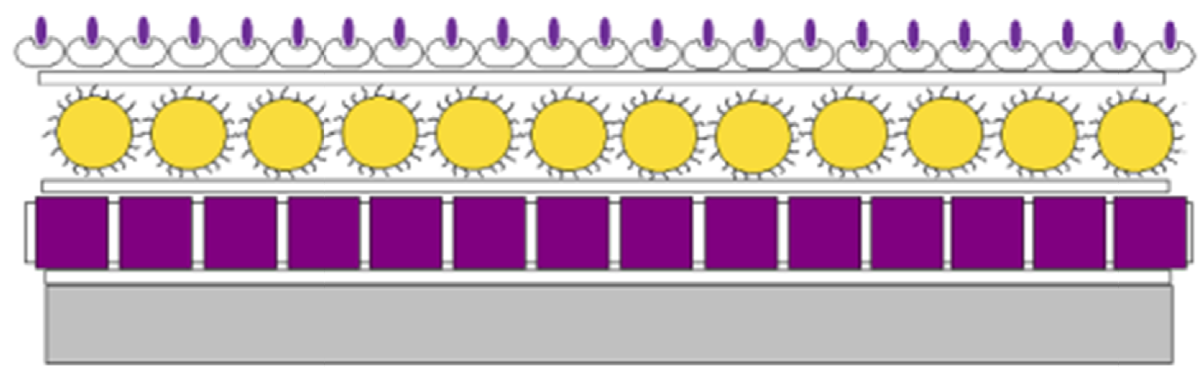

- Displacement of B-CD-QSY9 restores QD energy.
QQD-bR energy transfer restored, bR output increases.

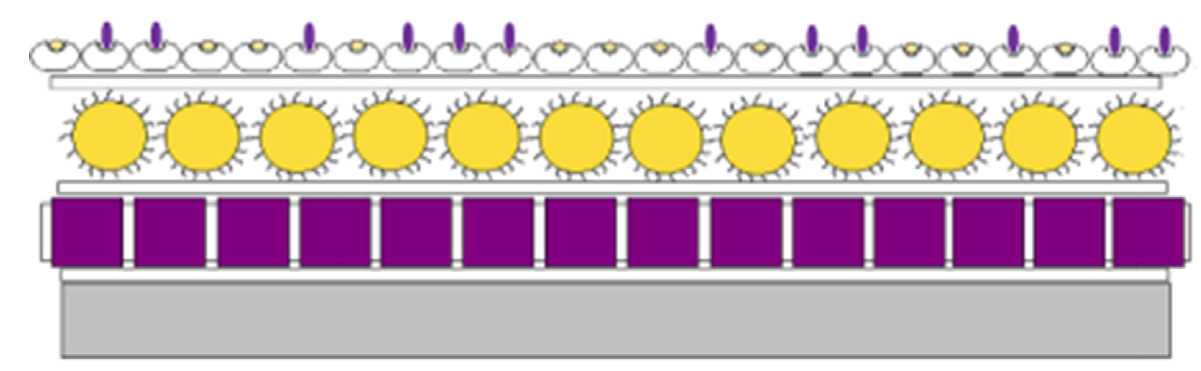

Figure 120. bR-QD hybrid substrate functionalized with a MBP receptor for the detection of maltose. 
For this study a single bR monolayer was deposited on the ITO electrode, followed by a monolayer of $565 \mathrm{~nm}$ emission carboxyl QDs. The prepared slide was placed in the fixture and $0.4 \mathrm{~mL}$ of $50 \mathrm{mM} \mathrm{KCl}, 5 \mathrm{mM}$ sodium azide deionized water was added to the cuvette. The bR photovoltaic output of the initial output is shown in Figure 121. The slide is then removed to add the MBP receptor monolayer and the bR output was again measured, as shown in Figure 122. With the MBP receptor molecule in place the substrate was ready for dark quencher binding. The slide was removed and dipped in a $0.11 \mu \mathrm{g} / \mathrm{ml}$ solution of $\beta$-CD-QSY9 dark quencher solution for 1 minute. The slide was then thoroughly rinsed for 2 minutes in ddI water to remove any unbound dark quencher and the bR photovoltaic activity was measured, resulting in the output shown in Figure 123. At this point the slide was no longer removed from the measurement cuvette. A total of $200 \mu \mathrm{L}$ of $10 \mathrm{mM}$ maltose was added to the cuvette, resulting in an approximate $3 \mathrm{mM}$ maltose concentration, and allowed to sit for 5 minutes. The resulting bR output modulation resulting from maltose displacement of the dark quencher molecule is shown in Figure 124. An overall comparison of peak bR photovoltage outputs at each sensing stage is shown in Table 11.

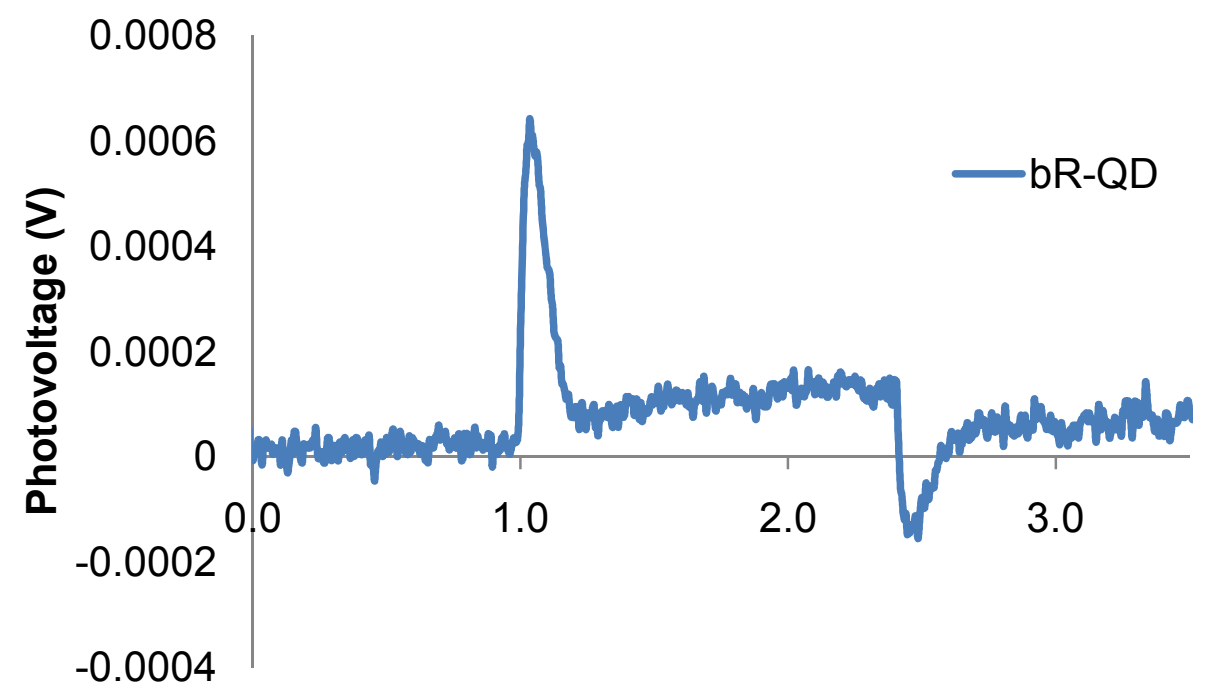

Time (s)

Figure 121. Photovoltaic response of 1 monolayer bR and 1 monolayer QD substrate. 


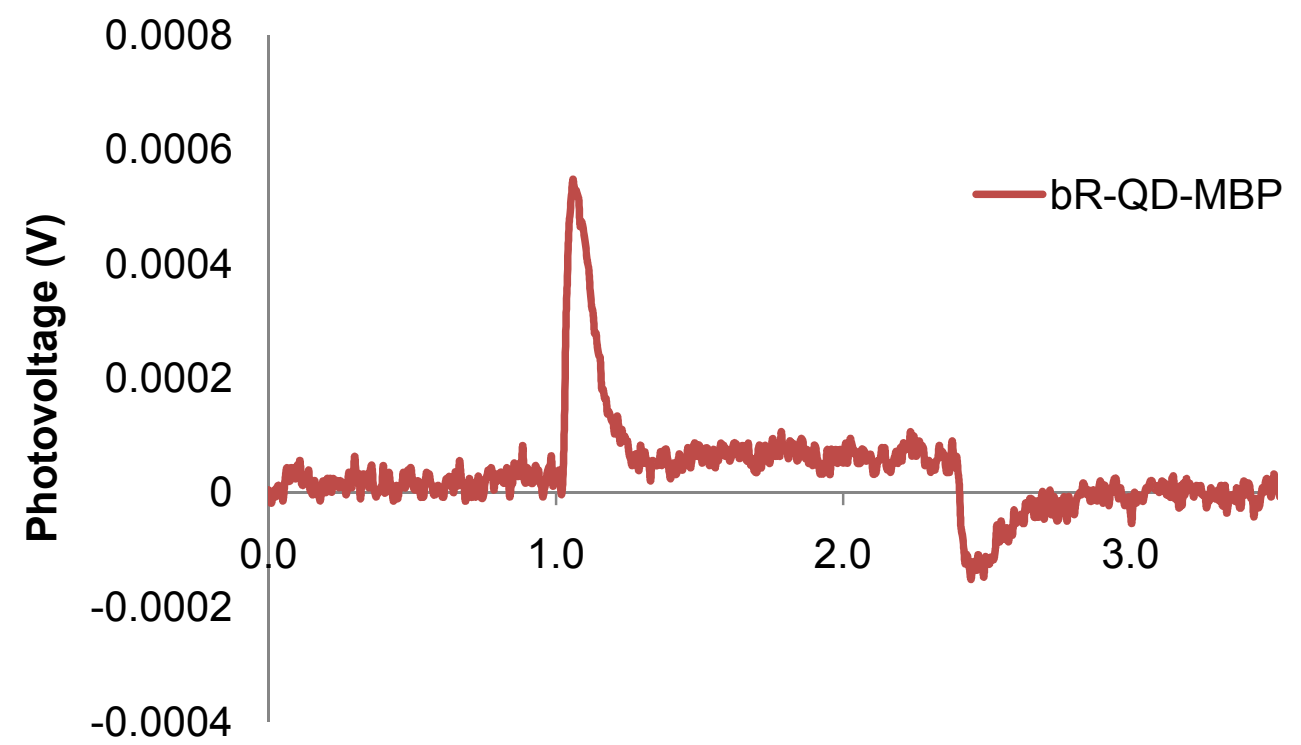

Time (s)

Figure 122. Photovoltaic response of bR-QD substrate with 1 monolayer MBP deposited on top.

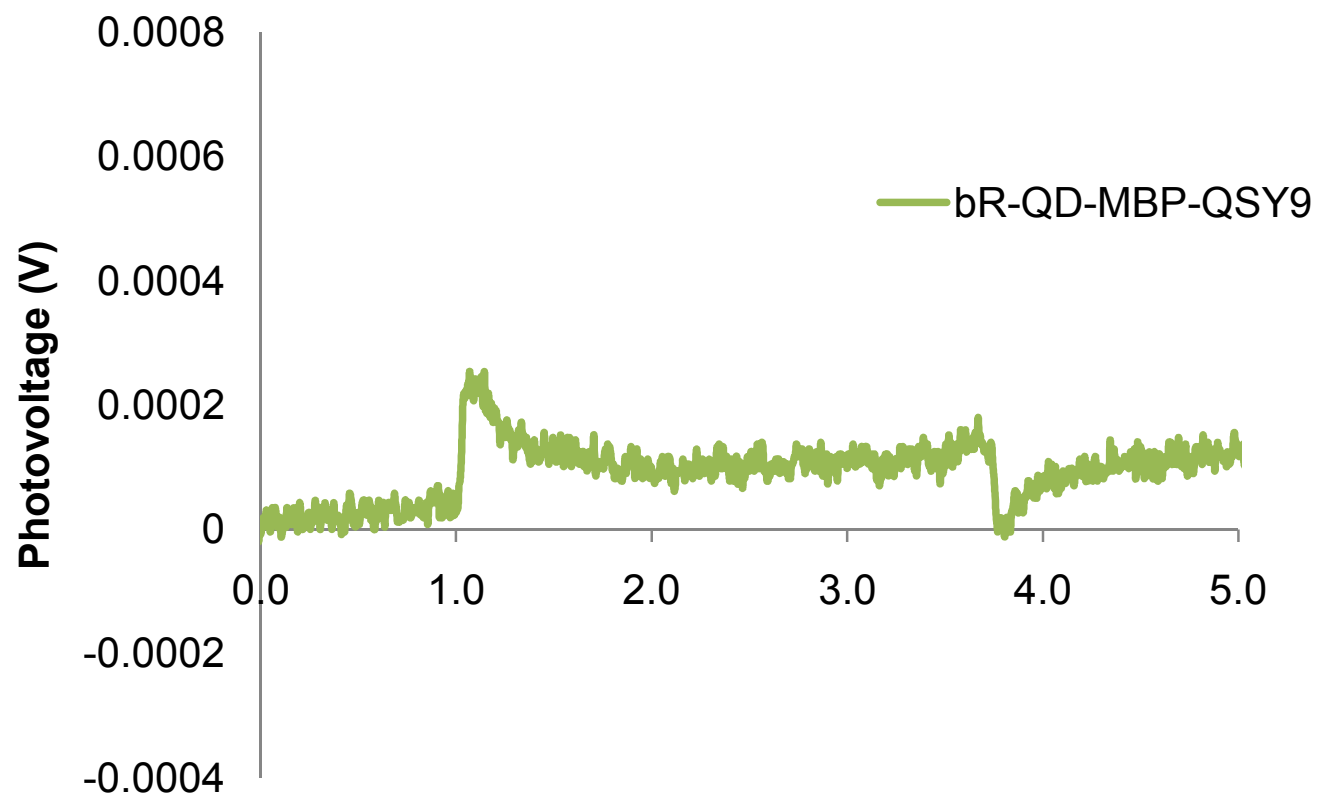

Time (s)

Figure 123. Photovoltaic response of bR-QD substrate with dark quencher bound to MBP. 


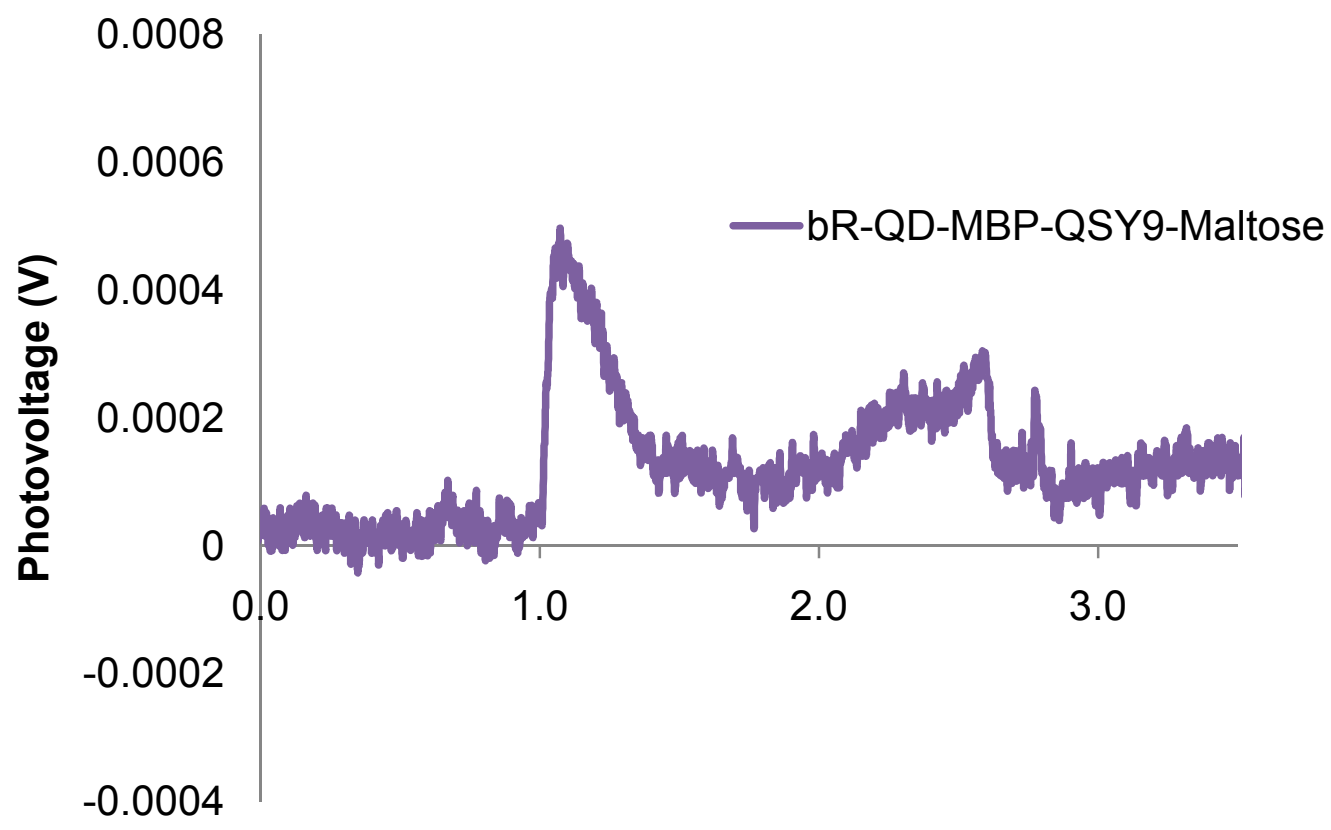

Time (s)

Figure 124. Photovoltaic response of $b R-Q D$ substrate of $3 \mathrm{mM}$ maltose addition to displace dark quencher.

Table 11. Peak photovoltaic response of bR-QD substrate at each sensing step.

\begin{tabular}{|l|c|}
\hline Substrate & Peak bR Photovoltage (mV) \\
\hline bR-QD & 0.656 \\
\hline bR-QD-MBP & 0.547 \\
\hline bR-QD-MBP-QSY9 & 0.254 \\
\hline bR-QDMSP-QSY9-Maltose & 0.496 \\
\hline
\end{tabular}

From these results the first demonstration of a bR-QD bionanosensing substrate is shown. It was found that the binding of the dark quencher to the MBP resulted in $53.6 \%$ decrease in the bR photovoltaic peak, reducing the peak output from $0.547 \mathrm{mV}$ to $0.254 \mathrm{mV}$. The reduction in bR photovoltage due to the presence of the $\beta$-CD-QSY9 dark quencher bound to MBP correlates closely to the reduction in QD emission due to the same binding, which resulted in a 49\%-50\% reduction in QD emission. Upon the incubation of 
the bR-QD functionalized electrode with $3 \mathrm{mM}$ maltose, the bR photovoltaic output subsequently rose by $95 \%$. This increase in output is much larger than the $14 \%-17 \%$ QD emission increase seen previously for a $1 \mathrm{mM}$ and $10 \mathrm{mM}$ maltose addition, respectively. The large magnitude of change due to $3 \mathrm{mM}$ maltose addition is promising that much lower maltose levels can be detected. 


\subsection{Summary and Conclusions}

The goal of this work was to gain an understanding of the nano-scale interactions of the optical protein bacteriorhdodopsin and colloidal semicondunctor quantum dots, with the purpose of developing a new type of bio-nano hybrid substrate for biosensing applications. Specifically it has been shown that a direct energy transfer relationship exists in the bacteriorhodopsin/quantum dot hybrid pair system and that these nano-scale interactions can be utilized as a generic sensing substrate.

This work spans from the isolation of the protein to its application in a novel biosensing system. A high yield bacteriorhodopsin production and purification scheme was first initiated, yielding purple membrane of greater than $95 \%$ purity. Once created, this purple membrane was integrated onto an optically transparent electrode via electrophoretic sedimentation, where it serves as a photoactive material producing a measurable electrical output upon light activation. The deposition technique and the amplifier construction were optimized for maximal protein photovoltaic performance.

To be utilized as a photoactive material in the proposed nano-scale sensing array with distinct sensing pixels, a protein activation source needed to be integrated that could be localized to each individual sensing pixel. To accomplish this requirement the integration of colloidal semiconductor quantum dots with bacteriorhodopsin was explored. This work showed that a quantum dot solution was capable of photonically activating the bacteriorhodopsin photocycle. Further developements uncovered that the integration of a single quantum dot monolayer deposited in nano-scale proximity to the bacteriorhodopsin electrode yielded a large increase in the protein photovoltaic output that is difficult to justify by photonic activation alone, suggesting non-photonic energy transfer mechanisms.

Detailed investigations into other potential energy transfer mechanisms discovered that a fluorescent resonance energy transfer relationship exists in a bR-QD hybrid system. 
Studies of QD-bR (PM fragment form) interactions in aqueous suspension found that a dipole separation distance of 3-4nm yielded a 55\% reduction in QD emission and a 7.5$9 \mathrm{~nm}$ separation distance yielded a 32\% reduction in QD emission. Delipidating the PM fragments to obtain bR monomers yielded a more optimal $88 \%$ and $49 \%$ reduction in QD emission when linked at $3-4 \mathrm{~nm}$ and $7.5-9 \mathrm{~nm}$ separation distances, respectively. The results of the optimized bR monomer system closely matched theoretical predictions, confirming that this energy transfer phenomena is largely distance dependent, in the sub$10 \mathrm{~nm}$ realm. Excited state single photon counting studies also confirmed a direct energy transfer relationship, showing QD lifetimes of 13.9ns, 11.6ns, and 5.6ns for the QD control, QD-bR (PM fragment form) and QD-bR (monomer form) when linked at 3-4nm separation, respectively. Reduction in the QD lifetime when directly linked in nano-scale proximity to bR confirms that the energy from the QDs excited electrons are transferred directly to the bR retinal.

Deposition of the hybrid system with nano-scale control has allowed for the utilization of this energy transfer phenomena as a modulation point for a functional biosensor prototype. Hybrid QD-bR multi-layered films were created using ionic self assembled monolayer techniques and it was revealed that quantum dots have the ability to activate the bacteriorhodopsin photocycle through both photonic and non-photonic energy transfer mechanisms. Studies confirmed that functional electrodes consisting of a single monolayer of each bR and QD material could be created, however optimal energy transfer could be achieved between a 2 monolayer bR base electrode topped by a single monolayer of QDs.

A biosensing prototype was created in which the energy transfer relationship is altered upon target binding. Specifically a single monolayer of maltose binding protein was ionically deposited onto a hybrid bR-QD bilayer electrode, serving as the receptor molecule. Introduction of a dark quencher molecule, which was designed for MBP binding affinity, ultimately FRET coupled to the QD and reduced the amount of energy available for the $\mathrm{bR}$ molecule. This resulted in 54\% reduction in the bR photovoltaic output. Introducing $3 \mathrm{mM}$ maltose into the system, which has a higher binding affinity for 
MBP than the dark quencher, displaces the dark quencher molecule and restores the QDbR energy transfer relationship. This is evident by a return in the bR photovoltaic output to $91 \%$ of its original value, demonstrating the applicability of a QD-bR hybrid system for sensor applications.

The electrical nature of this sensing substrate will allow for its efficient integration into a nanoelectronics array form, potentially leading to a small-low power sensing platform for remote toxin detection applications. The initial proof-of-concept sensor demonstrated the functionality of this technology and justifies further investigations into using this system for the detection of a wide array of target molecules, including toxins.

\subsection{SUGGESTIONS FOR FUTURE WORK}

This work has been an initial effort into the characterization of the bR-QD interactions, their integration into nano-scale hybrid electrodes, and demonstration of the hybrid systems capabilities as a biosensing device. The discoveries and techniques outlined above lay the ground-work for the development of a generic bR-QD hybrid sensing array. Future work will need to be performed to further optimize this system and integrate it into a deployable device.

To create a sensor based on the energy transfer interactions between quantum dots and bacteriorhdopsin in monlayer film form, it will be important to understand how close the dried film system matches the theoretical FRET efficiency model shown in Figure 77. This will require creating a QD-bR hybrid electrode with varying separation distances between the $\mathrm{bR}$ and QD monolayers. One possible method of attaining the desired separation distance is by stacking consecutive positive and negative polyelectrolyte layers to attain a desired thickness. It has been estimated that each polyelectrolyte layer is about $0.5 \mathrm{~nm}$ thick, so an approximate spacer distance can be attained. An example setup would be to create a bR base monolayer, deposit the polyelectrolytes to the desired 
thickness, and finally add the QD monolayer. A cuvette setup to hold each sample at an exact location in the fluorospectrometer for QD emission measurements would be needed. A comparison of the reduced QD emission levels at each separation distance will give a rough estimate of FRET efficiency. Ideally the QD lifetime would be measured in each sample to isolate energy transfer.

Since the I-SAM layering technique requires an additional polyelectrolyte material to create multi-layered films, studies should be performed on the bR/QD hybrid system efficacy without the presence of the additional polyelectrolyte spacer material. One method of exploring this is through the use of Langmuir-Blodgett (LB) monolayer deposition techniques. Previous work has demonstrated the ability to deposit bR and QD monolayers separately using the LB technique, thus it is feasible that these techniques could be implemented in a dual-trough LB setup to construct multi-layered bR/QD electrodes. A secondary method of approaching this could be to create positively charged QDs for deposition directly on top of the negatively charged bR, still using the I-SAM technique. This technique would eliminate the general polyelectrolyte spacer layer, however creation of positively charged QDs requires the absorption of a positive polyelectrolyte material to the negatively charged $\mathrm{QD}$, thus additional polyelectrolyte material would still be present.

To further characterize the capabilities of $\mathrm{bR}$ in a field-deployable device, additional studies should be focused on the effects of temperature on the bR photovoltaic output. Initial work has been performed showing the effect of elevated and reduced temperatures on the bR output and recovery, however further work will need to be performed to confirm these results and provide justifications for the bR property alterations.

Work will also need to be performed focused on maximizing the bR output per unit area. One potential method to increase the photovoltaic response of a bR monolayer film and ultimately increase device sensitivity is to utilize oriented bR monomers instead of PM fragments as the base material. This will eliminate the associated lipids of PM fragment films and increase the amount of bR molecules present per unit area. Theoretically the 
bR monomers will still display a strong negative dipole at elevated $\mathrm{pH}$ levels and could be deposited with the I-SAM technique. Studies comparing bR monomer electrodes to PM fragement electrodes would elucidate the impact of a higher bR concentration. Since the final nano-scale sensor array might have limited gate electrode area for bR activation, it might be necessary to maximize bR density.

Once the QD-bR hybrid system is ready to be applied on a transistor gate, work will need to be undertaken to integrate the I-SAM or LB monolayer deposition techniques with the dissertation work performed by Chris Anton and Karl Walczak at the Michigan Technological University. Chris Anton has shown the ability to pattern functional bR electrodes via photolithographic techniques, while Karl Walczak has demonstrated the capability of activating a single electron transistor with the bR photovoltaic response. Literature has shown the I-SAM technique is capable of being applied with patterning techniques, so it is feasible to pattern functional bR-QD electrodes onto the gate of an SET via photolithographic techniques. 


\section{$\underline{\text { References }}$}

1. Augerson, W., A Review of the Scientific Literature as it Pertains to Gulf War Illnesses. Volume 5: Chemical and Biological Agents. 2000: RAND Research. 316.

2. Wiener, S. and R. Hoffman, Nerve Agents: A Comprehensive Review. Journal of Intensive Care Medicine, 2004. 19(1): p. 22-37.

3. Gieray, R.A., et al., Real-Time Detection of Individual Airborne Bacteria. Journal of Microbiological Methods, 1997. 29(3): p. 191-199.

4. Hinz, K., et al., Data Processing in On-line Laser Mass Spectrometry of Inorganic, Organic, or Biological Airborne Particles. Journal of the American Society for Mass Spectrometry, 1999. 10(7): p. 648-660.

5. Parker, A., When Every Second Counts: Pathogen Identification in Less than a Minute, in Science and Technology Review. 2003.

6. Tobias, H.J., et al., Bioaerosol Mass Spectrometry for Rapid Detection of Individual Airborne Mycobacterium tuberculosis H37Ra Particles. Applied and Environmental Microbiology, 2005. 71(10): p. 6086-6095.

7. Kock, C. and B. Mathur, Neuromorphic Vision Chips. IEEE Spectrum, 1996: p. 38-46.

8. W.H.O. Tuberculosis Facts Sheet. 2007 [cited May 22nd, 2007]; Available from: http://www.who.int/mediacentre/factsheets/fs104/en/.

9. Biowarn, BioWarn, LLC and the SmartSenseTM System. 2006.

10. Oleynik, V.A., Methodology and Apparatus for the Detection of Biological Substances, USPTO, Editor. 2005, Biowarn LLC: USA.

11. Luther, G. Perspective on Nosocomial Infection Control. 2005 [cited; Available from: http://www.biowarnllc.com/downloads/.

12. Mahan, C., Sandia National Laboratories' portable uChemLab Biological Agent Detection Sensor, HSARPA, Editor. 2005.

13. Haupts, U., J. Tittor, and D. Oesterhelt, Closing in on bacteriorhodopsin: Progress in understanding the molecule. Annu. Rev. Biophys. Biomol. Struc., 1999. 28: p. 367-399.

14. Hwang, S.-B. and W. Stoeckenius, Purple membrane vesicles: morphology and proton translocation. J. Membrane Biol., 1977. 33: p. 325-350.

15. Lanyi, J.K., Proton transfers in the bacteriorhodopsin photocycle. Biochimica et Biophysica Acta (BBA) - Bioenergetics, 2006. 1757(8): p. 1012-1018.

16. Haupts, U., et al., General Concept for Ion Translocation by Halobacterial Retinal Proteins: The Isomerization/Switch/Transfer (IST) Model. Biochemistry, 1997. 36(1): p. 2-7.

17. Váró, G., Analogies between halorhodopsin and bacteriorhodopsin. Biochimica et Biophysica Acta (BBA) - Bioenergetics, 2000. 1460(1): p. 220-229.

18. Oesterhelt, D., The structure and mechanism of the family of retinal proteins from halophilic archaea. Current Opinion in Structural Biology, 1998. 8(4): p. 489500 . 
19. Brown, L.S., Proton Transport Mechanism of Bacteriorhodopsin as Revealed by Site-Specific Mutagenesis and Protein Sequence Variability. Biochemistry (Moscow), 2001. 66(11): p. 1249-1255.

20. Stuart, J.A., et al., Preparation, purification and modification of bacteriorhodopsin for use in protein-based optical devices.

21. Hampp, N., Bacteriorhodopsin as a Photochromic Retinal Protein for Optical Memories. Chem. Rev., 2000. 100: p. 1755-1776.

22. Miyasaka, T., K. Koyama, and I. Itoh, Quantum Conversion and Image Detection by a Bacteriorhodopsin-Based Artificial Photoreceptor. Science, 1992.

255(5042): p. 342-344.

23. Crittenden, S., et al., Humidity-dependent open-circuit photovoltage from a bacteriorhodopsin/indium tin oxide bioelectronic heterostructure.

Nanotechnology, 2003. 14: p. 562-565.

24. Wang, W., G. Knopf, and A. Bassi, Photoelectric properties of a detector based on dried bacteriorhodopsin film. Biosensors \& Bioelectronics, 2006. 21: p. 13091319.

25. Varo, G. and L. Keszthelyi, Photoelectric signals from dried oriented purple membranes of halabacterium halobium. Biophys. Journal, 1983. 43: p. 47-51.

26. Wang, J.-P., et al., Molecular mechanism of the differential photoelectric response of bacteriorhodopsin. J. Phys. Chem, 1997. 101: p. 3420-3423.

27. Takamatsu, S., et al., Biomolecular image sensor of bacteriorhodopsin patterned by electrodeposition. MEMS, 2005. 18th IEEE International Conference: $p$. 847-850.

28. Ganea, C., et al., The role of water in the extracellular half channel of bacteriorhodopsin. Biophys. Journal, 1997. 73: p. 2718-2725.

29. Groma, G.I., et al., Photocyle of Dried Acid Purple Form of Bacteriorhodopsin. Biophys. Journal, 2001. 81: p. 3432-3441.

30. Robertson, B. and E. Lukashev, Rapid pH Change due to Bacteriorhodopsin Measured with a Tin-Oxide Electrode. Biophys. Journal, 1995. 68: p. 1507-1517.

31. Liu, S.Y., M. Kono, and T.G. Ebrey, Effect of pH Buffer Molecules on the LightInduced Currents from Oriented Purple Membrane. Biophys. Journal, 1991. 60: p. 204-216.

32. Bertoncello, P., et al., Bacteriorhodopsin-based langmuir-schaefer for solar energy capture. IEEE Transactions on NanoBioScience, 2003. 2(2): p. 124-132.

33. Miyasaka, T. and K. Koyama, Image Sensing and Processing by a Bacteriorhodopsin-Based Artificial Photoreceptor. Applied Optics, 1993. 32(31): p. 6371-6379.

34. Birge, R., Photophysics and Molecular Electronic Applications of the Rhodopsins. Ann. Rev. Phys. Chem., 1990. 41: p. 683-733.

35. Bhattacharya, P., et al., Monolithically intergrated bacteriorhodopsin-GaAs fieldeffect transitor photoreceiver. Optics Letters, 2002. 27(10): p. 839-841.

36. Crittenden, S., et al., Soft lithography based micron-scale electrophoretic patterning of purple membrane. J. Micromech. Microeng., 2005. 15: p. 14941497. 
37. London, E. and H.G. Khorana, Denaturation and Renaturation of

Bacteriorhodopsin in Detergents and Lipid-Detergent Mixtures. Journal of Biological Chemistry, 1981. 257(12): p. 7003-7011.

38. Marie, M.1., P. Champeil, and J.V. Moller, Interaction of Membrane Proteins and Lipids with Solubilizing Detergents. Biochimica et Biophysica Acta, 2000. 1508: p. 86-111.

39. Guzelian, A.A., et al., Colloidal chemical synthesis and characterization of InAs nanocrystal quantum dots. Applied Physics Letters, 1996. 69(10): p. 1432-1434.

40. Peng, Z. and X. Peng, Formation of high-quality CdTe, CdSe, and CdS nanocrystals using CdO as precursor. J. Am. Chem. Soc., 2001. 123: p. 183-184.

41. Medintz, I.L., et al., Self-assembled nanoscale biosensors based on quantum dot FRET donors. Nature Materials, 2003. 2: p. 630-638.

42. Jovin, T.M., Quantum dots finally come of age. Nature Biotechnology, 2003. 21: p. 32-33.

43. Michalet, X., et al., Quantum Dots for Live Cells, in Vivo Imaging, and Diagnostics. Science, 2005. 307(5709): p. 538-544.

44. Gao, X., et al., In Vivo Molecular and Cellular Imaging with Quantum Dots. Current Opinion in Biotechnology, 2005. 16: p. 63-72.

45. Chan, W.C.W., et al., Luminescent quantum dots for multiplexed biological detection and imaging. Current Opinion in Biotechnology, 2002. 13(1): p. 40-46.

46. Nozik, A.J., Quantum dot solar cells. Physica E: Low-dimensional Systems and Nanostructures, 2002. 14(1-2): p. 115-120.

47. Robel, I., et al., Quantum Dot Solar Cells. Harvesting Light Energy with CdSe Nanocrystals Molecularly Linked to Mesoscopic TiO2 Films. J. Am. Chem. Soc., 2006. 128(7): p. 2385-2393.

48. Plass, R., et al., Quantum Dot Sensitization of Organic-Inorganic Hybrid Solar Cells. J. Phys. Chem. B, 2002. 106(31): p. 7578-7580.

49. Loss, D. and D.P. DiVincenzo, Quantum computation with quantum dots. Physical Review A, 1998. 57(1): p. 120.

50. Klein, D., et al., A single-electron transistor made from a cadmium selenide nanocrystal. Nature, 1997. 389: p. 699-701.

51. Walter, G., et al., Coupled InP quantum-dot In GaP quantum well InP--InGaP-In $(A l G a) P$--InAlP heterostructure diode laser operation. Applied Physics Letters, 2001. 79(20): p. 3215-3217.

52. Heinrichsdorff, F., et al., High-power quantum-dot lasers at $1100 \mathrm{~nm}$. Applied Physics Letters, 2000. 76(5): p. 556-558.

53. Hines, M. and P. Guyot-Sionnest, Synthesis and characterization of strongly luminescing ZnS-capped CdSe nanocrystals. J. Phys. Chem., 1996. 100: p. 468471.

54. Qu, L. and X. Peng, Control of Photoluminescence Properties of CdSe Nanocrystals in Growth. J. Am. Chem. Soc., 2002. 124(9): p. 2049-2055.

55. Xiaogang, P., Green Chemical Approaches toward High-Quality Semiconductor Nanocrystals. Chemistry - A European Journal, 2002. 8(2): p. 334-339.

56. Qu, L., Z.A. Peng, and X. Peng, Alternative Routes toward High Quality CdSe Nanocrystals. Nano Lett., 2001. 1(6): p. 333-337. 
57. Ziegler, J., et al., High-Quality ZnS Shells for CdSe Nanoparticles: Rapid Microwave Synthesis. Langmuir, 2007. 23(14): p. 7751-7759.

58. Sark, W.v., et al., Photooxidation and Photobleaching of Single CdSe/ZnS Quantum Dots Probed by Room-Temperature Time-Resolved Spectroscopy. J. Phys. Chem, 2001. 105: p. 8281-8284.

59. Bieri, C., et al., Micropatterned immobilization of a $G$ protein-coupled receptor and direct detection of $G$ protein activation. Nature Biotechnology, 1999. 17(11): p. 1105-1108.

60. Lidke, D.S. and D.J. Arndt-Jovin, Imaging takes a quantum leap. Physiology, 2004. 19: p. 322-325.

61. Jaiswal, J.K., et al., Long-term multiple color imaging of live cells using quantum dot bioconjugates. Nature Biotechnology, 2003. 21: p. 47-51.

62. Sark, W.v., et al., Blueing, Bleaching, and Blinking of Single CdSe/ZnS Quantum Dots. Chem. Phys. Chem., 2002. 3: p. 871-879.

63. Nirmal, M., et al., Fluorescence intermittency in single cadmium selenide nanocrystals. Nature, 1996. 383(6603): p. 802-804.

64. Evident. Quantum Dot Based Evitags. 2007 [cited March 12th, 2007]; Available from: http://www.evidenttech.com/life_sciences/evitags/quantum-dot-evitagintroduction.php.

65. Stryer, L. and R.P. Haugland, Energy Transfer: A Spectroscopic Ruler. Biochemistry, 1967. 58: p. 719-726.

66. Jares-Erijman, E.A. and T.M. Jovin, FRET imaging. Nature Biotechnology, 2003. 21(11): p. 1387-1395.

67. Santagelo, P., et al., Dual FRET molecular beacons for mRNA detection in living cells. Nucleic Acids Research, 2004. 32(6).

68. Polozova, A. and B. Litman, Cholesterol Dependent Recruitment of di22:6-PC by a G Protein-Coupled Receptor into Lateral Domains. Biophys. Journal, 2000. 79: p. 2632-2643.

69. Rehorek, M., Fluorescence Energy Transfer from Diphenylhexatriene to Bacteriorhodopsin in Lipid Vesicles. Biophys. Journal, 1983. 43: p. 39-45.

70. Dumas, F., et al., Molecular Sorting of Lipids by Bacteriorhodopsin in Dilauroylphosphatidylcholine/Distearoylphosphatidylcholine Lipid Bilayers. Biophys. Journal, 1997. 73: p. 1940-1953.

71. Dancshazy, Z., Z. Tokaji, and A. Der, Bleaching of Bacteriorhodopsin by Continuous Light. FEBS Letters, 1999. 450: p. 154-157.

72. Clapp, A.R., et al., Fluorescence resonance energy transfer between quantum dot donors and dye-labeled protein acceptors. J. Am. Chem. Soc., 2004. 126: p. 301310.

73. Shimizu, K.T., et al., Surface-Enhanced Emission from Single Semiconductor Nanocrystals. Physical Review Letters, 2002. 89(11): p. 117401.

74. Dyadyusha, L., et al., Quenching of CdSe quantum dot emission, a new approach for biosensing. Chem. Commun., 2005: p. 3201-3203.

75. Oh, E., et al., Inhibition Assay of Biomolecules based on Fluorescence Resonance Energy Transfer (FRET) between Quantum Dots and Gold Nanoparticles. J. Am. Chem. Soc., 2005. 127(10): p. 3270-3271. 
76. Li, J., et al., Ultrasensitive Optical DNA Biosensor Based on Surface Immobilization of Molecular Beacon by a Bridge Structure. Analytical Sciences, 2001. 17: p. 1149-1153.

77. Paloniemi, H., et al., Layer-by-Layer Electrostatic Self-Assembly of Single-Wall Carbon Nanotube Polyelectrolytes. Langmuir, 2006. 22(1): p. 74-83.

78. Kalsin, A.M., et al., Electrostatic Self-Assembly of Binary Nanoparticle Crystals with a Diamond-Like Lattice. Science, 2006. 312(5772): p. 420-424.

79. Pei, R., et al., Assembly of Alternating Polycation and DNA Multilayer Films by Electrostatic Layer-by-Layer Adsorption. Biomacromolecules, 2001. 2(2): p. 463468.

80. Kristie M. Lenahan, Y.-X.W.Y.L.R.O.C.J.R.H.D.M.C.F., Novel Polymer Dyes for Nonlinear Optical Applications Using Ionic Self-Assembled Monolayer Technology. Advanced Materials, 1998. 10(11): p. 853-855.

81. He, J.A., et al., Photoelectric Properties of Oriented Bacteriorhodopsin/Polycation Multilayers by Electrostatic Layer-by-Layer Assembly. J. Phys. Chem. B, 1998. 102(36): p. 7067-7072.

82. He, J.-A., et al., Oriented bacteriorhodopsin/polycation multilayers by electrostatic layer-by-layer assembly. Langmuir, 1997. 14: p. 1674-1679.

83. He, J.-A., et al., Bacteriorhodopsin thin film assemblies-Immobilization, properties, and applications. Advanced Materials, 1999. 11(6): p. 435-445.

84. He, T., et al., Bacteriorhodopsin monolayers for optoelectronics: orientation and photoelectric response on solid supports. Advanced Materials, 2005. 17(8): p. 1023-1027.

85. Miercke, L.J., et al., Purification of bacteriorhodopsin and characterization of mature and partially processed forms. J. Biol. Chem., 1989. 264(13): p. 75317535.

86. Ross, P.E., et al., Isoelectric focusing studies of bacteriorhodopsin. Biochimica et Biophysica Acta (BBA) - General Subjects, 1989. 991(1): p. 134-140.

87. Cordero, S., et al., Photo-activated luminescence of CdSe quantum dot monolayers. J. Phys. Chem, 2000. 104: p. 12137-12142.

88. Uematsu, T., S. Maenosono, and Y. Yamaguchi, Photoinduced fluorescence enhancement in CdSe/ZnS quantum dot monolayers: Influence of substrate. Applied Physics Letters, 2006. 89: p. 03910-3.

89. Uematsu, T., J. Kimura, and Y. Yamaguchi, The reversible photoluminescence enhancement of a CdSe/ZnS nanocrystal thin film. Nanotechnology, 2004. 15: p. 822-827.

90. S. Coe-Sullivan, J.S.S.W.K.W.M.G.B.V.B. and cacute, Large-Area Ordered Quantum-Dot Monolayers via Phase Separation During Spin-Casting. Advanced Functional Materials, 2005. 15(7): p. 1117-1124.

91. Jaffar, S., et al., Layer-by-layer surface modification and patterned electrostatic deposition of quantum dots. Nano Letters, 2004. 4(8): p. 4.

92. Constantine, C.A., et al., Layer-by-Layer Biosensor Assembly Incorporating Functionalized Quantum Dots. Langmuir, 2003. 19(23): p. 9863-9867.

93. Vamsi, K.K., et al., Emission properties of colloidal quantum dots on polyelectrolyte multilayers. Nanotechnology, 2006(16): p. 4117. 
94. Chan, W.C., et al., Quantum Dot Bioconjugates for Ultrasensitive Nonisotopic Detection. Science, 1998. 281(5385): p. 2016-2018.

95. Jaffar, S., et al., Layer-by-Layer Surface Modification and Patterned Electrostatic Deposition of Quantum Dots. Nano Lett., 2004. 4(8): p. 1421-1425.

96. Kagan, C., et al., Electronic energy transfer in CdSe quantum dot solids. Physical Review Letters, 1996. 76(9): p. 1517-1520.

97. Anton, C., Photolithography based patterning of bacteriorhodopsin films, in Mechanical Engineering-Engineering Mechanics. 2008, Michigan Technological University: Houghton. p. 170.

98. Kovacs, I. and G. Varo, Dielectric dispersion and protonic conduction in hydrated purple membrane. Acta. Biochim. Biophys. Hung., 1988. 23: p. 265270.

99. Bridgen, J. and I.D. Walker, Photoreceptor protein from the purple membrane of halobacterium halobium. Molecular weight and retinal binding site. Biochemistry, 1976. 15 (4): p. 792-798.

100. Walczak, K., Immobilizing bacteriorhdodopsin on a single electron transistor, in Mechanical Engineering-Engineering Mechanics. 2008, Michigan Technological University: Houghton. p. 150.

101. Rayfield, G.W., Temperature dependence of photovoltages generated by bacteriorhodopsin. Biophys. J., 1985. 48(1): p. 111-115.

102. Janovjak, H., et al., Unfolding pathways of native bacteriorhodopsin depend on temperature. The EMBO Journal, 2003. 22(19): p. 5220-5229.

103. Kalisky, O., J. Feitelson, and M. Ottolenghi, Photocehmistry and Fluorescence of Bacteriorhodopsin Excited in Its 280-nm Absorption Band. Biochemistry, 1981. 20: p. 205-209.

104. Weber, P.C., et al., Structural origins of high-affinity biotin binding to streptavidin. Science, 1989. 243(4887): p. 85-88.

105. Anders Holmberg, A.B.O.N.M.L.J.L.M.U., The biotin-streptavidin interaction can be reversibly broken using water at elevated temperatures. ELECTROPHORESIS, 2005. 26(3): p. 501-510.

106. Oh, E., et al., Inhibition Assay of Biomolecules based on Fluorescence Resonance Energy Transfer (FRET) between Quantum Dots and Gold Nanoparticles. J. Am. Chem. Soc., 2005. 127: p. 3270-3271.

107. Livnah, O., et al., Three-dimensional structures of avidin and the avidinbiotin complex. Biochemstry, 1993. 90: p. 5076-5080.

108. Qin, H., Z. Liu, and S.-f. Sui, Two-Dimensional crystallization of avidin on biotinylated lipid monolayers. Biophysical Journal, 1995. 68: p. 2493-2496.

109. Xu, C., B. Xing, and J. Rao, A self-assembled quantum dot probe for detecting beta-lactamase activity. Biochemical and Biophysical Research Communications, 2006. 344: p. 931-935.

110. Cui, Y., et al., Nanowire Nanosensors for Highly Sensitive and Selective Detection of Biological and Chemical Species. Science, 2001. 293(5533): p. 1289-1292.

111. Chen, D.-1., et al., Oriented assembly of purple membrane on solid support mediated by molecular recognition. J. Phys. Chem, 2003. 107: p. 3598-3605. 
112. Heeg, B., et al., Bacteriorhodopsin as a chemical and biological sensor. Proceedings of SPIE, 2003. 5085.

113. Sharma, M.K., H. Jatteni, and J. M. Lane Gilchrist, Bacteriorhodopsin conjugates as anchors for supported membranes. Bioconjugate chem. , 2004. 15: p. 942-947.

114. Winder, E., et al., Chemical and biological sensing utilizing fused bacteriorhodopsin protein hybrids. Proc. 26th Army Science Conference, 2008.

115. Min, J., et al., Photocurrent of bacteriorhodopsin films deposited by electrophoretic method. Thin Solid Films, 1998. 327-329: p. 698-702.

116. He, J.-A., et al., Oriented bacteriorhodopsin/polycation multilayers by electrostatic layer-by-layer assembly. Langmuir, 1998. 14: p. 1674-1679.

117. He, J.-A., et al., Photoelectric properties of oriented bacteriorhodopsin/polycation multilayers by electrostatic layer-by-layer assembly. J. Phys. Chem, 1998. 102: p. 7067-7072.

118. Darst, S.A., et al., Two-dimensional crystals of streptavidin on biotinylated lipid layers and their interactions with biotinylated macromolecules. Biophys. Journal, 1991. 59: p. 387-396.

119. Huang, T.J. and J.R. Waldeisen, Biologically inspired energy: harnessing molecular functionality towards nanosystemic design. Nanomedicine, 2006. 1(3): p. 369-372.

120. Su, T., et al., Asymmetric distribution of biotin labeling on the purple membrane. Journal of Photochemistry and Photobiology, 2008. 92(2): p. 123-127.

121. Nakajima, N. and Y. Ikada, Mechanism of Amide Formation by Carbodiimide for Bioconjugation in Aqueous Media. Bioconjugate Chem., 1995. 6(1): p. 123-130.

122. Staros, J., R. Wright, and D. Swingle, Enhancement by $N$ hydroxysulfosuccinimide of water-soluble carbodiimide-mediated coupling reactions. Analytical Biochemistry, 1986. 156(1): p. 220-222.

123. Rental, R. and M. Perez, Bleaching of purple membrane with O-substituted hydroxylamines. Photochem. Photobiol., 1982. 36: p. 345-348.

124. Medintz, I.L., et al., A fluorescence resonance energy transfer-derived structure of a quantum dot-protein bioconjugate nanoassembly. PNAS, 2004. 101(26): p. 9612-9617.

125. Birge, R.R., et al. Bioelectronics, three-dimensional memories and hybrid computers. in Electron Devices Meeting, 1994. IEDM '94. Technical Digest., International. 1994.

126. Ewing, R.L., et al. Exploring the biocomputing frontier. in Circuits and Systems, 2005. 48th Midwest Symposium on. 2005.

127. Nicolini, C., et al., Toward bacteriorhodopsin based photocells. Biosensors and Bioelectronics, 1999. 14(4): p. 427-433.

128. Fehr, M., W.B. Frommer, and S. Lalonde, Visualization of maltose uptake in living yeast cells by fluorescent nanosensors. Proceedings of the National Academy of Sciences of the United States of America, 2002. 99(15): p. 98469851.

129. Spurlino, J.C., G.Y. Lu, and F.A. Quiocho, The 2.3-A resolution structure of the maltose- or maltodextrin-binding protein, a primary receptor of bacterial active transport and chemotaxis. J. Biol. Chem., 1991. 266(8): p. 5202-5219. 
130. Vyas, N.K., M.N. Vyas, and F.A. Quiocho, Sugar and signal-transducer binding sites of the Escherichia coli galactose chemoreceptor protein. Science, 1988. 242(4883): p. 1290-1295.

131. Sharff, A., L. Rodseth, and F.A. Quiocho, Refined 1.8-A structure reveals themode of binding of B-cyclodextrin to the maltodextrin binding protein. Biochemistry, 1993. 32: p. 10553-10559.

132. Ferenci, T. and U. Klotz, Affinity chromatographic isolation of the periplasmic maltose binding protein of Escherichia coli. FEBS Letters, 1978. 94(12): p. 213217.

133. Kellerman, O.K. and T. Ferenci, Maltose-binding protein from Escherichia coli. Methods Enzymol., 1982. 75: p. 459-463.

134. Nossal, N.G. and L.A. Heppel, The Release of Enzymes by Osmotic Shock from Escherichia coli in Exponential Phase. J. Biol. Chem., 1966. 241(13): p. 30553062 .

135. Medintz, I.L. and J.R. Deschamps, Maltose-binding protein: a versatile platform for prototyping biosensors. Current Opinion in Biotechnology, 2006. 17(1): p. $17-$ 27.

136. Dahl, M.K. and M.D. Manson, Interspecific reconstitution of maltose transport and chemotaxis in Escherichia coli with maltose-binding protein from various enteric bacteria. J. Bacteriol., 1985. 164(3): p. 1057-1063. 


\section{Appendix A: Permissions from Publishers}

\section{A.1: PeRmissions FOR Figure 3.}

Dear Mark:

You may use the schematic. Please credit Lawrence Livermore National Laboratory in your dissertation. Please let me know if you have any further questions.

Sincerely,

Anne

To:1lnlweb@1lnl.gov

Subject: LLNL Web Comment

X-Mailer: LLNL/AIS Email Sender

Sender: owner-1lnlweb@lists.llnl.gov

Name: Mark Griep

E-mail:mhgriep@mtu.edu

Subject: other

Comment: Hi,

I'm writing to ask for permission to use a figure from your magazine in my $\mathrm{PhD}$ dissertation. The figure is from the article:

Parker, A., When Every Second Counts: Pathogen Identification in

Less than a Minute, in Science and Technology Review. September, 2003.

Specifically I would like to refer to the first figure of the BAMS aerosol schematic.

My $\mathrm{PhD}$ work on biosensors is a $\mathrm{DoD}$ funded contract administered by the Army Research Lab. Please let me know if this is OK. Thanks for your help

-Mark

This message was generated by the LLNL Web Comments form. http://www.llnl.gov/llnl/contact.jsp

Anne M. Stark

Sr. Public Information Officer 
Lawrence Livermore National Laboratory

7000 East Avenue, L-3

Livermore, CA 94550

(925) 422-9799

\title{
A.2: Permissions for Figure 4.
}

Yes. Permission applies to all educational uses. Good luck on your PhD process.

\author{
Stephanie Holinka \\ Media Relations \& Communications \\ Sandia National Laboratories \\ slholin@sandia.gov (preferred method) \\ office phone: (505)료-9227
}

-----Original Message-----

From: Mark Griep [mailto:mhgriep@mtu.edu]

Sent: Saturday, October 25, 2008 12:38 AM

To: WEBMASTER

Subject: Permission to use image in $\mathrm{PhD}$ dissertation

Hi,

I'm working on a DoD contract with the Michigan Technological University and am writing to ask permission to use one of Sandia's images in my dissertation. The image is located at:

http://www.sandia.gov/news-center/news-releases/2003/mat-chem/chempartners.html

It's the image of the orange hand-held micro-chemlab system. Permission is given under the picture for media to use this image, but I just wanted to make sure this permission applies to my needs as well. If there is someone else I should contact for this, please let me know. Thanks for your help!

-Mark

\section{A.3: PERMissions FOR Figure 10 AND Figure 11.}

To whom it may concern,

I'm writing my $\mathrm{PhD}$ dissertation titled: "Integration of Quantum Dots with the Optical 
Protein Bacteriorhodopsin" for the Michigan Technological University. I am writing you to ask permission to two images from your publications. The images are in the article:

Bhattacharya, P., et al., Monolithically intergrated bacteriorhodopsin-GaAs field-effect transitor photoreceiver. Optics Letters, 2002. 27(10): p. 839-841.

Specifically I'm asking permission to use Figures 1 (pg.839) and 2(a) (pg.840) for reference in the $\mathrm{PhD}$ dissertation. If there is someone else I should contact for this or a specific form to use, please let me know. Thanks for your help!

-Mark Griep

Tel: $906-281-2939$

Fax: $\underline{906-487-2822}$

Dear Mark Griep,

Below is permission for your request.

Sincerely,

Susannah Lehman

The Optical Society of America considers reproduction of small portions of its copyrighted material such as you request below to be Fair Use under U.S. Copyright Law. It is requested that a complete citation of the original material be included in any publication. If you require any confirmation or permission other than what this e-mail grants, please feel free to contact me.

Susannah Lehman

Authorized Agent

OSA

\section{A.4: PERMisSions For Figure 12 AND Figure 13.}

To whom it may concern

I am preparing a work entitled

"Integration of quantum dots with the optical protein bacteriorhodopsin."

to be published as my PhD dissertation with the Michigan Technological University. 
I would appreciate permission to reproduce the following item(s) in both print and electronic editions of the Journal and in all subsequent future editions of the Journal, any derivative products and in publisher authorized distribution by third party distributors, aggregators and other licensees such as abstracting and indexing services. I should be grateful for nonexclusive perpetual world rights in all languages and media. Unless you indicate otherwise, I will use the complete reference given below as the credit line.

In case you do not control these rights, I would appreciate it if you could let me know to whom I should apply for permissions.

Crittenden, S., et al., Soft lithography based micron-scale electrophoretic patterning of purple membrane. J. Micromech. Microeng., 2005. 15: p. 1494-1497.

Figure 1, page 1495

Figure 3(a), page 1496

For your convenience a copy of this letter may serve as a release form: the duplicate copy may be retained for your files.

Thank you for your prompt attention to this request.

Yours sincerely

Mark Griep

Tel: 906-281-2939

Fax: 906-487-2822

I/We grant permission for the use of the work as set out above.

Signed:

Date:

On behalf of Publisher: 
PERMISSION TO REPRODUCE AS REQUESTE[

IS GIVEN PROVIDED THAT:

(a) the consent of the author(s) is obtained

(b) the source of the material including authorieditor, title, date and publisher is acknowledgerd.

IOP Publishing Lttd

Dirac House

Temple Back

BRISTOL

BS1 1 BEE

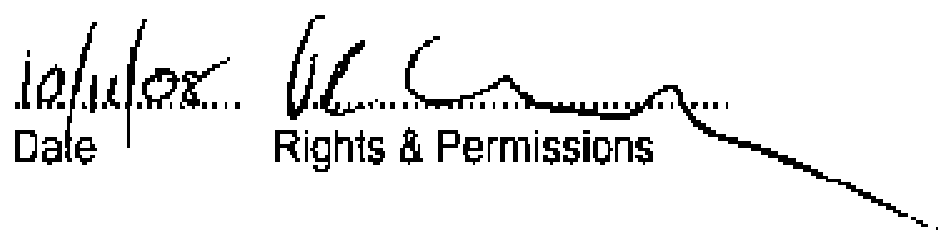

\section{A.5: PeRMisSiONS FOR Figure 18.}

$\begin{array}{ll}\text { Title: } & \begin{array}{l}\text { Bleaching of } \\ \text { bacteriorhodopsin by } \\ \text { continuous light }\end{array} \\ & \begin{array}{l}\text { Zsolt Dancsházy, Zsolt } \\ \text { Tokaji and András Dér }\end{array} \\ \text { Author: } & \text { FEBS Letters } \\ \text { Publication: } & \text { FES } \\ \text { Publisher: } & \text { Elsevier } \\ \text { Date: } & \text { 30 April 1999 } \\ \text { Copyright C } & \text { 1999 Federation of } \\ \text { European Biochemical Societies. } \\ \text { Published by Elsevier B.V. }\end{array}$

Order Completed

Thank you for placing your Rightslink license request for reuse of Elsevier Limited content. It consists of your order details, the terms and conditions provided by Elsevier Limited and the payment terms and conditions.

Get the printable license.

License Number

License date
2063271302128

Nov 06, 2008 
Licensed Chapter Title

Licensed Chapter ID

Licensed content publisher

Elsevier

Licensed content publication

FEBS Letters

Licensed content title

Bleaching of bacteriorhodopsin by continuous light

Licensed content author

Zsolt Dancsházy, Zsolt Tokaji and András Dér

Licensed content date

30 April 1999

Volume number

450

Issue number

$1-2$

Pages

4

Type of Use

Thesis / Dissertation

Portion

Figures/table/illustration/abstracts

Portion Quantity

1

Format

You are the author of this Elsevier article

Both print and electronic

Are you translating?

No

No

Purchase order number

Expected publication date

Jan 2009

Elsevier VAT number

GB 494627212

Permissions price

0.00 USD

Value added tax $0.0 \%$

0.00 USD

Total

0.00 USD

This is a License Agreement between Mark H Griep ("You") and Elsevier ("Elsevier"). The license consists of your order details, the terms and conditions provided by Elsevier, and the payment terms and conditions.

Supplier

Elsevier Limited

The Boulevard,Langford Lane

Kidlington,Oxford,OX5 1GB,UK

Registered Company Number

1982084

Customer name 


\section{Mark H Griep}

Customer address

815 R.L. Smith Bldg

Houghton, MI 49931

License Number

2063271302128

License date

Nov 06, 2008

Licensed content publisher

Elsevier

Licensed content publication

FEBS Letters

Licensed content title

Bleaching of bacteriorhodopsin by continuous light

Licensed content author

Zsolt Dancsházy, Zsolt Tokaji and András Dér

Licensed content date

30 April 1999

Volume number

450

Issue number

$1-2$ 


\section{Pages}

4

Type of Use

Thesis / Dissertation

Portion

Figures/table/illustration/abstracts

Portion Quantity

1

Format

Both print and electronic

You are an author of the Elsevier article

No

Are you translating?

No

Purchase order number

Expected publication date

Jan 2009

Elsevier VAT number

GB 494627212

Permissions price

0.00 USD

Value added tax $0.0 \%$

0.00 USD 
Total

0.00 USD

Terms and Conditions

\section{INTRODUCTION}

1. The publisher for this copyrighted material is Elsevier. By clicking "accept" in connection with completing this licensing transaction, you agree that the following terms and conditions apply to this transaction (along with the Billing and Payment terms and conditions established by Copyright Clearance Center, Inc. ("CCC"), at the time that you opened your Rightslink account and that are available at any time at $<$ http://myaccount.copyright.com $>$ ).

\section{GENERAL TERMS}

2. Elsevier hereby grants you permission to reproduce the aforementioned material subject to the terms and conditions indicated.

3. Acknowledgement: If any part of the material to be used (for example, figures) has appeared in our publication with credit or acknowledgement to another source, permission must also be sought from that source. If such permission is not obtained then that material may not be included in your publication/copies. Suitable acknowledgement to the source must be made, either as a footnote or in a reference list at the end of your publication, as follows:

"Reprinted from Publication title, Vol /edition number, Author(s), Title of article / title of chapter, Pages No., Copyright (Year), with permission from Elsevier [OR APPLICABLE SOCIETY COPYRIGHT OWNER]." Also Lancet special credit - "Reprinted from The Lancet, Vol. number, Author(s), Title of article, Pages No., Copyright (Year), with permission from Elsevier."

4. Reproduction of this material is confined to the purpose and/or media for which permission is hereby given.

5. Altering/Modifying Material: Not Permitted. However figures and illustrations may be altered/adapted minimally to serve your work. Any other abbreviations, additions, deletions and/or any other alterations shall be made only with prior written authorization of Elsevier Ltd. (Please contact Elsevier at permissions@elsevier.com)

6. If the permission fee for the requested use of our material is waived in this instance, please be advised that your future requests for Elsevier materials may attract a fee. 
7. Reservation of Rights: Publisher reserves all rights not specifically granted in the combination of (i) the license details provided by you and accepted in the course of this licensing transaction, (ii) these terms and conditions and (iii) CCC's Billing and Payment terms and conditions.

8. License Contingent Upon Payment: While you may exercise the rights licensed immediately upon issuance of the license at the end of the licensing process for the transaction, provided that you have disclosed complete and accurate details of your proposed use, no license is finally effective unless and until full payment is received from you (either by publisher or by CCC) as provided in CCC's Billing and Payment terms and conditions. If full payment is not received on a timely basis, then any license preliminarily granted shall be deemed automatically revoked and shall be void as if never granted. Further, in the event that you breach any of these terms and conditions or any of CCC's Billing and Payment terms and conditions, the license is automatically revoked and shall be void as if never granted. Use of materials as described in a revoked license, as well as any use of the materials beyond the scope of an unrevoked license, may constitute copyright infringement and publisher reserves the right to take any and all action to protect its copyright in the materials.

9. Warranties: Publisher makes no representations or warranties with respect to the licensed material.

10. Indemnity: You hereby indemnify and agree to hold harmless publisher and CCC, and their respective officers, directors, employees and agents, from and against any and all claims arising out of your use of the licensed material other than as specifically authorized pursuant to this license.

11. No Transfer of License: This license is personal to you and may not be sublicensed, assigned, or transferred by you to any other person without publisher's written permission.

12. No Amendment Except in Writing: This license may not be amended except in a writing signed by both parties (or, in the case of publisher, by CCC on publisher's behalf).

13. Objection to Contrary Terms: Publisher hereby objects to any terms contained in any purchase order, acknowledgment, check endorsement or other writing prepared by you, which terms are inconsistent with these terms and conditions or CCC's Billing and Payment terms and conditions. These terms and conditions, together with CCC's Billing and Payment terms and conditions (which are incorporated herein), comprise the entire agreement between you and publisher (and CCC) concerning this licensing transaction. In the event of any conflict between your obligations established by these terms and conditions and those established by CCC's Billing and Payment terms and conditions, these terms and conditions shall control. 
14. Revocation: Elsevier or Copyright Clearance Center may deny the permissions described in this License at their sole discretion, for any reason or no reason, with a full refund payable to you. Notice of such denial will be made using the contact information provided by you. Failure to receive such notice will not alter or invalidate the denial. In no event will Elsevier or Copyright Clearance Center be responsible or liable for any costs, expenses or damage incurred by you as a result of a denial of your permission request, other than a refund of the amount(s) paid by you to Elsevier and/or Copyright Clearance Center for denied permissions.

\section{LIMITED LICENSE}

The following terms and conditions apply to specific license types:

15. Translation: This permission is granted for non-exclusive world English rights only unless your license was granted for translation rights. If you licensed translation rights you may only translate this content into the languages you requested. A professional translator must perform all translations and reproduce the content word for word preserving the integrity of the article. If this license is to re-use 1 or 2 figures then permission is granted for non-exclusive world rights in all languages.

16. Website: The following terms and conditions apply to electronic reserve and author websites:

Electronic reserve: If licensed material is to be posted to website, the web site is to be password-protected and made available only to bona fide students registered on a relevant course if:

This license was made in connection with a course,

This permission is granted for 1 year only. You may obtain a license for future website posting, All content posted to the web site must maintain the copyright information line on the bottom of each image,

A hyper-text must be included to the Homepage of the journal from which you are licensing at http://www.sciencedirect.com/science/journal/xxxxx or the Elsevier homepage for books at http://www.elsevier.com , and

Central Storage: This license does not include permission for a scanned version of the material to be stored in a central repository such as that provided by Heron/XanEdu.

17. Author website for journals with the following additional clauses:

This permission is granted for 1 year only. You may obtain a license for future website posting,

All content posted to the web site must maintain the copyright information line on the bottom of each image, and

The permission granted is limited to the personal version of your paper. You are not allowed to download and post the published electronic version of your article (whether PDF or HTML, proof or final version), nor may you scan the printed edition to create an electronic version, 
A hyper-text must be included to the Homepage of the journal from which you are licensing at http://www.sciencedirect.com/science/journal/xxxxx , or the Elsevier homepage for books at http://www.elsevier.com and

Central Storage: This license does not include permission for a scanned version of the material to be stored in a central repository such as that provided by Heron/XanEdu.

18. Author website for books with the following additional clauses:

Authors are permitted to place a brief summary of their work online only. A hyper-text must be included to the Elsevier homepage at http://www.elsevier.com This permission is granted for 1 year only. You may obtain a license for future website posting,

All content posted to the web site must maintain the copyright information line on the bottom of each image, and

The permission granted is limited to the personal version of your paper. You are not allowed to download and post the published electronic version of your article (whether PDF or HTML, proof or final version), nor may you scan the printed edition to create an electronic version, A hyper-text must be included to the Homepage of the journal from which you are licensing at http://www.sciencedirect.com/science/journal/xxxxx , or the Elsevier homepage for books at http://www.elsevier.com and Central Storage: This license does not include permission for a scanned version of the material to be stored in a central repository such as that provided by Heron/XanEdu.

19. Website (regular and for author): "A hyper-text must be included to the Homepage of the journal from which you are licensing at http://www.sciencedirect.com/science/journal/xxxxx."

20. Thesis/Dissertation: If your license is for use in a thesis/dissertation your thesis may be submitted to your institution in either print or electronic form. Should your thesis be published commercially, please reapply for permission. These requirements include permission for the Library and Archives of Canada to supply single copies, on demand, of the complete thesis and include permission for UMI to supply single copies, on demand, of the complete thesis. Should your thesis be published commercially, please reapply for permission.

\section{A.6: PERMISSIONS FOR FiguRE 19 AND FIGURE 116.}

This is a License Agreement between Mark H Griep ("You") and Nature Publishing Group ("Nature Publishing Group"). The license consists of your order details, the terms and conditions provided by Nature Publishing Group, and the payment terms 
and conditions.

Get the printable license.

License Number $\quad 2063270626297$

License date Nov 06, 2008

Licensed content $\quad$ Nature Publishing Group
publisher

Licensed content

publication

Nature Materials

Licensed content title

Self-assembled nanoscale biosensors based on quantum dot FRET donors

Licensed content author

Igor L. Medintz, Aaron R. Clapp, Hedi Mattoussi, Ellen R. Goldman, Brent Fisher, J. Matthew Mauro

Volume number

Issue number

Pages

Year of publication 2003

Portion used Figures / tables

Requestor type Student

Type of Use Thesis / Dissertation

PO Number

Total $\quad 0.00$ USD

This is a License Agreement between Mark H Griep ("You") and Nature Publishing Group ("Nature Publishing Group"). The license consists of your order details, the terms and conditions provided by Nature Publishing Group, and the payment terms and conditions.

License Number

2063270626297

License date

Nov 06, 2008

Licensed content publisher

Nature Publishing Group 
Licensed content publication

Nature Materials

Licensed content title

Self-assembled nanoscale biosensors based on quantum dot FRETdonors

Licensed content author

Igor L. Medintz ,Aaron R. Clapp ,Hedi Mattoussi ,Ellen R. Goldman ,Brent Fisher et al.

Volume number

Issue number

Pages

Year of publication

2003

Portion used

Figures / tables

Requestor type

Student

Type of Use

Thesis / Dissertation

PO Number

Total

0.00 USD

Terms and Conditions

Terms and Conditions for Permissions 
Nature Publishing Group hereby grants you a non-exclusive license to reproduce this material for this purpose, and for no other use, subject to the conditions below:

1. NPG warrants that it has, to the best of its knowledge, the rights to license reuse of this material. However, you should ensure that the material you are requesting is original to Nature Publishing Group and does not carry the copyright of another entity (as credited in the published version). If the credit line on any part of the material you have requested indicates that it was reprinted or adapted by NPG with permission from another source, then you should also seek permission from that source to reuse the material.

2. Permission granted free of charge for material in print is also usually granted for any electronic version of that work, provided that the material is incidental to the work as a whole and that the electronic version is essentially equivalent to, or substitutes for, the print version. Where print permission has been granted for a fee, separate permission must be obtained for any additional, electronic re-use (unless, as in the case of a full paper, this has already been accounted for during your initial request in the calculation of a print run). NB: In all cases, web-based use of full-text articles must be authorized separately through the 'Use on a Web Site' option when requesting permission.

3. Permission granted for a first edition does not apply to second and subsequent editions and for editions in other languages (except for signatories to the STM Permissions Guidelines, or where the first edition permission was granted for free).

4. Nature Publishing Group's permission must be acknowledged next to the figure, table or abstract in print. In electronic form, this acknowledgement must be visible at the same time as the figure/table/abstract, and must be hyperlinked to the journal's homepage.

5. The credit line should read:

Reprinted by permission from Macmillan Publishers Ltd: [JOURNAL NAME] (reference citation), copyright (year of publication)

For AOP papers, the credit line should read:

Reprinted by permission from Macmillan Publishers Ltd: [JOURNAL NAME], advance online publication, day month year (doi: 10.1038/sj.[JOURNAL ACRONYM].XXXXX)

6. Adaptations of single figures do not require NPG approval. However, the adaptation should be credited as follows:

Adapted by permission from Macmillan Publishers Ltd: [JOURNAL NAME] 
(reference citation), copyright (year of publication)

7. Translations of 401 words up to a whole article require NPG approval. Please visit http://www.macmillanmedicalcommunications.com for more information. Translations of up to a 400 words do not require NPG approval. The translation should be credited as follows:

Translated by permission from Macmillan Publishers Ltd: [JOURNAL NAME] (reference citation), copyright (year of publication).

We are certain that all parties will benefit from this agreement and wish you the best in the use of this material. Thank you.

\title{
A.7: PeRMissions fOR Figure 20 AND Figure 21.
}

For your records the following options have been submitted.

\author{
Name : Mark Griep \\ Address : 815 R.L. Smith Houghton, MI 49931 \\ Tel : 906-281-2939 \\ Fax : 906-487-2822 \\ Email : mhgriep@mtu.edu
}

I am preparing work for publication:

Article/Chapter title :

Journal/Book Title : Integration of quantum dots with the optical protein

Editor/Author(s) : Mark Griep

Publisher : Michigan Technological University

I would very much appreciate your permission to use the following material:

Journal/Book Title : Chem. Commun.

Editor/Author(s) : Dyadyusha et. al.

Volume Number :

Year of Publication : 2005 
Description of Material : Figure 5 graphic

Page(s) : 3202

Journal/Book Title : Chem. Commun.

Editor/Author(s) : Dyadyusha et. al.

Volume Number :

Year of Publication : 2005

Description of Material : Figure 6 graphic

Page(s) : 3203 This material would be incorporated into the introductory
chapter of my PhD Dissertation.

\section{Dear Mark Griep}

The Royal Society of Chemistry hereby grants permission for the use of the material specified below in the work described and in all subsequent editions of the work for distribution throughout the world, in all media including electronic and microfilm. You may use the material in conjunction with computer-based electronic and information retrieval systems, grant permissions for photocopying, reproductions and reprints, translate the material and to publish the translation, and authorize document delivery and abstracting and indexing services. The Royal Society of Chemistry is a signatory to the STM Guidelines on Permissions (available on request).

Please note that if the material specified below or any part of it appears with credit or acknowledgement to a third party then you must also secure permission from that third party before reproducing that material.

Please ensure that the published article carries a credit to The Royal Society of Chemistry in the following format:

[Original citation] - Reproduced by permission of The Royal Society of Chemistry

and that any electronic version of the work includes a hyperlink to the article on the Royal Society of Chemistry website. The recommended form for the hyperlink is http://dx.doi.org/10.1039/DOI suffix, for example in the link http://dx.doi.org/10.1039/b110420a the DOI suffix is 'b110420a'. To find the relevant DOI suffix for the RSC paper in question, go to the Journals section of the website and locate your paper in the list of papers for the volume and issue of your specific journal. You will find the DOI suffix quoted there.

Regards

Gill Cockhead

Contracts \& Copyright Executive

Gill Cockhead (Mrs), Contracts \& Copyright Executive 
Royal Society of Chemistry, Thomas Graham House

Science Park, Milton Road, Cambridge CB4 0WF, UK

Tel +44 (0) 1223432134, Fax +44 (0) 1223423623

http://www.rsc.org

\title{
A.8: Permissions for Figure 82.
}

Hi, Mark:

You have our permission. If possible, please credit Thermo Fisher Scientific somewhere. That's our company name.

If you want to reference the specific product, it's Thermo Scientific Pierce EDC. (The word Fisher is in the company name, but not in the product branding.)

If you have questions, please let me know.

Thanks for your support of our products and good luck with your dissertation!

Best wishes,

Holly

Holly Schubert

Associate Director, Marketing Communications

Thermo Scientific Pierce Protein Research

Thermo Fisher Scientific

3747 N Meridian Road

PO Box 117

Rockford, IL 61105

Tel: 815-968-0747, ext. 339

Email: holly.schubert@thermofisher.com

www.thermo.com/pierce

----- Forwarded by Greg Hermanson/UsRfd/Perbio on 10/28/2008 08:53 AM -----

\author{
Mark Griep <mhgriep@mtu.edu $>$ To: \\ greg.hermanson@piercenet.com \\ 10/27/2008cc: \\ 04:54 PM Subject: Use of image on Pierce \\ website
}

$\mathrm{Hi}$, 
I've been using your Pierce EDC cross-linker for my $\mathrm{PhD}$ work and am now writing up my dissertation. I would like to use your schematic of the EDC mechanism, which is the 2nd graph down on the following website:

http://www.piercenet.com/Objects/View.cfm?type=ProductFamily\&ID=02030312

I am writing to ask for permission to use this image in my dissertation for explanatory purposes. Please let me know if this is possible or if there is someone else I should contact. Thank you very much and it's been a pleasure doing business with you.

-Mark Griep

906-281-2939 